Supporting Information for:

\title{
DFT STUDY ON THE PALLADIUM CATALYSED ALLYLATION OF PRIMARY AMINES BY ALLYLIC ALCOHOL
}

\author{
Olivier Piechaczyk, Claire Thoumazet, Yves Jean and Pascal le Floch*. \\ Laboratoire Hétéroéléments et Coordination, UMR CNRS 7653, Ecole Polytechnique, \\ 91128 Palaiseau cedex, France.
}

Experimental Data: 3

DFT Data: 5

I. Molecules.

Optimized geometry, three lower frequencies, thermochemistry and PCM energy for water. ............................. 5

Optimized geometry, three lower frequencies, thermochemistry and PCM energy for allylalcohol...................... 5

Optimized geometry, three lower frequencies, thermochemistry and PCM energy for methylamine................... 6

Optimized geometry, three lower frequencies, thermochemistry and PCM energy for methylammonium.......... 7

Optimized geometry, three lower frequencies, thermochemistry and PCM energy for allylmethylamine............ 8

Optimized geometry, three lower frequencies, thermochemistry and PCM energy for allylmethylammonium... 9

Optimized geometry, three lower frequencies, thermochemistry and PCM energy for aniline............................ 10

Optimized geometry, three lower frequencies, thermochemistry and PCM energy for phenylammonium........ 11

Optimized geometry, three lower frequencies, thermochemistry and PCM energy for allylphenylammonium. 13

Optimized geometry, three lower frequencies, thermochemistry and PCM energy for triflate anion................. 14

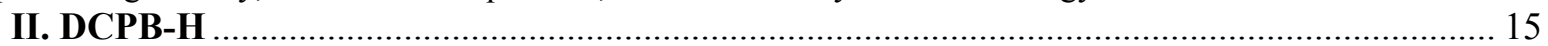

Optimized geometry, three lower frequencies, thermochemistry and PCM energy for 1a. .................................... 15

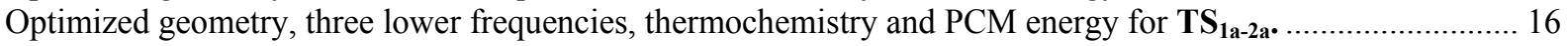

Optimized geometry, three lower frequencies, thermochemistry and PCM energy for 2a................................. 18

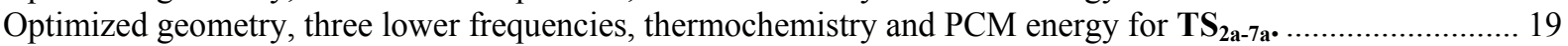

Optimized geometry, three lower frequencies, thermochemistry and PCM energy for 3a................................ 21

Optimized geometry, three lower frequencies, thermochemistry and PCM energy for 3'a.................................. 22

Optimized geometry, three lower frequencies, thermochemistry and PCM energy for 4a..................................2 23

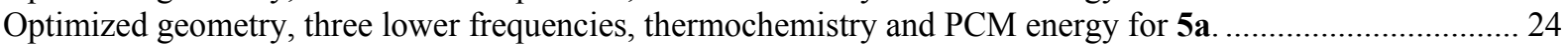

Optimized geometry, three lower frequencies, thermochemistry and PCM energy for $\mathbf{5}$ 'a.................................2 26

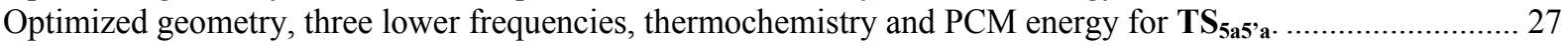

Optimized geometry, three lower frequencies, thermochemistry and PCM energy for 6a.................................2 29

Optimized geometry, three lower frequencies, thermochemistry and PCM energy for 7a................................ 30

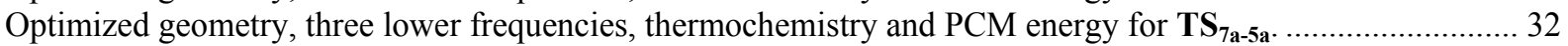

Optimized geometry, three lower frequencies, thermochemistry and PCM energy for 8a. .................................. 33

Optimized geometry, three lower frequencies, thermochemistry and PCM energy for 9a....................................35

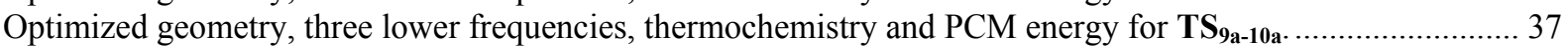

Optimized geometry, three lower frequencies, thermochemistry and PCM energy for 10a................................. 38

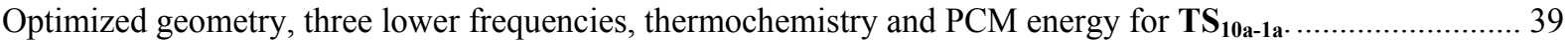

Optimized geometry, three lower frequencies, thermochemistry and PCM energy for 11a................................ 40

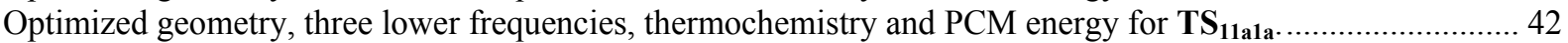

Optimized geometry, three lower frequencies, thermochemistry and PCM energy for 12a................................. 44

Optimized geometry, three lower frequencies, thermochemistry and PCM energy for 13a................................. 45

Optimized geometry, three lower frequencies, thermochemistry and PCM energy for 14a................................. 46

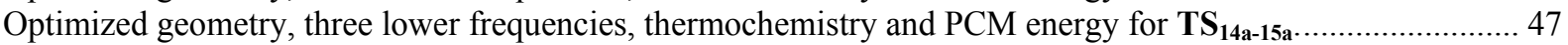

Optimized geometry, three lower frequencies, thermochemistry and PCM energy for 15a................................. 49

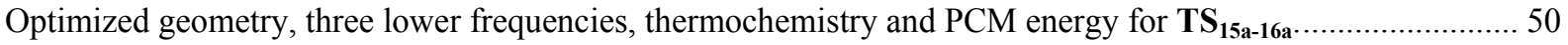

Optimized geometry, three lower frequencies, thermochemistry and PCM energy for 16a................................. 52

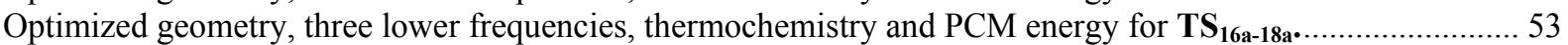

Optimized geometry, three lower frequencies, thermochemistry and PCM energy for 17a................................ 55

Optimized geometry, three lower frequencies, thermochemistry and PCM energy for 18a............................... 56

Optimized geometry, three lower frequencies, thermochemistry and PCM energy for 19a................................58

Optimized geometry, three lower frequencies, thermochemistry and PCM energy for $\mathbf{T S}_{\mathbf{1 a}-2 \mathbf{2} \mathbf{a}} \ldots \ldots \ldots \ldots \ldots \ldots \ldots \ldots \ldots . . . . . . . . . . . .59$

Optimized geometry, three lower frequencies, thermochemistry and PCM energy for 20a................................... 60

Optimized geometry, three lower frequencies, thermochemistry and PCM energy for 21a................................. 62 
Optimized geometry, three lower frequencies, thermochemistry and PCM energy for $\mathbf{T S}_{\mathbf{2 1} \mathbf{a}-\mathbf{1} \mathbf{a}, \ldots \ldots \ldots \ldots \ldots \ldots \ldots \ldots . . . \ldots} 65$

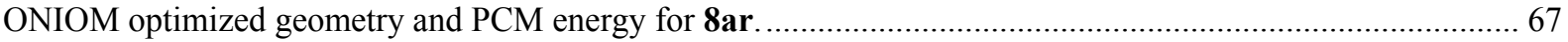

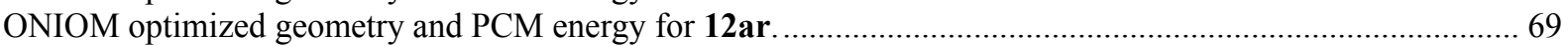

III. DAD-H

Optimized geometry, three lower frequencies, thermochemistry and PCM energy for 1b............................. 72

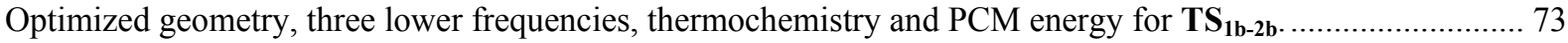

Optimized geometry, three lower frequencies, thermochemistry and PCM energy for $\mathbf{2 b}$............................... 74

Optimized geometry, three lower frequencies, thermochemistry and PCM energy for 11b............................. 76

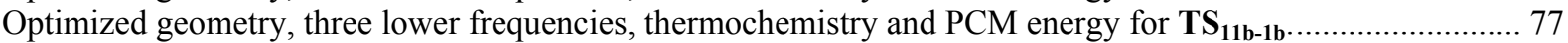

Optimized geometry, three lower frequencies, thermochemistry and PCM energy for 12b.......................... 79

Optimized geometry, three lower frequencies, thermochemistry and PCM energy for 13b............................ 80

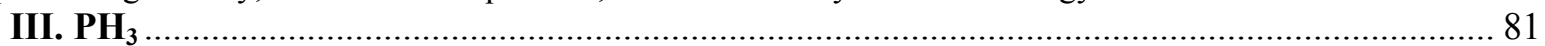

Optimized geometry, three lower frequencies and PCM energy for 1c. ................................................. 81

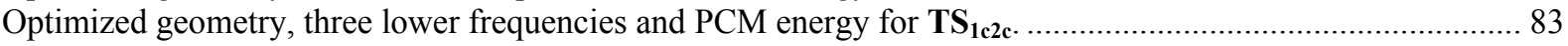

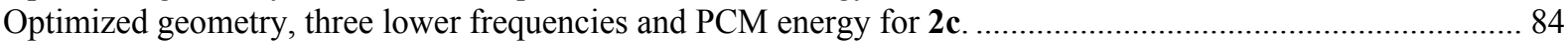

Optimized geometry, three lower frequencies and PCM energy for 11c. ................................................ 85

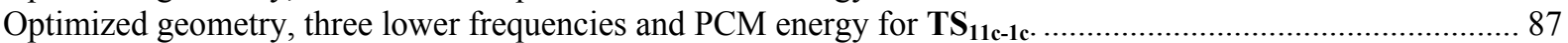

Optimized geometry, three lower frequencies and PCM energy for 12c. ............................................... 89

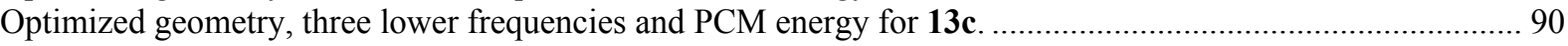

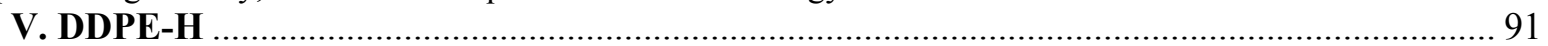

Optimized geometry, three lower frequencies, thermochemistry and PCM energy for 1d.............................91

Optimized geometry, three lower frequencies, thermochemistry and PCM energy for TS $\mathbf{T S}_{\mathbf{1 d}-\mathbf{2 d}} \ldots \ldots \ldots \ldots \ldots \ldots \ldots \ldots . . . . . . . . . . . .92$

Optimized geometry, three lower frequencies, thermochemistry and PCM energy for 2d.............................94

Optimized geometry, three lower frequencies, thermochemistry and PCM energy for 11d............................95

Optimized geometry, three lower frequencies, thermochemistry and PCM energy for $\mathbf{T S}_{\mathbf{1 1 d}-\mathbf{1 d} \ldots \ldots \ldots \ldots \ldots \ldots \ldots . . . . . . . . . . . . .19}$

Optimized geometry, three lower frequencies, thermochemistry and PCM energy for 12d........................... 99

Optimized geometry, three lower frequencies, thermochemistry and PCM energy for 13d..........................99

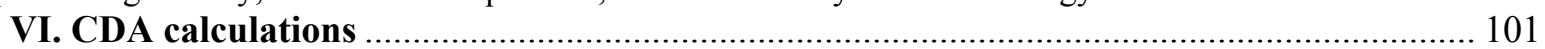

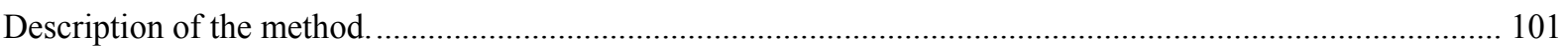

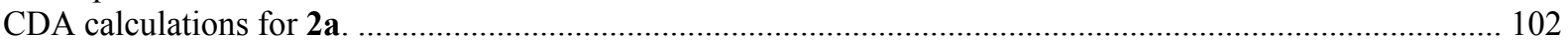

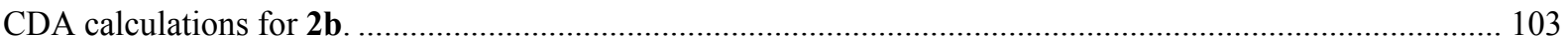

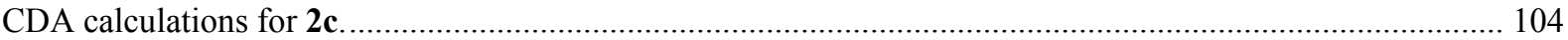

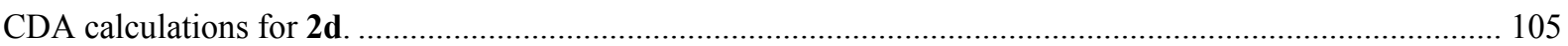

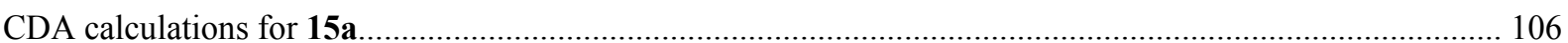

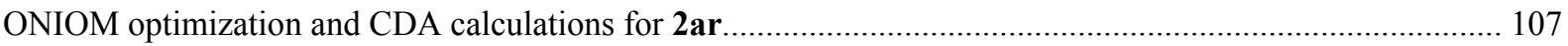

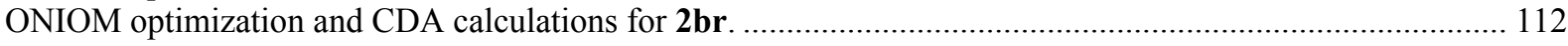

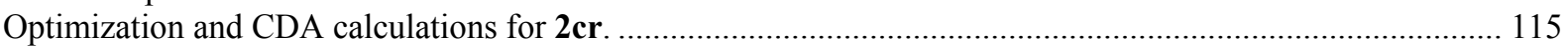

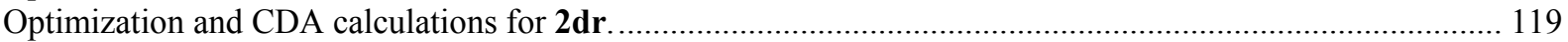

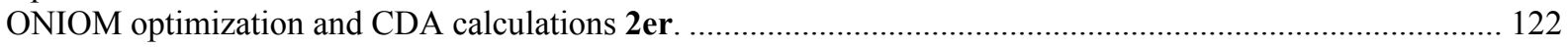




\section{Experimental Data:}

\section{Synthesis of complex [Bistriphenylphosphite $\left.\operatorname{Pd}\left(\eta_{3}-\mathrm{C}_{3} \mathrm{H}_{5}\right)^{+}\right][\mathrm{OTf}]$ (1e):}

To a solution of $[\mathrm{Pd}(\mathrm{allyl}) \mathrm{Cl}]_{2}(0.25 \mathrm{eq}, 59.5 \mathrm{mg})$ in dichloromethane $(2 \mathrm{~mL})$ was added triphenylphosphite $(200 \mathrm{mg}, 0.64 \mathrm{mmol})$. After stirring $5 \mathrm{mn}$, the formation the bisphosphite complex is checked by ${ }^{31} \mathrm{P}-\mathrm{NMR}$ (124.3 ppm). Then the chloride was abstracted with a silver salt $\mathrm{AgOTf}$ (83mg). Filtration of $\mathrm{AgCl}$ on celite followed by evaporation of the dichloromethane gives the complexes as powders in very good yields $(97 \%, 285 \mathrm{mg})$.

${ }^{31} \mathrm{P}$ NMR $\left(\mathrm{CD}_{2} \mathrm{Cl}_{2}, 25^{\circ} \mathrm{C}\right): \delta 120.0 ;{ }^{1} \mathrm{H} \mathrm{NMR}\left(\mathrm{CD}_{2} \mathrm{Cl}_{2}, 25^{\circ} \mathrm{C}\right): \delta 2.34$ (AA'BB'CXX', $\Sigma J=$ $33.1 \mathrm{~Hz}, 2 \mathrm{H}$, Hallyl AA' or BB'), 4.07 (AA'BB'CXX', $\Sigma J=18.1 \mathrm{~Hz}, 2 \mathrm{H}$, Hallyl BB' or AA'), $4.77\left(\mathrm{AB}_{2} \mathrm{C}_{2} \mathrm{X}_{2}, \Sigma J=41.6 \mathrm{~Hz}, 1 \mathrm{H}\right.$, Hallyl), 7.04 (m, $\left.\Sigma J=8.0 \mathrm{~Hz}, 12 \mathrm{H}, \mathrm{Ph}\right), 7.30$ (m, $\Sigma$

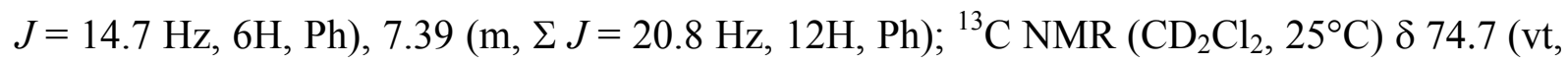
AXX', $\Sigma J=44.2 \mathrm{~Hz}, \mathrm{CH}_{2 \text { allyl }}$ ), 121.0 (vt, AXX', $\Sigma J=5.7 \mathrm{~Hz}, \mathrm{Ph}$ ), 124.6 (vt, AXX', $\Sigma J=$ $20.8 \mathrm{~Hz}, \mathrm{CH}_{\text {allyl }}$ ), 127.1 (s, Ph), 131.1 (s, Ph), 150.3 (vt, AXX', $\Sigma J=5.5 \mathrm{~Hz}$, ipso-Ph). Anal. Calcd for $\mathrm{C}_{40} \mathrm{H}_{35} \mathrm{~F}_{3} \mathrm{O}_{9} \mathrm{P}_{2} \mathrm{PdS}$ : C, 52.38; H, 3.85; Found: C, 52.17; H, 3.84 .

\section{Synthesis of complex [1,2-bis(diphenylphosphino)ethane $\left.\operatorname{Pd}\left(\eta_{3}-\mathrm{C}_{3} \mathrm{H}_{5}\right)^{+}\right][\mathrm{OTf}]$ (1d):}

To a solution of 1,2-bis(diphenylphosphino)ethane $(100 \mathrm{mg}, 0.25 \mathrm{mmol})$ in dichloromethane $(2 \mathrm{~mL})$ was added $[\mathrm{Pd}(\text { allyl }) \mathrm{Cl}]_{2}(0.13 \mathrm{mmol}, 45.9 \mathrm{mg})$. After $5 \mathrm{mn}$ stirring, the formation of a bidentate complex was checked by ${ }^{31} \mathrm{P}-\mathrm{NMR}$ (51.2 ppm). Then the silver salt AgOTf was added $(0.25 \mathrm{mmol}, 64.5 \mathrm{mg})$. Filtration of $\mathrm{AgCl}$ on celite followed by evaporation of the dichloromethane yielded the complexes as white powders in very good yields $(98 \%, 170 \mathrm{mg})$. ${ }^{31} \mathrm{P}$ NMR $\left(\mathrm{CDCl}_{3}, 25^{\circ} \mathrm{C}\right): \delta 51.4 ;{ }^{1} \mathrm{H} \mathrm{NMR}\left(\mathrm{CDCl}_{3}, 25^{\circ} \mathrm{C}\right): \delta 2.70\left(\mathrm{~m}, \mathrm{~A}_{2} \mathrm{~A}^{\prime}{ }_{2} \mathrm{XX}, \Sigma J=99.5\right.$ $\mathrm{Hz}, 4 \mathrm{H}, \mathrm{CH}_{2} \mathrm{PPh}_{2}$ ), 3.39 (vddd, AA'BB'CXX', $\Sigma J=24.8 \mathrm{~Hz}, 2 \mathrm{H}, \mathrm{CH}_{\text {allyl }}$ ), 4.86 (m, AA'BB'CXX', $\Sigma J=12.4 \mathrm{~Hz}, 2 \mathrm{H}, \mathrm{CH}_{\text {allyl }}$ ), 5.81 (vtt, $\mathrm{AB}_{2} \mathrm{C}_{2} \mathrm{X}_{2}, \Sigma J=42.5 \mathrm{~Hz}, 1 \mathrm{H}, \mathrm{CH}_{\text {allyl }}$ ), 7.43-7.60 (m, 20H, Ph); ${ }^{13} \mathrm{C} \mathrm{NMR}\left(\mathrm{CDCl}_{3}, 25^{\circ} \mathrm{C}\right) \delta 27.5$ (vt, AXX', $\Sigma J=46.0 \mathrm{~Hz}, \mathrm{CH}_{2} \mathrm{PPh}$ ), 71.4 (vt, $\mathrm{AXX}, \Sigma J=33.2 \mathrm{~Hz}, \mathrm{CH}_{2 \mathrm{allyl}}$ ), 123.6 (vt, AXX', $\Sigma J=12.2 \mathrm{~Hz}, \mathrm{CH}_{\text {allyl }}$ ), 129.9132.8 (Ph). Anal. Calcd for $\mathrm{C}_{31} \mathrm{H}_{29} \mathrm{~F}_{6} \mathrm{NO}_{4} \mathrm{P}_{2} \mathrm{PdS}_{2}$ : C, 45.07; H, 3.54; Found: C, 44.85; H, 3.53 .

\section{Synthesis of complex [glyoxal-bis-(2,6-diisopropylphenyl)imine $\left.\operatorname{Pd}\left(\eta_{3}-\mathrm{C}_{3} \mathrm{H}_{5}\right)^{+}\right][\mathrm{OTf}]$} (1b):

To a solution of glyoxal-bis-(2,6-diisopropylphenyl)imine (100mg, 0.26mmol) in dichloromethane $(2 \mathrm{~mL})$ was added $[\mathrm{Pd}(\text { allyl }) \mathrm{Cl}]_{2}(48.6 \mathrm{mg}, 0.13 \mathrm{mmol})$. After $5 \mathrm{mn}$ stirring, 
the silver salt AgOTf $(0.26 \mathrm{mmol}, 68.2 \mathrm{mg})$ was added and the pale yellow solution turned orange. Filtration of $\mathrm{AgCl}$ on celite followed by evaporation of the dichloromethane yielded the complexes as orange powders in very good yields $(97 \%, 170 \mathrm{mg})$.

${ }^{1} \mathrm{H} \operatorname{NMR}\left(\mathrm{CDCl}_{3}, 25^{\circ} \mathrm{C}\right): \delta 1.32\left(\mathrm{~m}, 24 \mathrm{H}, \mathrm{CHCH}_{3}\right), 3.03\left(\mathrm{~m}, 2 \mathrm{H}, \mathrm{CHCH}_{3}\right), 3.17(\mathrm{vd}$, AA'BB'C, $\left.\Sigma J=12.8 \mathrm{~Hz}, 2 \mathrm{H}, \mathrm{CH}_{\text {allyl }}\right), 3.25\left(\mathrm{~m}, 2 \mathrm{H}, \mathrm{CHCH}_{3}\right), 3.59$ (vd, AA'BB'C, $\Sigma J=7.0$ $\left.\mathrm{Hz}, 2 \mathrm{H}, \mathrm{CH}_{\text {allyl }}\right), 5.56$ (tt, $J=7.0$ and $\left.12.8 \mathrm{~Hz}, 1 \mathrm{H}, \mathrm{CH}_{\text {allyl }}\right), 7.26-7.40$ (m, 6H, $\left.m-\mathrm{Ph}\right), 8.61$ (s, $2 \mathrm{H}, \mathrm{CH}=\mathrm{N}) ;{ }^{13} \mathrm{C} \mathrm{NMR}\left(\mathrm{CDCl}_{3}, 25^{\circ} \mathrm{C}\right) \delta 23.0\left(\mathrm{CHCH}_{3}\right), 23.7(\mathrm{CH}=\mathrm{N}), 28.9\left(\mathrm{CHCH}_{3}\right), 65.1$ $\left(\mathrm{CH}_{2 \text { allyl }}\right), 120.1\left(\mathrm{CH}_{\text {allyl }}\right), 124.1-128.8$ (m-Ph), 138.1 (o-Ph), 146.1 (ipso-Ph), 170.0 (p-Ph). Anal. Calcd for $\mathrm{C}_{30} \mathrm{H}_{41} \mathrm{~F}_{3} \mathrm{~N}_{2} \mathrm{O}_{3} \mathrm{PdS}$ : C, 53.53; H, 6.14; Found: C, 53.28; H, 6.12.

\section{Synthesis of complex [Bistriphenylphosphine $\left.\operatorname{Pd}(3)\left(\eta_{3}-\mathrm{C}_{3} \mathrm{H}_{5}\right)^{+}\right]\left[\mathrm{OTf}^{-}\right](1 \mathrm{c})$ :}

Amatore, C.; Jutand, A.; M'Barki, M. A.; Meyer, G.; Mottier, L. Eur. J. Inorg. Chem. 2001, $873-880$.

\section{General procedure for catalytic reactions:}

The catalyst $(1 \% \mathrm{~mol})$ was put in a schlenck tube with $\mathrm{MgSO}_{4}(0.25 \mathrm{~g})$ and THF $(1 \mathrm{~mL})$. Then the schlenck tube was filled with the allyl alcohol $(68 \mu \mathrm{L}, 1 \mathrm{mmol})$ and the aniline $(182 \mu \mathrm{L}, 2$ mmol). The mixture was stirred at room temperature. The progress of the reaction was monitored by GC. The mixture was filtered to get rid of the $\mathrm{MgSO}_{4}$, and the solvent evaporated under vacuo. The product was then isolated by column chromatography on alumina (hexanes).

\section{$N$-(2-propenyl)aniline}

F. Ozawa, H. Okamoto, S. kawagishi, S. Yamamoto, T. Minami, M; Yoshifuji, J. Am. Chem. Soc., 2002, 124, 10968. 


\section{DFT Data:}

Complete Reference 23: Gaussian 03 Revision C.02, M. J. Frisch, G. W. Trucks, H. B. Schlegel, G. E. Scuseria, M. A. Robb, J. R. Cheeseman, J. A. Montgomery, Jr., T. Vreven, K. N. Kudin, J. C. Burant, J. M. Millam, S. S. Iyengar, J. Tomasi, V. Barone, B. Mennucci, M. Cossi, G. Scalmani, N. Rega, G. A. Petersson, H. Nakatsuji, M. Hada, M. Ehara, K. Toyota, R. Fukuda, J. Hasegawa, M. Ishida, T. Nakajima, Y. Honda, O. Kitao, H. Nakai, M. Klene, X. Li, J. E. Knox, H. P. Hratchian, J. B. Cross, V. Bakken, C. Adamo, J. Jaramillo, R. Gomperts, R. E. Stratmann, O. Yazyev, A. J. Austin, R. Cammi, C. Pomelli, J. W. Ochterski, P. Y. Ayala, K. Morokuma, G. A. Voth, P. Salvador, J. J. Dannenberg, V. G. Zakrzewski, S. Dapprich, A. D. Daniels, M. C. Strain, O. Farkas, D. K. Malick, A. D. Rabuck, K. Raghavachari, J. B. Foresman, J. V. Ortiz, Q. Cui, A. G. Baboul, S. Clifford, J. Cioslowski, B. B. Stefanov, G. Liu, A. Liashenko, P. Piskorz, I. Komaromi, R. L. Martin, D. J. Fox, T. Keith, M. A. Al-Laham, C. Y. Peng, A. Nanayakkara, M. Challacombe, P. M. W. Gill, B. Johnson, W. Chen, M. W. Wong, C. Gonzalez, and J. A. Pople, Gaussian, Inc., Wallingford CT.

\section{Molecules}

Optimized geometry, three lower frequencies, thermochemistry and PCM energy for water.

\begin{tabular}{|c|c|c|c|c|c|c|c|c|c|}
\hline Center & Atomic & & omic & \multicolumn{6}{|c|}{ Coordinates (Angstroms) } \\
\hline Number & Number & & ype & & $\mathrm{x}$ & Y & & Z & \\
\hline $\begin{array}{l}1 \\
2 \\
3\end{array}$ & $\begin{array}{l}8 \\
1 \\
1\end{array}$ & & $\begin{array}{l}0 \\
0 \\
0\end{array}$ & $\begin{array}{l}0 . \\
0 . \\
0 .\end{array}$ & $\begin{array}{l}00000 \\
00000 \\
00000\end{array}$ & $\begin{array}{r}0.000000 \\
0.768778 \\
-0.768778\end{array}$ & $\begin{array}{r}0 \\
-0 \\
-0\end{array}$ & $\begin{array}{l}117169 \\
468677 \\
468677\end{array}$ & \\
\hline & & $\begin{array}{l}1 \\
\text { A1 }\end{array}$ & & & $\begin{array}{l}2 \\
\mathrm{~A} 1\end{array}$ & & & $\begin{array}{l}3 \\
\text { B2 }\end{array}$ & \\
\hline Frequencies & $5--1$ & 1671.245 & & & 773.42 & & & 3900.943 & \\
\hline Red. masses & $5--$ & 1.083 & & & 1.04 & & & 1.082 & \\
\hline Frc consts & -- & 1.782 & & & 8.76 & & & 9.707 & \\
\hline IR Inten & -- & 98.100 & & & 7.61 & & & 55.980 & \\
\hline $\begin{array}{rr}\text { Atom } & \text { AN } \\
1 & 8\end{array}$ & $\begin{array}{c}x \\
0.00\end{array}$ & $\begin{array}{l}\mathrm{Y} \\
0.00\end{array}$ & $\begin{array}{c}Z \\
0.07\end{array}$ & $\begin{array}{c}x \\
0.00\end{array}$ & $\begin{array}{l}Y \\
0.00\end{array}$ & $\begin{array}{c}Z \\
0.05\end{array}$ & $\begin{array}{c}x \\
0.00\end{array}$ & $\begin{array}{c}Y \\
0.07\end{array}$ & $0^{Z} .00$ \\
\hline $\begin{array}{ll}1 & 8 \\
2 & 1\end{array}$ & $\begin{array}{l}0.00 \\
0.00\end{array}$ & $\begin{array}{r}0.00 \\
-0.43\end{array}$ & $\begin{array}{r}0.07 \\
-0.56\end{array}$ & $\begin{array}{l}0.00 \\
0.00\end{array}$ & $\begin{array}{l}0.00 \\
0.59\end{array}$ & $\begin{array}{r}0.05 \\
-0.39\end{array}$ & $\begin{array}{l}0.00 \\
0.00\end{array}$ & $\begin{array}{r}0.07 \\
-0.56\end{array}$ & $\begin{array}{l}0.00 \\
0.43\end{array}$ \\
\hline $3 \quad 1$ & 0.00 & 0.43 & -0.56 & 0.00 & -0.59 & -0.39 & 0.00 & -0.56 & -0.43 \\
\hline
\end{tabular}

thermodynamics:

Sum of electronic and zero-point Energies=

$-76.371716$

Sum of electronic and thermal Enthalpies=

Sum of electronic and thermal Free Energies=

$-76.367937$

$-76.389370$

Total free energy in solution:

with all non electrostatic terms

$($ a.u. $)=\quad-76.402193$

Optimized geometry, three lower frequencies, thermochemistry and PCM energy for allylalcohol. 


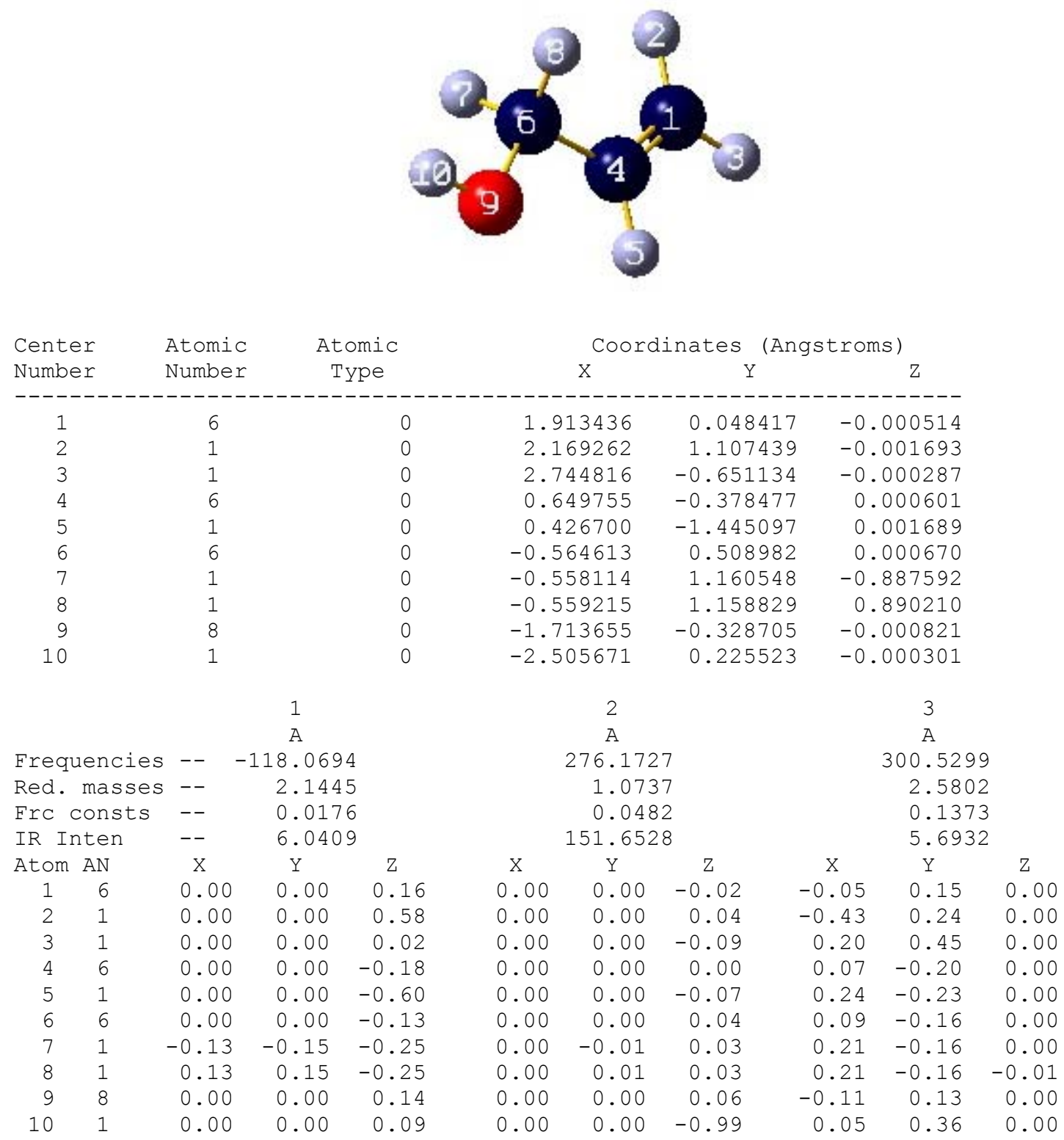

thermodynamics:

Sum of electronic and zero-point Energies=

$-192.959014$

Sum of electronic and thermal Enthalpies=

$-192.953686$

Sum of electronic and thermal Free Energies=

$-192.985436$

Total free energy in solution:

with all non electrostatic terms (a.u.) $=\quad-193.048039$

Optimized geometry, three lower frequencies, thermochemistry and PCM energy for methylamine.

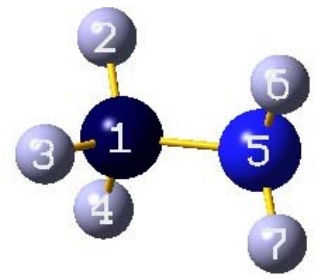




\begin{tabular}{|c|c|c|c|c|c|c|c|c|c|c|}
\hline \multirow{2}{*}{\multicolumn{2}{|c|}{$\begin{array}{l}\text { Center } \\
\text { Number }\end{array}$}} & \multirow{2}{*}{$\begin{array}{l}\text { Atomic } \\
\text { Number }\end{array}$} & \multirow{2}{*}{\multicolumn{2}{|c|}{$\begin{array}{c}\text { Atomic } \\
\text { Type }\end{array}$}} & \multicolumn{6}{|c|}{ Coordinates (Angstroms) } \\
\hline & & & & & & $\mathrm{x}$ & $Y$ & & Z & \\
\hline 1 & & 6 & & 0 & -0.7 & 05665 & 0.0000 & & 017872 & \\
\hline 2 & & 1 & & 0 & -1.1 & 12285 & 0.881 & -0 & 489106 & \\
\hline 3 & & 1 & & 0 & -1.0 & 81426 & -0.0000 & & 055425 & \\
\hline 4 & & 1 & & 0 & -1.1 & 12291 & -0.8815 & -0 & 489156 & \\
\hline 5 & & 7 & & 0 & 0.7 & 47646 & 0.000 & -0 & 120311 & \\
\hline 6 & & 1 & & 0 & 1.1 & 53235 & 0.816 & & 328891 & \\
\hline 7 & & 1 & & 0 & 1.1 & 53236 & -0.816 & & 328889 & \\
\hline & & & 1 & & & 2 & & & 3 & \\
\hline & & & A & & & A & & & A & \\
\hline Freq & lencies & $s--$ & 322.234 & & & 852.63 & & & 982.54 & \\
\hline Red. & masses & $S--$ & 1.029 & & & 1.20 & & & 1.05 & \\
\hline Frc & onsts & -- & 0.063 & & & 0.51 & & & $0.59^{\circ}$ & \\
\hline IR I & ten & -- & 45.898 & & & 197.03 & & & 0.14 & \\
\hline Atom & AN & X & $\mathrm{Y}$ & $\mathrm{z}$ & $\mathrm{x}$ & $\mathrm{Y}$ & Z & $\mathrm{x}$ & $\mathrm{Y}$ & z \\
\hline 1 & 6 & 0.00 & 0.00 & 0.00 & -0.03 & 0.00 & -0.01 & 0.00 & -0.06 & 0.00 \\
\hline 2 & 1 & -0.08 & -0.20 & -0.30 & -0.13 & 0.02 & 0.12 & 0.46 & 0.15 & -0.01 \\
\hline 3 & 1 & 0.00 & 0.31 & 0.00 & 0.25 & 0.00 & 0.08 & 0.00 & 0.27 & 0.00 \\
\hline 4 & 1 & 0.08 & -0.20 & 0.30 & -0.13 & -0.02 & 0.12 & -0.46 & 0.15 & 0.01 \\
\hline 5 & 7 & 0.00 & 0.04 & 0.00 & 0.08 & 0.00 & -0.09 & 0.00 & -0.01 & 0.00 \\
\hline 6 & 1 & 0.04 & -0.25 & 0.50 & -0.35 & -0.10 & 0.54 & -0.43 & 0.13 & 0.15 \\
\hline 7 & 1 & -0.04 & -0.25 & -0.50 & -0.35 & 0.10 & 0.54 & 0.43 & 0.13 & -0.15 \\
\hline
\end{tabular}

thermodynamics:

Sum of electronic and zero-point Energies= $\quad-95.760462$

Sum of electronic and thermal Enthalpies= $\quad-\mathbf{- 9 5 . 7 5 6 1 1 8}$

Sum of electronic and thermal Free Energies= $\quad-95.783363$

Total free energy in solution:

with all non electrostatic terms (a.u.) $=\quad-95.825742$

\section{Optimized geometry, three lower frequencies, thermochemistry and PCM energy for methylammonium.}

\begin{tabular}{|c|c|c|c|c|c|}
\hline \multirow{2}{*}{$\begin{array}{l}\text { Center } \\
\text { Number }\end{array}$} & \multirow{2}{*}{$\begin{array}{l}\text { Atomic } \\
\text { Number }\end{array}$} & \multirow{2}{*}{$\begin{array}{c}\text { Atomic } \\
\text { Type }\end{array}$} & \multicolumn{3}{|c|}{ Coordinates (Angstroms) } \\
\hline & & & $\mathrm{x}$ & Y & Z \\
\hline 1 & 6 & 0 & -0.798419 & 0.000000 & -0.000017 \\
\hline 2 & 1 & 0 & -1.142122 & 0.906115 & -0.499418 \\
\hline 3 & 1 & 0 & -1.141862 & -0.020461 & 1.034502 \\
\hline 4 & 1 & 0 & -1.142121 & -0.885660 & -0.534855 \\
\hline 5 & 7 & 0 & 0.708077 & 0.000000 & -0.000018 \\
\hline 6 & 1 & 0 & 1.086696 & 0.816788 & 0.493363 \\
\hline 7 & 1 & 0 & 1.086697 & -0.835647 & 0.460700 \\
\hline 8 & 1 & 0 & 1.086687 & 0.018859 & -0.954069 \\
\hline
\end{tabular}

\begin{tabular}{|c|c|c|c|c|c|c|}
\hline & & 1 & & 2 & & 3 \\
\hline & & A & & A & & A \\
\hline Frequencies & -- & 304.6560 & & 923.0601 & & 924.1880 \\
\hline Red. masses & -- & 1.0078 & & 1.0532 & & 1.0532 \\
\hline Frc consts & -- & 0.0551 & & 0.5287 & & 0.5300 \\
\hline IR Inten & -- & 0.0000 & & 26.5033 & & 26.5166 \\
\hline Atom AN & $\mathrm{X}$ & Y & Z & Y & Z & Y \\
\hline
\end{tabular}




$\begin{array}{rrrrrrrrrrr}1 & 6 & 0.00 & 0.00 & 0.00 & 0.00 & 0.00 & -0.03 & 0.00 & -0.03 & 0.00 \\ 2 & 1 & 0.00 & -0.19 & -0.34 & -0.26 & 0.02 & 0.18 & 0.39 & 0.15 & 0.05 \\ 3 & 1 & 0.00 & 0.39 & 0.01 & 0.47 & -0.02 & 0.12 & 0.04 & 0.21 & 0.01 \\ 4 & 1 & 0.00 & -0.20 & 0.33 & -0.20 & -0.05 & 0.19 & -0.42 & 0.14 & -0.02 \\ 5 & 7 & 0.00 & 0.00 & 0.00 & 0.00 & 0.00 & -0.05 & 0.00 & -0.05 & 0.00 \\ 6 & 1 & 0.00 & -0.22 & 0.36 & -0.24 & -0.05 & 0.22 & -0.50 & 0.18 & -0.01 \\ 7 & 1 & 0.00 & -0.20 & -0.37 & -0.31 & 0.01 & 0.22 & 0.45 & 0.19 & 0.05 \\ 8 & 1 & 0.00 & 0.42 & 0.01 & 0.55 & -0.02 & 0.17 & 0.04 & 0.24 & 0.02\end{array}$

thermodynamics:

Sum of electronic and zero-point Energies=

$-96.102343$

Sum of electronic and thermal Enthalpies=

$-96.097964$

Sum of electronic and thermal Free Energies=

$-96.125512$

Total free energy in solution:

with all non electrostatic terms

$-96.273834$

\section{Optimized geometry, three lower frequencies, thermochemistry and PCM energy for allylmethylamine.}

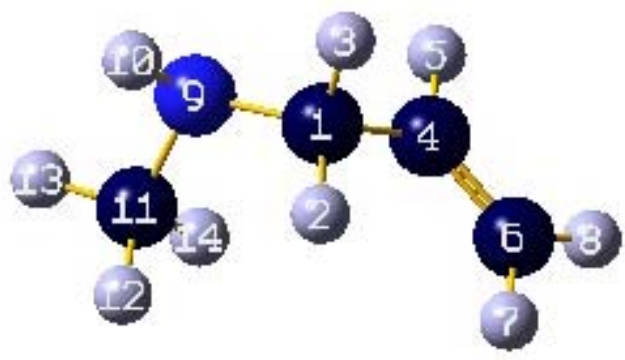

\begin{tabular}{|c|c|c|c|c|c|}
\hline Center & Atomic & Atomic & $\mathrm{COO}$ & inates (An & troms) \\
\hline Number & Number & Type & $\mathrm{X}$ & $\mathrm{Y}$ & Z \\
\hline 1 & 6 & 0 & 0.022464 & 0.841771 & 0.327100 \\
\hline 2 & 1 & 0 & 0.100683 & 0.585756 & 1.402307 \\
\hline 3 & 1 & 0 & 0.124434 & 1.933417 & 0.247327 \\
\hline 4 & 6 & 0 & 1.155478 & 0.197880 & -0.421408 \\
\hline 5 & 1 & 0 & 1.139386 & 0.348997 & -1.501814 \\
\hline 6 & 6 & 0 & 2.139252 & -0.508237 & 0.140484 \\
\hline 7 & 1 & 0 & 2.173584 & -0.685987 & 1.214688 \\
\hline 8 & 1 & 0 & 2.949152 & -0.930074 & -0.449912 \\
\hline 9 & 7 & 0 & -1.270210 & 0.488142 & -0.250122 \\
\hline 10 & 1 & 0 & -1.976989 & 1.140337 & 0.076581 \\
\hline 11 & 6 & 0 & -1.687584 & -0.875902 & 0.033992 \\
\hline 12 & 1 & 0 & -1.731433 & -1.114296 & 1.113808 \\
\hline 13 & 1 & 0 & -2.677214 & -1.050808 & -0.400877 \\
\hline 14 & 1 & 0 & -0.987792 & -1.577402 & -0.432263 \\
\hline
\end{tabular}

\begin{tabular}{|c|c|c|c|c|c|c|c|c|c|c|}
\hline & & & 1 & & & 2 & & & 3 & \\
\hline & & & A & & & A & & & A & \\
\hline Frequ & dencies & $;--$ & 96.79 & & & 134.91 & & & 217.423 & \\
\hline Red. & masses & -- & 2.54 & & & 1.73 & & & 1.177 & \\
\hline $\operatorname{Frc}$ & onsts & -- & 0.01 & & & 0.01 & & & 0.032 & \\
\hline IR Ir & ten & -- & 0.11 & & & 2.49 & & & 0.438 & \\
\hline Atom & AN & X & $\mathrm{Y}$ & Z & $\mathrm{X}$ & $\mathrm{Y}$ & Z & $\mathrm{x}$ & Y & Z \\
\hline 1 & 6 & 0.01 & -0.11 & 0.02 & 0.01 & 0.07 & -0.04 & 0.00 & -0.04 & -0.03 \\
\hline 2 & 1 & 0.00 & -0.15 & 0.02 & 0.07 & 0.20 & -0.01 & -0.01 & -0.08 & -0.04 \\
\hline 3 & 1 & 0.10 & -0.12 & 0.06 & 0.06 & 0.06 & -0.18 & -0.03 & -0.03 & 0.03 \\
\hline 4 & 6 & -0.03 & -0.16 & 0.00 & -0.07 & -0.10 & -0.01 & 0.06 & 0.05 & -0.01 \\
\hline 5 & 1 & -0.22 & -0.40 & -0.03 & -0.16 & -0.32 & -0.04 & 0.18 & 0.17 & 0.01 \\
\hline 6 & 6 & 0.20 & 0.17 & 0.01 & -0.04 & 0.00 & 0.05 & 0.01 & 0.01 & 0.03 \\
\hline 7 & 1 & 0.41 & 0.43 & 0.05 & 0.04 & 0.22 & 0.09 & -0.11 & -0.10 & 0.01 \\
\hline 8 & 1 & 0.19 & 0.18 & -0.02 & -0.10 & -0.14 & 0.08 & 0.09 & 0.08 & 0.08 \\
\hline 9 & 7 & -0.02 & 0.03 & 0.02 & -0.04 & 0.06 & 0.08 & 0.00 & -0.03 & -0.03 \\
\hline
\end{tabular}




\begin{tabular}{|c|c|c|c|c|c|c|c|c|c|c|}
\hline 10 & 1 & 0.04 & 0.08 & 0.06 & -0.05 & -0.05 & 0.30 & 0.03 & 0.00 & -0.02 \\
\hline 11 & 6 & -0.16 & 0.05 & -0.05 & 0.13 & -0.03 & -0.09 & -0.06 & 0.00 & 0.03 \\
\hline 12 & 1 & -0.19 & 0.00 & -0.07 & 0.46 & -0.27 & -0.13 & 0.42 & -0.09 & 0.03 \\
\hline 13 & 1 & -0.17 & 0.17 & -0.07 & 0.01 & 0.01 & 0.16 & -0.30 & 0.20 & 0.49 \\
\hline 4 & 1 & -0.22 & 0.01 & -0.08 & 0.03 & 0.11 & -0.45 & -0.40 & -0.07 & -0.38 \\
\hline
\end{tabular}

thermodynamics:

Sum of electronic and zero-point Energies=

$-212.357569$

Sum of electronic and thermal Enthalpies=

$-212.350307$

Sum of electronic and thermal Free Energies=

$-212.387090$

Total free energy in solution:

with all non electrostatic terms

$(\mathrm{a} \cdot \mathrm{u})=$.

Optimized geometry, three lower frequencies, thermochemistry and PCM energy for allylmethylammonium.

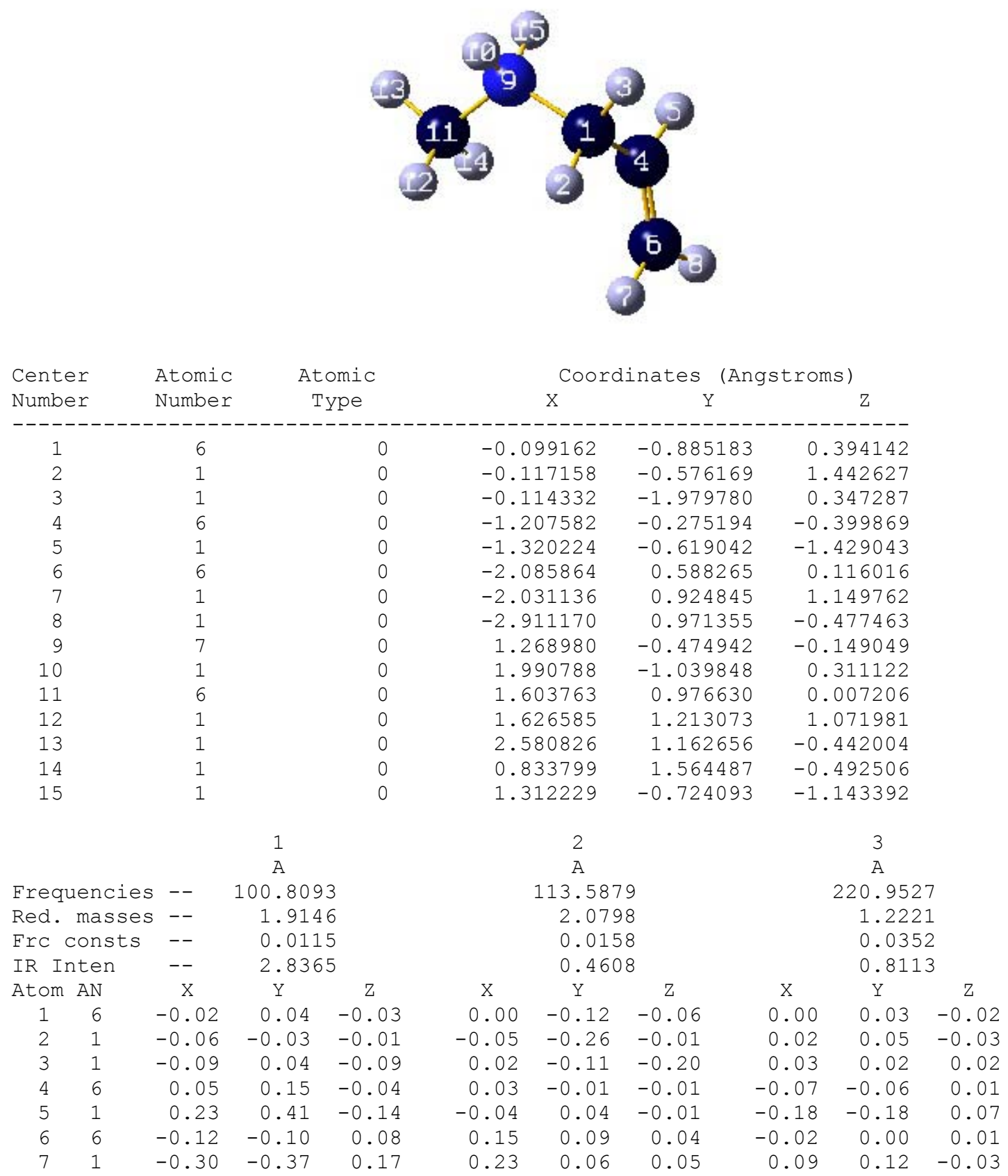




$\begin{array}{rrrrrrrrrrr}8 & 1 & -0.09 & -0.05 & 0.07 & 0.18 & 0.22 & 0.08 & -0.09 & -0.08 & 0.06 \\ 9 & 7 & 0.04 & -0.04 & 0.08 & 0.02 & -0.02 & 0.07 & 0.00 & 0.03 & -0.04 \\ 10 & 1 & -0.02 & 0.01 & 0.24 & 0.02 & 0.12 & 0.23 & -0.03 & -0.02 & -0.06 \\ 11 & 6 & 0.04 & -0.02 & -0.10 & -0.18 & 0.04 & -0.06 & 0.08 & 0.00 & 0.03 \\ 12 & 1 & -0.17 & 0.16 & -0.13 & -0.50 & 0.21 & -0.09 & -0.41 & 0.07 & 0.02 \\ 13 & 1 & 0.14 & -0.12 & 0.07 & -0.08 & 0.08 & 0.18 & 0.32 & -0.14 & 0.49 \\ 14 & 1 & 0.16 & -0.08 & -0.34 & -0.11 & -0.11 & -0.35 & 0.39 & 0.07 & -0.38 \\ 15 & 1 & 0.16 & -0.18 & 0.12 & 0.18 & -0.13 & 0.10 & -0.01 & 0.07 & -0.05\end{array}$

thermodynamics:

Sum of electronic and zero-point Energies=

$-212.713095$

Sum of electronic and thermal Enthalpies=

$-212.705745$

Sum of electronic and thermal Free Energies=

$-212.742811$

Total free energy in solution:

with all non electrostatic terms

$(\mathrm{a} \cdot \mathrm{u})=$.

Optimized geometry, three lower frequencies, thermochemistry and PCM energy for aniline.

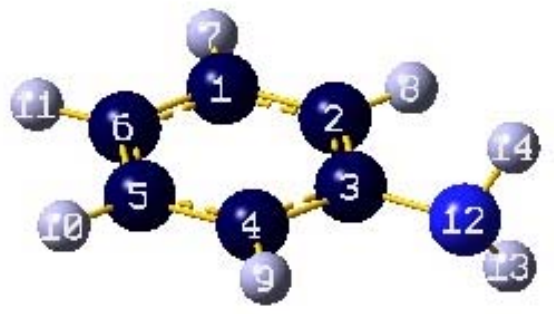

\begin{tabular}{|c|c|c|c|c|c|}
\hline Center & Atomic & Atomic & $\mathrm{COO}$ & inates (An & Eroms) \\
\hline Number & Number & Type & $\mathrm{X}$ & $Y$ & Z \\
\hline 1 & 6 & 0 & -1.154629 & 1.221147 & 0.000004 \\
\hline 2 & 6 & 0 & 0.240153 & 1.193270 & -0.000003 \\
\hline 3 & 6 & 0 & 0.928496 & -0.027067 & -0.000003 \\
\hline 4 & 6 & 0 & 0.195808 & -1.216697 & -0.000012 \\
\hline 5 & 6 & 0 & -1.199919 & -1.190818 & -0.000008 \\
\hline 6 & 6 & 0 & -1.878939 & 0.027698 & 0.000012 \\
\hline 7 & 1 & 0 & -1.675932 & 2.175807 & 0.000010 \\
\hline 8 & 1 & 0 & 0.804060 & 2.124795 & 0.000004 \\
\hline 9 & 1 & 0 & 0.740343 & -2.157077 & -0.000026 \\
\hline 10 & 1 & 0 & -1.756793 & -2.125023 & -0.000011 \\
\hline 11 & 1 & 0 & -2.966092 & 0.048787 & 0.000020 \\
\hline 12 & 7 & 0 & 2.364947 & -0.115231 & -0.000020 \\
\hline 13 & 1 & 0 & 2.756984 & 0.347275 & -0.817264 \\
\hline 14 & 1 & 0 & 2.756979 & 0.346854 & 0.817465 \\
\hline
\end{tabular}

\begin{tabular}{|c|c|c|c|c|c|c|c|c|c|}
\hline & \multicolumn{3}{|c|}{1} & \multicolumn{3}{|c|}{2} & \multicolumn{3}{|c|}{3} \\
\hline & \multicolumn{3}{|c|}{ A } & \multicolumn{3}{|c|}{ A } & \multicolumn{3}{|c|}{ A } \\
\hline Frequencies & -- & \multirow{2}{*}{\multicolumn{2}{|c|}{-267.8896}} & \multicolumn{3}{|c|}{217.9957} & \multicolumn{3}{|c|}{390.5811} \\
\hline Red. masses & -- & & & \multicolumn{3}{|c|}{3.5344} & \multicolumn{3}{|c|}{3.2446} \\
\hline Frc consts & -- & \multicolumn{2}{|c|}{0.0447} & \multicolumn{3}{|c|}{0.0990} & \multicolumn{3}{|c|}{0.2916} \\
\hline IR Inten & -- & \multicolumn{2}{|c|}{52.9417} & \multicolumn{3}{|c|}{0.8771} & \multicolumn{3}{|c|}{10.0969} \\
\hline Atom AN & X & Y & Z & X & $\mathrm{Y}$ & Z & X & Y & Z \\
\hline 16 & 0.00 & 0.00 & -0.02 & 0.00 & 0.00 & -0.01 & -0.09 & -0.02 & 0.00 \\
\hline 6 & 0.00 & 0.00 & -0.02 & 0.00 & 0.00 & 0.22 & -0.06 & 0.17 & 0.00 \\
\hline 6 & 0.00 & 0.00 & 0.00 & 0.00 & 0.00 & 0.16 & 0.01 & 0.18 & 0.00 \\
\hline 6 & 0.00 & 0.00 & 0.02 & 0.00 & 0.00 & 0.21 & 0.06 & 0.16 & 0.00 \\
\hline 6 & 0.00 & 0.00 & 0.03 & 0.00 & 0.00 & 0.00 & 0.08 & -0.02 & 0.00 \\
\hline 6 & 0.00 & 0.00 & 0.00 & 0.00 & 0.00 & -0.22 & -0.01 & -0.08 & 0.00 \\
\hline 1 & 0.00 & 0.00 & -0.03 & 0.00 & 0.00 & -0.09 & -0.20 & -0.08 & 0.00 \\
\hline 1 & 0.00 & 0.00 & -0.02 & 0.00 & 0.00 & 0.28 & -0.18 & 0.24 & 0.00 \\
\hline 9 & 0.00 & 0.00 & -0.02 & 0.00 & 0.00 & 0.25 & 0.19 & 0.24 & 0.00 \\
\hline 10 & 0.00 & 0.00 & 0.03 & 0.00 & 0.00 & -0.07 & 0.19 & -0.08 & 0.00 \\
\hline
\end{tabular}




\begin{tabular}{|c|c|c|c|c|c|c|c|c|c|c|}
\hline 11 & 1 & 0.00 & 0.00 & 0.00 & 0.00 & 0.00 & -0.50 & -0.01 & -0.15 & 0.00 \\
\hline 12 & 7 & 0.00 & 0.00 & -0.05 & 0.00 & 0.00 & -0.24 & -0.02 & -0.28 & 0.00 \\
\hline 13 & 1 & 0.00 & 0.63 & 0.31 & -0.27 & 0.06 & -0.33 & 0.20 & -0.47 & -0.01 \\
\hline 14 & 1 & 0.00 & -0.63 & 0.31 & 0.27 & -0.06 & -0.33 & 0.20 & -0.47 & 0.01 \\
\hline
\end{tabular}

thermodynamics:

Sum of electronic and zero-point Energies=

$-287.378111$

Sum of electronic and thermal Enthalpies=

$-287.371889$

Sum of electronic and thermal Free Energies=

$-287.406984$

Total free energy in solution:

with all non electrostatic terms (a.u.) $=\quad-287.502050$

Optimized geometry, three lower frequencies, thermochemistry and PCM energy for phenylammonium.

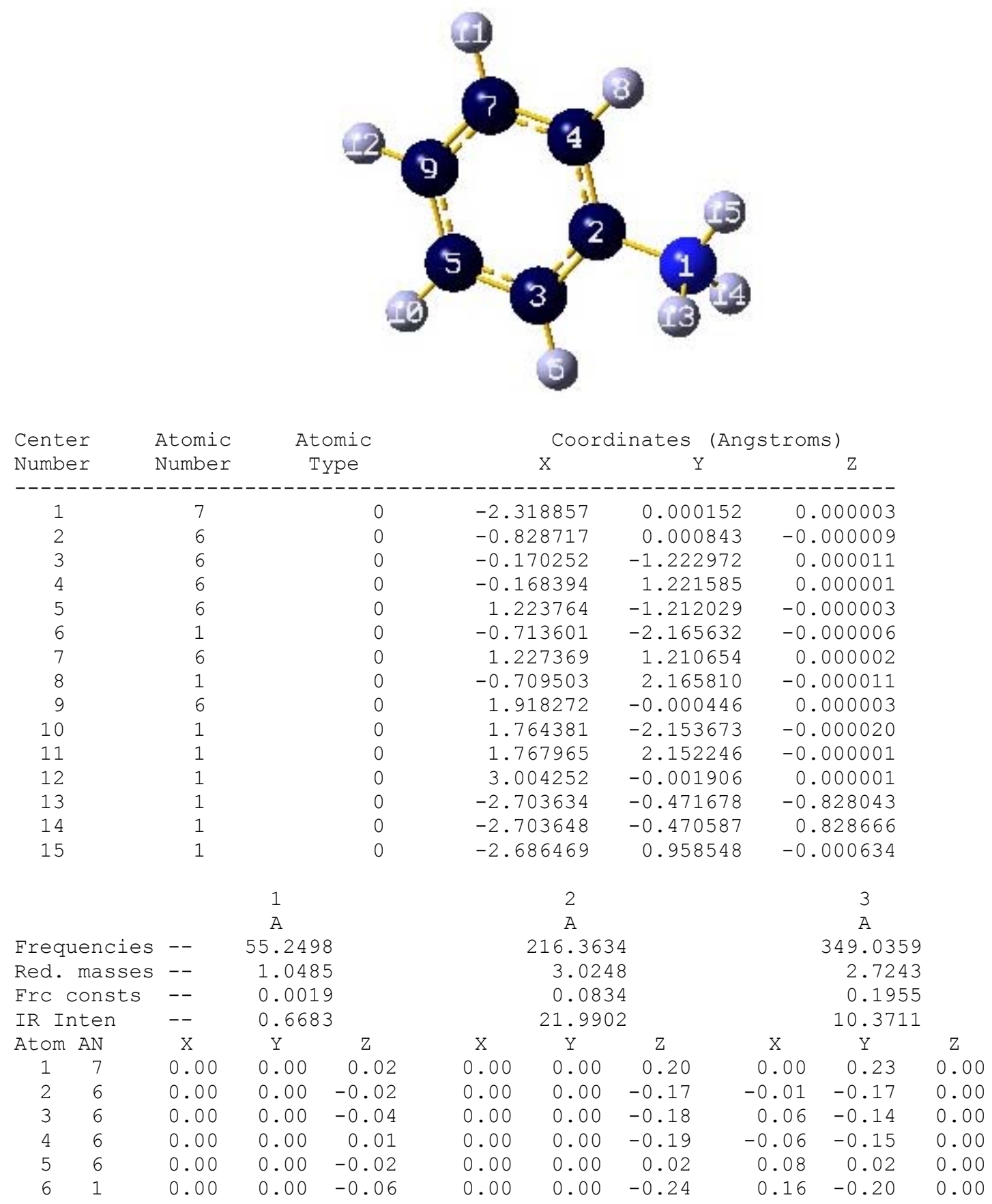




$\begin{array}{rrrrrrrrrrr}7 & 6 & 0.00 & 0.00 & 0.03 & 0.00 & 0.00 & 0.00 & -0.08 & 0.02 & 0.00 \\ 8 & 1 & 0.00 & 0.00 & 0.02 & 0.00 & 0.00 & -0.25 & -0.15 & -0.20 & 0.00 \\ 9 & 6 & 0.00 & 0.00 & 0.01 & 0.00 & 0.00 & 0.20 & 0.00 & 0.07 & 0.00 \\ 10 & 1 & 0.00 & 0.00 & -0.04 & 0.00 & 0.00 & 0.08 & 0.17 & 0.07 & 0.00 \\ 11 & 1 & 0.00 & 0.00 & 0.04 & 0.00 & 0.00 & 0.05 & -0.17 & 0.07 & 0.00 \\ 12 & 1 & 0.00 & 0.00 & 0.02 & 0.00 & 0.00 & 0.46 & 0.00 & 0.14 & 0.00 \\ 13 & 1 & -0.02 & -0.50 & 0.31 & -0.25 & 0.12 & 0.24 & -0.19 & 0.38 & 0.00 \\ 14 & 1 & 0.02 & 0.50 & 0.31 & 0.25 & -0.12 & 0.24 & -0.19 & 0.38 & 0.00 \\ 15 & 1 & 0.00 & 0.00 & -0.55 & 0.00 & 0.00 & 0.46 & 0.37 & 0.37 & 0.00\end{array}$

thermodynamics:

Sum of electronic and zero-point Energies=

Sum of electronic and thermal Enthalpies=

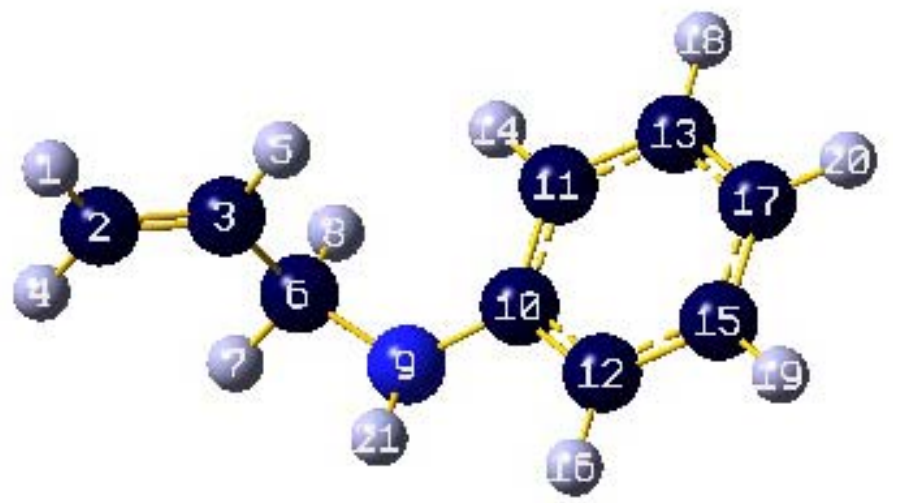

\begin{tabular}{|c|c|c|c|c|c|}
\hline Center & Atomic & Atomic & $\mathrm{COO}$ & nates & (roms) \\
\hline Number & Number & Type & $\mathrm{x}$ & Y & Z \\
\hline 1 & 1 & 0 & -4.237885 & 0.577439 & 1.803272 \\
\hline 2 & 6 & 0 & -3.859856 & 0.226944 & 0.845929 \\
\hline 3 & 6 & 0 & -2.590366 & 0.417443 & 0.478258 \\
\hline 4 & 1 & 0 & -4.572068 & -0.283251 & 0.197955 \\
\hline 5 & 1 & 0 & -1.900805 & 0.926981 & 1.152545 \\
\hline 6 & 6 & 0 & -2.007328 & -0.052456 & -0.829214 \\
\hline 7 & 1 & 0 & -2.757123 & -0.625966 & -1.386992 \\
\hline 8 & 1 & 0 & -1.745520 & 0.801083 & -1.466349 \\
\hline 9 & 7 & 0 & -0.828123 & -0.891475 & -0.683841 \\
\hline 10 & 6 & 0 & 0.416701 & -0.392817 & -0.310221 \\
\hline 11 & 6 & 0 & 0.770645 & 0.961135 & -0.44702 \\
\hline 12 & 6 & 0 & 1.390840 & -1.285560 & 0.178972 \\
\hline 13 & 6 & 0 & 2.055454 & 1.392449 & -0.116021 \\
\hline 14 & 1 & 0 & 0.050130 & 1.686791 & -0.81240 \\
\hline 15 & 6 & 0 & 2.666407 & -0.843009 & 0.507318 \\
\hline 16 & 1 & 0 & 1.137991 & -2.339042 & 0.290592 \\
\hline 17 & 6 & 0 & 3.015203 & 0.502493 & 0.36242 \\
\hline 18 & 1 & 0 & 2.301263 & 2.445795 & -0.23280 \\
\hline 19 & 1 & 0 & 3.395464 & -1.558362 & 0.88172 \\
\hline 20 & 1 & 0 & 4.012369 & 0.847886 & 0.62113 \\
\hline 21 & 1 & 0 & -1.033157 & -1.798764 & -0.28434 \\
\hline & & & 2 & & 3 \\
\hline & & & A & & A \\
\hline
\end{tabular}




\begin{tabular}{|c|c|c|c|c|c|c|c|c|c|c|}
\hline \multicolumn{2}{|c|}{ Frequencies } & -- & \multicolumn{2}{|c|}{43.5075} & \multicolumn{3}{|c|}{54.2294} & \multicolumn{3}{|c|}{104.1748} \\
\hline Red. & masses & -- & $3.73^{7}$ & & & 3.88 & & & 2.17 & \\
\hline Frc c & ronsts & -- & 0.00 & & & 0.00 & & & 0.01. & \\
\hline IR In & ten & -- & 0.36 & & & 0.19 & & & 0.16 & \\
\hline Atom & AN & $\mathrm{X}$ & $\mathrm{Y}$ & Z & $\mathrm{X}$ & $\mathrm{Y}$ & Z & $\mathrm{X}$ & $\mathrm{Y}$ & Z \\
\hline 1 & 1 & -0.12 & 0.32 & -0.19 & 0.36 & 0.26 & 0.16 & -0.09 & 0.06 & -0.02 \\
\hline 2 & 6 & -0.08 & 0.19 & -0.13 & 0.21 & 0.16 & 0.14 & -0.09 & 0.15 & -0.06 \\
\hline 3 & 6 & -0.04 & 0.04 & -0.05 & 0.18 & 0.03 & -0.03 & 0.01 & -0.18 & 0.13 \\
\hline 4 & 1 & -0.08 & 0.19 & -0.13 & 0.09 & 0.17 & 0.26 & -0.19 & 0.53 & -0.25 \\
\hline 5 & 1 & -0.04 & 0.05 & -0.05 & 0.30 & 0.03 & -0.14 & 0.10 & -0.55 & 0.32 \\
\hline 6 & 6 & 0.01 & -0.11 & 0.02 & 0.00 & -0.10 & -0.06 & 0.01 & -0.02 & 0.07 \\
\hline 7 & 1 & 0.03 & -0.17 & 0.07 & -0.08 & -0.14 & 0.08 & 0.00 & 0.05 & 0.00 \\
\hline 8 & 1 & 0.03 & -0.18 & -0.07 & -0.08 & -0.15 & -0.18 & -0.02 & 0.07 & 0.18 \\
\hline 9 & 7 & 0.01 & -0.08 & 0.14 & 0.01 & -0.10 & -0.15 & 0.03 & -0.01 & -0.06 \\
\hline 10 & 6 & 0.01 & -0.05 & 0.07 & -0.02 & -0.06 & -0.08 & 0.02 & -0.01 & -0.04 \\
\hline 11 & 6 & -0.08 & -0.01 & 0.18 & -0.09 & -0.04 & -0.02 & 0.02 & -0.01 & -0.06 \\
\hline 12 & 6 & 0.11 & -0.04 & -0.10 & 0.00 & -0.03 & -0.06 & 0.01 & 0.01 & 0.00 \\
\hline 13 & 6 & -0.07 & 0.03 & 0.11 & -0.13 & 0.03 & 0.09 & 0.01 & 0.01 & -0.03 \\
\hline 14 & 1 & -0.16 & -0.02 & 0.34 & -0.10 & -0.07 & -0.04 & 0.03 & -0.02 & -0.10 \\
\hline 15 & 6 & 0.11 & 0.00 & -0.17 & -0.05 & 0.04 & 0.04 & 0.00 & 0.02 & 0.03 \\
\hline 16 & 1 & 0.18 & -0.07 & -0.20 & 0.05 & -0.04 & -0.11 & 0.01 & 0.01 & 0.02 \\
\hline 17 & 6 & 0.02 & 0.04 & -0.07 & -0.12 & 0.06 & 0.13 & 0.00 & 0.02 & 0.02 \\
\hline 18 & 1 & -0.15 & 0.05 & 0.20 & -0.18 & 0.04 & 0.14 & 0.00 & 0.01 & -0.04 \\
\hline 19 & 1 & 0.19 & 0.00 & -0.31 & -0.03 & 0.06 & 0.07 & -0.01 & 0.03 & 0.07 \\
\hline 20 & 1 & 0.02 & 0.07 & -0.12 & -0.16 & 0.11 & 0.21 & -0.01 & 0.03 & 0.05 \\
\hline 21 & 1 & 0.03 & -0.08 & 0.15 & 0.03 & -0.10 & -0.15 & 0.06 & -0.05 & -0.13 \\
\hline
\end{tabular}

thermodynamics:

Sum of electronic and zero-point Energies=

$-403.981217$

Sum of electronic and thermal Enthalpies=

$-403.970954$

Sum of electronic and thermal Free Energies=

$-404.016621$

Total free energy in solution:

with all non electrostatic terms

$($ a.u. $)=-404.163294$

Optimized geometry, three lower frequencies, thermochemistry and PCM energy for allylphenylammonium.

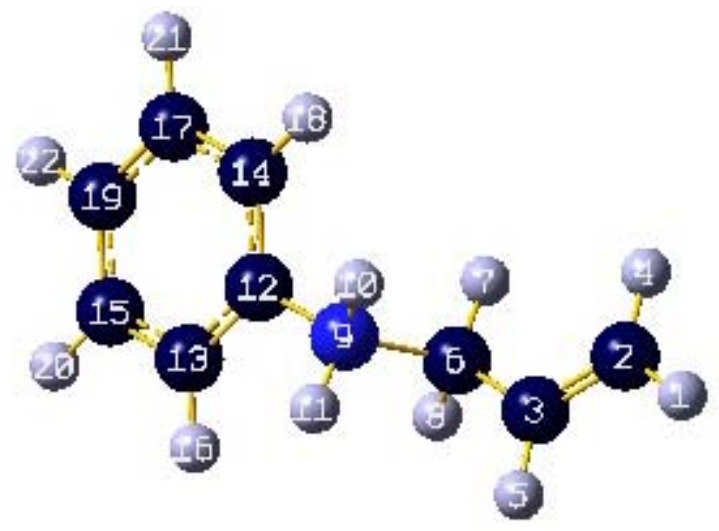

\begin{tabular}{|c|c|c|c|c|c|}
\hline \multirow{2}{*}{$\begin{array}{l}\text { Center } \\
\text { Number }\end{array}$} & \multirow{2}{*}{$\begin{array}{l}\text { Atomic } \\
\text { Number }\end{array}$} & \multirow{2}{*}{$\begin{array}{c}\text { Atomic } \\
\text { Type }\end{array}$} & \multicolumn{3}{|c|}{ Coordinates (Angstroms) } \\
\hline & & & $\mathrm{X}$ & $Y$ & Z \\
\hline 1 & 1 & 0 & 5.176716 & 0.410750 & 0.107047 \\
\hline 2 & 6 & 0 & 4.146989 & 0.567221 & -0.201652 \\
\hline 3 & 6 & 0 & 3.238340 & -0.406542 & -0.099554 \\
\hline 4 & 1 & 0 & 3.905942 & 1.544210 & -0.617277 \\
\hline 5 & 1 & 0 & 3.526877 & -1.384302 & 0.287833 \\
\hline 6 & 6 & 0 & 1.820879 & -0.245007 & -0.543441 \\
\hline 7 & 1 & 0 & 1.646152 & 0.696462 & -1.069230 \\
\hline 8 & 1 & 0 & 1.467995 & -1.069217 & -1.170297 \\
\hline 9 & 7 & 0 & 0.881401 & -0.226752 & 0.667669 \\
\hline
\end{tabular}




\begin{tabular}{|c|c|c|c|c|c|c|c|c|c|c|}
\hline \multicolumn{2}{|l|}{10} & \multicolumn{2}{|l|}{1} & 0 & \multicolumn{2}{|c|}{1.183326} & 0.533791 & \multicolumn{3}{|c|}{1.287375} \\
\hline 11 & & \multicolumn{2}{|l|}{1} & 0 & \multicolumn{2}{|c|}{1.029786} & \multicolumn{2}{|c|}{-1.087049} & \multicolumn{2}{|c|}{1.207075} \\
\hline 12 & & \multicolumn{2}{|l|}{6} & 0 & \multicolumn{2}{|c|}{-0.554524} & -0.082069 & \multicolumn{3}{|c|}{0.350184} \\
\hline 13 & & 6 & & 0 & \multicolumn{2}{|c|}{-1.306144} & -1.228651 & \multicolumn{3}{|c|}{0.117024} \\
\hline 14 & & 6 & & 0 & \multicolumn{2}{|c|}{-1.093072} & 1.197151 & \multicolumn{3}{|c|}{0.262730} \\
\hline 15 & & \multicolumn{2}{|l|}{6} & 0 & \multicolumn{2}{|c|}{-2.652582} & -1.081560 & -0 & 215008 & \\
\hline 16 & & 1 & & 0 & -0.8 & 65587 & -2.220 & & 194657 & \\
\hline 17 & & 6 & & 0 & -2.4 & 41004 & 1.326 & -0 & 070409 & \\
\hline 18 & & 1 & & 0 & -0.4 & 87628 & 2.081 & & 453542 & \\
\hline 19 & & 6 & & 0 & -3.2 & 16470 & 0.191 & -0 & 309369 & \\
\hline 20 & & 1 & & 0 & -3.2 & 58766 & -1.964 & -0 & 394383 & \\
\hline 21 & & 1 & & 0 & -2.8 & 82715 & 2.316 & -0 & 137491 & \\
\hline 22 & & 1 & & 0 & -4.2 & 66371 & 0.299 & -0 & 565564 & \\
\hline & & & 1 & & & 2 & & & 3 & \\
\hline & & & $A$ & & & A & & & A & \\
\hline Erequ & lencies & $5--$ & 45.18 & & & 56.16 & & & 93.45 & \\
\hline Red. & masses & $5--$ & 2.62 & & & 3.21 & & & 3.09 & \\
\hline $\operatorname{Erc}$ & onsts & -- & 0.003 & & & 0.00 & & & 0.01 & \\
\hline IR $\mathrm{Ir}$ & ten & -- & 0.65 & & & 1.96 & & & 0.36 & \\
\hline Atom & AN & $\mathrm{X}$ & $\mathrm{Y}$ & Z & $\mathrm{X}$ & $\mathrm{Y}$ & Z & $\mathrm{X}$ & $\mathrm{Y}$ & $\mathrm{Z}$ \\
\hline 1 & 1 & 0.00 & -0.04 & 0.17 & -0.11 & 0.42 & -0.07 & -0.08 & 0.18 & 0.33 \\
\hline 2 & 6 & 0.01 & 0.04 & 0.18 & -0.13 & 0.22 & -0.07 & -0.05 & 0.06 & 0.17 \\
\hline 3 & 6 & 0.00 & 0.01 & -0.09 & 0.02 & 0.09 & 0.04 & 0.04 & -0.04 & 0.05 \\
\hline 4 & 1 & 0.02 & 0.14 & 0.42 & -0.29 & 0.14 & -0.17 & -0.10 & 0.02 & 0.11 \\
\hline 5 & 1 & -0.01 & -0.08 & -0.32 & 0.18 & 0.17 & 0.14 & 0.09 & 0.00 & 0.13 \\
\hline 6 & 6 & 0.01 & 0.13 & -0.07 & -0.01 & -0.16 & 0.03 & 0.07 & -0.19 & -0.10 \\
\hline 7 & 1 & 0.03 & 0.23 & 0.11 & -0.13 & -0.24 & -0.06 & 0.08 & -0.30 & -0.31 \\
\hline 8 & 1 & 0.00 & 0.25 & -0.23 & 0.10 & -0.27 & 0.11 & 0.14 & -0.33 & 0.06 \\
\hline 9 & 7 & 0.01 & -0.09 & -0.07 & 0.00 & -0.16 & 0.03 & 0.02 & 0.06 & -0.14 \\
\hline 10 & 1 & 0.01 & -0.20 & 0.07 & 0.02 & -0.18 & 0.05 & 0.04 & 0.17 & -0.28 \\
\hline 11 & 1 & 0.01 & -0.19 & -0.22 & -0.04 & -0.18 & 0.02 & 0.00 & 0.16 & 0.03 \\
\hline 12 & 6 & 0.00 & -0.05 & -0.03 & 0.01 & -0.08 & 0.02 & 0.02 & 0.04 & -0.13 \\
\hline 13 & 6 & -0.06 & -0.03 & 0.09 & -0.07 & -0.03 & 0.02 & 0.02 & 0.03 & -0.09 \\
\hline 14 & 6 & 0.06 & -0.03 & -0.12 & 0.10 & -0.05 & 0.00 & -0.02 & 0.03 & -0.06 \\
\hline 15 & 6 & -0.07 & 0.00 & 0.13 & -0.05 & 0.06 & -0.01 & -0.01 & 0.01 & 0.05 \\
\hline 16 & 1 & -0.10 & -0.05 & 0.15 & -0.14 & -0.06 & 0.03 & 0.05 & 0.04 & -0.15 \\
\hline 17 & 6 & 0.05 & 0.01 & -0.08 & 0.11 & 0.05 & -0.03 & -0.06 & 0.01 & 0.08 \\
\hline 18 & 1 & 0.11 & -0.05 & -0.21 & 0.16 & -0.09 & 0.01 & -0.02 & 0.04 & -0.10 \\
\hline 19 & 6 & -0.01 & 0.02 & 0.05 & 0.04 & 0.10 & -0.03 & -0.06 & -0.01 & 0.15 \\
\hline 20 & 1 & -0.12 & 0.02 & 0.23 & -0.11 & 0.10 & -0.01 & -0.01 & 0.00 & 0.09 \\
\hline 21 & 1 & 0.10 & 0.02 & -0.14 & 0.19 & 0.08 & -0.04 & -0.09 & 0.00 & 0.15 \\
\hline 22 & 1 & -0.02 & 0.05 & 0.08 & 0.05 & 0.17 & -0.05 & -0.09 & -0.03 & 0.28 \\
\hline
\end{tabular}

thermodynamics:

Sum of electronic and zero-point Energie

Sum of electronic and thermal Enthalpies=

$-404.331811$

Sum of electronic and thermal Enthalpies=

Sum of electronic and thermal Free Energies=

$-404.321417$

Total free energy in solution:

with all non electrostatic terms

$-404.367624$

$(\mathrm{a} \cdot \mathrm{u} \cdot)=$

$-404.602134$

Optimized geometry, three lower frequencies, thermochemistry and PCM energy for triflate anion.

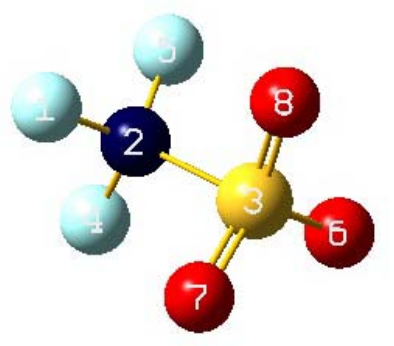




\begin{tabular}{|c|c|c|c|c|c|c|c|c|c|c|}
\hline \multirow{2}{*}{\multicolumn{2}{|c|}{$\begin{array}{l}\text { Center } \\
\text { Number }\end{array}$}} & \multirow{2}{*}{$\begin{array}{l}\text { Atomic } \\
\text { Number }\end{array}$} & \multirow{2}{*}{\multicolumn{2}{|c|}{$\begin{array}{l}\text { Atomic } \\
\text { Type }\end{array}$}} & \multicolumn{6}{|c|}{ Coordinates (Angstroms) } \\
\hline & & & & & \multicolumn{2}{|r|}{$\mathrm{X}$} & $\mathrm{Y}$ & \multicolumn{2}{|c|}{ Z } & \\
\hline 1 & & 9 & & 0 & -1. & 51836 & 0.672 & & 057316 & \\
\hline 2 & & 6 & & 0 & -0 & 46975 & -0.000 & & 000004 & \\
\hline 3 & & 16 & & 0 & 0. & 32879 & 0.000 & -0 & 000010 & \\
\hline 4 & & 9 & & 0 & -1.2 & 51849 & -1.251 & & 053777 & \\
\hline 5 & & 9 & & 0 & -1. & 51975 & 0.579 & -1 & 111054 & \\
\hline 6 & & 8 & & 0 & 1.2 & 48301 & -0.788 & -1 & 208419 & \\
\hline 7 & & 8 & & 0 & 1.2 & 48245 & -0.652 & & 286704 & \\
\hline \multirow{3}{*}{\multicolumn{2}{|c|}{8}} & 8 & & 0 & 1.2 & 48046 & 1.440 & -0 & 078312 & \\
\hline & & \multicolumn{3}{|c|}{1} & \multicolumn{3}{|c|}{2} & \multicolumn{3}{|c|}{3} \\
\hline & & \multicolumn{3}{|c|}{ A } & \multicolumn{3}{|c|}{ A } & \multicolumn{3}{|c|}{ A } \\
\hline \multicolumn{2}{|c|}{ Frequencies } & -- & \multirow{2}{*}{\multicolumn{2}{|c|}{$\begin{array}{l}60.3130 \\
17.4499\end{array}$}} & \multicolumn{3}{|c|}{188.3279} & \multicolumn{3}{|c|}{189.2404} \\
\hline \multicolumn{2}{|c|}{ Red. masses } & -- & & & \multicolumn{3}{|c|}{17.2347} & \multicolumn{3}{|c|}{17.2350} \\
\hline \multicolumn{2}{|c|}{ Frc consts } & -- & \multicolumn{2}{|c|}{0.0374} & \multicolumn{3}{|c|}{0.3601} & \multicolumn{3}{|c|}{0.3637} \\
\hline \multicolumn{2}{|c|}{ IR Inten } & -- & \multicolumn{2}{|c|}{0.0000} & \multicolumn{3}{|c|}{1.1748} & \multicolumn{3}{|c|}{1.1845} \\
\hline Atom & AN & X & Y & Z & $\mathrm{x}$ & $\mathrm{Y}$ & Z & $\mathrm{X}$ & $\mathrm{Y}$ & Z \\
\hline 1 & 9 & 0.00 & 0.34 & -0.22 & 0.46 & 0.02 & 0.06 & -0.21 & -0.04 & -0.01 \\
\hline 2 & 6 & 0.00 & 0.00 & 0.00 & 0.00 & -0.02 & -0.12 & 0.00 & 0.12 & -0.02 \\
\hline 3 & 16 & 0.00 & 0.00 & 0.00 & 0.00 & -0.01 & -0.08 & 0.00 & 0.08 & -0.01 \\
\hline 4 & 9 & 0.00 & 0.02 & 0.40 & -0.05 & 0.01 & 0.03 & 0.51 & -0.07 & 0.01 \\
\hline 5 & 9 & 0.00 & -0.36 & -0.19 & -0.41 & -0.01 & 0.06 & -0.30 & -0.04 & 0.02 \\
\hline 6 & 8 & 0.00 & 0.35 & -0.23 & 0.56 & 0.02 & 0.04 & -0.26 & -0.02 & -0.02 \\
\hline 7 & 8 & 0.00 & -0.37 & -0.19 & -0.51 & -0.02 & 0.04 & -0.36 & -0.02 & 0.02 \\
\hline 8 & 8 & 0.00 & 0.02 & 0.41 & -0.06 & 0.00 & 0.01 & 0.62 & -0.05 & 0.00 \\
\hline
\end{tabular}

thermodynamics:

Sum of electronic and zero-point Energies=

$-961.264666$

Sum of electronic and thermal Enthalpies=

$-961.256502$

Sum of electronic and thermal Free Energies=

$-961.297178$

Total free energy in solution:

with all non electrostatic terms

$(\mathrm{a} \cdot \mathrm{u})=$.

Total free energy in solution:

with all non electrostatic terms

$(\mathrm{a} \cdot \mathrm{u} \cdot)=-961.343649$

\section{DCPB-H}

Optimized geometry, three lower frequencies, thermochemistry and PCM energy for $\mathbf{1 a}$.

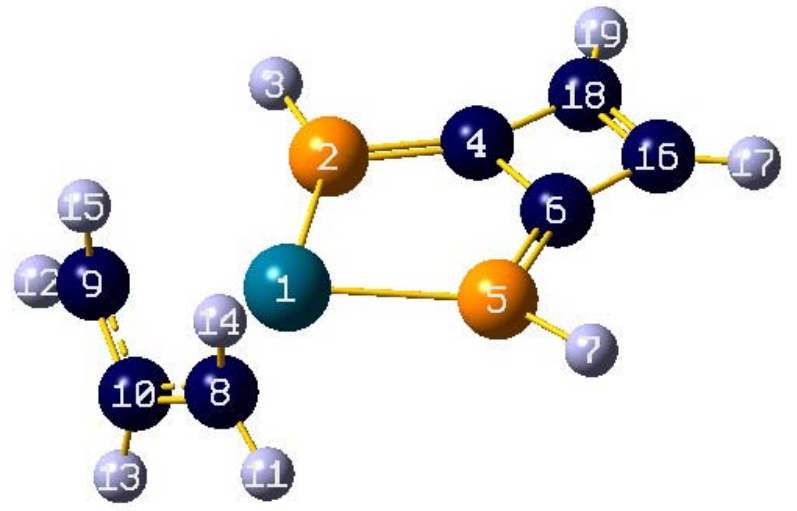

\begin{tabular}{|c|c|c|c|c|c|}
\hline Center & Atomic & Atomic & \multicolumn{3}{|c|}{ Coordinates (Angstroms) } \\
\hline Number & Number & Type & $\mathrm{X}$ & Y & Z \\
\hline 1 & 46 & 0 & -0.927359 & -0.000047 & 0.010293 \\
\hline
\end{tabular}




\begin{tabular}{|c|c|c|c|c|c|}
\hline 2 & 15 & 0 & 0.829285 & 1.593203 & -0.014881 \\
\hline 3 & 1 & 0 & 1.108741 & 2.976755 & 0.001279 \\
\hline 4 & 6 & 0 & 2.277390 & 0.753298 & -0.008060 \\
\hline 5 & 15 & 0 & 0.829360 & -1.593249 & -0.015127 \\
\hline 6 & 6 & 0 & 2.277419 & -0.753261 & -0.007952 \\
\hline 7 & 1 & 0 & 1.108931 & -2.976773 & 0.001567 \\
\hline 8 & 6 & 0 & -2.731655 & -1.208743 & 0.201704 \\
\hline 9 & 6 & 0 & -2.731391 & 1.208952 & 0.201304 \\
\hline 10 & 6 & 0 & -3.060377 & 0.000023 & -0.443926 \\
\hline 11 & 1 & 0 & -2.796358 & -2.146678 & -0.343062 \\
\hline 12 & 1 & 0 & -2.795874 & 2.146702 & -0.343809 \\
\hline 13 & 1 & 0 & -3.297838 & -0.000135 & -1.506477 \\
\hline 14 & 1 & 0 & -2.773564 & -1.283022 & 1.288796 \\
\hline 15 & 1 & 0 & -2.773433 & 1.283644 & 1.288362 \\
\hline 16 & 6 & 0 & 3.732720 & -0.690579 & -0.005224 \\
\hline 17 & 1 & 0 & 4.508762 & -1.447944 & -0.002503 \\
\hline 18 & 6 & 0 & 3.732692 & 0.690680 & -0.005312 \\
\hline 19 & 1 & 0 & 4.508702 & 1.448078 & -0.002711 \\
\hline
\end{tabular}

\begin{tabular}{|c|c|c|c|c|c|c|c|c|c|c|}
\hline & & & 1 & & & 2 & & & 3 & \\
\hline & & & A & & & A & & & A & \\
\hline Freq & encies & -- & 38.580 & & & 92.59 & & & 100.146 & \\
\hline Red. & masses & -- & 6.023 & & & 2.93 & & & 4.926 & \\
\hline Frc & onsts & -- & 0.005 & & & 0.01 & & & 0.029 & \\
\hline IR I & ten & -- & 4.243 & & & 0.18 & & & 4.415 & \\
\hline Atom & AN & $\mathrm{X}$ & Y & Z & $\mathrm{x}$ & $\mathrm{Y}$ & Z & $\mathrm{x}$ & $\mathrm{Y}$ & Z \\
\hline 1 & 46 & 0.00 & 0.00 & 0.06 & 0.00 & -0.01 & 0.00 & 0.00 & 0.08 & 0.00 \\
\hline 2 & 15 & 0.00 & 0.00 & 0.16 & 0.00 & 0.00 & -0.08 & 0.08 & 0.05 & 0.00 \\
\hline 3 & 1 & 0.00 & 0.00 & 0.03 & 0.00 & 0.00 & -0.17 & 0.20 & 0.03 & -0.01 \\
\hline 4 & 6 & 0.00 & 0.00 & -0.04 & 0.00 & 0.00 & -0.05 & 0.04 & -0.04 & 0.00 \\
\hline 5 & 15 & 0.00 & 0.00 & 0.16 & 0.00 & 0.00 & 0.08 & -0.08 & 0.05 & 0.00 \\
\hline 6 & 6 & 0.00 & 0.00 & -0.04 & 0.00 & 0.00 & 0.05 & -0.04 & -0.04 & 0.00 \\
\hline 7 & 1 & 0.00 & 0.00 & 0.03 & 0.00 & 0.00 & 0.17 & -0.20 & 0.03 & 0.01 \\
\hline 8 & 6 & -0.03 & 0.00 & -0.20 & 0.00 & -0.03 & -0.24 & 0.22 & -0.17 & -0.02 \\
\hline 9 & 6 & -0.03 & 0.00 & -0.20 & 0.00 & -0.03 & 0.24 & -0.22 & -0.17 & 0.02 \\
\hline 10 & 6 & 0.06 & 0.00 & -0.24 & 0.00 & 0.10 & 0.00 & 0.00 & -0.21 & 0.00 \\
\hline 11 & 1 & 0.05 & 0.00 & -0.21 & 0.02 & 0.08 & -0.43 & 0.39 & -0.17 & -0.04 \\
\hline 12 & 1 & 0.05 & 0.00 & -0.21 & -0.02 & 0.08 & 0.43 & -0.39 & -0.17 & 0.04 \\
\hline 13 & 1 & 0.22 & 0.00 & -0.28 & 0.00 & 0.31 & 0.00 & 0.00 & -0.24 & 0.00 \\
\hline 14 & 1 & -0.18 & 0.00 & -0.20 & -0.04 & -0.26 & -0.26 & 0.21 & -0.21 & -0.02 \\
\hline 15 & 1 & -0.18 & 0.00 & -0.20 & 0.04 & -0.26 & 0.26 & -0.21 & -0.21 & 0.02 \\
\hline 16 & 6 & 0.00 & 0.00 & -0.26 & 0.00 & 0.00 & 0.06 & -0.04 & -0.12 & 0.00 \\
\hline 17 & 1 & 0.00 & 0.00 & -0.39 & 0.00 & 0.01 & 0.13 & -0.08 & -0.16 & 0.01 \\
\hline 18 & 6 & 0.00 & 0.00 & -0.26 & 0.00 & 0.00 & -0.06 & 0.04 & -0.12 & 0.00 \\
\hline 19 & 1 & 0.00 & 0.00 & -0.39 & 0.00 & 0.01 & -0.13 & 0.08 & -0.16 & -0.01 \\
\hline
\end{tabular}

thermodynamics:

Sum of electronic and zero-point Energies=

$-1081.011713$

Sum of electronic and thermal Enthalpies=

$-1080.998889$

Sum of electronic and thermal Free Energies=

$-1081.050585$

Total free energy in solution:

with all non electrostatic terms $\quad($ a.u. $)=-1081.209887$

Optimized geometry, three lower frequencies, thermochemistry and PCM energy for $\mathbf{T S}_{\mathbf{1 a - 2 a}}$. 


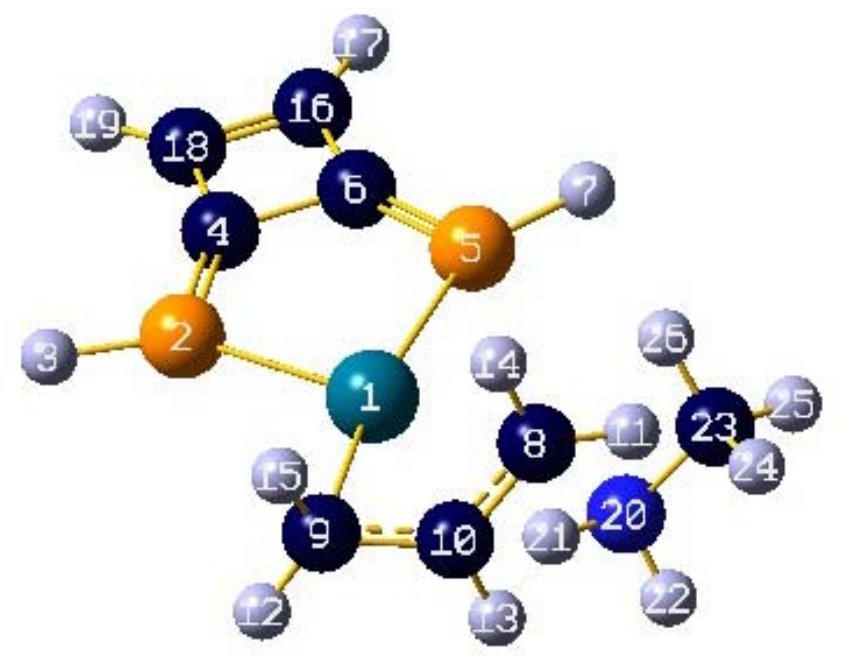

\begin{tabular}{|c|c|c|c|c|c|c|c|c|c|c|}
\hline \multirow{2}{*}{\multicolumn{2}{|c|}{$\begin{array}{l}\text { Center } \\
\text { Number }\end{array}$}} & \multirow{2}{*}{$\begin{array}{l}\text { Atomic } \\
\text { Number }\end{array}$} & \multirow{2}{*}{\multicolumn{2}{|c|}{$\begin{array}{l}\text { Atomic } \\
\text { Type }\end{array}$}} & \multicolumn{6}{|c|}{ Coordinates (Angstroms) } \\
\hline & & & & & & $\mathrm{x}$ & $\mathrm{Y}$ & & z & \\
\hline 1 & & 46 & & 0 & 0.1 & 37918 & -0.40399 & -0 & 63682 & \\
\hline 2 & & 15 & & 0 & -1.9 & 24336 & -1.41496 & & 392900 & \\
\hline 3 & & 1 & & 0 & -2.5 & 74594 & -2.62952 & 0 & 16894 & \\
\hline 4 & & 6 & & 0 & -3.1 & 12025 & -0.23577 & 0 & 313234 & \\
\hline 5 & & 15 & & 0 & -1.1 & 56198 & 1.60329 & -0 & 353068 & \\
\hline 6 & & 6 & & 0 & -2.7 & 47795 & 1.18607 & -0 & 30960 & \\
\hline 7 & & 1 & & 0 & -1.1 & 67352 & 2.99003 & -0 & 541135 & \\
\hline 8 & & 6 & & 0 & 2.5 & 75064 & 0.33959 & -0 & 90461 & \\
\hline 9 & & 6 & & 0 & 1.6 & 15466 & -1.93427 & -0 & 89033 & \\
\hline 10 & & 6 & & 0 & 2.1 & 85627 & -0.83331 & -0 & 773584 & \\
\hline 11 & & 1 & & 0 & 2.6 & 31215 & 1.27491 & -0 & 527496 & \\
\hline 12 & & 1 & & 0 & 1.3 & 77112 & -2.83917 & -0 & 542178 & \\
\hline 13 & & 1 & & 0 & 2.2 & 73095 & -0.85838 & -1 & 358438 & \\
\hline 14 & & 1 & & 0 & 2.4 & 96674 & 0.40489 & & 990240 & \\
\hline 15 & & 1 & & 0 & 1.8 & 01074 & -2.07518 & & 976308 & \\
\hline 16 & & 6 & & 0 & -4.1 & 62929 & 1.51169 & & 95355 & \\
\hline 17 & & 1 & & 0 & -4.7 & 27149 & 2.43051 & -0 & 20517 & \\
\hline 18 & & 6 & & 0 & -4.4 & 96542 & 0.20946 & & 10172 & \\
\hline 19 & & 1 & & 0 & -5.4 & 26113 & -0.29943 & & 540221 & \\
\hline 20 & & 7 & & 0 & 4.7 & 59498 & 0.30711 & & 22129 & \\
\hline 21 & & 1 & & 0 & 4.9 & 59930 & -0.56017 & & 616658 & \\
\hline 22 & & 1 & & 0 & 5.1 & 24772 & 0.20147 & -0 & 822671 & \\
\hline 23 & & 6 & & 0 & 5.3 & 51851 & 1.46475 & & 300540 & \\
\hline 24 & & 1 & & 0 & 6.4 & 43507 & 1.39666 & & 386219 & \\
\hline 25 & & 1 & & 0 & 5.1 & 03576 & 2.37395 & & 245576 & \\
\hline 26 & & 1 & & 0 & 4.9 & 29241 & 1.54906 & & 305754 & \\
\hline & & & 1 & & & 2 & & & 3 & \\
\hline & & & A & & & A & & & A & \\
\hline Freq & dencies & $s--$ & -208.178 & & & 21.95 & & & 28.046 & \\
\hline Red. & masses & $s--$ & 5.849 & & & 5.10 & & & 2.819 & \\
\hline Frc & consts & -- & 0.149 & & & 0.00 & & & 0.001 & \\
\hline IR I & nten & -- & 610.757 & & & 2.78 & & & 2.699 & \\
\hline Atom & AN & X & $\mathrm{Y}$ & Z & X & Y & Z & X & Y & Z \\
\hline 1 & 46 & -0.01 & -0.02 & -0.01 & 0.00 & 0.02 & 0.08 & 0.00 & 0.03 & 0.02 \\
\hline 2 & 15 & 0.00 & 0.00 & 0.02 & -0.01 & -0.01 & -0.03 & 0.03 & 0.01 & 0.09 \\
\hline 3 & 1 & -0.04 & 0.02 & 0.03 & -0.03 & -0.04 & -0.19 & 0.05 & 0.01 & 0.10 \\
\hline 4 & 6 & 0.00 & 0.00 & 0.01 & -0.03 & -0.03 & -0.12 & 0.00 & -0.02 & 0.01 \\
\hline 5 & 15 & 0.00 & 0.00 & -0.01 & 0.01 & 0.03 & 0.16 & -0.03 & 0.00 & -0.02 \\
\hline 6 & 6 & 0.00 & 0.00 & 0.00 & -0.02 & -0.01 & -0.02 & -0.03 & -0.03 & -0.04 \\
\hline 7 & 1 & -0.07 & 0.00 & -0.03 & 0.00 & 0.04 & 0.19 & -0.06 & -0.01 & -0.09 \\
\hline 8 & 6 & 0.49 & 0.05 & 0.11 & 0.02 & -0.01 & 0.04 & 0.01 & 0.03 & -0.08 \\
\hline 9 & 6 & 0.07 & 0.11 & 0.00 & -0.02 & 0.00 & -0.05 & 0.01 & 0.03 & -0.03 \\
\hline 10 & 6 & 0.06 & 0.16 & 0.07 & -0.03 & 0.03 & -0.01 & -0.02 & 0.03 & -0.06 \\
\hline 11 & 1 & 0.19 & 0.12 & 0.16 & 0.01 & 0.01 & 0.08 & -0.01 & 0.03 & -0.09 \\
\hline
\end{tabular}




$\begin{array}{rrrrrrrrrrr}12 & 1 & 0.09 & 0.13 & -0.05 & -0.05 & 0.03 & -0.08 & -0.02 & 0.03 & -0.01 \\ 13 & 1 & 0.03 & 0.21 & 0.06 & -0.07 & 0.08 & -0.01 & -0.06 & 0.02 & -0.06 \\ 14 & 1 & 0.19 & 0.07 & 0.10 & 0.07 & -0.07 & 0.04 & 0.05 & 0.03 & -0.08 \\ 15 & 1 & 0.08 & 0.03 & -0.01 & 0.02 & -0.06 & -0.06 & 0.05 & 0.04 & -0.03 \\ 16 & 6 & 0.00 & 0.00 & 0.00 & -0.04 & -0.05 & -0.17 & -0.04 & -0.06 & -0.10 \\ 17 & 1 & 0.00 & 0.00 & -0.01 & -0.05 & -0.06 & -0.21 & -0.06 & -0.08 & -0.16 \\ 18 & 6 & 0.00 & 0.00 & 0.00 & -0.05 & -0.07 & -0.26 & -0.01 & -0.06 & -0.05 \\ 19 & 1 & -0.01 & 0.01 & -0.01 & -0.07 & -0.10 & -0.40 & -0.01 & -0.07 & -0.06 \\ 20 & 7 & -0.27 & -0.06 & -0.04 & 0.03 & -0.06 & -0.08 & 0.02 & 0.01 & -0.15 \\ 21 & 1 & -0.31 & -0.08 & -0.05 & 0.04 & -0.01 & 0.00 & 0.03 & -0.13 & -0.39 \\ 22 & 1 & -0.34 & -0.08 & -0.07 & -0.04 & -0.18 & -0.09 & 0.01 & 0.27 & -0.18 \\ 23 & 6 & -0.15 & -0.08 & -0.06 & 0.11 & 0.00 & -0.25 & 0.03 & -0.18 & 0.16 \\ 24 & 1 & -0.16 & -0.22 & -0.14 & 0.12 & -0.03 & -0.32 & 0.03 & -0.20 & 0.12 \\ 25 & 1 & -0.11 & -0.07 & -0.05 & 0.11 & -0.06 & -0.34 & 0.01 & -0.03 & 0.43 \\ 26 & 1 & -0.09 & -0.07 & -0.03 & 0.18 & 0.13 & -0.24 & 0.06 & -0.47 & 0.20\end{array}$

thermodynamics:

Sum of electronic and zero-point Energies=

$-1176.770731$

Sum of electronic and thermal Enthalpies=

$-1176.753780$

Sum of electronic and thermal Free Energies=

$-1176.816888$

Total free energy in solution:

with all non electrostatic terms

$(\mathrm{a} \cdot \mathrm{u})=$.

Optimized geometry, three lower frequencies, thermochemistry and PCM energy for $\mathbf{2 a}$.

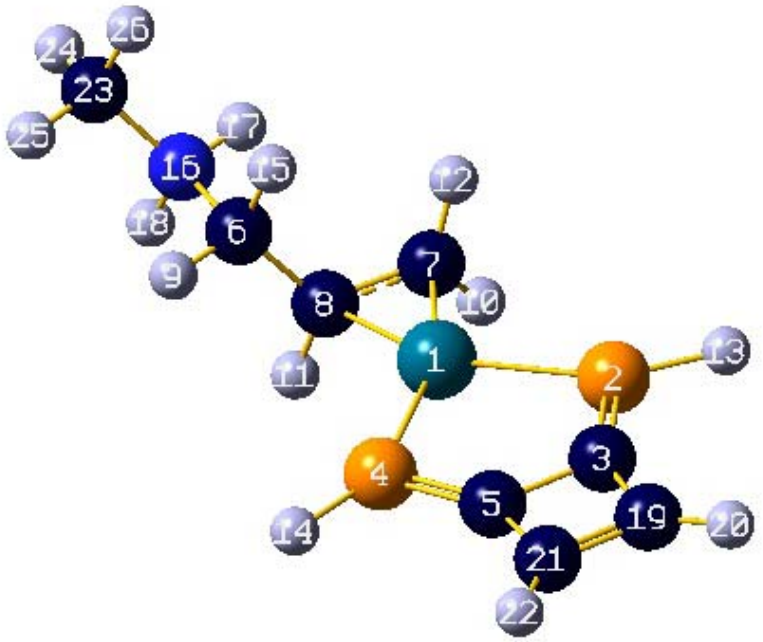

\begin{tabular}{|c|c|c|c|c|c|}
\hline \multirow{2}{*}{$\begin{array}{l}\text { Center } \\
\text { Number }\end{array}$} & \multirow{2}{*}{$\begin{array}{l}\text { Atomic } \\
\text { Number }\end{array}$} & \multirow{2}{*}{$\begin{array}{l}\text { Atomic } \\
\text { Type }\end{array}$} & \multicolumn{3}{|c|}{ Coordinates (Angstroms) } \\
\hline & & & $\mathrm{x}$ & $\mathrm{Y}$ & Z \\
\hline 1 & 46 & 0 & 0.125642 & -0.375878 & -0.240404 \\
\hline 2 & 15 & 0 & -1.884376 & -1.467227 & 0.404462 \\
\hline 3 & 6 & 0 & -3.117923 & -0.331090 & 0.382547 \\
\hline 4 & 15 & 0 & -1.257401 & 1.568836 & -0.381626 \\
\hline 5 & 6 & 0 & -2.821071 & 1.099838 & 0.009059 \\
\hline 6 & 6 & 0 & 2.979404 & 0.555034 & 0.193066 \\
\hline 7 & 6 & 0 & 1.826210 & -1.636458 & -0.341793 \\
\hline 8 & 6 & 0 & 2.206448 & -0.327365 & -0.727368 \\
\hline 9 & 1 & 0 & 2.884799 & 1.620000 & -0.035399 \\
\hline 10 & 1 & 0 & 1.675512 & -2.405089 & -1.096976 \\
\hline 11 & 1 & 0 & 2.299986 & -0.093367 & -1.789640 \\
\hline 12 & 1 & 0 & 2.055893 & -2.013286 & 0.656790 \\
\hline 13 & 1 & 0 & -2.509264 & -2.683973 & 0.780430 \\
\hline 14 & 1 & 0 & -1.368855 & 2.951372 & -0.686295 \\
\hline 15 & 1 & 0 & 2.737503 & 0.388078 & 1.246061 \\
\hline 16 & 7 & 0 & 4.491688 & 0.248808 & 0.076917 \\
\hline
\end{tabular}




$\begin{array}{ll}17 & 1 \\ 18 & 1 \\ 19 & 6 \\ 20 & 1 \\ 21 & 6 \\ 22 & 1 \\ 23 & 6 \\ 24 & 1 \\ 25 & 1 \\ 26 & 1\end{array}$

$\begin{array}{rrr}4.607297 & -0.754160 & 0.257597 \\ 4.762511 & 0.377450 & -0.903488 \\ -4.511880 & 0.067475 & 0.540116 \\ -5.411673 & -0.470618 & 0.817923 \\ -4.240359 & 1.376572 & 0.196212 \\ -4.842559 & 2.273016 & 0.096325 \\ 5.384792 & 1.048342 & 0.965034 \\ 6.423268 & 0.756142 & 0.797142 \\ 5.251462 & 2.106232 & 0.733246 \\ 5.105702 & 0.858705 & 2.002645\end{array}$

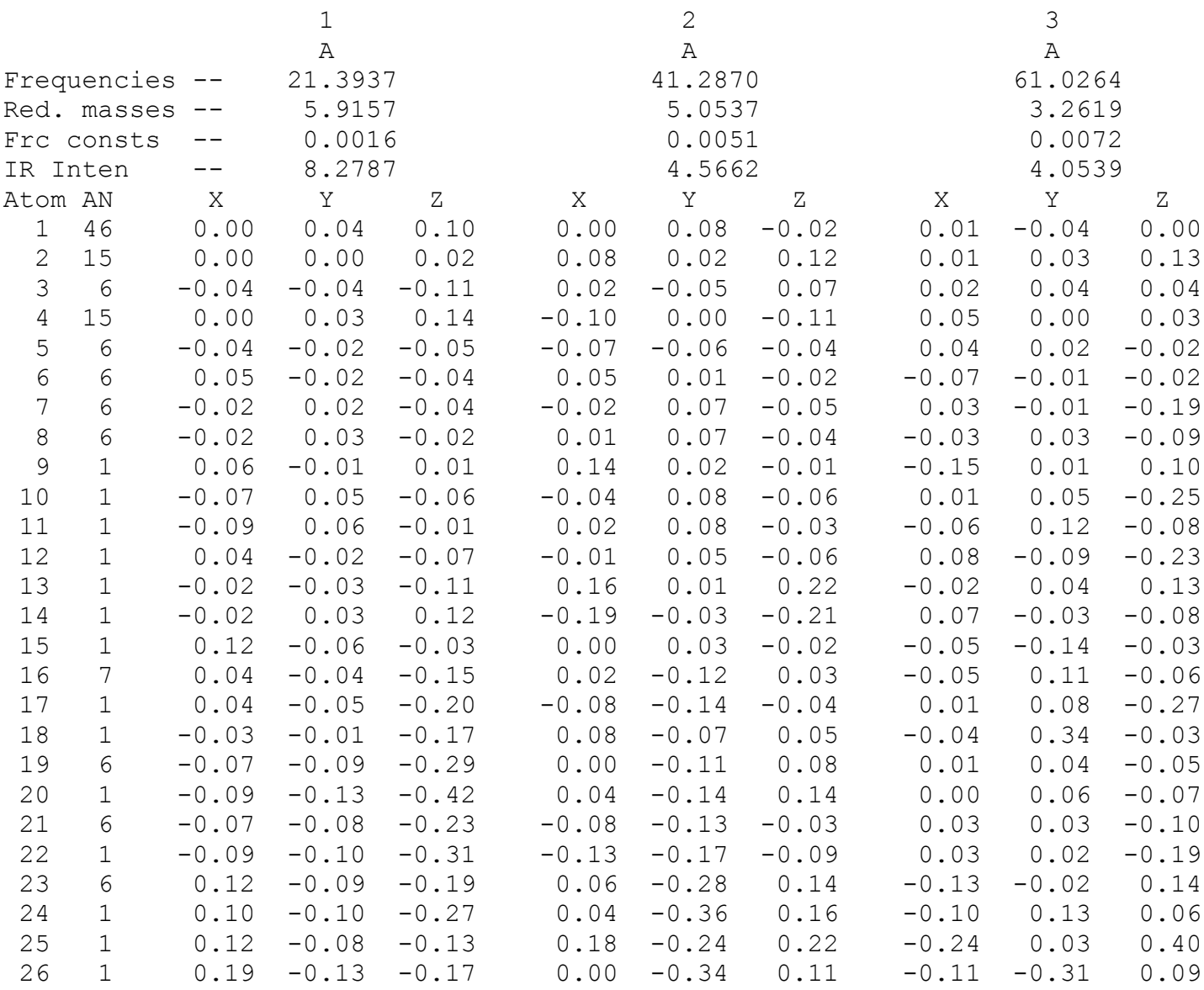

thermodynamics:

Sum of electronic and zero-point Energies= $\quad-1176.783718$

Sum of electronic and thermal Enthalpies= $\quad-1176.767312$

Sum of electronic and thermal Free Energies= $\quad-1176.828515$

Total free energy in solution:

with all non electrostatic terms (a.u.) = -1177.059673

Optimized geometry, three lower frequencies, thermochemistry and PCM energy for $\mathbf{T S}_{\mathbf{2 a}-7 \mathbf{a}}$. 


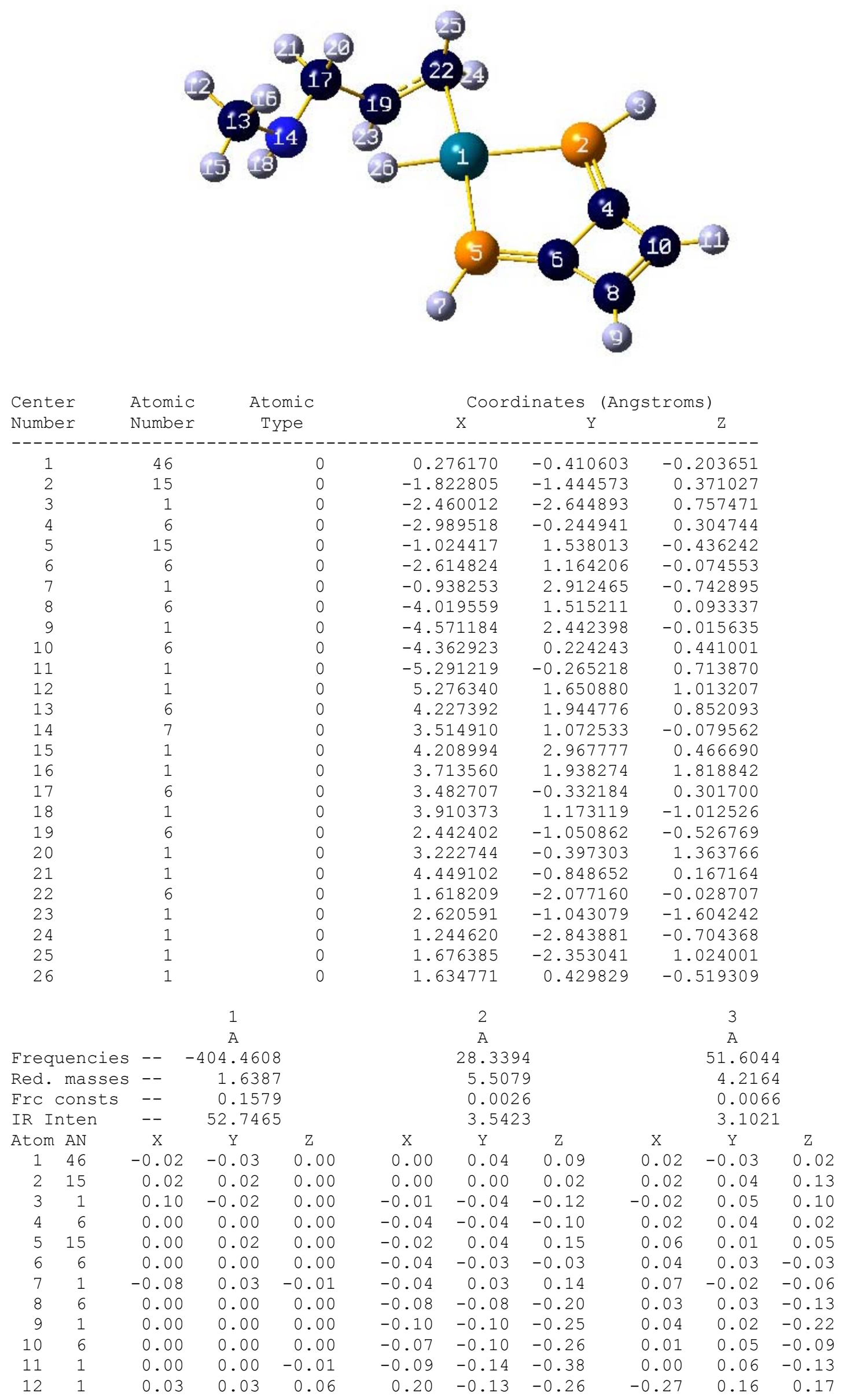




$\begin{array}{rrrrrrrrrrr}13 & 6 & 0.03 & 0.01 & 0.02 & 0.19 & -0.08 & -0.14 & -0.29 & 0.04 & 0.10 \\ 14 & 7 & 0.07 & 0.01 & 0.00 & 0.06 & -0.03 & -0.09 & -0.10 & 0.02 & -0.03 \\ 15 & 1 & 0.02 & 0.01 & 0.02 & 0.19 & -0.07 & -0.11 & -0.39 & 0.06 & 0.18 \\ 16 & 1 & 0.00 & 0.01 & 0.00 & 0.29 & -0.09 & -0.08 & -0.38 & -0.10 & 0.05 \\ 17 & 6 & 0.05 & 0.01 & 0.01 & 0.05 & -0.04 & -0.13 & 0.01 & -0.02 & -0.14 \\ 18 & 1 & 0.14 & -0.01 & 0.03 & -0.03 & -0.02 & -0.13 & -0.05 & 0.14 & 0.01 \\ 19 & 6 & -0.03 & 0.14 & -0.03 & -0.03 & 0.02 & -0.07 & -0.01 & 0.00 & -0.13 \\ 20 & 1 & -0.01 & 0.03 & 0.00 & 0.13 & -0.06 & -0.11 & 0.07 & -0.12 & -0.13 \\ 21 & 1 & 0.02 & -0.04 & 0.05 & 0.02 & -0.06 & -0.21 & 0.03 & 0.05 & -0.23 \\ 22 & 6 & -0.06 & 0.07 & -0.01 & -0.01 & 0.01 & -0.05 & 0.02 & -0.04 & -0.14 \\ 23 & 1 & 0.15 & -0.07 & 0.00 & -0.11 & 0.05 & -0.08 & -0.04 & 0.04 & -0.14 \\ 24 & 1 & 0.07 & 0.01 & -0.01 & -0.07 & 0.04 & -0.05 & -0.01 & -0.01 & -0.16 \\ 25 & 1 & 0.00 & 0.05 & -0.02 & 0.05 & -0.02 & -0.06 & 0.09 & -0.09 & -0.16 \\ 26 & 1 & 0.43 & -0.76 & -0.34 & -0.01 & 0.03 & 0.05 & 0.03 & -0.05 & -0.05\end{array}$

thermodynamics:

Sum of electronic and zero-point Energies=

$-1176.756728$

Sum of electronic and thermal Enthalpies=

$-1176.740789$

Sum of electronic and thermal Free Energies=

$-1176.800349$

Total free energy in solution:

with all non electrostatic terms

$(\mathrm{a} \cdot \mathrm{u})=$.

Optimized geometry, three lower frequencies, thermochemistry and PCM energy for 3a.

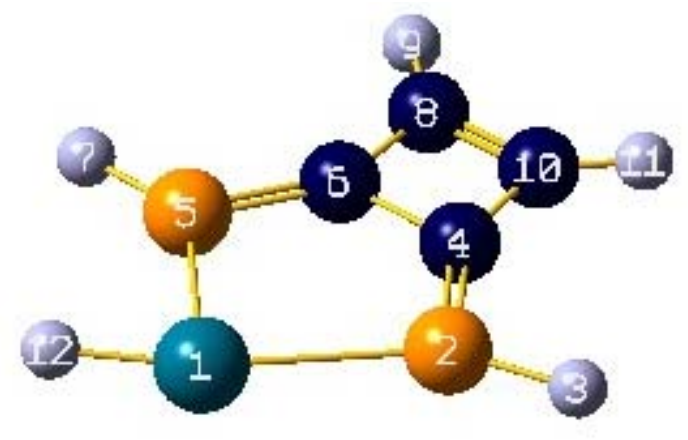

\begin{tabular}{|c|c|c|c|c|c|}
\hline \multirow{2}{*}{$\begin{array}{l}\text { Center } \\
\text { Number }\end{array}$} & \multirow{2}{*}{$\begin{array}{l}\text { Atomic } \\
\text { Number }\end{array}$} & \multirow{2}{*}{$\begin{array}{l}\text { Atomic } \\
\text { Type }\end{array}$} & \multicolumn{3}{|c|}{ Coordinates (Angstroms) } \\
\hline & & & $\mathrm{X}$ & $\mathrm{Y}$ & Z \\
\hline 1 & 46 & 0 & 1.500266 & 0.055851 & -0.020053 \\
\hline 2 & 15 & 0 & -0.380668 & 1.711312 & 0.035933 \\
\hline 3 & 1 & 0 & -0.878954 & 3.033146 & 0.035848 \\
\hline 4 & 6 & 0 & -1.703740 & 0.684351 & 0.010697 \\
\hline 5 & 15 & 0 & -0.042405 & -1.532872 & 0.071661 \\
\hline 6 & 6 & 0 & -1.549207 & -0.812792 & -0.011905 \\
\hline 7 & 1 & 0 & -0.069986 & -2.929268 & -0.073123 \\
\hline 8 & 6 & 0 & -2.999851 & -0.913949 & -0.045886 \\
\hline 9 & 1 & 0 & -3.685765 & -1.752685 & -0.079205 \\
\hline 10 & 6 & 0 & -3.145975 & 0.459044 & -0.027910 \\
\hline 11 & 1 & 0 & -3.999771 & 1.127673 & -0.048756 \\
\hline 12 & 1 & 0 & 2.360973 & -1.224557 & -0.076226 \\
\hline
\end{tabular}

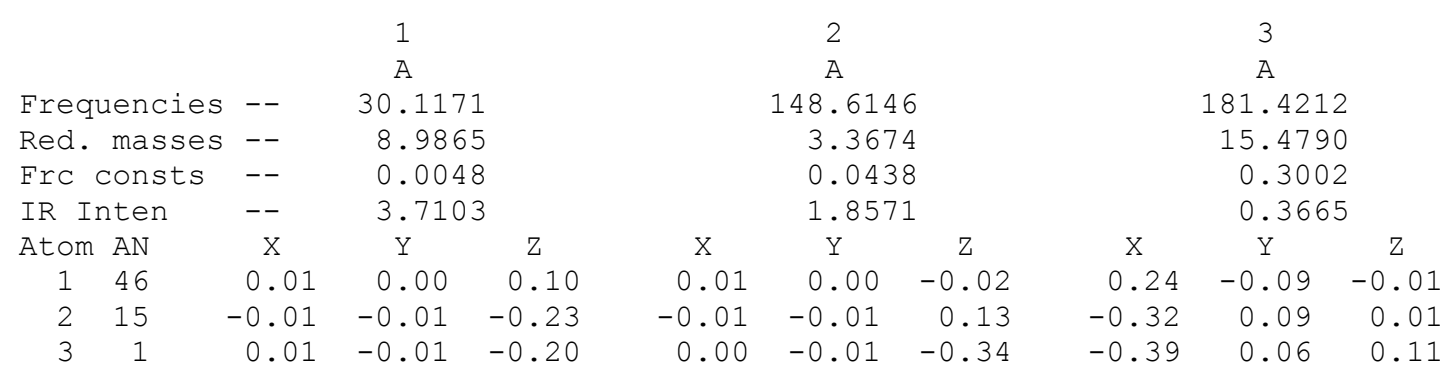




$\begin{array}{rrrrrrrrrrr}4 & 6 & -0.01 & -0.01 & -0.05 & -0.01 & 0.00 & -0.10 & -0.30 & 0.07 & 0.01 \\ 5 & 15 & -0.01 & 0.02 & -0.36 & 0.00 & 0.02 & -0.06 & -0.11 & 0.20 & 0.03 \\ 6 & 6 & -0.02 & -0.01 & 0.07 & -0.02 & -0.01 & 0.21 & -0.15 & 0.11 & -0.05 \\ 7 & 1 & 0.01 & -0.06 & 0.37 & 0.00 & -0.04 & 0.49 & -0.15 & 0.20 & 0.03 \\ 8 & 6 & -0.03 & 0.00 & 0.28 & -0.02 & 0.00 & 0.15 & -0.15 & -0.06 & -0.05 \\ 9 & 1 & -0.05 & 0.00 & 0.48 & -0.03 & 0.00 & 0.33 & -0.06 & -0.14 & -0.08 \\ 10 & 6 & -0.02 & 0.00 & 0.19 & 0.00 & 0.00 & -0.27 & -0.31 & -0.08 & 0.04 \\ 11 & 1 & -0.01 & 0.01 & 0.32 & 0.01 & 0.01 & -0.60 & -0.38 & -0.17 & 0.13 \\ 12 & 1 & 0.04 & 0.01 & 0.41 & 0.02 & 0.01 & -0.01 & 0.23 & -0.04 & 0.02\end{array}$

thermodynamics:

Sum of electronic and zero-point Energies=

$-964.339277$

Sum of electronic and thermal Enthalpies=

$-964.329836$

Sum of electronic and thermal Free Energies=

$-964.374485$

Total free energy in solution:

with all non electrostatic terms

$($ a.u. $)=-964.494732$

Optimized geometry, three lower frequencies, thermochemistry and PCM energy for 3'a.

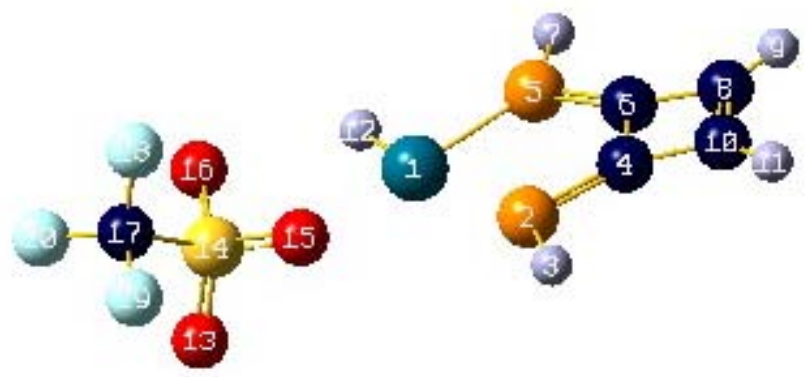

\begin{tabular}{|c|c|c|c|c|c|}
\hline \multirow{2}{*}{$\begin{array}{l}\text { Center } \\
\text { Number }\end{array}$} & \multirow{2}{*}{$\begin{array}{l}\text { Atomic } \\
\text { Number }\end{array}$} & \multirow{2}{*}{$\begin{array}{c}\text { Atomic } \\
\text { Type }\end{array}$} & \multicolumn{3}{|c|}{ Coordinates (Angstroms) } \\
\hline & & & $\mathrm{X}$ & $Y$ & Z \\
\hline 1 & 46 & 0 & 0.633309 & -0.435495 & 0.023057 \\
\hline 2 & 15 & 0 & 2.060103 & 1.659584 & 0.285591 \\
\hline 3 & 1 & 0 & 2.226480 & 3.048103 & 0.523410 \\
\hline 4 & 6 & 0 & 3.617061 & 1.076069 & 0.069777 \\
\hline 5 & 15 & 0 & 2.588706 & -1.446739 & -0.302241 \\
\hline 6 & 6 & 0 & 3.873063 & -0.381984 & -0.207284 \\
\hline 7 & 1 & 0 & 3.064392 & -2.746636 & -0.564218 \\
\hline 8 & 6 & 0 & 5.296711 & -0.063357 & -0.251212 \\
\hline 9 & 1 & 0 & 6.185702 & -0.661006 & -0.419350 \\
\hline 10 & 6 & 0 & 5.066176 & 1.270646 & 0.002313 \\
\hline 11 & 1 & 0 & 5.703022 & 2.141203 & 0.113190 \\
\hline 12 & 1 & 0 & -0.005904 & -1.814061 & -0.178714 \\
\hline 13 & 8 & 0 & -2.980339 & -0.411552 & 1.886658 \\
\hline 14 & 16 & 0 & -2.494295 & -0.444417 & 0.511790 \\
\hline 15 & 8 & 0 & -1.227943 & 0.405904 & 0.313044 \\
\hline 16 & 8 & 0 & -2.427274 & -1.728105 & -0.191477 \\
\hline 17 & 6 & 0 & -3.695301 & 0.593180 & -0.472866 \\
\hline 18 & 9 & 0 & -3.301214 & 0.694899 & -1.748172 \\
\hline 19 & 9 & 0 & -3.793527 & 1.824381 & 0.040439 \\
\hline 20 & 9 & 0 & -4.904274 & 0.023593 & -0.446045 \\
\hline
\end{tabular}

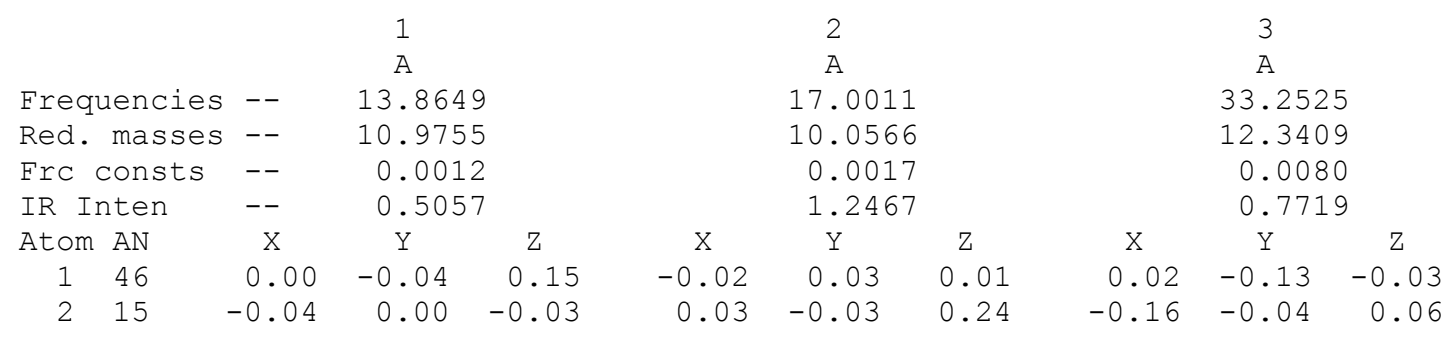




$\begin{array}{rrrrrrrrrrr}3 & 1 & -0.07 & 0.03 & -0.14 & 0.08 & -0.07 & 0.41 & -0.29 & -0.03 & 0.11 \\ 4 & 6 & -0.04 & 0.05 & -0.16 & 0.01 & -0.03 & 0.07 & -0.11 & 0.10 & 0.05 \\ 5 & 15 & 0.01 & -0.01 & 0.08 & -0.05 & 0.04 & -0.22 & 0.10 & 0.02 & -0.05 \\ 6 & 6 & -0.02 & 0.04 & -0.11 & -0.03 & 0.00 & -0.15 & 0.01 & 0.13 & 0.00 \\ 7 & 1 & 0.02 & 0.00 & 0.07 & -0.09 & 0.07 & -0.43 & 0.21 & 0.07 & -0.09 \\ 8 & 6 & -0.04 & 0.09 & -0.28 & -0.03 & -0.02 & -0.19 & -0.02 & 0.24 & 0.01 \\ 9 & 1 & -0.03 & 0.11 & -0.35 & -0.05 & -0.01 & -0.33 & 0.03 & 0.32 & 0.00 \\ 10 & 6 & -0.06 & 0.10 & -0.33 & 0.01 & -0.05 & 0.01 & -0.12 & 0.22 & 0.06 \\ 11 & 1 & -0.08 & 0.13 & -0.46 & 0.03 & -0.07 & 0.09 & -0.19 & 0.26 & 0.10 \\ 12 & 1 & 0.02 & -0.06 & 0.20 & -0.05 & 0.07 & -0.14 & 0.09 & -0.16 & -0.06 \\ 13 & 8 & -0.17 & -0.16 & -0.07 & -0.13 & 0.15 & 0.07 & -0.01 & 0.09 & 0.02 \\ 14 & 16 & -0.04 & -0.05 & -0.03 & -0.02 & 0.04 & 0.11 & -0.03 & -0.02 & 0.02 \\ 15 & 8 & 0.00 & -0.06 & 0.16 & 0.00 & 0.02 & 0.16 & 0.04 & -0.13 & -0.02 \\ 16 & 8 & 0.00 & 0.01 & -0.13 & 0.05 & -0.02 & 0.23 & -0.16 & -0.05 & 0.07 \\ 17 & 6 & 0.08 & 0.06 & -0.05 & 0.06 & -0.06 & -0.08 & 0.07 & 0.08 & 0.00 \\ 18 & 9 & 0.21 & 0.18 & 0.00 & 0.18 & -0.16 & -0.05 & -0.01 & -0.13 & -0.04 \\ 19 & 9 & 0.05 & 0.02 & 0.05 & -0.01 & -0.02 & -0.19 & 0.33 & 0.16 & -0.13 \\ 20 & 9 & 0.06 & 0.09 & -0.23 & 0.06 & -0.08 & -0.15 & -0.03 & 0.31 & 0.16\end{array}$

thermodynamics:

Sum of electronic and zero-point Energies=

$-1925.760521$

Sum of electronic and thermal Enthalpies=

$-1925.742333$

Sum of electronic and thermal Free Energies=

$-1925.810131$

Total free energy in solution:

with all non electrostatic terms

$(\mathrm{a} \cdot \mathrm{u} \cdot)=-1925.886806$

Optimized geometry, three lower frequencies, thermochemistry and PCM energy for $\mathbf{4 a}$.

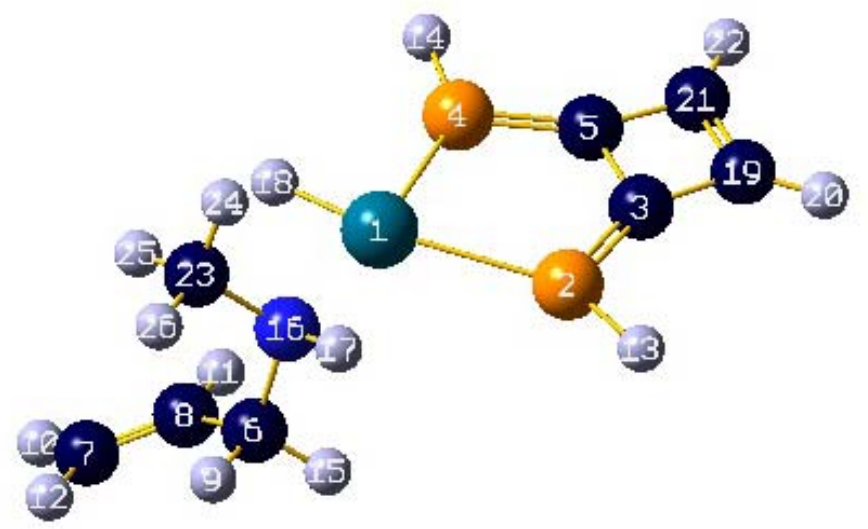

\begin{tabular}{cccrrr}
$\begin{array}{c}\text { Center } \\
\text { Number }\end{array}$ & $\begin{array}{c}\text { Atomic } \\
\text { Number }\end{array}$ & $\begin{array}{c}\text { Atomic } \\
\text { Type }\end{array}$ & \multicolumn{3}{c}{ Coordinates (Angstroms) } \\
----1 & $\mathrm{X}$ & $\mathrm{Z}$ \\
1 & 46 & 0 & 0.293559 & -0.219297 & 0.355469 \\
2 & 15 & 0 & -1.356933 & 1.610994 & -0.165557 \\
3 & 6 & 0 & -2.813994 & 0.799001 & -0.331421 \\
4 & 15 & 0 & -1.527640 & -1.568829 & 0.199611 \\
5 & 6 & 0 & -2.903270 & -0.693052 & -0.161595 \\
6 & 6 & 0 & 2.913939 & 0.925713 & -0.721693 \\
7 & 6 & 0 & 4.572789 & -0.778294 & -1.490786 \\
8 & 6 & 0 & 3.308095 & -0.406880 & -1.279575 \\
9 & 1 & 0 & 3.805498 & 1.544469 & -0.547218 \\
10 & 1 & 0 & 4.813622 & -1.738966 & -1.937861 \\
11 & 1 & 0 & 2.490765 & -1.073865 & -1.556383 \\
12 & 1 & 0 & 5.413179 & -0.132944 & -1.240650 \\
13 & 1 & 0 & -1.646159 & 2.978629 & -0.389327 \\
14 & 1 & 0 & -1.819797 & -2.940304 & 0.315199 \\
15 & 1 & 0 & 2.267145 & 1.458811 & -1.429489
\end{tabular}




$\begin{array}{ll}16 & 7 \\ 17 & 1 \\ 18 & 1 \\ 19 & 6 \\ 20 & 1 \\ 21 & 6 \\ 22 & 1 \\ 23 & 6 \\ 24 & 1 \\ 25 & 1 \\ 26 & 1\end{array}$

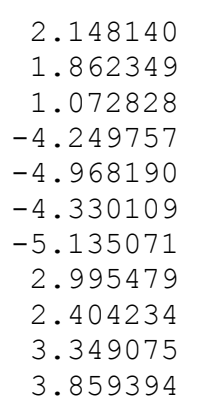

0.824103
1.774979
-1.517748
0.788465
1.567561
-0.580038
-1.304215
0.345105
0.324268
-0.662149
1.007819

0.561418

0.797064

0.642505

$-0.593488$

$-0.822877$

$-0.436883$

$-0.493575$

1. 681332

2.598895

1. 458779

1.817263

\begin{tabular}{|c|c|c|c|c|c|c|c|c|c|c|}
\hline & \multicolumn{3}{|c|}{1} & \multicolumn{3}{|c|}{2} & \multicolumn{3}{|c|}{3} \\
\hline & & \multicolumn{3}{|c|}{ A } & \multicolumn{3}{|c|}{ A } & \multicolumn{3}{|c|}{ A } \\
\hline \multicolumn{2}{|c|}{ Frequencies } & -- & \multicolumn{2}{|c|}{14.7383} & \multicolumn{3}{|c|}{37.1040} & \multicolumn{3}{|c|}{52.6649} \\
\hline Red. & masses & -- & \multicolumn{2}{|c|}{5.1644} & \multicolumn{3}{|c|}{5.7305} & \multicolumn{3}{|c|}{3.4566} \\
\hline Frc c & consts & -- & \multicolumn{2}{|c|}{0.0007} & \multicolumn{3}{|c|}{0.0046} & \multicolumn{3}{|c|}{0.0056} \\
\hline IR In & iten & -- & \multicolumn{2}{|c|}{0.3155} & \multicolumn{3}{|c|}{2.1103} & \multicolumn{3}{|c|}{1.6284} \\
\hline Atom & $\mathrm{AN}$ & X & $\mathrm{Y}$ & $\mathrm{Z}$ & $\mathrm{X}$ & $\mathrm{Y}$ & Z & $\mathrm{X}$ & $\mathrm{Y}$ & $\mathrm{Z}$ \\
\hline 1 & 46 & 0.00 & -0.02 & 0.08 & 0.01 & 0.05 & 0.05 & -0.02 & -0.03 & 0.02 \\
\hline 2 & 15 & 0.06 & -0.02 & -0.10 & -0.06 & 0.03 & 0.22 & 0.00 & 0.01 & 0.09 \\
\hline 3 & 6 & 0.05 & 0.00 & -0.12 & 0.00 & -0.02 & 0.01 & 0.00 & 0.03 & 0.00 \\
\hline 4 & 15 & -0.02 & 0.01 & 0.10 & 0.05 & 0.00 & -0.01 & -0.06 & 0.01 & 0.06 \\
\hline 5 & 6 & 0.01 & 0.01 & -0.03 & 0.05 & -0.04 & -0.11 & -0.03 & 0.03 & -0.01 \\
\hline 6 & 6 & -0.08 & 0.07 & 0.04 & -0.01 & -0.05 & -0.09 & -0.04 & -0.06 & -0.11 \\
\hline 7 & 6 & -0.14 & 0.13 & -0.24 & -0.15 & -0.21 & -0.03 & 0.21 & 0.17 & -0.05 \\
\hline 8 & 6 & -0.12 & 0.11 & -0.10 & -0.12 & -0.09 & -0.07 & 0.16 & -0.06 & 0.04 \\
\hline 9 & 1 & -0.06 & 0.05 & 0.02 & 0.03 & -0.10 & -0.13 & -0.13 & 0.09 & -0.18 \\
\hline 10 & 1 & -0.17 & 0.17 & -0.33 & -0.22 & -0.24 & -0.01 & 0.36 & 0.16 & 0.05 \\
\hline 11 & 1 & -0.14 & 0.13 & -0.07 & -0.17 & -0.01 & -0.09 & 0.26 & -0.26 & 0.21 \\
\hline 12 & 1 & -0.12 & 0.12 & -0.27 & -0.09 & -0.29 & -0.01 & 0.11 & 0.37 & -0.22 \\
\hline 13 & 1 & 0.10 & -0.03 & -0.20 & -0.09 & 0.03 & 0.24 & 0.03 & 0.01 & 0.05 \\
\hline 14 & 1 & -0.05 & 0.02 & 0.17 & 0.11 & -0.03 & -0.20 & -0.07 & 0.01 & -0.01 \\
\hline 15 & 1 & -0.12 & 0.13 & 0.12 & 0.00 & -0.02 & -0.08 & -0.13 & -0.24 & -0.16 \\
\hline 16 & 7 & 0.01 & -0.04 & 0.08 & 0.02 & 0.04 & -0.06 & -0.01 & -0.03 & -0.09 \\
\hline 17 & 1 & 0.02 & -0.06 & 0.17 & 0.06 & 0.06 & -0.10 & -0.03 & -0.02 & -0.13 \\
\hline 18 & 1 & -0.04 & -0.02 & 0.19 & 0.06 & 0.04 & -0.10 & -0.02 & -0.04 & -0.03 \\
\hline 19 & 6 & 0.06 & 0.01 & -0.19 & 0.03 & -0.07 & -0.15 & 0.01 & 0.04 & -0.08 \\
\hline 20 & 1 & 0.09 & 0.01 & -0.27 & 0.02 & -0.09 & -0.19 & 0.04 & 0.05 & -0.13 \\
\hline 21 & 6 & 0.03 & 0.02 & -0.10 & 0.07 & -0.09 & -0.26 & -0.01 & 0.04 & -0.10 \\
\hline 22 & 1 & 0.02 & 0.03 & -0.10 & 0.11 & -0.12 & -0.41 & -0.01 & 0.05 & -0.15 \\
\hline 23 & 6 & 0.09 & -0.12 & -0.02 & 0.05 & 0.07 & -0.06 & 0.02 & 0.05 & -0.08 \\
\hline 24 & 1 & 0.15 & -0.19 & 0.02 & 0.07 & 0.12 & -0.04 & 0.05 & 0.12 & -0.06 \\
\hline 25 & 1 & 0.07 & -0.10 & -0.12 & 0.02 & 0.06 & -0.03 & 0.01 & 0.03 & -0.02 \\
\hline 26 & 1 & 0.09 & -0.12 & -0.02 & 0.06 & 0.06 & -0.11 & 0.03 & 0.06 & -0.15 \\
\hline
\end{tabular}

thermodynamics:

Sum of electronic and zero-point Energies=

$-1176.762059$

Sum of electronic and thermal Enthalpies=

$-1176.745448$

Sum of electronic and thermal Free Energies=

$-1176.807347$

Total free energy in solution:

with all non electrostatic terms

$(\mathrm{a} \cdot \mathrm{u} \cdot)=-1177.020258$

Optimized geometry, three lower frequencies, thermochemistry and PCM energy for 5a. 


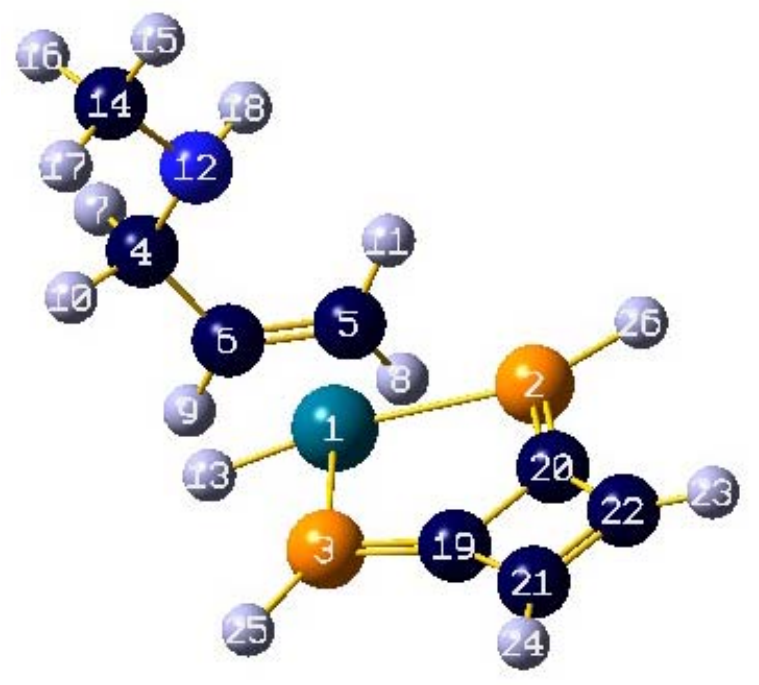

\begin{tabular}{|c|c|c|c|c|c|}
\hline \multirow{2}{*}{$\begin{array}{l}\text { Center } \\
\text { Number }\end{array}$} & \multirow{2}{*}{$\begin{array}{l}\text { Atomic } \\
\text { Number }\end{array}$} & \multirow{2}{*}{$\begin{array}{l}\text { Atomic } \\
\text { Type }\end{array}$} & \multicolumn{3}{|c|}{ Coordinates (Angstroms) } \\
\hline & & & $\mathrm{X}$ & $Y$ & Z \\
\hline 1 & 46 & 0 & .320631 & -.177443 & -.611883 \\
\hline 2 & 15 & 0 & -1.273313 & -1.359709 & .827571 \\
\hline 3 & 15 & 0 & -1.412093 & 1.320067 & -.892572 \\
\hline 4 & 6 & 0 & 3.446306 & .324457 & -.150468 \\
\hline 5 & 6 & 0 & 1.914846 & -1.699451 & -.469791 \\
\hline 6 & 6 & 0 & 2.615588 & -.619597 & -.980257 \\
\hline 7 & 1 & 0 & 4.502287 & .027177 & -.314960 \\
\hline 8 & 1 & 0 & 1.581713 & -2.494077 & -1.13486 \\
\hline 9 & 1 & 0 & 2.802426 & -.591536 & -2.05355 \\
\hline 10 & 1 & 0 & 3.362245 & 1.336087 & -.56238 \\
\hline 11 & 1 & 0 & 1.961357 & -1.934685 & .590388 \\
\hline 12 & 7 & 0 & 3.059929 & .360502 & 1.245037 \\
\hline 13 & 1 & 0 & 1.104738 & .706384 & -1.619197 \\
\hline 14 & 6 & 0 & 3.580025 & 1.524252 & 1.96165 \\
\hline 15 & 1 & 0 & 3.285572 & 1.455700 & 3.012641 \\
\hline 16 & 1 & 0 & 4.676695 & 1.625033 & 1.913126 \\
\hline 17 & 1 & 0 & 3.132665 & 2.434659 & 1.548812 \\
\hline 18 & 1 & 0 & 3.379892 & -.482292 & 1.716211 \\
\hline 19 & 6 & 0 & -2.758990 & .795504 & -.052826 \\
\hline 20 & 6 & 0 & -2.688551 & -.467275 & .76205 \\
\hline 21 & 6 & 0 & -4.147934 & .921783 & .371189 \\
\hline 22 & 6 & 0 & -4.084347 & -.235366 & 1.119828 \\
\hline 23 & 1 & 0 & -4.792074 & -.777738 & 1.736847 \\
\hline 24 & 1 & 0 & -4.923610 & 1.650863 & .165548 \\
\hline 25 & 1 & 0 & -1.705380 & 2.497130 & -1.607648 \\
\hline 26 & 1 & 0 & -1.517620 & -2.485047 & 1.647109 \\
\hline
\end{tabular}

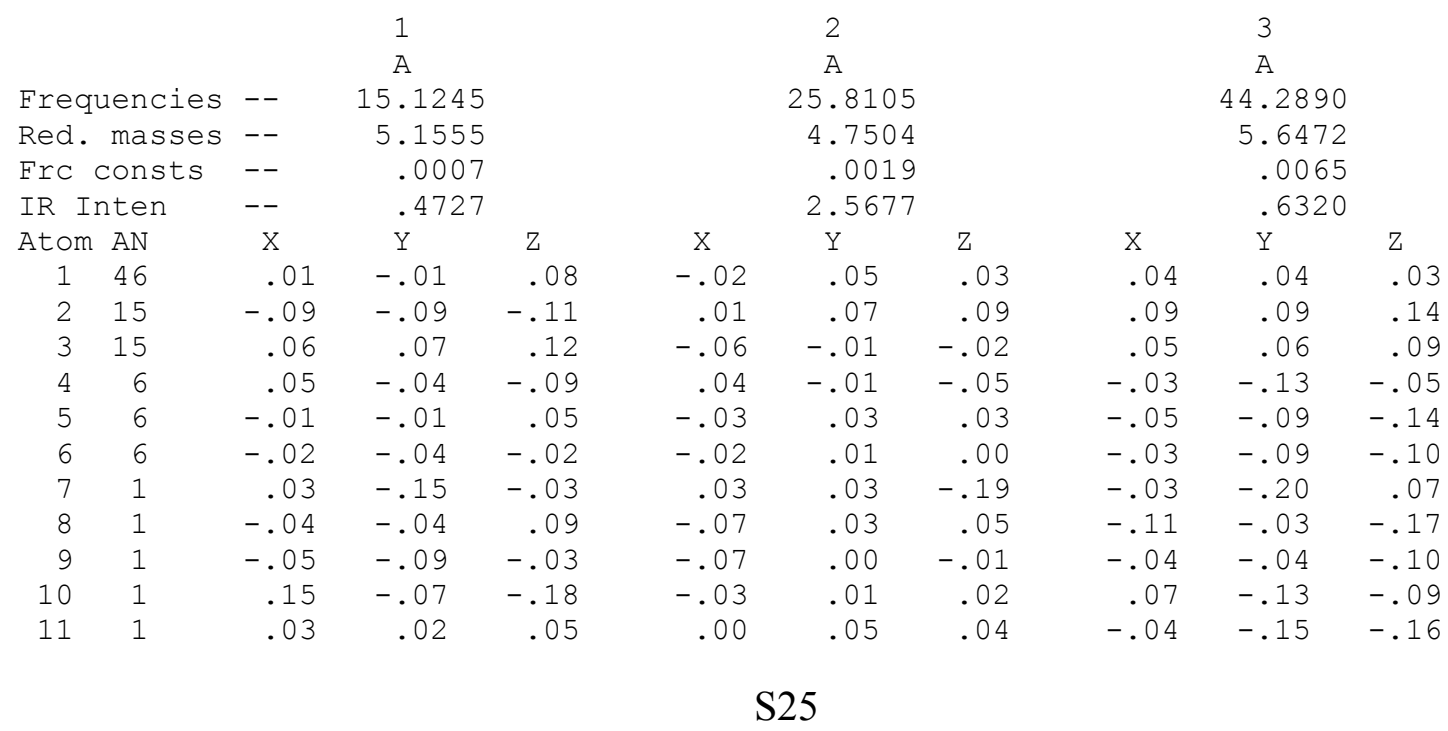




\begin{tabular}{|c|c|c|c|c|c|c|c|c|c|c|}
\hline 12 & 7 & .02 & .13 & -.10 & .21 & -.09 & .00 & -.18 & -.06 & -.09 \\
\hline 13 & 1 & .11 & .02 & .19 & -.06 & .03 & -.01 & -.05 & -.03 & -.09 \\
\hline 14 & 6 & .11 & .14 & -.20 & .28 & -.13 & .00 & -.25 & -.03 & -.09 \\
\hline 15 & 1 & .08 & .27 & -.20 & .40 & -.19 & .03 & -.37 & .02 & -.12 \\
\hline 16 & 1 & .12 & .03 & -.18 & .27 & -.11 & -.12 & -.24 & -.05 & .03 \\
\hline 17 & 1 & .21 & .15 & -.29 & .22 & -.11 & .11 & -.19 & -.05 & -.18 \\
\hline 18 & 1 & -.08 & .14 & -.02 & .28 & -.12 & -.09 & -.24 & -.04 & -.02 \\
\hline 19 & 6 & .00 & .03 & .01 & -.07 & -.06 & -.08 & .01 & -.02 & -.02 \\
\hline 20 & 6 & -.07 & -.05 & -.10 & -.04 & -.02 & -.02 & .04 & .00 & .01 \\
\hline 21 & 6 & -.01 & .02 & -.05 & -.10 & -.13 & -.16 & -.03 & -.12 & -.14 \\
\hline 22 & 6 & -.08 & -.05 & -.15 & -.07 & -.09 & -.11 & -.01 & -.10 & -.12 \\
\hline 23 & 1 & -.13 & -.09 & -.24 & -.07 & -.11 & -.13 & -.03 & -.15 & -.18 \\
\hline 24 & 1 & .01 & .06 & -.02 & -.14 & -.19 & -.24 & -.07 & -.18 & -.24 \\
\hline 25 & 1 & .12 & .14 & .22 & -.10 & -.08 & -.12 & .00 & -.02 & -.0 \\
\hline 26 & 1 & -.16 & -.16 & -.23 & .03 & .07 & .10 & .07 & .06 & .0 \\
\hline
\end{tabular}

thermodynamics:

Sum of electronic and zero-point Energies=

$-1176.751545$

Sum of electronic and thermal Enthalpies=

$-1176.734733$

Sum of electronic and thermal Free Energies=

$-1176.797509$

Total free energy in solution:

with all non electrostatic terms

$(\mathrm{a} \cdot \mathrm{u} \cdot)=-1177.008911$

Optimized geometry, three lower frequencies, thermochemistry and PCM energy for 5'a.

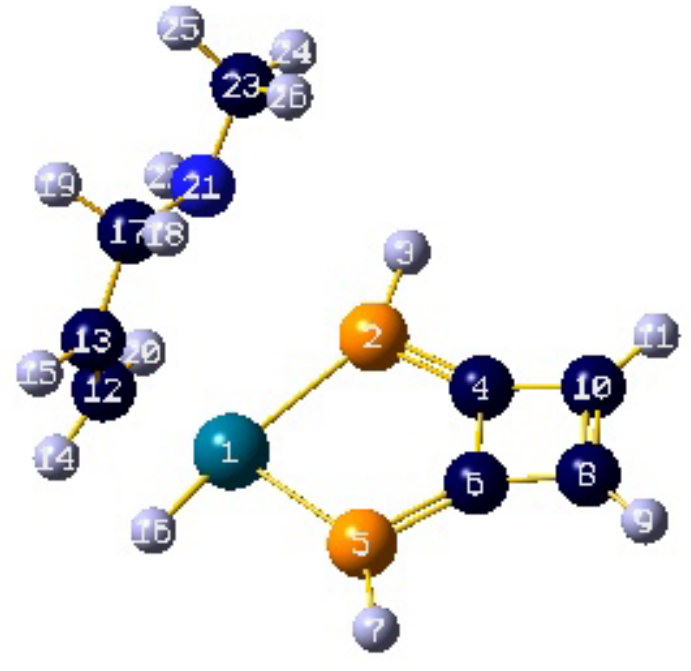

\begin{tabular}{|c|c|c|c|c|c|}
\hline \multirow{2}{*}{$\begin{array}{l}\text { Center } \\
\text { Number } \\
-------\end{array}$} & \multirow{2}{*}{$\begin{array}{l}\text { Atomic } \\
\text { Number }\end{array}$} & \multirow{2}{*}{$\begin{array}{l}\text { Atomic } \\
\text { Type }\end{array}$} & \multicolumn{3}{|c|}{ Coordinates (Angstroms) } \\
\hline & & & $\mathrm{x}$ & Y & Z \\
\hline 1 & 46 & 0 & .273397 & -1.138400 & .102621 \\
\hline 2 & 15 & 0 & -.339647 & 1.172595 & .746925 \\
\hline 3 & 1 & 0 & .018750 & 2.399967 & 1.340776 \\
\hline 4 & 6 & 0 & -1.963640 & 1.303250 & .337114 \\
\hline 5 & 15 & 0 & -1.940437 & -1.323170 & -.533930 \\
\hline 6 & 6 & 0 & -2.706506 & .144493 & -.282130 \\
\hline 7 & 1 & 0 & -2.819862 & -2.247441 & -1.129539 \\
\hline 8 & 6 & 0 & -3.882732 & .996601 & -.344025 \\
\hline 9 & 1 & 0 & -4.900166 & .835561 & -.681716 \\
\hline 10 & 6 & 0 & -3.202352 & 2.064212 & .207755 \\
\hline 11 & 1 & 0 & -3.476171 & 3.082846 & .461069 \\
\hline 12 & 6 & 0 & 2.291413 & -1.216417 & 1.026713 \\
\hline 13 & 6 & 0 & 2.504127 & -.905048 & -.300780 \\
\hline 14 & 1 & 0 & 2.383571 & -2.244083 & 1.369432 \\
\hline 15 & 1 & 0 & 2.723576 & -1.720739 & -.989476 \\
\hline
\end{tabular}




$\begin{array}{ll}16 & 1 \\ 17 & 6 \\ 18 & 1 \\ 19 & 1 \\ 20 & 1 \\ 21 & 7 \\ 22 & 1 \\ 23 & 6 \\ 24 & 1 \\ 25 & 1 \\ 26 & 1\end{array}$

.465664
2.768957
2.182469
3.827174
2.281212
2.468953
3.086743
2.626237
2.445520
3.625759
1.885037
$-2.595063$
.461530
.582823
.453295
$-.449301$
1.571661
1.538388
2.872434
3.668191
3.023374
2.969220

Frequencies -- 20

Red. masses --

Frc consts --

IR Inten --

Atom AN $\mathrm{X}$

$\begin{array}{lll}1 & 46 & .01\end{array}$

$\begin{array}{lll}2 & 15 & .04\end{array}$

$\begin{array}{lll}3 & 1 & .04\end{array}$

$\begin{array}{lll}4 & 6 & .08\end{array}$

$\begin{array}{lll}5 & 15 & .01\end{array}$

$\begin{array}{lll}6 & 6 & .08\end{array}$

$\begin{array}{lll}7 & 1 & .04\end{array}$

$\begin{array}{lll}8 & 6 & .13\end{array}$

$\begin{array}{lll}9 & 1 & .15\end{array}$

$\begin{array}{lll}10 & 6 & .13\end{array}$

$\begin{array}{lll}11 & 1 & .17\end{array}$

$\begin{array}{lll}12 & 6 & .06\end{array}$

$136-.03$

141.11

$\begin{array}{lll}15 & 1 & -.03\end{array}$

$\begin{array}{lll}16 & 1 & .02\end{array}$

$\begin{array}{lll}17 & 6 & -.12\end{array}$

$\begin{array}{lll}18 & 1 & -.14\end{array}$

$19-1 \quad-.12$

$\begin{array}{lll}20 & 1 & .08\end{array}$

$217-.16$

$\begin{array}{lll}22 & 1 & -.15\end{array}$

$236-.26$

$\begin{array}{lll}24 & 1 & -.29\end{array}$

$25 \quad 1 \quad-.28$

$\begin{array}{lll}26 & 1 & -.29\end{array}$
1

20.0979

5.2395

.0012

5.5136

$\mathrm{Y}$

$-.04$

$-.07$

$-.11$

.02

.02

.07

.10

.13

.19

.09

.10

$-.01$

.03

$-.01$

.05

.00

.05

.02

.11

$-.03$

.03

.06

.04

.03

.10

$-.01$

\section{2}

43.2280

4. 6028

.0051

.6781

$\mathrm{Y}$

.04

.15

.23

.02

$-.01$

$-.08$

$-.17$

$-.18$

$-.29$

$-.10$

$-.12$

$-.06$

$-.07$

$-.09$

$-.09$

$-.08$

$-.06$

$-.05$

$-.08$

$-.04$

$-.05$

$-.07$

$-.05$

$-.05$

$-.08$

$-.03$

thermodynamics:

Sum of electronic and zero-point Energies=

Sum of electronic and thermal Enthalpies=

Sum of electronic and thermal Free Energies= $x$
-.01
.01

.01

.01

.01

$-.06$

$-.01$

.02

.02

.04

.07

.06

.09

$-.09$

$-.03$

$-.05$

$-.11$

$-.08$

.10

.03

.03

.10

.14

.11

.11
$-.01$

.02

.08

.01

$-.08$

$-.02$

$-.08$

.01

.01

.04

.07

$-.06$

.05

$-.09$

.11

$-.02$

.09

.19

.10

$-.13$

.02

$-.11$

.08

.01

.07

.21
$-.380793$

$-.871534$

$-1.791983$

$-1.202189$

1.797353

.014627

.823936

$-.640509$

.087885

$-1.077137$

$-1.441135$
3

A

70.2370

5.2836

.0154

5.6685

Z

.00

$-.05$

$-.17$

$-.08$

.19

.02

.19

$-.09$

$-.10$

$-.18$

$-.29$

$-.15$

$-.14$

$-.24$

$-.23$

.02

$-.04$

.01

$-.11$

$-.09$

.08

.07

.22

.31

.24
.23

$\mathrm{X}$

.00

$-.13$

$-.12$

$-.09$

.02

$-.03$

.10

$-.02$

.02

$-.09$

$-.11$

.05

$-.01$

.03

$-.07$

.02

$-.01$

$-.13$

$-.05$

.11

.18

.27

.25

.42

.21

.17

$-1176.754710$

$-1176.738271$

$-1176.798972$

Total free energy in solution:

with all non electrostatic terms

$(\mathrm{a} \cdot \mathrm{u} \cdot)=-1177.009930$

Optimized geometry, three lower frequencies, thermochemistry and PCM energy for $\mathbf{T S}_{\mathbf{5 a}}$, a. 


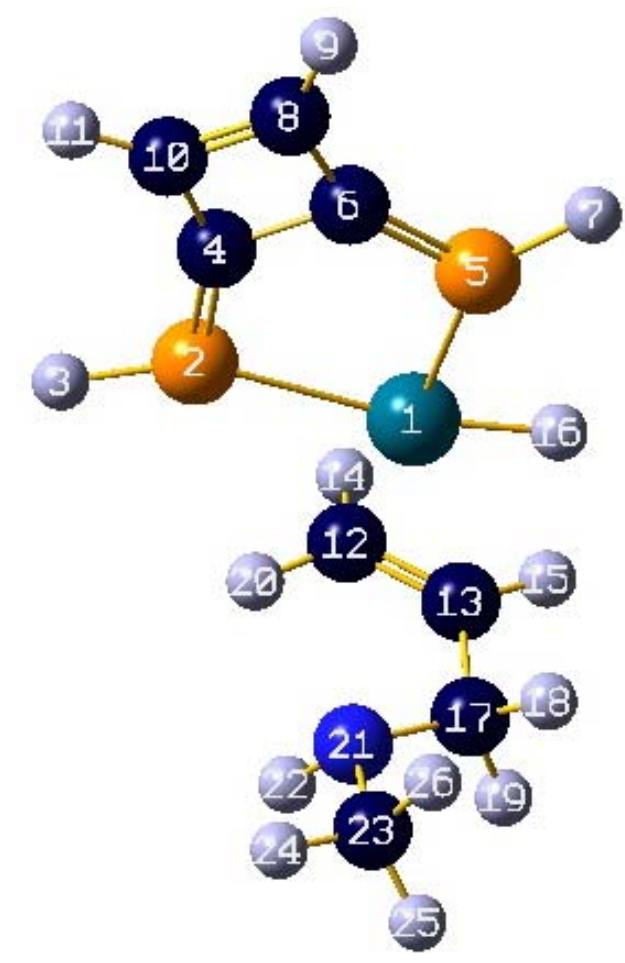

\begin{tabular}{cccrrr} 
Center & Atomic & Atomic & \multicolumn{2}{c}{ Coordinates (Angstroms) } \\
Number & Number & Type & \multicolumn{2}{c}{ Y } & Z \\
-1 & 46 & 0 & .310921 & -.758568 & .184393 \\
1 & 15 & 0 & -1.044408 & 1.042780 & 1.191589 \\
2 & 1 & 0 & -1.121034 & 2.087186 & 2.141483 \\
3 & 6 & 0 & -2.528266 & .962353 & .420327 \\
4 & 15 & 0 & -1.563644 & -1.117423 & -1.101982 \\
5 & 6 & 0 & -2.780522 & -.062728 & -.652436 \\
6 & 1 & 0 & -2.018693 & -2.009160 & -2.091627 \\
7 & 6 & 0 & -4.131177 & .471373 & -.765609 \\
8 & 1 & 0 & -4.982456 & .212857 & -1.385209 \\
9 & 6 & 0 & -3.900056 & 1.415650 & .213809 \\
10 & 1 & 0 & -4.499741 & 2.196157 & .668895 \\
11 & 6 & 0 & 2.004728 & -.553960 & 1.617723 \\
12 & 6 & 0 & 2.646132 & -.963597 & .466664 \\
13 & 1 & 0 & 1.754102 & -1.275869 & 2.392929 \\
14 & 1 & 0 & 2.882242 & -2.021219 & .354690 \\
15 & 1 & 0 & .897057 & -1.972663 & -.576489 \\
16 & 6 & 0 & 3.329279 & -.044232 & -.509762 \\
17 & 1 & 0 & 3.056910 & -.340604 & -1.530057 \\
18 & 1 & 0 & 4.414809 & -.257165 & -.427881 \\
19 & 1 & 0 & 2.000502 & .496093 & 1.895514 \\
20 & 7 & 0 & 2.992095 & 1.352545 & -.325478 \\
21 & 1 & 0 & 3.475897 & 1.716081 & .492570 \\
22 & 6 & 0 & 3.329291 & 2.186525 & -1.478315 \\
23 & 1 & 0 & 3.096571 & 3.229292 & -1.244609 \\
24 & 1 & 0 & 4.388397 & 2.125885 & -1.778538 \\
25 & 1 & 0 & 2.712716 & 1.890773 & -2.333911 \\
26 & & 0 & & &
\end{tabular}

\begin{tabular}{|c|c|c|c|c|c|c|c|c|c|}
\hline & & 1 & & & 2 & & & 3 & \\
\hline & & A & & & A & & & A & \\
\hline Frequencies & -- & -20.0745 & & & 24.5582 & & & 46.6395 & \\
\hline Red. masses & -- & 4.9970 & & & 4.7326 & & & 5.2599 & \\
\hline Frc consts & -- & .0012 & & & .0017 & & & .0067 & \\
\hline IR Inten & -- & .4720 & & & 2.0892 & & & 1.0724 & \\
\hline Atom AN & X & Y & $\mathrm{Z}$ & $\mathrm{X}$ & $\mathrm{Y}$ & z & X & Y & Z \\
\hline 146 & .01 & .07 & .00 & .02 & -.03 & .04 & -.03 & -.03 & .04 \\
\hline 215 & -.10 & -.09 & .12 & -.01 & -.08 & .10 & -.08 & -.12 & .12 \\
\hline
\end{tabular}




\begin{tabular}{|c|c|c|c|c|c|c|c|c|c|c|}
\hline 3 & 1 & -.17 & -.18 & .21 & -.02 & -.10 & .12 & -.07 & -.08 & .08 \\
\hline 4 & 6 & -.08 & -.08 & .07 & .04 & .02 & -.01 & -.03 & -.02 & .00 \\
\hline 5 & 15 & .05 & .09 & -.08 & .05 & .04 & -.03 & -.04 & -.08 & .07 \\
\hline 6 & 6 & .00 & .00 & -.03 & .07 & .08 & -.07 & -.01 & .01 & -.03 \\
\hline 7 & 1 & .12 & .16 & -.18 & .09 & .12 & -.12 & .01 & .02 & -.04 \\
\hline 8 & 6 & -.02 & -.05 & -.01 & .10 & .15 & -.14 & .04 & .11 & -.15 \\
\hline 9 & 1 & .00 & -.03 & -.06 & .14 & .22 & -.22 & .08 & .19 & -.24 \\
\hline 10 & 6 & -.09 & -.12 & .08 & .08 & .09 & -.09 & .03 & .09 & -.12 \\
\hline 11 & 1 & -.14 & -.19 & .13 & .08 & .10 & -.10 & .05 & .14 & -.18 \\
\hline 12 & 6 & -.03 & .08 & .02 & .03 & -.03 & .03 & .06 & .11 & -.09 \\
\hline 13 & 6 & .00 & -.02 & .07 & .02 & .00 & .01 & .01 & .08 & -.11 \\
\hline 14 & 1 & -.07 & .13 & .06 & .07 & -.05 & .02 & .11 & .13 & -.05 \\
\hline 15 & 1 & -.03 & -.04 & .15 & .05 & .01 & .00 & .02 & .08 & -.09 \\
\hline 16 & 1 & .10 & .17 & -.10 & .05 & .02 & -.01 & .03 & .05 & -.05 \\
\hline 17 & 6 & .07 & -.10 & .04 & -.05 & .03 & -.01 & -.01 & .05 & -.15 \\
\hline 18 & 1 & .15 & -.19 & .04 & .01 & -.05 & .00 & -.15 & .12 & -.13 \\
\hline 19 & 1 & .06 & -.09 & .15 & -.03 & .14 & .03 & -.02 & -.06 & -.27 \\
\hline 20 & 1 & .01 & .09 & -.04 & .01 & -.04 & .04 & .05 & .12 & -.13 \\
\hline 21 & 7 & .05 & -.09 & -.10 & -.20 & .00 & -.08 & .15 & .08 & -.06 \\
\hline 22 & 1 & -.02 & -.01 & -.10 & -.23 & .10 & -.10 & .21 & -.03 & -.04 \\
\hline 23 & 6 & .13 & -.18 & -.15 & -.29 & -.02 & -.12 & .23 & .11 & -.01 \\
\hline 24 & 1 & .09 & -.17 & -.26 & -.41 & -.04 & -.18 & .37 & .12 & .06 \\
\hline 25 & 1 & .15 & -.19 & -.06 & -.29 & .08 & -.12 & .22 & -.01 & -.04 \\
\hline 26 & 1 & .20 & -.27 & -.17 & -.26 & -.14 & -.11 & .18 & .24 & -.02 \\
\hline
\end{tabular}

thermodynamics:

Sum of electronic and zero-point Energies=

$-1176.751343$

Sum of electronic and thermal Enthalpies=

$-1176.735477$

Sum of electronic and thermal Free Energies=

$-1176.794805$

Total free energy in solution:

with all non electrostatic terms (a.u.) $=-1177.007554$

Optimized geometry, three lower frequencies, thermochemistry and PCM energy for $\mathbf{6 a}$.

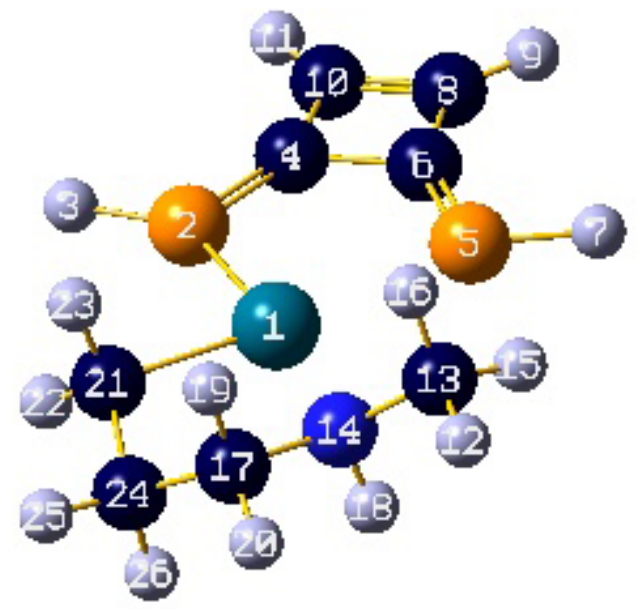

\begin{tabular}{|c|c|c|c|c|c|}
\hline \multirow{2}{*}{$\begin{array}{l}\text { Center } \\
\text { Number }\end{array}$} & \multirow{2}{*}{$\begin{array}{l}\text { Atomic } \\
\text { Number }\end{array}$} & \multirow{2}{*}{$\begin{array}{c}\text { Atomic } \\
\text { Type }\end{array}$} & \multicolumn{3}{|c|}{ Coordinates (Angstroms) } \\
\hline & & & $\mathrm{X}$ & $Y$ & Z \\
\hline 1 & 46 & 0 & .523886 & -.151218 & -.014462 \\
\hline 2 & 15 & 0 & -1.290019 & -1.537404 & .149743 \\
\hline 3 & 1 & 0 & -1.541202 & -2.918070 & .290238 \\
\hline 4 & 6 & 0 & -2.728506 & -.693371 & .074431 \\
\hline 5 & 15 & 0 & -1.256532 & 1.638103 & -.177149 \\
\hline 6 & 6 & 0 & -2.705489 & .801605 & -.079982 \\
\hline 7 & 1 & 0 & -1.618924 & 3.001368 & -.315145 \\
\hline
\end{tabular}




$\begin{array}{rl}8 & 6 \\ 9 & 1 \\ 10 & 6 \\ 11 & 1 \\ 12 & 1 \\ 13 & 6 \\ 14 & 7 \\ 15 & 1 \\ 16 & 1 \\ 17 & 6 \\ 18 & 1 \\ 19 & 1 \\ 20 & 1 \\ 21 & 6 \\ 22 & 1 \\ 23 & 1 \\ 24 & 6 \\ 25 & 1 \\ 26 & 1\end{array}$

-4.165493
-4.931696
-4.184567
-4.969496
3.435212
2.454576
2.351699
1.676637
2.319557
3.488717
2.383200
3.582136
4.423745
1.803681
1.405387
1.860783
3.137359
3.920792
3.089812

3.089812

$$
\begin{array}{rr}
.758868 & -.064963 \\
1.522677 & -.138598 \\
-.612951 & .076756 \\
-1.356421 & .159051 \\
2.719732 & .315554 \\
2.258432 & .088538 \\
.962235 & -.212019 \\
2.934537 & .127944 \\
2.101403 & 1.561011 \\
.051473 & .101952 \\
1.146824 & -1.217024 \\
.015361 & 1.193230 \\
.458221 & -.305569 \\
-1.752483 & .140752 \\
-2.646312 & -.347392 \\
-1.922176 & 1.224001 \\
-1.314932 & -.446182 \\
-2.044643 & -.191705 \\
-1.282445 & -1.542948
\end{array}
$$

\begin{tabular}{rrr}
\multicolumn{4}{l}{} \\
\multicolumn{4}{l}{ Frequencies } & \multicolumn{1}{l}{--} \\
Red. masses & \multicolumn{1}{l}{--} \\
Frc consts & \multicolumn{1}{l}{--} \\
IR Inten & \multicolumn{1}{c}{--} \\
Atom AN & X \\
1 & 46 & .00 \\
2 & 15 & .00 \\
3 & 1 & .01 \\
4 & 6 & .00 \\
5 & 15 & -.01 \\
6 & 6 & -.01 \\
7 & 1 & -.02 \\
8 & 6 & -.01 \\
9 & 1 & -.01 \\
10 & 6 & .00 \\
11 & 1 & .00 \\
12 & 1 & .06 \\
13 & 6 & .08 \\
14 & 7 & -.01 \\
15 & 1 & .04 \\
16 & 1 & .21 \\
17 & 6 & .02 \\
18 & 1 & -.11 \\
19 & 1 & .14 \\
20 & 1 & -.02 \\
21 & 6 & .00 \\
22 & 1 & -.06 \\
23 & 1 & .11 \\
24 & 6 & -.06 \\
25 & 1 & -.04 \\
26 & 1 & -.17 \\
& &
\end{tabular}

\begin{tabular}{cc}
1 & \\
A & \\
33.3786 & \\
5.4847 & \\
.0036 & \\
3.7083 & \\
$Y$ & $Z$ \\
.01 & .09 \\
.01 & .12 \\
.00 & .01 \\
-.01 & -.07 \\
.01 & .13 \\
-.01 & -.06 \\
.00 & .05 \\
-.04 & -.05 \\
-.05 & -.037 \\
-.04 & -.26 \\
-.06 & -.37 \\
-.02 & -.19 \\
-.01 & -.08 \\
.00 & -.08 \\
.00 & .02 \\
-.02 & -.06 \\
-.02 & -.01 \\
.01 & -.08 \\
-.03 & -.22 \\
-.03 & -.32 \\
.00 & -.04 \\
.00 & .01 \\
.01 & -.04 \\
-.01 & -.018 \\
-.02 & -.06 \\
-.01 & -.17 \\
& \\
\hline
\end{tabular}

2

50.3866

3.8096

.0057

.2878

X

.00

$-.01$

$-.01$

.00

.00

.00

.01

.00

.00

.00

$-.01$

.02

.01

.00

.03

$-.02$

$-.01$

.02

$-.09$

.01

$-.01$

.02

$-.08$

.03

.02

.11

$$
\mathrm{Y}
$$

$$
.00
$$

$-.01$

$-.03$

$-.01$

.01

.01

.02

.01

.02

.00

$-.01$

.04

.08

$-.03$

.04

.26

.01

$-.18$

.09

$-.02$

$-.01$

$-.02$

.02

$-.03$

$-.01$

$-.10$
3

A

85.1584

3.9537

.0169

1.1118

$\mathrm{X}$

.00

Y Z

$-.05-.03$

$-.01 \quad .14$

$.00 \quad .10$

$.04 \quad .04$
-.01

$-.01 \quad .07$

$.04 \quad .00$

$-.01-.04$

$.08-.10$

$.09-.19$

$.08-.04$

$.11-.06$

$.08 \quad .04$

$.06-.05$

$.02 \quad .00$

$.01-.16$

$.10-.05$

$.08 \quad .12$

$-.02-.01$

$.04 \quad .13$

$.15 \quad .26$

$-.04-.19$

$.05-.39$

$-.24-.22$

$.09 \quad .00$

$.12 \quad .03$

$.16-.01$

thermodynamics:

Sum of electronic and zero-point Energies=

$-1176.802332$

Sum of electronic and thermal Enthalpies=

$-1176.786776$

Sum of electronic and thermal Free Energies=

$-1176.844855$

Total free energy in solution:

with all non electrostatic terms

$(\mathrm{a} . \mathrm{u})=$.

Optimized geometry, three lower frequencies, thermochemistry and PCM energy for 7a. 


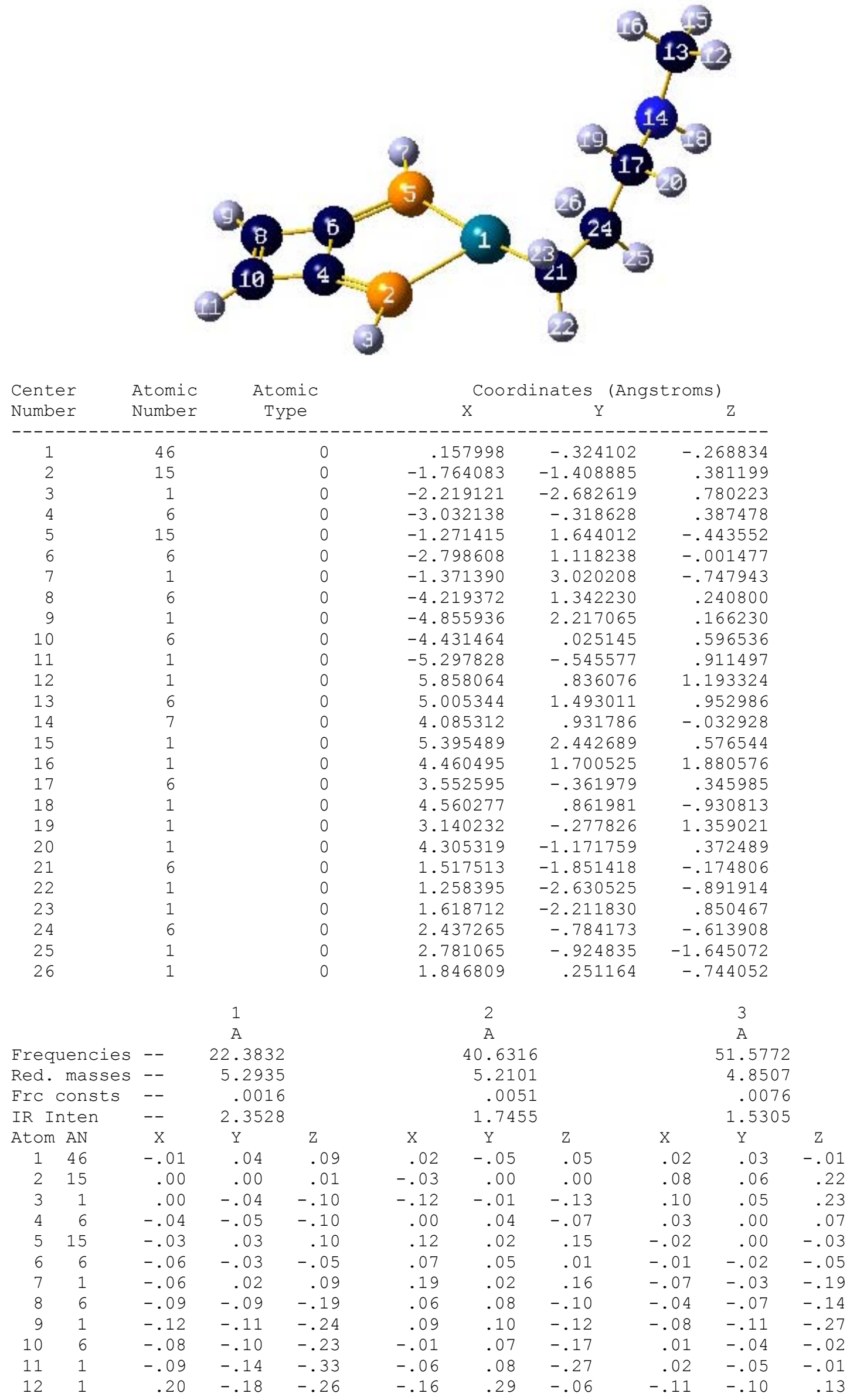




$\begin{array}{rrrrrrrrrrr}13 & 6 & .22 & -.13 & -.16 & -.24 & .18 & -.07 & -.12 & -.10 & .17 \\ 14 & 7 & .09 & -.04 & -.09 & -.16 & .07 & -.08 & -.05 & -.02 & .06 \\ 15 & 1 & .23 & -.13 & -.17 & -.36 & .23 & -.06 & -.13 & -.04 & .30 \\ 16 & 1 & .32 & -.13 & -.10 & -.28 & .11 & -.08 & -.17 & -.23 & .17 \\ 17 & 6 & .06 & -.03 & -.08 & -.01 & .00 & -.10 & -.02 & -.08 & -.11 \\ 18 & 1 & .01 & -.04 & -.14 & -.14 & .13 & -.08 & -.02 & .11 & .07 \\ 19 & 1 & .14 & -.03 & -.05 & .03 & -.08 & -.08 & -.02 & -.22 & -.10 \\ 20 & 1 & .03 & -.06 & -.15 & .07 & .07 & -.17 & .01 & -.06 & -.21 \\ 21 & 6 & -.01 & .04 & .01 & .01 & -.07 & -.05 & -.02 & -.01 & -.19 \\ 22 & 1 & -.04 & .05 & .01 & -.02 & -.06 & -.06 & -.10 & .06 & -.25 \\ 23 & 1 & .03 & .02 & .00 & .05 & -.09 & -.06 & .03 & -.12 & -.24 \\ 24 & 6 & -.02 & .04 & -.02 & -.02 & -.06 & -.07 & -.01 & -.01 & -.15 \\ 25 & 1 & -.09 & .07 & -.04 & -.05 & -.03 & -.08 & -.01 & .04 & -.16 \\ 26 & 1 & .00 & .06 & .07 & -.04 & -.06 & -.05 & .00 & .00 & -.11\end{array}$

thermodynamics:

Sum of electronic and zero-point Energies=

$-1176.763212$

Sum of electronic and thermal Enthalpies=

$-1176.746919$

Sum of electronic and thermal Free Energies=

$-1176.807952$

Total free energy in solution:

with all non electrostatic terms

$(\mathrm{a} \cdot \mathrm{u} \cdot)=-1177.026430$

Optimized geometry, three lower frequencies, thermochemistry and PCM energy for $\mathbf{T S}_{7 \mathbf{a}-5 \mathbf{a}}$.

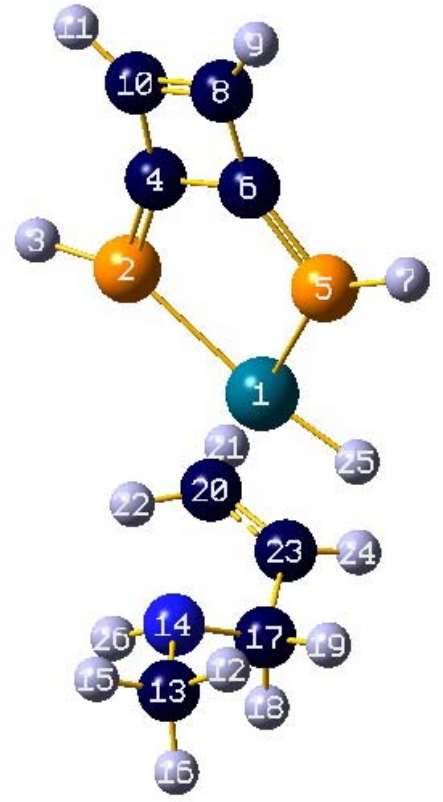

\begin{tabular}{|c|c|c|c|c|c|}
\hline \multirow{2}{*}{$\begin{array}{l}\text { Center } \\
\text { Number }\end{array}$} & \multirow{2}{*}{$\begin{array}{l}\text { Atomic } \\
\text { Number }\end{array}$} & \multirow{2}{*}{$\begin{array}{c}\text { Atomic } \\
\text { Type }\end{array}$} & \multicolumn{3}{|c|}{ Coordinates (Angstroms) } \\
\hline & & & $\mathrm{X}$ & $\mathrm{Y}$ & Z \\
\hline 1 & 46 & 0 & 0.321953 & -0.243636 & -0.544223 \\
\hline 2 & 15 & 0 & -1.345721 & -1.328109 & 0.863484 \\
\hline 3 & 1 & 0 & -1.634646 & -2.425721 & 1.705168 \\
\hline 4 & 6 & 0 & -2.735853 & -0.404233 & 0.733503 \\
\hline 5 & 15 & 0 & -1.364558 & 1.300534 & -0.932547 \\
\hline 6 & 6 & 0 & -2.748308 & 0.837193 & -0.116727 \\
\hline 7 & 1 & 0 & -1.612616 & 2.470095 & -1.677774 \\
\hline 8 & 6 & 0 & -4.144722 & 1.011334 & 0.264645 \\
\hline 9 & 1 & 0 & -4.894323 & 1.755051 & 0.018514 \\
\hline 10 & 6 & 0 & -4.134021 & -0.126565 & 1.044852 \\
\hline 11 & 1 & 0 & -4.873083 & -0.632796 & 1.655763 \\
\hline
\end{tabular}




$\begin{array}{ll}12 & 1 \\ 13 & 6 \\ 14 & 7 \\ 15 & 1 \\ 16 & 1 \\ 17 & 6 \\ 18 & 1 \\ 19 & 1 \\ 20 & 6 \\ 21 & 1 \\ 22 & 1 \\ 23 & 6 \\ 24 & 1 \\ 25 & 1 \\ 26 & 1\end{array}$

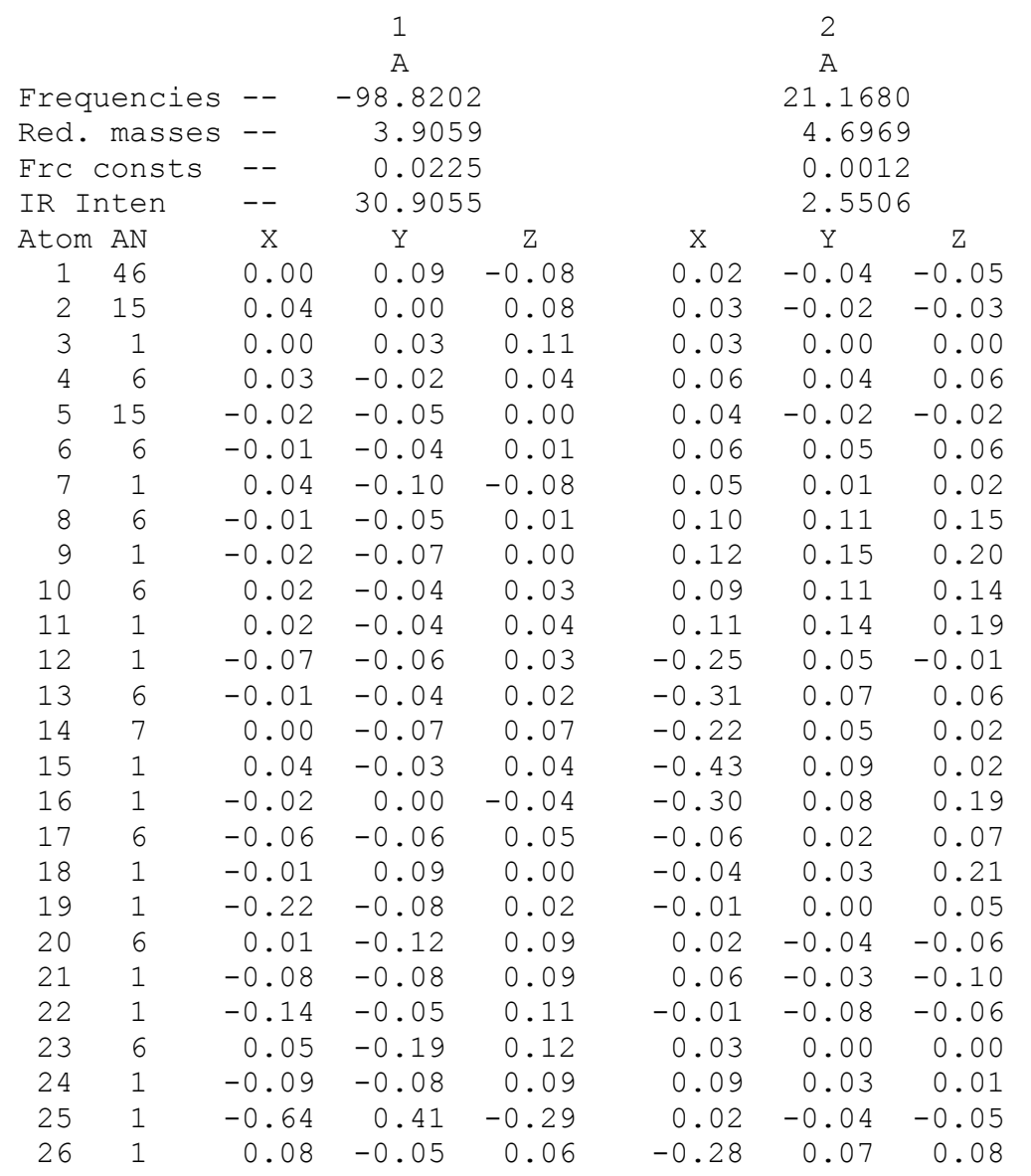

$\begin{array}{rrr}3.261842 & 2.577277 & 1.223629 \\ 3.649693 & 1.700252 & 1.752543 \\ 3.074971 & 0.489189 & 1.168203 \\ 3.334512 & 1.775127 & 2.797032 \\ 4.750925 & 1.733849 & 1.717618 \\ 3.478174 & 0.264294 & -0.204448 \\ 4.514762 & -0.115475 & -0.310267 \\ 3.462734 & 1.223054 & -0.733632 \\ 1.891843 & -1.743117 & -0.305503 \\ 1.551869 & -2.598706 & -0.886206 \\ 1.968730 & -1.884612 & 0.769749 \\ 2.600244 & -0.722454 & -0.933044 \\ 2.783636 & -0.815522 & -2.003579 \\ 1.227258 & 0.542105 & -1.542426 \\ 3.335659 & -0.307383 & 1.744274\end{array}$

\begin{tabular}{rrr} 
& \multicolumn{3}{c}{ I } \\
& A \\
& 37.9808 \\
\multicolumn{3}{c}{4.7568} \\
\multicolumn{3}{c}{0.0040} \\
X & 0.7511 \\
0.03 & 0.03 & \multicolumn{1}{c}{0.05} \\
0.02 & 0.00 & 0.02 \\
-0.03 & -0.07 & -0.09 \\
0.00 & -0.04 & -0.04 \\
0.07 & 0.08 & 0.14 \\
0.03 & 0.00 & 0.01 \\
0.09 & 0.08 & 0.13 \\
-0.01 & -0.06 & -0.09 \\
-0.02 & -0.07 & -0.12 \\
-0.04 & -0.10 & -0.15 \\
-0.07 & -0.16 & -0.24 \\
0.00 & 0.03 & -0.34 \\
-0.14 & 0.06 & -0.18 \\
-0.16 & 0.04 & -0.12 \\
-0.27 & 0.21 & -0.23 \\
-0.13 & -0.03 & -0.05 \\
-0.01 & -0.14 & -0.05 \\
-0.03 & -0.23 & 0.11 \\
0.12 & -0.19 & -0.15 \\
-0.05 & -0.10 & -0.04 \\
-0.12 & -0.07 & -0.05 \\
-0.04 & -0.13 & -0.05 \\
-0.02 & -0.13 & -0.04 \\
-0.07 & -0.11 & -0.05 \\
-0.06 & 0.06 & -0.01 \\
-0.30 & 0.09 & 0.00 \\
& &
\end{tabular}

thermodynamics:

Sum of electronic and zero-point Energies=

$-1176.751980$

Sum of electronic and thermal Enthalpies=

$-1176.735810$

Sum of electronic and thermal Free Energies=

$-1176.796556$

Total free energy in solution:

with all non electrostatic terms

$(\mathrm{a} \cdot \mathrm{u} \cdot)=-1177.008920$

Optimized geometry, three lower frequencies, thermochemistry and PCM energy for $\mathbf{8 a}$. 


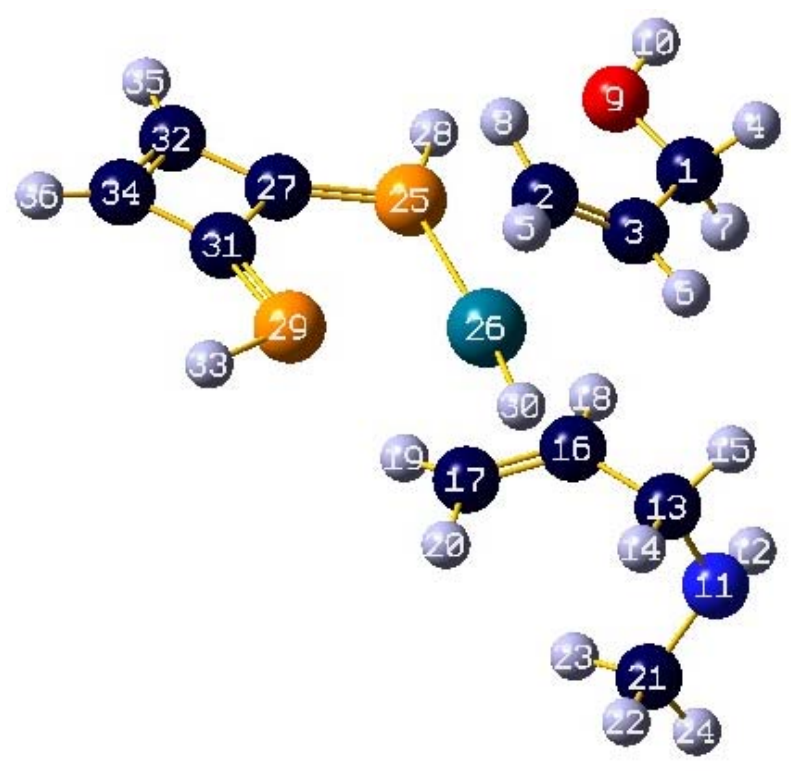

\begin{tabular}{|c|c|c|c|c|c|}
\hline \multirow{2}{*}{$\begin{array}{l}\text { Center } \\
\text { Number }\end{array}$} & \multirow{2}{*}{$\begin{array}{l}\text { Atomic } \\
\text { Number }\end{array}$} & \multirow{2}{*}{$\begin{array}{c}\text { Atomic } \\
\text { Type }\end{array}$} & \multicolumn{3}{|c|}{ Coordinates (Angstroms) } \\
\hline & & & $\mathrm{X}$ & $Y$ & Z \\
\hline 1 & 6 & 0 & -0.954653 & 3.080875 & 0.285987 \\
\hline 2 & 6 & 0 & 0.400273 & 2.008039 & -1.594533 \\
\hline 3 & 6 & 0 & -0.803831 & 2.268496 & -0.969029 \\
\hline 4 & 1 & 0 & -1.220027 & 4.102552 & -0.032030 \\
\hline 5 & 1 & 0 & 0.419690 & 1.729496 & -2.644290 \\
\hline 6 & 1 & 0 & -1.727202 & 2.157662 & -1.536527 \\
\hline 7 & 1 & 0 & -1.793296 & 2.703205 & 0.888676 \\
\hline 8 & 1 & 0 & 1.332062 & 2.349930 & -1.153471 \\
\hline 9 & 8 & 0 & 0.248398 & 3.087064 & 1.032479 \\
\hline 10 & 1 & 0 & 0.263946 & 3.861600 & 1.612547 \\
\hline 11 & 7 & 0 & -4.516384 & -0.842012 & 0.606979 \\
\hline 12 & 1 & 0 & -4.669353 & -0.360069 & 1.487349 \\
\hline 13 & 6 & 0 & -3.371420 & -0.322759 & -0.114470 \\
\hline 14 & 1 & 0 & -3.359214 & -0.772323 & -1.116157 \\
\hline 15 & 1 & 0 & -3.514347 & 0.755960 & -0.243369 \\
\hline 16 & 6 & 0 & -2.062330 & -0.609081 & 0.597393 \\
\hline 17 & 6 & 0 & -1.261167 & -1.703644 & 0.325107 \\
\hline 18 & 1 & 0 & -1.938801 & -0.114614 & 1.563691 \\
\hline 19 & 1 & 0 & -0.565041 & -2.093464 & 1.063476 \\
\hline 20 & 1 & 0 & -1.502798 & -2.364317 & -0.505147 \\
\hline 21 & 6 & 0 & -4.610985 & -2.285273 & 0.746733 \\
\hline 22 & 1 & 0 & -4.654114 & -2.740025 & -0.249987 \\
\hline 23 & 1 & 0 & -3.783097 & -2.756508 & 1.303801 \\
\hline 24 & 1 & 0 & -5.547092 & -2.527183 & 1.258348 \\
\hline 25 & 15 & 0 & 1.389162 & 0.432309 & 1.372294 \\
\hline 26 & 46 & 0 & -0.176614 & 0.087317 & -0.555025 \\
\hline 27 & 6 & 0 & 2.790521 & -0.425694 & 1.054839 \\
\hline 28 & 1 & 0 & 1.576954 & 1.069238 & 2.617876 \\
\hline 29 & 15 & 0 & 1.798750 & -1.302305 & -1.396375 \\
\hline 30 & 1 & 0 & -1.011599 & -0.200318 & -1.802501 \\
\hline 31 & 6 & 0 & 2.972925 & -1.215568 & -0.203382 \\
\hline 32 & 6 & 0 & 4.150731 & -0.832714 & 1.433466 \\
\hline 33 & 1 & 0 & 2.364773 & -2.132583 & -2.397699 \\
\hline 34 & 6 & 0 & 4.312456 & -1.568391 & 0.286367 \\
\hline 35 & 1 & 0 & 4.773962 & -0.624428 & 2.296014 \\
\hline 36 & 1 & 0 & 5.112528 & -2.178588 & -0.117786 \\
\hline & & 1 & 2 & & 3 \\
\hline & & A & A & & A \\
\hline Frequencies & -- & 26.7182 & 38.51 & & 48.7255 \\
\hline Red. masses & -- & 5.5605 & 5.00 & & 5.2341 \\
\hline Frc consts & -- & 0.0023 & 0.00 & & 0.0073 \\
\hline
\end{tabular}




\begin{tabular}{|c|c|c|c|c|c|c|c|c|c|c|}
\hline \multicolumn{2}{|c|}{ IR Inten } & -- & \multicolumn{2}{|c|}{1.4499} & \multicolumn{4}{|c|}{0.4263} & \multicolumn{2}{|c|}{0.5112} \\
\hline Atom & AN & X & $\mathrm{Y}$ & Z & $\mathrm{X}$ & $\mathrm{Y}$ & Z & $\mathrm{x}$ & $\mathrm{Y}$ & Z \\
\hline 1 & 6 & 0.05 & -0.03 & 0.01 & 0.19 & 0.10 & 0.02 & 0.15 & 0.03 & 0.02 \\
\hline 2 & 6 & 0.05 & -0.07 & 0.04 & -0.01 & -0.04 & -0.04 & 0.00 & -0.03 & -0.05 \\
\hline 3 & 6 & 0.05 & -0.04 & 0.02 & 0.05 & 0.05 & 0.03 & 0.05 & 0.02 & 0.01 \\
\hline 4 & 1 & 0.08 & -0.03 & 0.01 & 0.19 & 0.09 & 0.01 & 0.15 & 0.03 & 0.04 \\
\hline 5 & 1 & 0.06 & -0.07 & 0.04 & -0.09 & -0.07 & -0.04 & -0.06 & -0.03 & -0.05 \\
\hline 6 & 1 & 0.06 & -0.02 & 0.00 & 0.00 & 0.07 & 0.11 & 0.01 & 0.06 & 0.06 \\
\hline 7 & 1 & 0.03 & -0.01 & 0.00 & 0.23 & 0.15 & 0.11 & 0.19 & 0.05 & 0.08 \\
\hline 8 & 1 & 0.05 & -0.09 & 0.05 & 0.04 & -0.07 & -0.12 & 0.04 & -0.07 & -0.09 \\
\hline 9 & 8 & 0.03 & -0.06 & 0.03 & 0.26 & 0.08 & -0.09 & 0.21 & 0.01 & -0.07 \\
\hline 10 & 1 & 0.04 & -0.07 & 0.04 & 0.33 & 0.10 & -0.11 & 0.27 & 0.01 & -0.07 \\
\hline 11 & 7 & -0.07 & 0.12 & -0.08 & -0.04 & -0.17 & -0.11 & 0.02 & 0.01 & 0.16 \\
\hline 12 & 1 & -0.08 & 0.14 & -0.09 & -0.12 & -0.21 & -0.10 & 0.11 & -0.03 & 0.19 \\
\hline 13 & 6 & -0.03 & 0.06 & -0.05 & -0.04 & -0.07 & -0.04 & -0.02 & 0.01 & 0.09 \\
\hline 14 & 1 & -0.02 & 0.05 & -0.05 & 0.04 & -0.05 & -0.05 & -0.11 & 0.06 & 0.07 \\
\hline 15 & 1 & 0.03 & 0.06 & -0.07 & -0.12 & -0.08 & -0.02 & 0.01 & 0.02 & 0.16 \\
\hline 16 & 6 & -0.06 & 0.01 & -0.01 & -0.06 & 0.02 & 0.02 & 0.02 & -0.08 & -0.02 \\
\hline 17 & 6 & -0.09 & -0.02 & 0.01 & -0.01 & 0.03 & 0.08 & -0.01 & -0.07 & -0.16 \\
\hline 18 & 1 & -0.07 & 0.00 & -0.01 & -0.11 & 0.05 & 0.01 & 0.08 & -0.17 & 0.02 \\
\hline 19 & 1 & -0.12 & -0.05 & 0.03 & -0.02 & 0.08 & 0.12 & 0.02 & -0.15 & -0.22 \\
\hline 20 & 1 & -0.09 & -0.02 & 0.00 & 0.04 & 0.00 & 0.10 & -0.06 & 0.00 & -0.20 \\
\hline 21 & 6 & -0.15 & 0.13 & -0.07 & 0.06 & -0.18 & -0.15 & -0.02 & 0.01 & 0.10 \\
\hline 22 & 1 & -0.14 & 0.12 & -0.07 & 0.14 & -0.16 & -0.17 & -0.12 & 0.06 & 0.08 \\
\hline 23 & 1 & -0.19 & 0.09 & -0.04 & 0.07 & -0.14 & -0.13 & 0.01 & -0.05 & 0.00 \\
\hline 24 & 1 & -0.18 & 0.18 & -0.10 & 0.06 & -0.27 & -0.20 & 0.01 & 0.01 & 0.16 \\
\hline 25 & 15 & -0.02 & -0.07 & 0.05 & 0.01 & 0.03 & 0.01 & -0.12 & -0.06 & 0.03 \\
\hline 26 & 46 & -0.01 & -0.06 & 0.03 & -0.04 & 0.03 & 0.04 & -0.03 & -0.02 & -0.04 \\
\hline 27 & 6 & 0.05 & 0.07 & -0.03 & 0.00 & 0.02 & -0.02 & -0.08 & 0.00 & 0.06 \\
\hline 28 & 1 & 0.00 & -0.03 & 0.02 & 0.05 & 0.05 & -0.01 & -0.23 & -0.15 & 0.09 \\
\hline 29 & 15 & -0.01 & -0.05 & 0.04 & -0.07 & -0.04 & 0.03 & 0.09 & 0.10 & -0.05 \\
\hline 30 & 1 & 0.01 & -0.04 & 0.02 & -0.06 & 0.03 & 0.06 & -0.02 & 0.00 & -0.05 \\
\hline 31 & 6 & 0.07 & 0.10 & -0.05 & -0.04 & -0.01 & -0.01 & 0.02 & 0.08 & 0.02 \\
\hline 32 & 6 & 0.12 & 0.23 & -0.12 & 0.01 & 0.03 & -0.05 & -0.09 & 0.03 & 0.12 \\
\hline 33 & 1 & 0.05 & 0.08 & -0.04 & -0.08 & -0.05 & 0.03 & 0.20 & 0.18 & -0.06 \\
\hline 34 & 6 & 0.14 & 0.26 & -0.14 & -0.02 & 0.01 & -0.04 & 0.01 & 0.11 & 0.08 \\
\hline 35 & 1 & 0.15 & 0.31 & -0.16 & 0.04 & 0.05 & -0.07 & -0.15 & -0.01 & 0.17 \\
\hline 36 & 1 & 0.19 & 0.37 & -0.20 & -0.03 & 0.00 & -0.05 & 0.06 & 0.17 & 0.08 \\
\hline
\end{tabular}

thermodynamics:

Sum of electronic and zero-point Energies=

$-1369.716962$

Sum of electronic and thermal Enthalpies=

$-1369.694395$

Sum of electronic and thermal Free Energies=

$-1369.768670$

Total free energy in solution:

with all non electrostatic terms

$($ a.u. $)=-1370.053522$

Optimized geometry, three lower frequencies, thermochemistry and PCM energy for 9a.

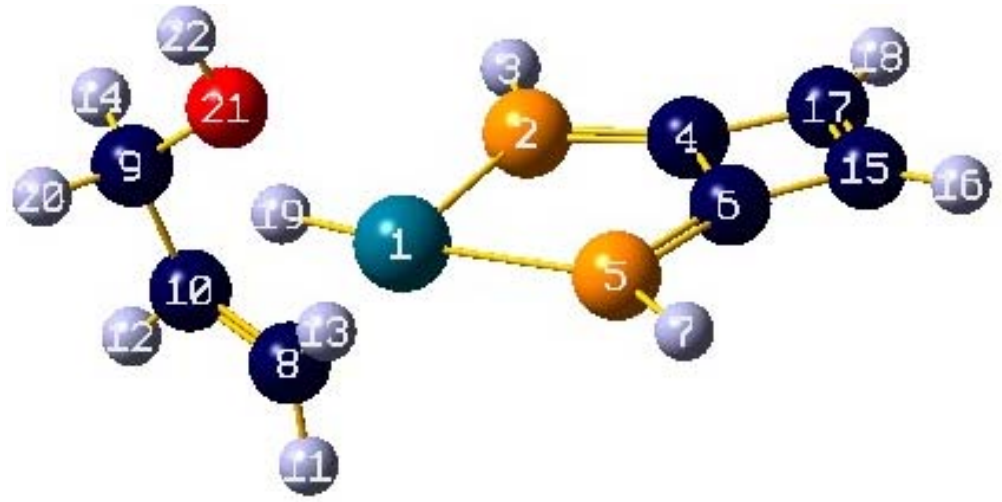




\begin{tabular}{|c|c|c|c|c|c|c|c|c|c|c|}
\hline \multirow{2}{*}{\multicolumn{2}{|c|}{$\begin{array}{l}\text { Center } \\
\text { Number }\end{array}$}} & \multirow{2}{*}{$\begin{array}{l}\text { Atomic } \\
\text { Number }\end{array}$} & \multirow{2}{*}{\multicolumn{2}{|c|}{$\begin{array}{c}\text { Atomic } \\
\text { Type }\end{array}$}} & \multicolumn{6}{|c|}{ Coordinates (Angstroms) } \\
\hline & & & & & & X & $\mathrm{Y}$ & & Z & \\
\hline 1 & & 46 & & 0 & 0. & 99674 & -0.2692 & -0 & 379092 & \\
\hline 2 & & 15 & & 0 & -1.2 & 45016 & -1.5931 & & 035287 & \\
\hline 3 & & 1 & & 0 & -1 & 85520 & -2.9564 & & 121738 & \\
\hline 4 & & 6 & & 0 & -2 & 00268 & -0.6344 & & 231344 & \\
\hline 5 & & 15 & & 0 & -0. & 91592 & 1.5788 & -0 & 137479 & \\
\hline 6 & & 6 & & 0 & -2 & 76788 & 0.8632 & & 153537 & \\
\hline 7 & & 1 & & 0 & -1.1 & 84715 & 2.9781 & -0 & 167494 & \\
\hline 8 & & 6 & & 0 & 2.2 & 98403 & 1.0450 & -0 & 922677 & \\
\hline 9 & & 6 & & 0 & 3. & 45830 & -0.3138 & & 315123 & \\
\hline 10 & & 6 & & 0 & 2. & 37527 & -0.121 & -0 & 545052 & \\
\hline 11 & & 1 & & 0 & 2.7 & 27054 & 1.2570 & -1 & 976299 & \\
\hline 12 & & 1 & & 0 & 3.2 & 66254 & -0.8209 & -1 & 312263 & \\
\hline 13 & & 1 & & 0 & 2.2 & 51504 & 1.8747 & -0 & 221895 & \\
\hline 14 & & 1 & & 0 & 3. & 89581 & -1.3694 & & 113524 & \\
\hline 15 & & 6 & & 0 & -3. & 12949 & $0.927 \varepsilon$ & & 403091 & \\
\hline 16 & & 1 & & 0 & -4 & 13888 & 1.7501 & & 493726 & \\
\hline 17 & & 6 & & 0 & -4 & 25085 & -0.445 & & 472409 & \\
\hline 18 & & 1 & & 0 & -4.8 & 47258 & -1.1326 & & 638740 & \\
\hline 19 & & 1 & & 0 & 1.3 & 83127 & -1.6009 & -0 & 534001 & \\
\hline 20 & & 1 & & 0 & 4.6 & 14495 & -0.0553 & & 717166 & \\
\hline 21 & & 8 & & 0 & $2 . \varepsilon$ & 88483 & 0.5299 & & 733762 & \\
\hline 22 & & 1 & & 0 & 3.3 & 55593 & 0.5157 & & 581403 & \\
\hline & & & 1 & & & 2 & & & 3 & \\
\hline & & & A & & & A & & & A & \\
\hline Frequ & uencies & $s--$ & 21.313 & & & 36.80 & & & 71.66 & \\
\hline Red. & masses & $s--$ & 5.486 & & & 6.59 & & & 7.66 & \\
\hline Frc & consts & -- & 0.001 & & & 0.00 & & & 0.02 & \\
\hline IR Ir & nten & -- & 2.179 & & & 2.20 & & & 0.38 & \\
\hline Atom & AN & X & Y & $\mathrm{z}$ & X & Y & Z & $\mathrm{x}$ & $\mathrm{Y}$ & Z \\
\hline 1 & 46 & -0.01 & 0.06 & 0.07 & -0.01 & -0.04 & 0.06 & 0.03 & 0.02 & -0.03 \\
\hline 2 & 15 & 0.03 & 0.01 & 0.11 & -0.03 & -0.01 & 0.01 & 0.09 & 0.01 & 0.21 \\
\hline 3 & 1 & 0.06 & 0.01 & 0.18 & -0.09 & -0.01 & -0.21 & 0.08 & 0.01 & 0.11 \\
\hline 4 & 6 & -0.01 & -0.02 & -0.01 & -0.04 & 0.01 & -0.11 & 0.06 & 0.00 & 0.06 \\
\hline 5 & 15 & -0.07 & 0.01 & -0.08 & 0.05 & 0.00 & 0.25 & 0.05 & 0.02 & 0.11 \\
\hline 6 & 6 & -0.06 & -0.02 & -0.10 & 0.00 & 0.01 & 0.01 & 0.04 & 0.00 & 0.01 \\
\hline 7 & 1 & -0.11 & 0.00 & -0.20 & 0.07 & 0.00 & 0.26 & 0.01 & 0.01 & -0.05 \\
\hline 8 & 6 & -0.03 & 0.06 & 0.08 & -0.05 & -0.04 & -0.13 & 0.00 & 0.02 & -0.13 \\
\hline 9 & 6 & 0.12 & -0.12 & -0.17 & 0.05 & 0.08 & -0.11 & -0.15 & -0.01 & -0.04 \\
\hline 10 & 6 & -0.01 & 0.01 & -0.10 & -0.01 & 0.00 & -0.09 & -0.02 & 0.01 & -0.10 \\
\hline 11 & 1 & -0.09 & 0.17 & 0.11 & -0.13 & -0.12 & -0.13 & 0.03 & 0.01 & -0.14 \\
\hline 12 & 1 & -0.08 & 0.08 & -0.20 & -0.06 & -0.06 & -0.06 & 0.02 & 0.02 & -0.08 \\
\hline 13 & 1 & 0.05 & -0.01 & 0.17 & -0.02 & 0.01 & -0.18 & -0.03 & 0.03 & -0.14 \\
\hline 14 & 1 & 0.19 & -0.15 & -0.28 & 0.04 & 0.11 & -0.02 & -0.02 & -0.04 & -0.15 \\
\hline 15 & 6 & -0.07 & -0.05 & -0.17 & -0.03 & 0.03 & -0.18 & 0.01 & -0.02 & -0.14 \\
\hline 16 & 1 & -0.11 & -0.07 & -0.26 & -0.03 & 0.03 & -0.23 & -0.02 & -0.03 & -0.27 \\
\hline 17 & 6 & -0.03 & -0.05 & -0.08 & -0.07 & 0.02 & -0.29 & 0.03 & -0.02 & -0.08 \\
\hline 18 & 1 & -0.02 & -0.07 & -0.07 & -0.11 & 0.03 & -0.47 & 0.03 & -0.03 & -0.14 \\
\hline 19 & 1 & 0.07 & 0.09 & 0.15 & -0.08 & -0.06 & -0.08 & -0.01 & 0.02 & -0.20 \\
\hline 20 & 1 & 0.11 & -0.08 & -0.25 & 0.06 & 0.04 & -0.18 & -0.18 & 0.18 & 0.13 \\
\hline 21 & 8 & 0.18 & -0.24 & -0.02 & 0.13 & 0.18 & -0.14 & -0.42 & -0.21 & -0.05 \\
\hline 22 & 1 & 0.26 & -0.31 & -0.07 & 0.16 & 0.22 & -0.16 & -0.52 & -0.18 & 0.00 \\
\hline
\end{tabular}

thermodynamics:

Sum of electronic and zero-point Energies=

$-1157.355719$

Sum of electronic and thermal Enthalpies=

$-1157.340518$

Sum of electronic and thermal Free Energies=

$-1157.398585$

Total free energy in solution:

with all non electrostatic terms (a.u.) = -1157.578918 
Optimized geometry, three lower frequencies, thermochemistry and PCM energy for $\mathbf{T S}_{\mathbf{9 a} \mathbf{a} \mathbf{1 0 a}}$.

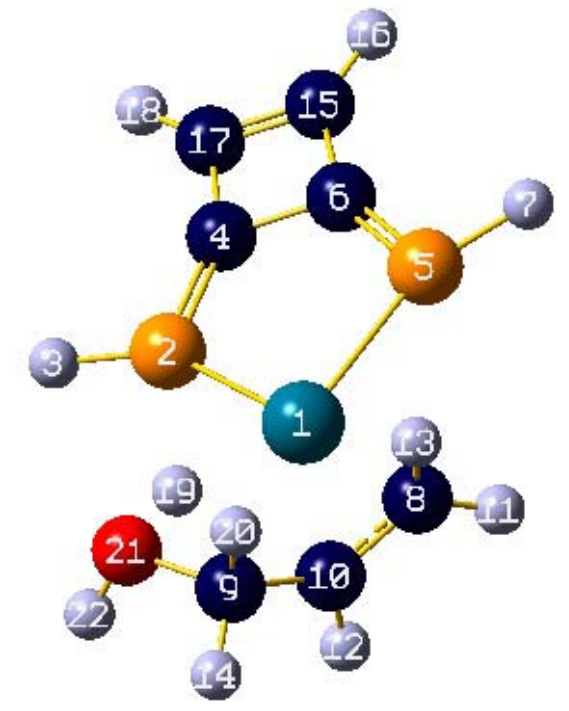

\begin{tabular}{|c|c|c|c|c|c|}
\hline \multirow{2}{*}{$\begin{array}{l}\text { Center } \\
\text { Number }\end{array}$} & \multirow{2}{*}{$\begin{array}{l}\text { Atomic } \\
\text { Number }\end{array}$} & \multirow{2}{*}{$\begin{array}{l}\text { Atomic } \\
\text { Type }\end{array}$} & \multicolumn{3}{|c|}{ Coordinates (Angstroms) } \\
\hline & & & $\mathrm{X}$ & Y & Z \\
\hline 1 & 46 & 0 & -0.646050 & -0.193713 & -0.108570 \\
\hline 2 & 15 & 0 & 0.895703 & 1.547026 & -0.214301 \\
\hline 3 & 1 & 0 & 1.019496 & 2.953027 & -0.276578 \\
\hline 4 & 6 & 0 & 2.438542 & 0.921467 & -0.027286 \\
\hline 5 & 15 & 0 & 1.375802 & -1.640580 & 0.096921 \\
\hline 6 & 6 & 0 & 2.658786 & -0.561231 & 0.114847 \\
\hline 7 & 1 & 0 & 1.967253 & -2.914905 & 0.285968 \\
\hline 8 & 6 & 0 & -2.251656 & -1.694950 & 0.037735 \\
\hline 9 & 6 & 0 & -3.596900 & 0.425661 & 0.397148 \\
\hline 10 & 6 & 0 & -2.825624 & -0.534768 & -0.464083 \\
\hline 11 & 1 & 0 & -1.985547 & -2.507159 & -0.633756 \\
\hline 12 & 1 & 0 & -2.967713 & -0.440914 & -1.541200 \\
\hline 13 & 1 & 0 & -2.339284 & -1.952131 & 1.092813 \\
\hline 14 & 1 & 0 & -4.588884 & 0.655226 & 0.001602 \\
\hline 15 & 6 & 0 & 4.084745 & -0.269577 & 0.221412 \\
\hline 16 & 1 & 0 & 4.961405 & -0.892896 & 0.359741 \\
\hline 17 & 6 & 0 & 3.880919 & 1.088953 & 0.087180 \\
\hline 18 & 1 & 0 & 4.531959 & 1.955996 & 0.075923 \\
\hline 19 & 1 & 0 & -1.717392 & 1.218826 & 0.172058 \\
\hline 20 & 1 & 0 & -3.680850 & 0.088434 & 1.430894 \\
\hline 21 & 8 & 0 & -2.845268 & 1.697498 & 0.476273 \\
\hline 22 & 1 & 0 & -3.125459 & 2.317288 & -0.224442 \\
\hline
\end{tabular}

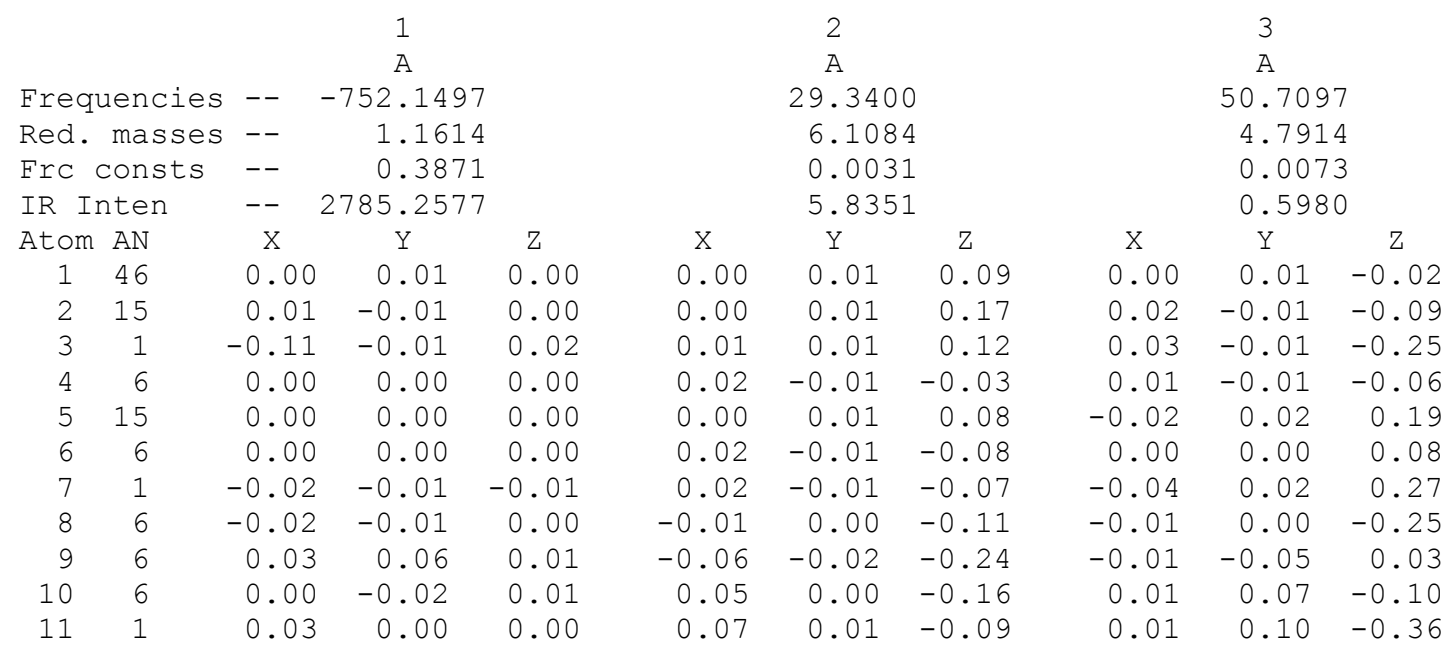




$\begin{array}{rrrrrrrrrrr}12 & 1 & 0.06 & 0.01 & 0.00 & 0.17 & 0.02 & -0.18 & 0.02 & 0.23 & -0.09 \\ 13 & 1 & -0.01 & 0.00 & 0.00 & -0.13 & -0.02 & -0.12 & -0.04 & -0.16 & -0.29 \\ 14 & 1 & -0.01 & -0.10 & -0.01 & -0.02 & -0.01 & -0.36 & 0.01 & 0.02 & 0.04 \\ 15 & 6 & 0.00 & 0.00 & 0.00 & 0.04 & -0.03 & -0.29 & 0.00 & 0.00 & 0.02 \\ 16 & 1 & 0.00 & 0.00 & 0.00 & 0.05 & -0.05 & -0.44 & 0.00 & 0.00 & 0.07 \\ 17 & 6 & 0.00 & 0.00 & 0.00 & 0.04 & -0.03 & -0.23 & 0.02 & -0.02 & -0.12 \\ 18 & 1 & 0.00 & 0.00 & 0.00 & 0.05 & -0.04 & -0.32 & 0.03 & -0.03 & -0.24 \\ 19 & 1 & 0.70 & -0.60 & -0.30 & -0.04 & 0.00 & 0.00 & 0.02 & -0.06 & 0.13 \\ 20 & 1 & 0.00 & -0.01 & -0.01 & -0.19 & -0.04 & -0.25 & -0.05 & -0.23 & -0.03 \\ 21 & 8 & -0.07 & -0.01 & 0.01 & -0.08 & -0.01 & -0.12 & 0.00 & -0.08 & 0.27 \\ 22 & 1 & -0.08 & -0.05 & 0.00 & 0.00 & 0.00 & -0.14 & -0.01 & 0.06 & 0.41\end{array}$

thermodynamics:

Sum of electronic and zero-point Energies=

$-1157.347689$

Sum of electronic and thermal Enthalpies=

$-1157.333218$

Sum of electronic and thermal Free Energies=

$-1157.389258$

Total free energy in solution:

with all non electrostatic terms (a.u.) $=-1157.570075$

Optimized geometry, three lower frequencies, thermochemistry and PCM energy for 10a.

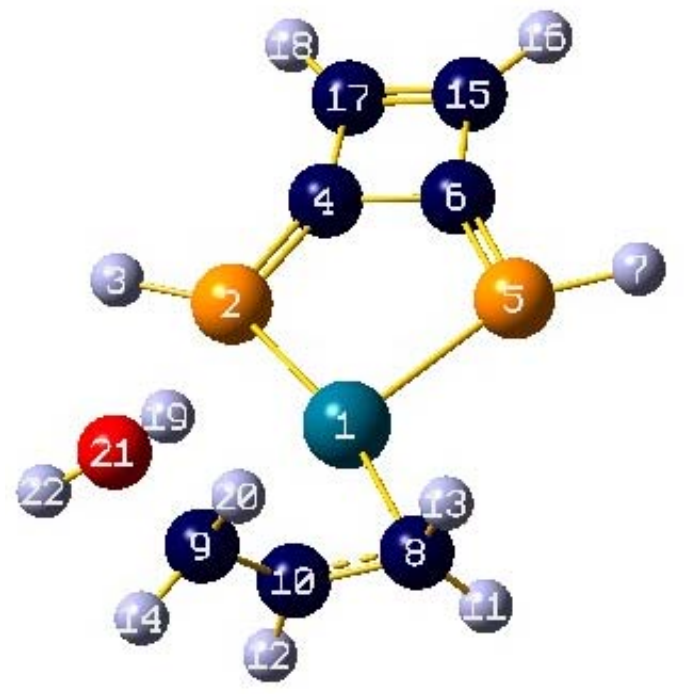

\begin{tabular}{cccrrr}
$\begin{array}{c}\text { Center } \\
\text { Number }\end{array}$ & $\begin{array}{c}\text { Atomic } \\
\text { Number }\end{array}$ & $\begin{array}{c}\text { Atomic } \\
\text { Type }\end{array}$ & \multicolumn{3}{c}{ Coordinates (Angstroms) } \\
-1 & 46 & 0 & -0.642565 & -0.284168 & -0.193822 \\
1 & 15 & 0 & 0.875574 & 1.522678 & -0.365471 \\
2 & 1 & 0 & 1.055126 & 2.907097 & -0.607298 \\
3 & 6 & 0 & 2.414209 & 0.921823 & -0.067183 \\
4 & 15 & 0 & 1.338654 & -1.605146 & 0.245652 \\
5 & 6 & 0 & 2.631297 & -0.538402 & 0.228093 \\
6 & 1 & 0 & 1.896197 & -2.868094 & 0.565264 \\
7 & 6 & 0 & -2.316874 & -1.620250 & -0.120978 \\
8 & 6 & 0 & -3.516800 & 0.492063 & 0.528472 \\
9 & 6 & 0 & -2.823245 & -0.357933 & -0.472322 \\
10 & 1 & 0 & -2.184216 & -2.379324 & -0.888623 \\
11 & 1 & 0 & -3.043125 & -0.149619 & -1.518956 \\
12 & 1 & 0 & -2.421476 & -1.996439 & 0.896296 \\
13 & 1 & 0 & -4.488422 & 0.883926 & 0.221584 \\
14 & 6 & 0 & 4.053044 & -0.232448 & 0.347924 \\
15 & 1 & 0 & 4.925994 & -0.835616 & 0.572623 \\
16 & 6 & 0 & 3.853215 & 1.104274 & 0.065799 \\
17 & 1 & 0 & 4.505537 & 1.965800 & -0.023786 \\
18 & 1 & 0 & -1.726452 & 1.451909 & 0.486193
\end{tabular}




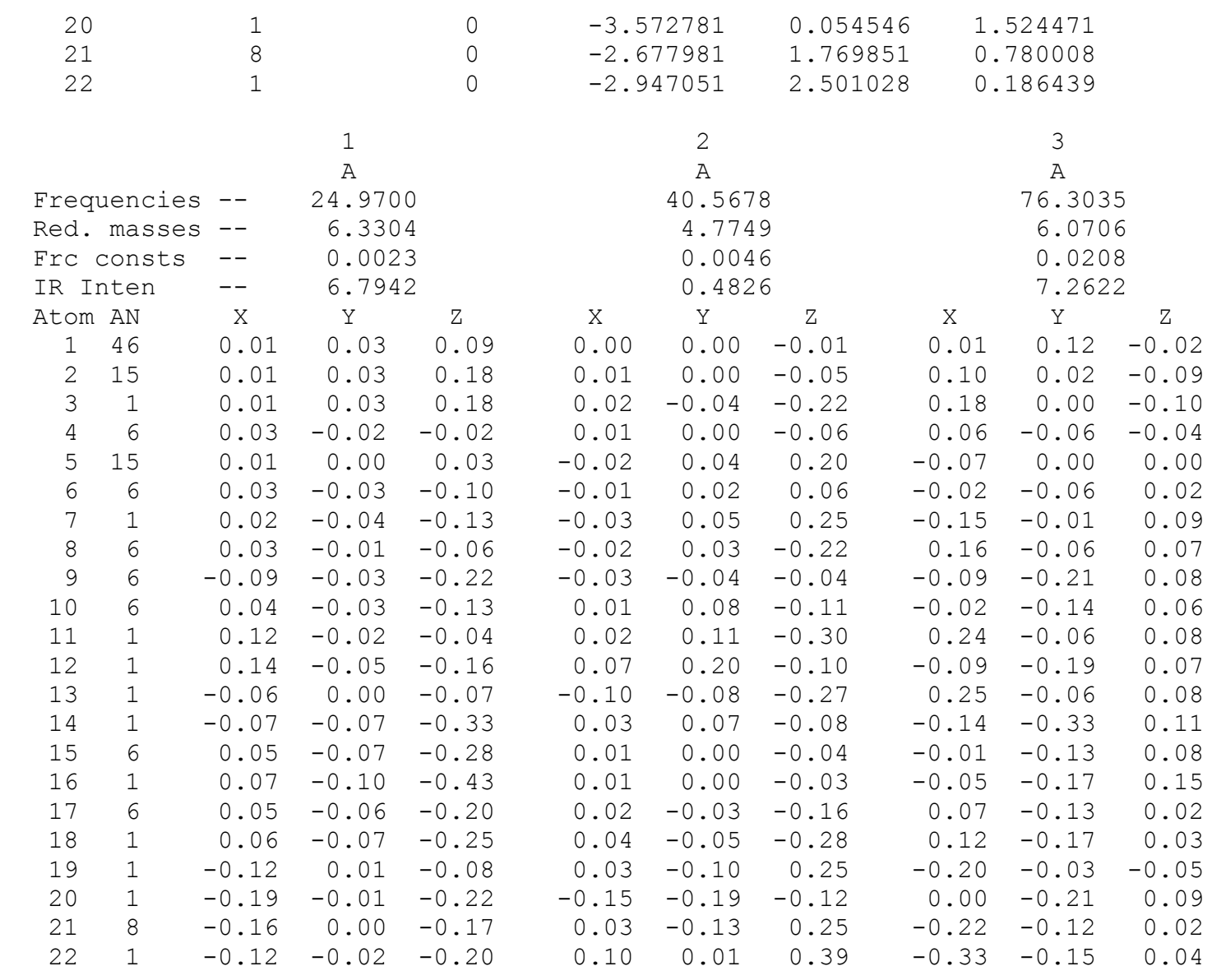

thermodynamics:

Sum of electronic and zero-point Energies=

$-1157.346016$

Sum of electronic and thermal Enthalpies=

$-1157.331130$

Sum of electronic and thermal Free Energies=

$-1157.388314$

Total free energy in solution:

with all non electrostatic terms

$(\mathrm{a} \cdot \mathrm{u} \cdot)=-1157.575980$

Optimized geometry, three lower frequencies, thermochemistry and PCM energy for $\mathbf{T S}_{\mathbf{1 0} \mathbf{a}-\mathbf{1} \text {. }}$.

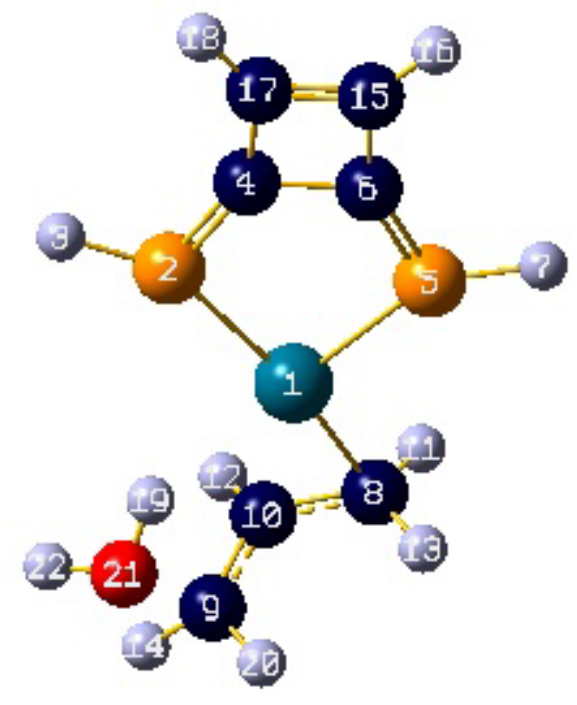




\begin{tabular}{|c|c|c|c|c|c|c|c|c|c|c|}
\hline \multirow{2}{*}{\multicolumn{2}{|c|}{$\begin{array}{l}\text { Center } \\
\text { Number }\end{array}$}} & \multirow{2}{*}{$\begin{array}{l}\text { Atomic } \\
\text { Number }\end{array}$} & \multirow{2}{*}{\multicolumn{2}{|c|}{$\begin{array}{c}\text { Atomic } \\
\text { Type }\end{array}$}} & \multicolumn{6}{|c|}{ Coordinates (Angstroms) } \\
\hline & & & & & & $\mathrm{X}$ & $\mathrm{Y}$ & & Z & \\
\hline 1 & & 46 & & 0 & -.6 & 0179 & -.288470 & & 08834 & \\
\hline 2 & & 15 & & 0 & & 21469 & 1.542914 & & 33463 & \\
\hline 3 & & 1 & & 0 & 1.1 & 50625 & 2.897311 & & 74525 & \\
\hline 4 & & 6 & & 0 & 2. & 36074 & .897962 & & 04841 & \\
\hline 5 & & 15 & & 0 & 1.2 & 56612 & -1.558403 & & 54696 & \\
\hline 6 & & 6 & & 0 & 2. & 1056 & -.550029 & & 73680 & \\
\hline 7 & & 1 & & 0 & 1. & 2926 & -2.840199 & & 18943 & \\
\hline 8 & & 6 & & 0 & -2.2 & 3296 & -1.620731 & & 69907 & \\
\hline 9 & & 6 & & 0 & -3 & 95909 & .384478 & & 76394 & \\
\hline 10 & & 6 & & 0 & -2. & 3431 & -.385739 & & 57860 & \\
\hline 11 & & 1 & & 0 & -2.1 & 3526 & -2.391631 & & 25977 & \\
\hline 12 & & 1 & & 0 & -2 & 92915 & -.171481 & -1 & 14743 & \\
\hline 13 & & 1 & & 0 & -2 & 5780 & -1.996753 & & 42080 & \\
\hline 14 & & 1 & & 0 & -4. & 6314 & .999049 & & 13311 & \\
\hline 15 & & 6 & & 0 & 4 & 34077 & -.294690 & & 65134 & \\
\hline 16 & & 1 & & 0 & $4 . \varepsilon$ & 35189 & -.918487 & & 15555 & \\
\hline 17 & & 6 & & 0 & 3. & 32849 & 1.030227 & & 06927 & \\
\hline 18 & & 1 & & 0 & 4 & 67738 & 1.857856 & & 40648 & \\
\hline 19 & & 1 & & 0 & -1. & 2466 & 1.633716 & & 86939 & \\
\hline 20 & & 1 & & 0 & -3 & 36788 & -.008541 & & 79585 & \\
\hline 21 & & 8 & & 0 & -2 & 2483 & 1.872999 & & 26657 & \\
\hline 22 & & 1 & & 0 & -2. & 50330 & 2.688258 & & 36921 & \\
\hline & & & 1 & & & 2 & & & 3 & \\
\hline & & & A & & & A & & & A & \\
\hline Frequ & dencies & $5--$ & -271.640 & & & 27.35 & & & 43.56 & \\
\hline Red. & masses & $s--$ & 5.837 & & & 6.45 & & & 5.16 & \\
\hline Frc & consts & -- & .253 & & & .00 & & & .00 & \\
\hline IR Ir & nten & -- & 190.0412 & & & 5.18 & & & .19 & \\
\hline Atom & AN & X & $\mathrm{Y}$ & $\mathrm{z}$ & $\mathrm{x}$ & $\mathrm{Y}$ & $\mathrm{Z}$ & $\mathrm{x}$ & $\mathrm{Y}$ & Z \\
\hline 1 & 46 & .00 & .01 & .00 & .01 & .02 & .09 & .00 & .02 & -.01 \\
\hline 2 & 15 & -.02 & -.02 & .01 & .00 & .04 & .18 & .02 & -.01 & -.08 \\
\hline 3 & 1 & -.02 & -.02 & .00 & .00 & .03 & .16 & .03 & -.05 & -.23 \\
\hline 4 & 6 & .00 & .00 & .00 & .02 & -.02 & -.02 & .01 & -.01 & -.07 \\
\hline 5 & 15 & .03 & -.02 & .01 & .01 & .00 & .05 & -.03 & .06 & .21 \\
\hline 6 & 6 & .02 & .00 & .01 & .03 & -.04 & -.10 & -.01 & .02 & .06 \\
\hline 7 & 1 & .10 & .01 & .02 & .02 & -.04 & -.12 & -.04 & .07 & .26 \\
\hline 8 & 6 & -.12 & .07 & .00 & .03 & -.01 & -.08 & -.01 & .02 & -.18 \\
\hline 9 & 6 & .13 & .27 & .19 & -.06 & -.04 & -.21 & -.02 & -.02 & -.07 \\
\hline 10 & 6 & .12 & .17 & .07 & .04 & 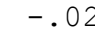 & -.13 & .03 & .07 & -.10 \\
\hline 11 & 1 & -.14 & .10 & -.03 & .11 & -.01 & -.06 & .04 & .09 & -.24 \\
\hline 12 & 1 & -.01 & .16 & .09 & .14 & -.03 & -.15 & .09 & .18 & -.09 \\
\hline 13 & 1 & -.10 & .04 & .00 & -.05 & -.01 & -.09 & -.08 & -.07 & -.23 \\
\hline 14 & 1 & -.11 & -.04 & .15 & -.06 & -.07 & -.29 & .05 & .08 & -.06 \\
\hline 15 & 6 & .01 & .01 & .00 & .05 & -.09 & -.29 & .00 & -.01 & -.02 \\
\hline 16 & 1 & .02 & .02 & .00 & .07 & -.12 & -.44 & -.01 & -.01 & .01 \\
\hline 17 & 6 & .00 & .01 & .00 & .04 & -.07 & -.21 & .02 & -.05 & -.15 \\
\hline 18 & 1 & -.01 & .02 & -.01 & .05 & -.08 & -.28 & .04 & -.08 & -.27 \\
\hline 19 & 1 & -.09 & -.43 & -.21 & -.15 & .05 & -.07 & .02 & -.19 & .29 \\
\hline 20 & 1 & -.01 & .02 & .07 & -.13 & -.03 & -.21 & -.14 & -.15 & -.14 \\
\hline 21 & 8 & -.13 & -.35 & -.20 & -.18 & .01 & -.16 & .01 & -.19 & .26 \\
\hline 22 & 1 & -.13 & -.39 & -.30 & -.17 & -.01 & -.19 & .09 & -.09 & .39 \\
\hline
\end{tabular}

thermodynamics:

Sum of electronic and zero-point Energies=

$-1157.342777$

Sum of electronic and thermal Enthalpies=

$-1157.327705$

Sum of electronic and thermal Free Energies=

$-1157.385289$

Total free energy in solution:

with all non electrostatic terms

$($ a.u. $)=-1157.567828$

Optimized geometry, three lower frequencies, thermochemistry and PCM energy for 11a. 


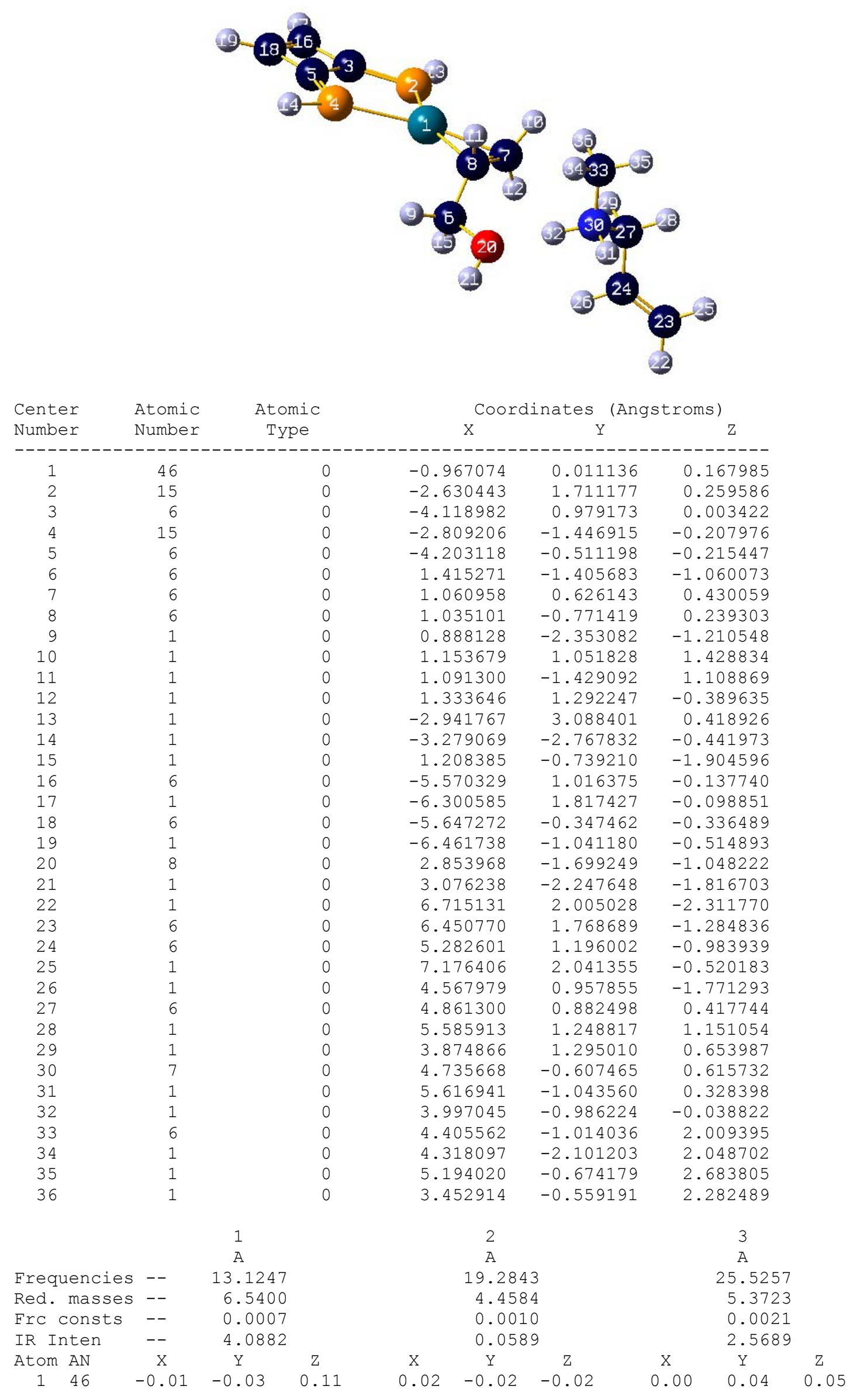




\begin{tabular}{|c|c|c|c|c|c|c|c|c|c|c|}
\hline 2 & 15 & 0.01 & -0.01 & 0.10 & 0.06 & 0.03 & -0.08 & -0.04 & 0.01 & -0.08 \\
\hline 3 & 6 & 0.02 & 0.03 & -0.10 & 0.04 & 0.05 & -0.03 & -0.02 & -0.03 & -0.09 \\
\hline 4 & 15 & -0.01 & 0.01 & -0.02 & -0.01 & 0.01 & 0.05 & 0.02 & -0.04 & 0.20 \\
\hline 5 & 6 & 0.02 & 0.04 & -0.15 & 0.01 & 0.04 & 0.03 & 0.01 & -0.05 & 0.04 \\
\hline 6 & 6 & -0.03 & 0.00 & 0.05 & 0.01 & -0.10 & 0.04 & -0.06 & 0.04 & -0.04 \\
\hline 7 & 6 & 0.00 & -0.05 & 0.12 & 0.03 & -0.04 & -0.04 & 0.00 & 0.09 & -0.10 \\
\hline 8 & 6 & -0.01 & -0.04 & 0.07 & 0.01 & -0.04 & 0.01 & 0.02 & 0.08 & -0.04 \\
\hline 9 & 1 & -0.03 & 0.01 & 0.02 & 0.00 & -0.10 & 0.07 & -0.05 & 0.03 & 0.02 \\
\hline 10 & 1 & 0.01 & -0.08 & 0.13 & 0.03 & 0.00 & -0.05 & 0.05 & 0.13 & -0.12 \\
\hline 11 & 1 & 0.00 & -0.07 & 0.05 & 0.00 & -0.01 & 0.04 & 0.07 & 0.12 & -0.02 \\
\hline 12 & 1 & -0.01 & -0.02 & 0.14 & 0.04 & -0.07 & -0.06 & -0.05 & 0.06 & -0.14 \\
\hline 13 & 1 & 0.03 & -0.01 & 0.08 & 0.09 & 0.04 & -0.12 & -0.06 & 0.02 & -0.25 \\
\hline 14 & 1 & -0.01 & 0.03 & -0.14 & -0.05 & 0.01 & 0.11 & 0.05 & -0.06 & 0.28 \\
\hline 15 & 1 & -0.04 & 0.03 & 0.07 & 0.01 & -0.13 & 0.01 & -0.12 & 0.01 & -0.05 \\
\hline 16 & 6 & 0.04 & 0.07 & -0.28 & 0.04 & 0.07 & 0.00 & -0.01 & -0.06 & -0.19 \\
\hline 17 & 1 & 0.06 & 0.09 & -0.35 & 0.06 & 0.09 & -0.02 & -0.02 & -0.06 & -0.32 \\
\hline 18 & 6 & 0.03 & 0.08 & -0.33 & 0.01 & 0.07 & 0.05 & 0.02 & -0.08 & -0.06 \\
\hline 19 & 1 & 0.04 & 0.10 & -0.46 & -0.01 & 0.08 & 0.09 & 0.04 & -0.10 & -0.05 \\
\hline 20 & 8 & -0.03 & 0.00 & 0.01 & 0.00 & -0.11 & 0.05 & -0.05 & 0.07 & -0.13 \\
\hline 21 & 1 & -0.04 & 0.01 & 0.00 & 0.00 & -0.13 & 0.06 & -0.08 & 0.08 & -0.15 \\
\hline 22 & 1 & -0.14 & 0.03 & -0.18 & -0.33 & 0.31 & 0.03 & 0.11 & 0.09 & 0.15 \\
\hline 23 & 6 & -0.08 & 0.03 & -0.17 & -0.26 & 0.23 & 0.03 & 0.08 & 0.03 & 0.13 \\
\hline 24 & 6 & -0.06 & 0.02 & -0.11 & -0.19 & 0.10 & 0.06 & 0.06 & 0.02 & 0.06 \\
\hline 25 & 1 & -0.05 & 0.04 & -0.21 & -0.25 & 0.27 & 0.02 & 0.06 & -0.04 & 0.17 \\
\hline 26 & 1 & -0.10 & 0.01 & -0.07 & -0.20 & 0.06 & 0.08 & 0.08 & 0.08 & 0.02 \\
\hline 27 & 6 & 0.01 & 0.03 & -0.08 & -0.10 & -0.01 & 0.06 & 0.02 & -0.07 & 0.02 \\
\hline 28 & 1 & 0.04 & 0.03 & -0.12 & -0.09 & 0.00 & 0.05 & 0.01 & -0.13 & 0.07 \\
\hline 29 & 1 & 0.02 & 0.02 & -0.03 & -0.12 & -0.10 & 0.13 & 0.02 & -0.08 & 0.02 \\
\hline 30 & 7 & 0.03 & 0.03 & -0.07 & 0.02 & -0.03 & -0.02 & 0.00 & -0.08 & -0.08 \\
\hline 31 & 1 & 0.02 & 0.03 & -0.11 & 0.04 & 0.05 & -0.08 & -0.01 & -0.08 & -0.12 \\
\hline 32 & 1 & 0.00 & 0.02 & -0.04 & 0.01 & -0.05 & 0.00 & -0.02 & -0.02 & -0.10 \\
\hline 33 & 6 & 0.09 & 0.03 & -0.06 & 0.11 & -0.13 & -0.02 & 0.01 & -0.18 & -0.11 \\
\hline 34 & 1 & 0.11 & 0.03 & -0.05 & 0.19 & -0.14 & -0.08 & -0.03 & -0.18 & -0.19 \\
\hline 35 & 1 & 0.11 & 0.05 & -0.09 & 0.12 & -0.11 & -0.04 & 0.04 & -0.26 & -0.10 \\
\hline 36 & 1 & 0.09 & 0.01 & -0.02 & 0.09 & -0.21 & 0.05 & 0.03 & -0.16 & -0.0 \\
\hline
\end{tabular}

thermodynamics:

Sum of electronic and zero-point Energies=

Sum of electronic and thermal Enthalpies=

$-1369.738602$

Sum of electronic and thermal Free Energies=

$-1369.817394$

Total free energy in solution:

with all non electrostatic terms (a.u.) = -1370.108038

Optimized geometry, three lower frequencies, thermochemistry and PCM energy for $\mathbf{T S}_{11 \mathrm{a} 1 \mathrm{a}}$.

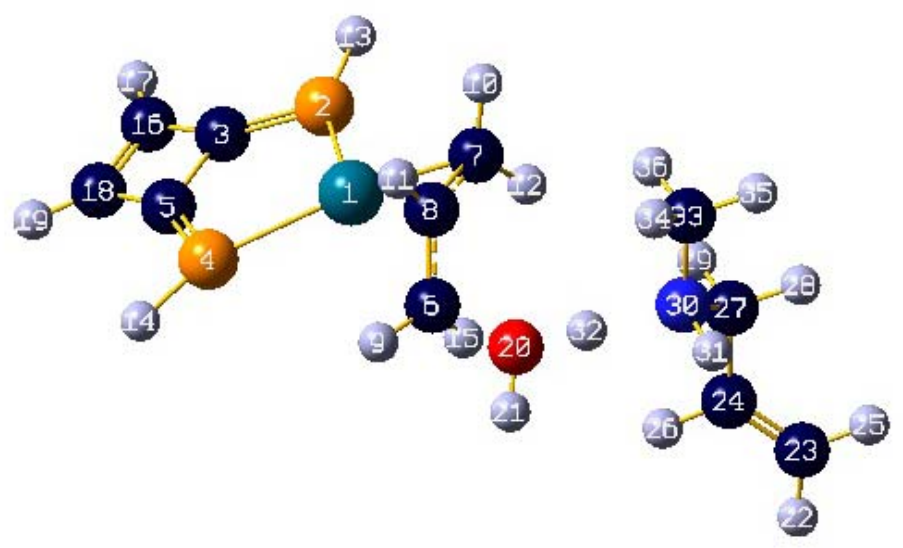




\begin{tabular}{|c|c|c|c|c|c|c|c|c|c|c|}
\hline \multirow{2}{*}{\multicolumn{2}{|c|}{$\begin{array}{l}\text { Center } \\
\text { Number }\end{array}$}} & \multirow{2}{*}{$\begin{array}{l}\text { Atomic } \\
\text { Number }\end{array}$} & \multirow{2}{*}{\multicolumn{2}{|c|}{$\begin{array}{c}\text { Atomic } \\
\text { Type }\end{array}$}} & \multicolumn{6}{|c|}{ Coordinates (Angstroms) } \\
\hline & & & & & & $\mathrm{x}$ & $\mathrm{Y}$ & & Z & \\
\hline 1 & & 46 & & 0 & 1.0 & 39426 & -0.228 & -0 & 323823 & \\
\hline 2 & & 15 & & 0 & 2.6 & 67619 & 1.373 & -0 & 943993 & \\
\hline 3 & & 6 & & 0 & 4.0 & 92343 & $0.989^{\circ}$ & -0 & 148109 & \\
\hline 4 & & 15 & & 0 & 2.7 & 67620 & -1.101 & & 86592 & \\
\hline 5 & & 6 & & 0 & 4.1 & 36109 & -0.171 & & 312727 & \\
\hline 6 & & 6 & & 0 & -1.2 & 69316 & -1.275 & & 589145 & \\
\hline 7 & & 6 & & 0 & -0.7 & 57854 & 0.150 & -1 & 380878 & \\
\hline 8 & & 6 & & 0 & -0.8 & 92385 & -1.122 & -0 & 774620 & \\
\hline 9 & & 1 & & 0 & -0.9 & 34891 & -2.187 & & 103677 & \\
\hline 10 & & 1 & & 0 & -0.6 & 25253 & 0.218 & -2 & 458034 & \\
\hline 11 & & 1 & & 0 & -0.7 & 57410 & -2.024 & -1 & 367501 & \\
\hline 12 & & 1 & & 0 & -1.1 & 91498 & 1.032 & -0 & 909096 & \\
\hline 13 & & 1 & & 0 & 2.9 & 70847 & 2.480 & -1 & 775096 & \\
\hline 14 & & 1 & & 0 & 3.1 & 69869 & -2.097 & & 012349 & \\
\hline 15 & & 1 & & 0 & $-1 \cdot 3$ & 77835 & -0.391 & & 210588 & \\
\hline 16 & & 6 & & 0 & 5.5 & 3802 & 1.204 & & 147300 & \\
\hline 17 & & 1 & & 0 & 6.2 & 34640 & 1.925 & -0 & 202295 & \\
\hline 18 & & 6 & & 0 & 5.5 & 43726 & 0.141 & & 027403 & \\
\hline 19 & & 1 & & 0 & 6.3 & 18573 & -0.302 & & 642501 & \\
\hline 20 & & 8 & & 0 & -3.0 & 97818 & -1.7892 & & 619589 & \\
\hline 21 & & 1 & & 0 & -3.3 & 55438 & -2.073 & & 512455 & \\
\hline 22 & & 1 & & 0 & -6.0 & 58010 & 2.192 & & 054420 & \\
\hline 23 & & 6 & & 0 & -6.0 & 45008 & 2.017 & & 982054 & \\
\hline 24 & & 6 & & 0 & -5.0 & 54497 & 1.340 & & 395985 & \\
\hline 25 & & 1 & & 0 & -6.8 & 73119 & 2.432 & & 409925 & \\
\hline 26 & & 1 & & 0 & -4.2 & 36770 & 0.950 & & 003586 & \\
\hline 27 & & 6 & & 0 & -4.9 & 74622 & 1.083 & -0 & 079231 & \\
\hline 28 & & 1 & & 0 & -5.7 & 95936 & 1.5942 & -0 & 601894 & \\
\hline 29 & & 1 & & 0 & -4.0 & 34666 & 1.474 & -0 & 491391 & \\
\hline 30 & & 7 & & 0 & -4.9 & 99903 & -0.367 & -0 & 372421 & \\
\hline 31 & & 1 & & 0 & -5.8 & 52232 & -0.752 & & 037810 & \\
\hline 32 & & 1 & & 0 & -3.8 & 73968 & -1.125 & & 237513 & \\
\hline 33 & & 6 & & 0 & -4.9 & 96800 & -0.6603 & -1 & 315291 & \\
\hline 34 & & 1 & & 0 & -5.0 & 67276 & -1.7407 & -1 & 965742 & \\
\hline 35 & & 1 & & 0 & -5.8 & 27948 & -0.1688 & -2 & 337048 & \\
\hline 36 & & 1 & & 0 & -4.0 & 54976 & -0.3110 & -2 & 248529 & \\
\hline & & & 1 & & & 2 & & & 3 & \\
\hline & & & A & & & A & & & A & \\
\hline Freq & uencies & $s--$ & -336.915 & & & 12.208 & & & 15.653 & \\
\hline Red. & masses & $s--$ & 6.263 & & & 4.94 & & & $5.22 \varepsilon$ & \\
\hline Frc & consts & -- & 0.418 & & & 0.000 & & & 0.000 & \\
\hline IR I & hten & -- & 1686.569 & & & 0.465 & & & 0.815 & \\
\hline Atom & AN & X & $\mathrm{Y}$ & Z & X & Y & Z & X & $\mathrm{Y}$ & Z \\
\hline 1 & 46 & -0.01 & 0.00 & 0.02 & -0.01 & -0.04 & -0.03 & -0.03 & 0.03 & 0.05 \\
\hline 2 & 15 & 0.00 & -0.01 & -0.01 & -0.04 & 0.01 & 0.00 & -0.06 & 0.09 & 0.12 \\
\hline 3 & 6 & -0.01 & -0.01 & 0.00 & -0.06 & 0.06 & 0.06 & -0.02 & 0.01 & 0.00 \\
\hline 4 & 15 & 0.01 & 0.00 & 0.01 & -0.03 & 0.01 & 0.01 & 0.03 & -0.07 & -0.09 \\
\hline 5 & 6 & 0.00 & 0.00 & 0.00 & -0.05 & 0.06 & 0.06 & 0.03 & -0.07 & -0.09 \\
\hline 6 & 6 & 0.53 & 0.10 & -0.05 & -0.01 & -0.07 & -0.06 & 0.00 & -0.05 & 0.03 \\
\hline 7 & 6 & 0.04 & 0.04 & -0.05 & -0.01 & -0.07 & -0.06 & -0.05 & 0.09 & 0.12 \\
\hline 8 & 6 & 0.01 & 0.01 & -0.09 & 0.00 & -0.07 & -0.06 & -0.03 & 0.05 & 0.03 \\
\hline 9 & 1 & 0.17 & -0.03 & -0.09 & 0.00 & -0.07 & -0.06 & 0.01 & -0.08 & -0.04 \\
\hline 10 & 1 & 0.00 & 0.07 & -0.05 & 0.00 & -0.07 & -0.06 & -0.08 & 0.17 & 0.12 \\
\hline 11 & 1 & 0.00 & 0.02 & -0.09 & 0.02 & -0.07 & -0.05 & -0.03 & 0.09 & -0.03 \\
\hline 12 & 1 & 0.04 & 0.02 & -0.01 & -0.02 & -0.07 & -0.06 & -0.05 & 0.06 & 0.19 \\
\hline 13 & 1 & -0.06 & -0.02 & -0.03 & -0.06 & 0.02 & 0.02 & -0.09 & 0.15 & 0.18 \\
\hline 14 & 1 & -0.07 & -0.01 & 0.01 & -0.02 & 0.03 & 0.03 & 0.08 & -0.16 & -0.20 \\
\hline 15 & 1 & 0.20 & 0.05 & -0.04 & -0.02 & -0.07 & -0.06 & 0.01 & -0.09 & 0.09 \\
\hline 16 & 6 & -0.01 & 0.01 & 0.00 & -0.08 & 0.12 & 0.12 & 0.00 & -0.03 & -0.06 \\
\hline 17 & 1 & -0.01 & 0.01 & 0.00 & -0.09 & 0.15 & 0.15 & -0.01 & -0.01 & -0.05 \\
\hline 18 & 6 & 0.00 & 0.00 & 0.00 & -0.07 & 0.12 & 0.12 & 0.04 & -0.10 & -0.15 \\
\hline 19 & 1 & 0.00 & 0.01 & -0.01 & -0.08 & 0.15 & 0.15 & 0.08 & -0.16 & -0.24 \\
\hline 20 & 8 & -0.18 & -0.21 & 0.13 & 0.00 & -0.08 & -0.08 & 0.00 & -0.06 & 0.03 \\
\hline 21 & 1 & -0.31 & -0.12 & 0.11 & 0.00 & -0.13 & -0.09 & 0.00 & -0.05 & 0.04 \\
\hline
\end{tabular}




$\begin{array}{rrrrrrrrrrr}22 & 1 & 0.00 & -0.03 & 0.01 & 0.38 & 0.15 & 0.06 & 0.16 & 0.21 & -0.17 \\ 23 & 6 & 0.00 & 0.01 & 0.01 & 0.31 & 0.13 & 0.06 & 0.14 & 0.14 & -0.15 \\ 24 & 6 & -0.01 & 0.01 & -0.02 & 0.22 & 0.04 & 0.01 & 0.09 & 0.04 & -0.12 \\ 25 & 1 & 0.01 & 0.03 & 0.02 & 0.31 & 0.19 & 0.10 & 0.16 & 0.15 & -0.17 \\ 26 & 1 & -0.03 & -0.05 & -0.04 & 0.22 & -0.02 & -0.03 & 0.07 & 0.03 & -0.10 \\ 27 & 6 & -0.04 & 0.03 & -0.03 & 0.11 & 0.01 & 0.01 & 0.06 & -0.06 & -0.10 \\ 28 & 1 & 0.01 & 0.16 & 0.00 & 0.12 & 0.06 & 0.05 & 0.08 & -0.06 & -0.13 \\ 29 & 1 & -0.01 & -0.01 & -0.01 & 0.12 & -0.05 & -0.05 & 0.07 & -0.12 & -0.14 \\ 30 & 7 & -0.15 & 0.07 & -0.05 & 0.00 & 0.01 & 0.03 & 0.01 & -0.08 & -0.01 \\ 31 & 1 & -0.19 & 0.12 & -0.10 & 0.00 & 0.07 & 0.09 & 0.00 & -0.02 & 0.02 \\ 32 & 1 & 0.15 & -0.35 & 0.25 & 0.00 & -0.05 & -0.03 & 0.00 & -0.07 & 0.02 \\ 33 & 6 & -0.03 & 0.04 & -0.01 & -0.10 & -0.01 & 0.04 & -0.01 & -0.16 & 0.00 \\ 34 & 1 & -0.04 & 0.04 & 0.01 & -0.17 & 0.00 & 0.05 & -0.04 & -0.17 & 0.07 \\ 35 & 1 & 0.03 & 0.03 & -0.14 & -0.11 & 0.03 & 0.08 & -0.01 & -0.17 & -0.02 \\ 36 & 1 & 0.01 & 0.00 & 0.04 & -0.11 & -0.06 & -0.03 & -0.01 & -0.21 & -0.03\end{array}$

thermodynamics:

Sum of electronic and zero-point Energies=

$-1369.740089$

Sum of electronic and thermal Enthalpies=

$-1369.717362$

Sum of electronic and thermal Free Energies=

$-1369.796022$

Total free energy in solution:

with all non electrostatic terms

$($ a.u. $)=-1370.073421$

Optimized geometry, three lower frequencies, thermochemistry and PCM energy for 12a.

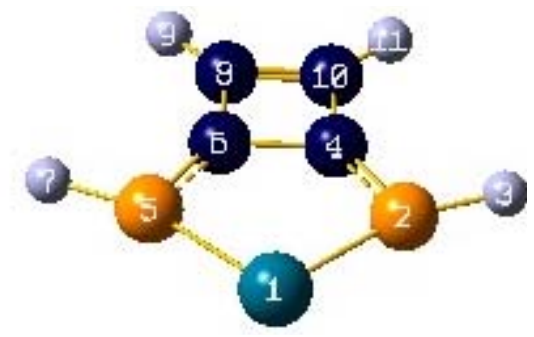

\begin{tabular}{cccrrr} 
Center & Atomic & Atomic & \multicolumn{3}{c}{ Coordinates (Angstroms) } \\
Number & Number & Type & X & Y & Z \\
-1 & 46 & 0 & 1.459085 & 0.000001 & -0.000892 \\
1 & 15 & 0 & -0.124129 & 1.668698 & 0.002006 \\
3 & 1 & 0 & -0.667350 & 2.996513 & -0.000757 \\
4 & 6 & 0 & -1.544062 & 0.757857 & 0.001012 \\
5 & 15 & 0 & -0.124116 & -1.668690 & 0.001779 \\
6 & 6 & 0 & -1.544059 & -0.757867 & 0.001001 \\
7 & 1 & 0 & -0.667307 & -2.996518 & -0.000652 \\
8 & 6 & 0 & -2.998159 & -0.691914 & -0.001228 \\
9 & 1 & 0 & -3.776472 & -1.448058 & -0.002731 \\
10 & 6 & 0 & -2.998160 & 0.691899 & -0.002020 \\
11 & 1 & 0 & -3.776475 & 1.448040 & -0.004202
\end{tabular}

\begin{tabular}{|c|c|c|c|c|c|c|c|c|c|}
\hline & \multicolumn{3}{|c|}{1} & \multicolumn{3}{|c|}{2} & \multicolumn{3}{|c|}{3} \\
\hline & \multicolumn{3}{|c|}{ A } & \multicolumn{3}{|c|}{ A } & \multicolumn{3}{|c|}{ A } \\
\hline Frequencies & -- & \multicolumn{2}{|c|}{49.9512} & \multicolumn{3}{|c|}{169.5745} & \multicolumn{3}{|c|}{187.0644} \\
\hline Red. masses & -- & \multicolumn{2}{|c|}{10.6413} & \multicolumn{3}{|c|}{3.3718} & \multicolumn{3}{|c|}{19.4880} \\
\hline Frc consts & -- & \multicolumn{2}{|c|}{0.0156} & \multicolumn{3}{|c|}{0.0571} & \multicolumn{3}{|c|}{0.4018} \\
\hline IR Inten & -- & \multicolumn{2}{|c|}{5.1537} & \multicolumn{3}{|c|}{0.0003} & \multicolumn{3}{|c|}{0.5366} \\
\hline Atom AN & X & Y & Z & X & $\mathrm{Y}$ & Z & X & Y & Z \\
\hline 146 & 0.00 & 0.00 & 0.13 & 0.00 & 0.00 & 0.00 & 0.28 & 0.00 & 0.00 \\
\hline 15 & 0.00 & 0.00 & -0.31 & 0.00 & 0.00 & 0.10 & -0.21 & -0.20 & 0.00 \\
\hline 1 & 0.00 & 0.00 & 0.04 & 0.00 & 0.00 & -0.40 & -0.09 & -0.15 & 0.00 \\
\hline 6 & 0.00 & 0.00 & -0.09 & 0.00 & 0.00 & -0.16 & -0.32 & -0.02 & 0.00 \\
\hline 15 & 0.00 & 0.00 & -0.31 & 0.00 & 0.00 & -0.10 & -0.21 & 0.20 & 0.00 \\
\hline 6 & 0.00 & 0.00 & -0.09 & 0.00 & 0.00 & 0.16 & -0.32 & 0.02 & \\
\hline
\end{tabular}




$\begin{array}{rrrrrrrrrrr}7 & 1 & 0.00 & 0.00 & 0.04 & 0.00 & 0.00 & 0.40 & -0.09 & 0.15 & 0.00 \\ 8 & 6 & 0.00 & 0.00 & 0.29 & 0.00 & 0.00 & 0.23 & -0.35 & 0.00 & 0.00 \\ 9 & 1 & 0.00 & 0.00 & 0.55 & 0.00 & 0.00 & 0.50 & -0.35 & 0.00 & 0.00 \\ 10 & 6 & 0.00 & 0.00 & 0.29 & 0.00 & 0.00 & -0.23 & -0.35 & 0.00 & 0.00 \\ 11 & 1 & 0.00 & 0.00 & 0.55 & 0.00 & 0.00 & -0.50 & -0.35 & 0.00 & 0.00\end{array}$

thermodynamics:

Sum of electronic and thermal Enthalpies=

$-964.016508$

Sum of electronic and thermal Free Energies=

$-964.007456$

Total free energy in solution:

with all non electrostatic terms (a.u.) $=\quad-964.080568$

Optimized geometry, three lower frequencies, thermochemistry and PCM energy for 13a.

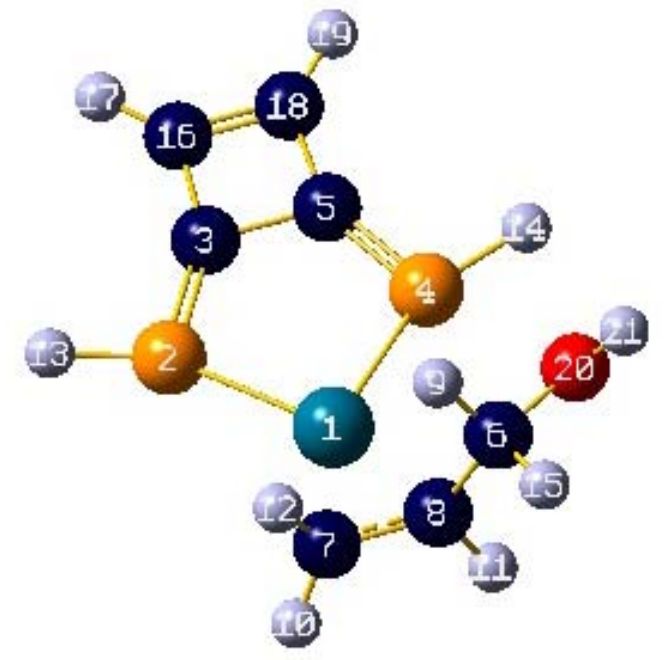

\begin{tabular}{|c|c|c|c|c|c|}
\hline \multirow{2}{*}{$\begin{array}{l}\text { Center } \\
\text { Number }\end{array}$} & \multirow{2}{*}{$\begin{array}{l}\text { Atomic } \\
\text { Number }\end{array}$} & \multirow{2}{*}{$\begin{array}{l}\text { Atomic } \\
\text { Type }\end{array}$} & \multicolumn{3}{|c|}{ Coordinates (Angstroms) } \\
\hline & & & $\mathrm{X}$ & $\mathrm{Y}$ & Z \\
\hline 1 & 46 & 0 & 0.613224 & -0.530832 & $-0.24612 \xi$ \\
\hline 2 & 15 & 0 & -1.449966 & -1.601135 & 0.165703 \\
\hline 3 & 6 & 0 & -2.610979 & -0.385158 & 0.240236 \\
\hline 4 & 15 & 0 & -0.683600 & 1.405712 & -0.605041 \\
\hline 5 & 6 & 0 & -2.249277 & 1.037929 & -0.113431 \\
\hline 6 & 6 & 0 & 3.174158 & 0.868301 & 0.790961 \\
\hline 7 & 6 & 0 & 2.506761 & -1.508512 & 0.186901 \\
\hline 8 & 6 & 0 & 2.762757 & -0.188881 & -0.19400 \\
\hline 9 & 1 & 0 & 2.839872 & 0.591470 & $1.80220^{\circ}$ \\
\hline 10 & 1 & 0 & 2.629097 & -2.322633 & -0.525032 \\
\hline 11 & 1 & 0 & 3.056418 & 0.014286 & -1.22531 \\
\hline 12 & 1 & 0 & 2.527935 & -1.797266 & 1.23762 \\
\hline 13 & 1 & 0 & -2.170356 & -2.736675 & 0.64533 \\
\hline 14 & 1 & 0 & -0.716446 & 2.823291 & -0.74360 \\
\hline 15 & 1 & 0 & 4.276470 & 0.928941 & 0.80541 \\
\hline 16 & 6 & 0 & -3.944359 & 0.103591 & 0.57820 \\
\hline 17 & 1 & 0 & -4.840233 & -0.379516 & 0.95409 \\
\hline 18 & 6 & 0 & -3.612165 & 1.405351 & 0.26149 \\
\hline 19 & 1 & 0 & -4.144892 & 2.350337 & 0.29179 \\
\hline 20 & 8 & 0 & 2.637736 & 2.132747 & 0.40483 \\
\hline 21 & 1 & 0 & 3.074043 & 2.819657 & 0.92865 \\
\hline
\end{tabular}

$\begin{array}{cccc} & 1 & 2 & 3 \\ \text { Frequencies -- } & 19.6652 & \text { A } & \text { A } \\ & & 47.9687 & 54.2879\end{array}$




\begin{tabular}{|c|c|c|c|c|c|c|c|c|c|c|}
\hline \multirow{2}{*}{\multicolumn{2}{|c|}{$\begin{array}{l}\text { Red. masses } \\
\text { Frc consts }\end{array}$}} & $s--$ & \multicolumn{2}{|c|}{5.5966} & \multicolumn{3}{|c|}{5.3785} & \multicolumn{3}{|c|}{5.7253} \\
\hline & & -- & 0.00 & & & $0.00^{\circ}$ & & & 0.009 & \\
\hline IR In & nten & -- & 2.18 & & & 0.78 & & & 3.024 & \\
\hline Atom & AN & X & Y & Z & $\mathrm{X}$ & Y & Z & $\mathrm{X}$ & Y & Z \\
\hline 1 & 46 & 0.00 & 0.04 & 0.07 & 0.00 & 0.04 & -0.04 & 0.02 & -0.06 & 0.02 \\
\hline 2 & 15 & 0.01 & 0.02 & 0.05 & 0.05 & 0.03 & 0.17 & -0.01 & 0.03 & 0.08 \\
\hline 3 & 6 & -0.05 & -0.03 & -0.06 & 0.01 & 0.00 & 0.09 & 0.01 & 0.06 & 0.00 \\
\hline 4 & 15 & -0.02 & 0.03 & 0.15 & -0.07 & -0.03 & -0.15 & 0.09 & -0.01 & 0.05 \\
\hline 5 & 6 & -0.06 & -0.02 & -0.02 & -0.05 & -0.03 & -0.09 & 0.06 & 0.05 & 0.00 \\
\hline 6 & 6 & 0.16 & -0.07 & -0.15 & 0.04 & -0.12 & 0.10 & -0.07 & 0.05 & -0.09 \\
\hline 7 & 6 & 0.02 & -0.03 & -0.16 & -0.01 & -0.02 & -0.17 & 0.12 & 0.02 & -0.18 \\
\hline 8 & 6 & 0.02 & -0.03 & -0.14 & 0.02 & 0.01 & -0.03 & -0.02 & 0.07 & -0.13 \\
\hline 9 & 1 & 0.26 & -0.10 & -0.13 & 0.03 & -0.24 & 0.07 & 0.13 & -0.11 & -0.07 \\
\hline 10 & 1 & -0.08 & -0.01 & -0.20 & -0.05 & 0.05 & -0.26 & 0.13 & 0.07 & -0.24 \\
\hline 11 & 1 & -0.09 & 0.00 & -0.17 & 0.02 & 0.12 & 0.00 & -0.11 & 0.15 & -0.13 \\
\hline 12 & 1 & 0.12 & -0.07 & -0.17 & 0.01 & -0.14 & -0.20 & 0.22 & -0.04 & -0.20 \\
\hline 13 & 1 & -0.01 & -0.03 & -0.06 & 0.11 & 0.06 & 0.33 & -0.08 & 0.05 & 0.03 \\
\hline 14 & 1 & -0.06 & 0.03 & 0.10 & -0.14 & -0.05 & -0.34 & 0.15 & -0.01 & 0.02 \\
\hline 15 & 1 & 0.16 & -0.11 & -0.27 & 0.04 & -0.15 & 0.11 & -0.08 & 0.26 & -0.24 \\
\hline 16 & 6 & -0.11 & -0.08 & -0.23 & 0.01 & -0.01 & 0.08 & 0.00 & 0.09 & -0.09 \\
\hline 17 & 1 & -0.14 & -0.12 & -0.35 & 0.04 & 0.01 & 0.17 & -0.03 & 0.12 & -0.14 \\
\hline 18 & 6 & -0.12 & -0.07 & -0.19 & -0.05 & -0.03 & -0.10 & 0.05 & 0.08 & -0.08 \\
\hline 19 & 1 & -0.17 & -0.10 & -0.28 & -0.09 & -0.05 & -0.21 & 0.07 & 0.09 & -0.13 \\
\hline 20 & 8 & 0.15 & -0.04 & -0.05 & 0.07 & -0.06 & 0.27 & -0.37 & -0.01 & 0.12 \\
\hline 21 & 1 & 0.23 & -0.07 & -0.08 & 0.06 & -0.14 & 0.38 & -0.43 & 0.02 & 0.13 \\
\hline
\end{tabular}

thermodynamics:

Sum of electronic and zero-point Energies=

$-1157.009004$

Sum of electronic and thermal Enthalpies=

$-1156.994110$

Sum of electronic and thermal Free Energies=

$-1157.051795$

Total free energy in solution:

with all non electrostatic terms

Total free energy in solution:

with all non electrostatic terms

$$
\begin{aligned}
& (\mathrm{a} \cdot \mathrm{u} \cdot)=\quad-1157.162936 \\
& (\mathrm{a} \cdot \mathrm{u} .)=\quad-1157.157878
\end{aligned}
$$

Optimized geometry, three lower frequencies, thermochemistry and PCM energy for 14a.

\begin{tabular}{|c|c|c|c|c|c|}
\hline Center & Atomic & Atomic & \multicolumn{3}{|c|}{ Coordinates (Angstroms) } \\
\hline Number & Number & Type & $\mathrm{X}$ & $Y$ & Z \\
\hline 1 & 15 & 0 & -0.994124 & -1.550528 & -0.027070 \\
\hline 2 & 1 & 0 & -1.170355 & -2.947459 & 0.017114 \\
\hline 3 & 6 & 0 & -2.464913 & -0.755252 & -0.009358 \\
\hline
\end{tabular}

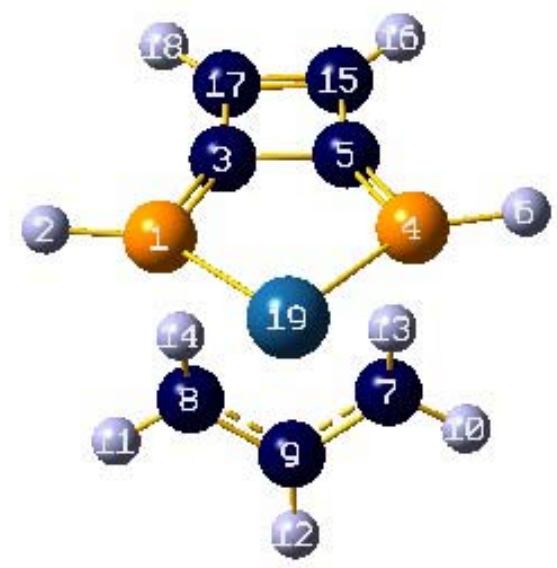




$\begin{array}{rr}4 & 15 \\ 5 & 6 \\ 6 & 1 \\ 7 & 6 \\ 8 & 6 \\ 9 & 6 \\ 10 & 1 \\ 11 & 1 \\ 12 & 1 \\ 13 & 1 \\ 14 & 1 \\ 15 & 6 \\ 16 & 1 \\ 17 & 6 \\ 18 & 1 \\ 19 & 78\end{array}$

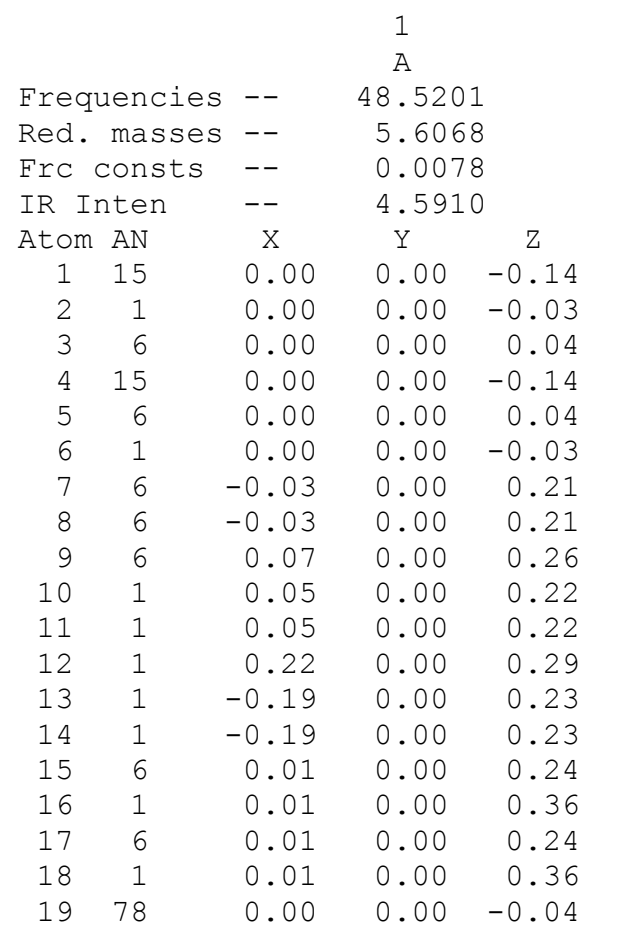

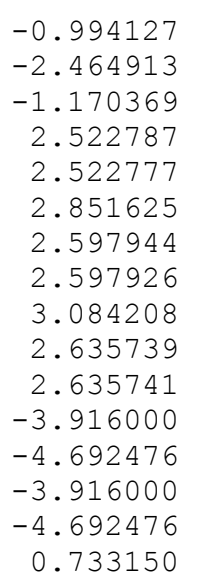

$$
\begin{array}{r}
1.550531 \\
0.755249 \\
2.947459 \\
1.215325 \\
-1.215332 \\
0.000002 \\
2.147160 \\
-2.147155 \\
0.000010 \\
1.300627 \\
-1.300657 \\
0.692160 \\
1.448829 \\
-0.692163 \\
-1.448832 \\
0.000000
\end{array}
$$

\begin{tabular}{rrr}
\multicolumn{3}{c}{2} \\
\multicolumn{3}{c}{97.5071} \\
\multicolumn{3}{c}{3.0638} \\
\multicolumn{3}{c}{0.0172} \\
\multicolumn{3}{c}{1.4821} \\
X & \multicolumn{1}{c}{$\mathrm{Y}$} & \multicolumn{1}{c}{$\mathrm{Z}$} \\
0.01 & 0.00 & \multicolumn{1}{c}{-0.08} \\
0.03 & 0.00 & -0.18 \\
0.01 & -0.02 & -0.06 \\
-0.01 & 0.00 & 0.08 \\
-0.01 & -0.02 & 0.06 \\
-0.03 & 0.00 & 0.18 \\
0.04 & -0.01 & -0.23 \\
-0.04 & -0.01 & 0.23 \\
0.00 & -0.15 & 0.00 \\
0.06 & -0.13 & -0.41 \\
-0.06 & -0.13 & 0.41 \\
0.00 & -0.37 & 0.00 \\
0.08 & 0.20 & -0.25 \\
-0.08 & 0.20 & 0.25 \\
-0.01 & -0.03 & 0.07 \\
-0.01 & -0.04 & 0.15 \\
0.01 & -0.03 & -0.07 \\
0.01 & -0.04 & -0.15 \\
0.00 & 0.02 & 0.00
\end{tabular}

\begin{tabular}{rrr}
\multicolumn{3}{c}{3} \\
\multicolumn{3}{c}{ A } \\
& 114.2319 \\
& 4.4223 \\
& 0.0340 \\
\multicolumn{3}{c}{5.4850} \\
\multicolumn{1}{c}{ X } & \multicolumn{1}{c}{$Z$} \\
-0.06 & \multicolumn{1}{c}{-0.02} & \multicolumn{1}{c}{-0.03} \\
-0.16 & -0.01 & -0.07 \\
-0.02 & 0.05 & -0.03 \\
0.06 & -0.02 & 0.03 \\
0.02 & 0.05 & 0.03 \\
0.16 & -0.01 & 0.07 \\
-0.19 & 0.19 & -0.09 \\
0.19 & 0.19 & 0.09 \\
0.00 & 0.19 & 0.00 \\
-0.34 & 0.16 & -0.18 \\
0.34 & 0.16 & 0.18 \\
0.00 & 0.14 & 0.00 \\
-0.17 & 0.31 & -0.11 \\
0.17 & 0.31 & 0.11 \\
0.02 & 0.11 & 0.03 \\
0.05 & 0.14 & 0.07 \\
-0.02 & 0.11 & -0.03 \\
-0.05 & 0.14 & -0.07 \\
0.00 & -0.06 & 0.00
\end{tabular}

thermodynamics:

Sum of electronic and zero-point Energies=

$-1073.450385$

Sum of electronic and thermal Enthalpies=

$-1073.437917$

Sum of electronic and thermal Free Energies=

Total free energy in solution:

with all non electrostatic terms

$(\mathrm{a} \cdot \mathrm{u})=$.

Optimized geometry, three lower frequencies, thermochemistry and PCM energy for $\mathbf{T S}_{\mathbf{1 4 a - 1 5 a}}$. 


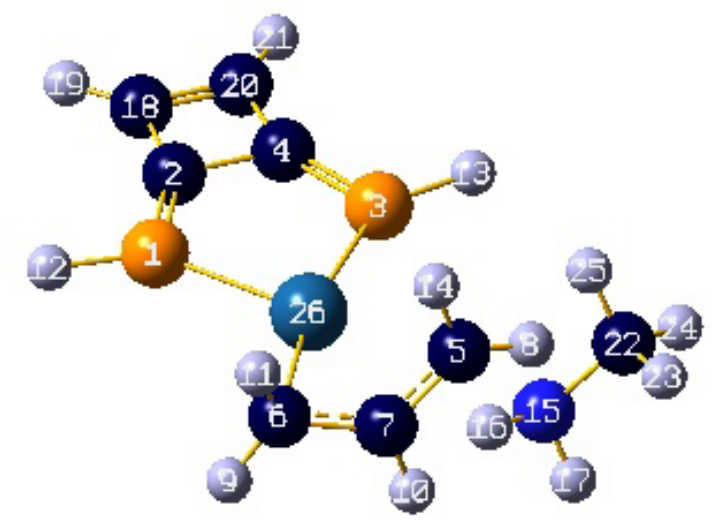

\begin{tabular}{|c|c|c|c|c|c|c|c|c|c|c|}
\hline \multirow{2}{*}{\multicolumn{2}{|c|}{$\begin{array}{l}\text { Center } \\
\text { Number }\end{array}$}} & \multirow{2}{*}{$\begin{array}{l}\text { Atomic } \\
\text { Number }\end{array}$} & \multirow{2}{*}{\multicolumn{2}{|c|}{$\begin{array}{c}\text { Atomic } \\
\text { Type }\end{array}$}} & \multicolumn{6}{|c|}{ Coordinates (Angstroms) } \\
\hline & & & & & & $\mathrm{X}$ & Y & & Z & \\
\hline \multicolumn{2}{|l|}{1} & \multicolumn{2}{|l|}{15} & 0 & \multicolumn{2}{|c|}{-1.908046} & -1.283643 & \multicolumn{3}{|c|}{.466407} \\
\hline 2 & & 6 & & 0 & \multicolumn{2}{|c|}{-3.136362} & -.151342 & \multicolumn{3}{|c|}{.369044} \\
\hline 3 & & 15 & & 0 & \multicolumn{2}{|c|}{-1.170229} & 1.605744 & \multicolumn{3}{|c|}{-.372127} \\
\hline 4 & & 6 & & 0 & \multicolumn{2}{|c|}{-2.772578} & 1.260518 & \multicolumn{3}{|c|}{-.027321} \\
\hline 5 & & 6 & & 0 & \multicolumn{2}{|c|}{2.555715} & .454126 & \multicolumn{3}{|c|}{-.034806} \\
\hline 6 & & 6 & & 0 & \multicolumn{2}{|c|}{1.584538} & -1.839312 & \multicolumn{3}{|c|}{-.072494} \\
\hline 7 & & 6 & & 0 & 2.1 & 36978 & -.694087 & & 4705 & \\
\hline 8 & & 1 & & 0 & 2. & 7411 & 1.404283 & & 48603 & \\
\hline 9 & & 1 & & 0 & 1.3 & 8332 & -2.736967 & & 5816 & \\
\hline 10 & & 1 & & 0 & 2.2 & 38435 & -.698816 & -1 & 3112 & \\
\hline 11 & & 1 & & 0 & $1 . \varepsilon$ & 56835 & -2.031300 & & 66698 & \\
\hline 12 & & 1 & & 0 & -2 & 36730 & -2.541939 & & 2494 & \\
\hline 13 & & 1 & & 0 & -1. & 2897 & 2.974072 & & 1339 & \\
\hline 14 & & 1 & & 0 & 2 & 38832 & .490852 & & 4766 & \\
\hline 15 & & 7 & & 0 & 4.7 & 20510 & .404944 & & 4305 & \\
\hline 16 & & 1 & & 0 & 4. & 8921 & -.476297 & & 08911 & \\
\hline 17 & & 1 & & 0 & 5 & 5567 & .328286 & & 08479 & \\
\hline 18 & & 6 & & 0 & -4.5 & 5707 & .297710 & & 65700 & \\
\hline 19 & & 1 & & 0 & -5 & 3252 & -.201145 & & 23299 & \\
\hline 20 & & 6 & & 0 & 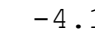 & 33123 & 1.590352 & & 02029 & \\
\hline 21 & & 1 & & 0 & -4. & 8470 & 2.504659 & & 39957 & \\
\hline 22 & & 6 & & 0 & 5.3 & 21193 & 1.542173 & & 48956 & \\
\hline 23 & & 1 & & 0 & 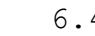 & -3539 & 1.471139 & & 20226 & \\
\hline 24 & & 1 & & 0 & 5. & 57008 & 2.467356 & & 23887 & \\
\hline 25 & & 1 & & 0 & 4. & 9681 & 1.596515 & & 60820 & \\
\hline 26 & & 78 & & 0 & & 0813 & -.345867 & & 34828 & \\
\hline & & & 1 & & & 2 & & & 3 & \\
\hline & & & A & & & A & & & A & \\
\hline Freq & dencies & $s--$ & -245.177 & & & 25.13 & & & 30.12 & \\
\hline Red. & masses & $s--$ & 6.067 & & & 3.86 & & & 3.30 & \\
\hline Frc & consts & -- & .214 & & & .00 & & & .00 & \\
\hline IR I & nten & -- & 719.976 & & & 1.55 & & & 4.52 & \\
\hline Atom & AN & X & Y & $\mathrm{z}$ & X & $\mathrm{Y}$ & Z & X & $\mathrm{Y}$ & Z \\
\hline 1 & 15 & .00 & .01 & .02 & -.02 & -.02 & -.08 & -.03 & .00 & -.06 \\
\hline 2 & 6 & -.01 & .00 & .01 & -.02 & -.03 & -.11 & .01 & .04 & .03 \\
\hline 3 & 15 & .00 & .00 & -.01 & .01 & .03 & .12 & .03 & .00 & -.01 \\
\hline 4 & 6 & .00 & .00 & .00 & -.01 & .00 & -.01 & .04 & .04 & .05 \\
\hline 5 & 6 & .52 & .05 & .10 & .02 & -.03 & .05 & -.01 & -.02 & .08 \\
\hline 6 & 6 & .06 & .10 & .01 & -.02 & -.01 & -.05 & .00 & -.03 & .03 \\
\hline 7 & 6 & .03 & .17 & .07 & -.01 & .02 & .01 & .03 & -.03 & .06 \\
\hline 8 & 1 & .21 & .11 & .15 & .02 & .00 & .10 & .00 & -.02 & .08 \\
\hline 9 & 1 & .10 & .13 & -.05 & -.03 & .02 & -.09 & .02 & -.03 & .02 \\
\hline 10 & 1 & .03 & .20 & .07 & -.03 & .07 & .00 & .07 & -.03 & .07 \\
\hline 11 & 1 & .06 & .03 & -.01 & -.01 & -.07 & -.06 & -.05 & -.03 & .05 \\
\hline 12 & 1 & -.03 & .02 & .02 & -.04 & -.05 & -.21 & -.05 & .01 & -.05 \\
\hline 13 & 1 & -.06 & .00 & -.04 & .02 & .04 & .19 & .07 & .01 & .04 \\
\hline 14 & 1 & .18 & .08 & .09 & .04 & -.08 & .06 & -.07 & -.02 & .07 \\
\hline
\end{tabular}




$\begin{array}{rrrrrrrrrrr}15 & 7 & -.27 & -.05 & -.03 & .02 & -.07 & -.02 & -.03 & .02 & .17 \\ 16 & 1 & -.032 & -.07 & -.05 & .02 & .02 & .14 & -.03 & .14 & .40 \\ 17 & 1 & -.34 & -.07 & -.06 & -.03 & -.26 & -.02 & .00 & -.21 & .20 \\ 18 & 6 & -.01 & .01 & -.01 & -.04 & -.05 & -.20 & .03 & .09 & .11 \\ 19 & 1 & -.01 & .01 & -.02 & -.05 & -.07 & -.30 & .02 & .11 & .14 \\ 20 & 6 & .00 & .01 & .00 & -.02 & -.02 & -.10 & .06 & .09 & .13 \\ 21 & 1 & .00 & .01 & .00 & -.02 & -.02 & -.10 & .08 & .11 & .19 \\ 22 & 6 & -.14 & -.07 & -.05 & .09 & .06 & -.27 & -.07 & .21 & -.10 \\ 23 & 1 & -.15 & -.02 & -.15 & .10 & .03 & -.31 & -.07 & .24 & -.04 \\ 24 & 1 & -.09 & -.06 & -.05 & .10 & -.04 & -.05 & -.06 & .07 & -.034 \\ 25 & 1 & -.07 & -.06 & -.03 & .15 & .27 & -.26 & -.01 & .46 & -.12 \\ 26 & 78 & -.01 & -.02 & -.01 & .00 & .01 & .04 & .00 & -.03 & -.03\end{array}$

thermodynamics:

Sum of electronic and zero-point Energies=

$-1169.204096$

Sum of electronic and thermal Enthalpies=

$-1169.187552$

Sum of electronic and thermal Free Energies=

$-1169.249676$

Total free energy in solution:

with all non electrostatic terms

$(\mathrm{a} \cdot \mathrm{u})=$.

Optimized geometry, three lower frequencies, thermochemistry and PCM energy for 15a.

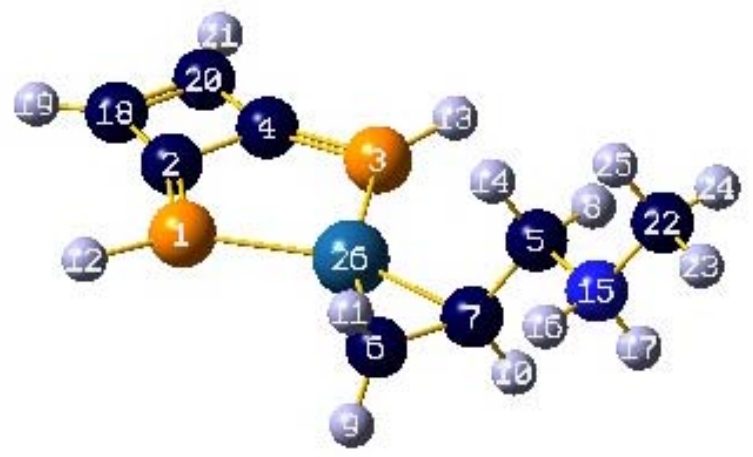

\begin{tabular}{|c|c|c|c|c|c|}
\hline \multirow{2}{*}{$\begin{array}{l}\text { Center } \\
\text { Number }\end{array}$} & \multirow{2}{*}{$\begin{array}{l}\text { Atomic } \\
\text { Number }\end{array}$} & \multirow{2}{*}{$\begin{array}{l}\text { Atomic } \\
\text { Type }\end{array}$} & \multicolumn{3}{|c|}{ Coordinates (Angstroms) } \\
\hline & & & $\mathrm{X}$ & Y & Z \\
\hline 1 & 15 & 0 & -1.865476 & -1.354941 & 0.446554 \\
\hline 2 & 6 & 0 & -3.147673 & -0.277263 & 0.430570 \\
\hline 3 & 15 & 0 & -1.286491 & 1.565686 & -0.363188 \\
\hline 4 & 6 & 0 & -2.862747 & 1.152501 & 0.031781 \\
\hline 5 & 6 & 0 & 2.955593 & 0.682265 & 0.229703 \\
\hline 6 & 6 & 0 & 1.783541 & -1.533285 & -0.258190 \\
\hline 7 & 6 & 0 & 2.136829 & -0.187123 & -0.668655 \\
\hline 8 & 1 & 0 & 2.900616 & 1.745319 & -0.021397 \\
\hline 9 & 1 & 0 & 1.725509 & -2.318416 & -1.011359 \\
\hline 10 & 1 & 0 & 2.286704 & 0.002781 & -1.735079 \\
\hline 11 & 1 & 0 & 2.081187 & -1.896232 & 0.729575 \\
\hline 12 & 1 & 0 & -2.355378 & -2.622267 & 0.838384 \\
\hline 13 & 1 & 0 & -1.287388 & 2.939696 & -0.706629 \\
\hline 14 & 1 & 0 & 2.712017 & 0.547704 & 1.286860 \\
\hline 15 & 7 & 0 & 4.446866 & 0.307605 & 0.108345 \\
\hline 16 & 1 & 0 & 4.515744 & -0.698343 & 0.297058 \\
\hline 17 & 1 & 0 & 4.718976 & 0.415528 & -0.874129 \\
\hline 18 & 6 & 0 & -4.540393 & 0.112708 & 0.590087 \\
\hline 19 & 1 & 0 & -5.434358 & -0.426969 & 0.882392 \\
\hline 20 & 6 & 0 & -4.279933 & 1.420584 & 0.223772 \\
\hline 21 & 1 & 0 & -4.889632 & 2.310673 & 0.114897 \\
\hline 22 & 6 & 0 & 5.382028 & 1.069782 & 0.985544 \\
\hline
\end{tabular}




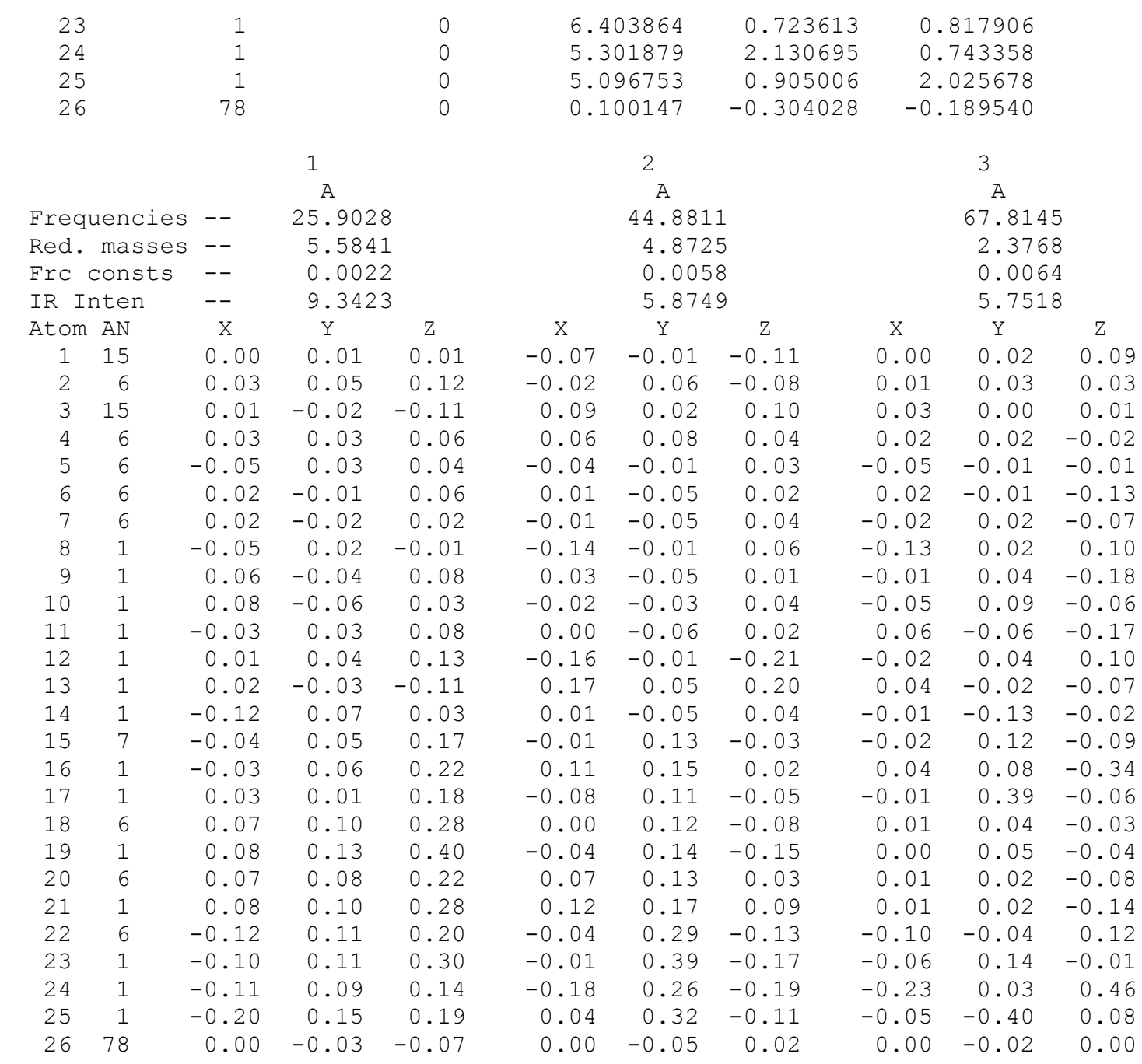

thermodynamics:

Sum of electronic and zero-point Energies=

$-1169.218723$

Sum of electronic and thermal Enthalpies=

$-1169.202762$

Sum of electronic and thermal Free Energies=

$-1169.262936$

Total free energy in solution:

with all non electrostatic terms

$(\mathrm{a} \cdot \mathrm{u} \cdot)=-1169.496537$

Optimized geometry, three lower frequencies, thermochemistry and PCM energy for $\mathbf{T S}_{\mathbf{1 5}}$ a-16a. 


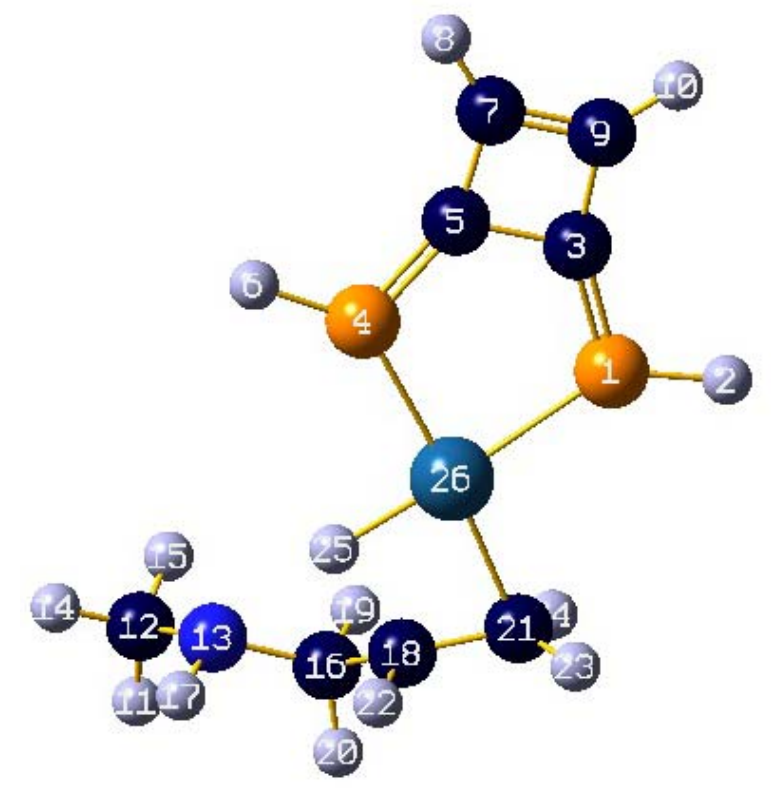

\begin{tabular}{|c|c|c|c|c|c|}
\hline \multirow{2}{*}{$\begin{array}{l}\text { Center } \\
\text { Number }\end{array}$} & \multirow{2}{*}{$\begin{array}{l}\text { Atomic } \\
\text { Number }\end{array}$} & \multirow{2}{*}{$\begin{array}{l}\text { Atomic } \\
\text { Type }\end{array}$} & \multicolumn{3}{|c|}{ Coordinates (Angstroms) } \\
\hline & & & $\mathrm{x}$ & $\mathrm{Y}$ & Z \\
\hline 1 & 15 & 0 & -1.831186 & -1.311129 & .436210 \\
\hline 2 & 1 & 0 & -2.316511 & -2.565186 & .855534 \\
\hline 3 & 6 & 0 & -3.053671 & -.172652 & .381015 \\
\hline 4 & 15 & 0 & -1.119850 & 1.576230 & -.432683 \\
\hline 5 & 6 & 0 & -2.708122 & 1.234630 & -.041019 \\
\hline 6 & 1 & 0 & -.964913 & 2.928759 & -.792651 \\
\hline 7 & 6 & 0 & -4.112638 & 1.564891 & .146646 \\
\hline 8 & 1 & 0 & -4.682339 & 2.479665 & .027159 \\
\hline 9 & 6 & 0 & -4.428709 & .275302 & .532490 \\
\hline 10 & 1 & 0 & -5.343103 & -.222314 & .835023 \\
\hline 11 & 1 & 0 & 5.452617 & 1.469123 & 1.116492 \\
\hline 12 & 6 & 0 & 4.470797 & 1.912987 & .885139 \\
\hline 13 & 7 & 0 & 3.672485 & 1.122485 & -.049469 \\
\hline 14 & 1 & 0 & 4.631849 & 2.908212 & .461805 \\
\hline 15 & 1 & 0 & 3.917479 & 2.033131 & 1.822624 \\
\hline 16 & 6 & 0 & 3.423026 & -.236706 & .390828 \\
\hline 17 & 1 & 0 & 4.127947 & 1.116261 & -.959963 \\
\hline 18 & 6 & 0 & 2.370959 & -.899618 & -.473141 \\
\hline 19 & 1 & 0 & 3.080412 & -.209865 & 1.431073 \\
\hline 20 & 1 & 0 & 4.315867 & -.889819 & .361080 \\
\hline 21 & 6 & 0 & 1.529291 & -1.952917 & .014392 \\
\hline 22 & 1 & 0 & 2.603153 & -.928425 & -1.540254 \\
\hline 23 & 1 & 0 & 1.258627 & -2.756328 & -.669140 \\
\hline 24 & 1 & 0 & 1.630391 & -2.250325 & 1.058702 \\
\hline 25 & 1 & 0 & 1.589504 & .452182 & -.597689 \\
\hline 26 & 78 & 0 & .183482 & -.330186 & -.181212 \\
\hline
\end{tabular}

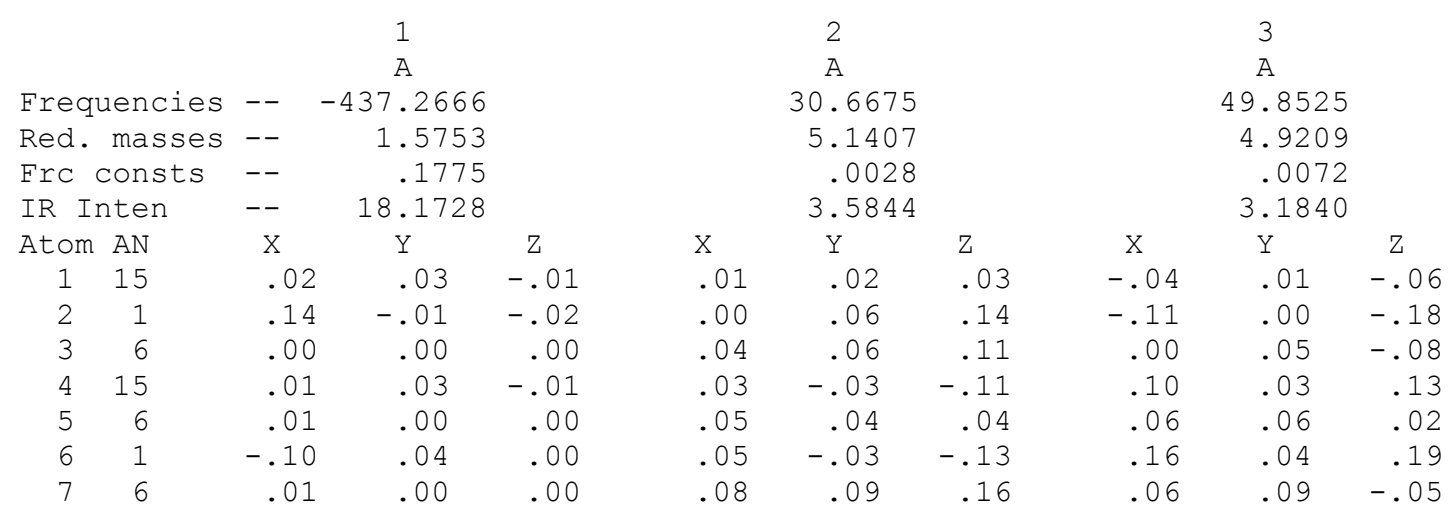




\begin{tabular}{|c|c|c|c|c|c|c|c|c|c|}
\hline 8 & .00 & .00 & .00 & .10 & .11 & .19 & .10 & .12 & -.03 \\
\hline 9 & .00 & .00 & .00 & .07 & .11 & .22 & .00 & .08 & -.15 \\
\hline 10 & .00 & .00 & -.01 & .08 & .15 & .33 & -.03 & .08 & -.24 \\
\hline 11 & .02 & .02 & .03 & -.22 & .18 & .27 & -.21 & .30 & -.06 \\
\hline 12 & .02 & .01 & .01 & -.23 & .11 & .16 & -.26 & .16 & -.09 \\
\hline 13 & .04 & .01 & .01 & -.09 & .04 & .10 & -.13 & .05 & -.10 \\
\hline 14 & .01 & .01 & .01 & -.25 & .11 & .15 & -.39 & .18 & -.09 \\
\hline 15 & .00 & .01 & .01 & -.33 & .10 & .10 & -.30 & .09 & -.10 \\
\hline 16 & .05 & .00 & .01 & -.05 & .04 & .10 & .01 & .02 & -.11 \\
\hline 17 & .07 & .00 & .02 & .00 & .05 & .14 & -.11 & .10 & -.09 \\
\hline 18 & -.01 & .14 & -.05 & .02 & -.02 & .05 & -.01 & -.02 & -.06 \\
\hline 19 & .01 & .01 & -.01 & -.11 & .04 & .08 & .08 & -.03 & -.09 \\
\hline 20 & .04 & -.01 & .03 & -.02 & .08 & .16 & .06 & .09 & -.18 \\
\hline 21 & -.05 & .07 & -.01 & .01 & -.02 & .04 & .02 & -.04 & -.04 \\
\hline 22 & .18 & -.11 & .00 & .09 & -.05 & .07 & -.05 & -.01 & -.07 \\
\hline 23 & .08 & .01 & .00 & .05 & -.04 & .05 & .01 & -.04 & -.03 \\
\hline 24 & .04 & .05 & -.02 & -.03 & .01 & .05 & .06 & -.04 & -.04 \\
\hline 25 & .54 & -.74 & -.16 & .01 & -.04 & -.06 & .02 & -.04 & .02 \\
\hline 78 & -.01 & -.02 & .00 & .01 & -.03 & -.06 & .01 & -.04 & .04 \\
\hline
\end{tabular}

thermodynamics:

Sum of electronic and zero-point Energies=

Sum of electronic and thermal Enthalpies=

$-1169.181245$

Sum of electronic and thermal Free Energies=

$-1169.240444$

Total free energy in solution:

with all non electrostatic terms

$(\mathrm{a} \cdot \mathrm{u})=$.

Optimized geometry, three lower frequencies, thermochemistry and PCM energy for 16a.

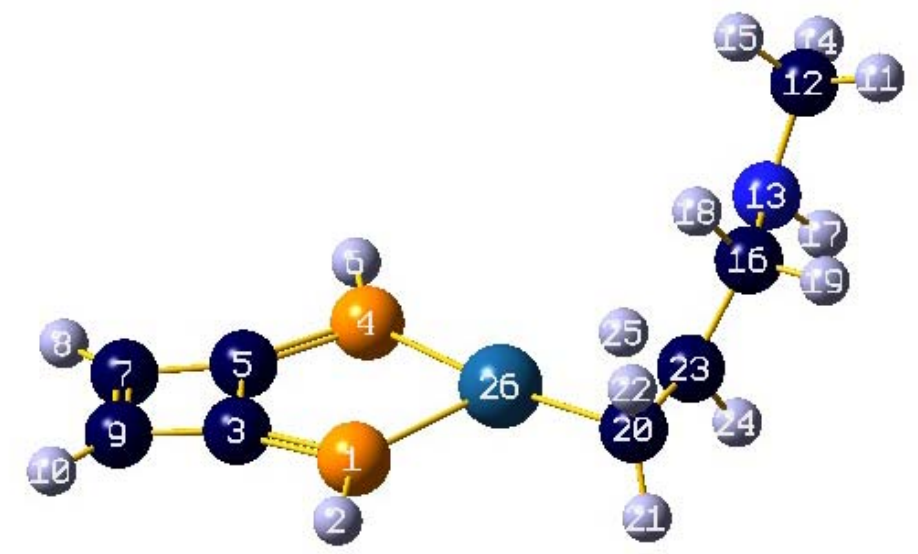

\begin{tabular}{|c|c|c|c|c|c|}
\hline \multirow{2}{*}{$\begin{array}{l}\text { Center } \\
\text { Number }\end{array}$} & \multirow{2}{*}{$\begin{array}{l}\text { Atomic } \\
\text { Number }\end{array}$} & \multirow{2}{*}{$\begin{array}{c}\text { Atomic } \\
\text { Type }\end{array}$} & \multicolumn{3}{|c|}{ Coordinates (Angstroms) } \\
\hline & & & $\mathrm{X}$ & $\mathrm{Y}$ & Z \\
\hline 1 & 15 & 0 & -1.814011 & -1.280687 & .452157 \\
\hline 2 & 1 & 0 & -2.220419 & -2.557374 & .880435 \\
\hline 3 & 6 & 0 & -3.063782 & -.174263 & .415163 \\
\hline 4 & 15 & 0 & -1.184395 & 1.616855 & -.449843 \\
\hline 5 & 6 & 0 & -2.756241 & 1.237253 & -.023015 \\
\hline 6 & 1 & 0 & -1.099663 & 2.974501 & -.821470 \\
\hline 7 & 6 & 0 & -4.164672 & 1.535784 & .189304 \\
\hline 8 & 1 & 0 & -4.757895 & 2.436173 & .074640 \\
\hline 9 & 6 & 0 & -4.444801 & .241664 & .589208 \\
\hline
\end{tabular}




\begin{tabular}{|c|c|c|c|c|c|c|c|c|c|c|}
\hline \multicolumn{2}{|l|}{10} & \multicolumn{2}{|l|}{1} & 0 & \multicolumn{2}{|c|}{-5.341723} & -.27507 & \multicolumn{3}{|c|}{.911179} \\
\hline 11 & & 1 & & 0 & \multicolumn{2}{|c|}{5.693519} & 1.178442 & \multicolumn{3}{|c|}{1.184036} \\
\hline 12 & & 6 & & 0 & \multicolumn{2}{|c|}{4.791279} & 1.755291 & \multicolumn{3}{|c|}{.921232} \\
\hline 13 & & 7 & & 0 & \multicolumn{2}{|c|}{3.918852} & 1.083701 & \multicolumn{3}{|c|}{-.039417} \\
\hline 14 & & 1 & & 0 & \multicolumn{2}{|c|}{5.104474} & 2.717291 & \multicolumn{3}{|c|}{.506219} \\
\hline 15 & & 1 & & 0 & \multicolumn{2}{|c|}{4.231543} & 1.95500 & \multicolumn{3}{|c|}{1.841499} \\
\hline 16 & & 6 & & 0 & \multicolumn{2}{|c|}{3.484421} & -.23016 & \multicolumn{3}{|c|}{.391296} \\
\hline 17 & & 1 & & 0 & \multicolumn{2}{|c|}{4.399152} & 1.01446 & \multicolumn{3}{|c|}{-.934465} \\
\hline 18 & & 1 & & 0 & \multicolumn{2}{|c|}{3.093391} & -.146971 & & 12071 & \\
\hline 19 & & 1 & & 0 & 4.2 & 8170 & -.98976 & & 14991 & \\
\hline 20 & & 6 & & 0 & 1. & .7111 & -1.857173 & & 37118 & \\
\hline 21 & & 1 & & 0 & 1.3 & 2412 & -2.67925 & & 26238 & \\
\hline 22 & & 1 & & 0 & 1. & 6121 & -2.18506 & & 91967 & \\
\hline 23 & & 6 & & 0 & 2.3 & 5000 & -.76449 & & 23221 & \\
\hline 24 & & 1 & & 0 & 2. & 6143 & -.88696 & -1 & 65957 & \\
\hline 25 & & 1 & & 0 & 1. & 8637 & .300513 & & 74004 & \\
\hline 26 & & 78 & & 0 & & 1159 & -.33266 & & 89625 & \\
\hline & & & 1 & & & 2 & & & 3 & \\
\hline & & & A & & & A & & & A & \\
\hline Frequ & dencies & -- & 27.831 & & & 46.48 & & & 56.64 & \\
\hline Red. & masses & -- & $5.260^{\circ}$ & & & 4.95 & & & 4.45 & \\
\hline Frc & consts & -- & .002 & & & .00 & & & .00 & \\
\hline IR Ir & hten & -- & 2.900 & & & 2.73 & & & 1.40 & \\
\hline Atom & AN & X & Y & Z & $\mathrm{X}$ & Y & Z & $\mathrm{X}$ & Y & Z \\
\hline 1 & 15 & .00 & .02 & .03 & -.04 & .01 & -.03 & .06 & .05 & .20 \\
\hline 2 & 1 & .00 & .06 & .13 & -.12 & -.01 & -.16 & .09 & .05 & .23 \\
\hline 3 & 6 & .04 & .07 & .11 & -.01 & .04 & -.08 & .02 & .00 & .06 \\
\hline 4 & 15 & .03 & -.02 & -.09 & .10 & .03 & .13 & -.02 & .00 & -.04 \\
\hline 5 & 6 & .06 & .04 & .05 & .06 & .06 & .01 & -.02 & -.03 & -.06 \\
\hline 6 & 1 & .06 & -.03 & -.11 & .17 & .04 & .18 & -.07 & -.04 & -.19 \\
\hline 7 & 6 & .09 & .10 & .16 & .05 & .08 & -.08 & -.04 & -.07 & -.15 \\
\hline 8 & 1 & .11 & .12 & .20 & .08 & .10 & -.09 & -.08 & -.11 & -.28 \\
\hline 9 & 6 & .07 & .12 & .22 & -.01 & .07 & -.17 & .00 & -.04 & -.02 \\
\hline 10 & 1 & .08 & .16 & .31 & -.05 & .07 & -.27 & .01 & -.05 & .00 \\
\hline 11 & 1 & -.21 & .19 & .24 & -.15 & .27 & -.13 & -.09 & -.08 & .11 \\
\hline 12 & 6 & -.23 & .13 & .16 & -.23 & .15 & -.09 & -.12 & -.10 & .16 \\
\hline 13 & 7 & -.11 & .06 & .10 & -.17 & .07 & -.09 & -.05 & -.02 & .05 \\
\hline 14 & 1 & -.26 & .14 & .17 & -.37 & .21 & -.06 & -.16 & -.03 & .30 \\
\hline 15 & 1 & -.32 & .11 & .11 & -.23 & .05 & -.07 & -.16 & -.25 & .17 \\
\hline 16 & 6 & -.06 & .04 & .08 & .00 & .00 & -.12 & .00 & -.10 & -.13 \\
\hline 17 & 1 & -.04 & .08 & .14 & -.18 & .16 & -.10 & -.02 & .11 & .05 \\
\hline 18 & 1 & -.13 & .03 & .06 & .07 & $-.0 s$ & -.09 & .03 & -.25 & -.10 \\
\hline 19 & 1 & -.02 & .09 & .14 & .08 & .08 & -.22 & .03 & -.07 & -.25 \\
\hline 20 & 6 & .01 & -.03 & .02 & .01 & -.06 & -.06 & -.02 & -.03 & -.17 \\
\hline 21 & 1 & .04 & -.04 & .04 & -.02 & -.04 & -.07 & -.10 & .03 & -.22 \\
\hline 22 & 1 & -.02 & .00 & .04 & .04 & -.08 & -.07 & .04 & -.11 & -.21 \\
\hline 23 & 6 & .02 & -.03 & .03 & -.02 & -.04 & -.07 & -.02 & -.02 & -.15 \\
\hline 24 & 1 & .08 & -.06 & .06 & -.07 & -.01 & -.09 & -.04 & .03 & -.16 \\
\hline 25 & 1 & .00 & -.05 & -.06 & -.04 & -.03 & -.02 & -.01 & .00 & -.09 \\
\hline 26 & 78 & .01 & -.04 & -.06 & .02 & -.03 & .04 & .01 & .02 & .00 \\
\hline
\end{tabular}

thermodynamics:

Sum of electronic and zero-point Energies=

$-1169.196933$

Sum of electronic and thermal Enthalpies=

$-1169.180970$

Sum of electronic and thermal Free Energies=

$-1169.241114$

Total free energy in solution:

with all non electrostatic terms (a.u.) $=-1169.460690$

Optimized geometry, three lower frequencies, thermochemistry and PCM energy for $\mathbf{T S}_{\mathbf{1 6 a - 1 8 a}}$. 


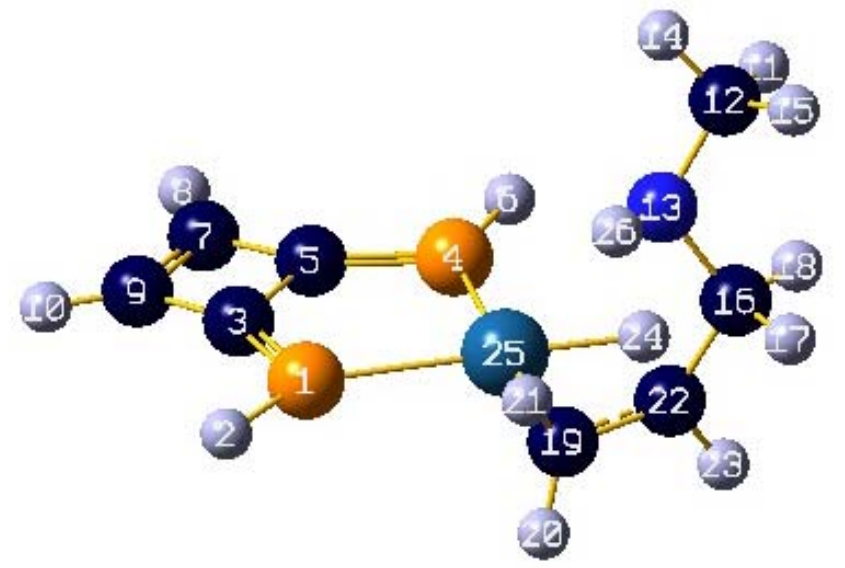

\begin{tabular}{|c|c|c|c|c|c|}
\hline \multirow{2}{*}{$\begin{array}{l}\text { Center } \\
\text { Number }\end{array}$} & \multirow{2}{*}{$\begin{array}{l}\text { Atomic } \\
\text { Number }\end{array}$} & \multirow{2}{*}{$\begin{array}{l}\text { Atomic } \\
\text { Type }\end{array}$} & \multicolumn{3}{|c|}{ Coordinates (Angstroms) } \\
\hline & & & $\mathrm{X}$ & $\mathrm{Y}$ & Z \\
\hline 1 & 15 & 0 & -1.512103 & -1.208020 & 0.891875 \\
\hline 2 & 1 & 0 & -1.762093 & -2.332445 & 1.703223 \\
\hline 3 & 6 & 0 & -2.883067 & -0.267116 & 0.725025 \\
\hline 4 & 15 & 0 & -1.340952 & 1.341271 & -0.848054 \\
\hline 5 & 6 & 0 & -2.800534 & 0.976795 & -0.122450 \\
\hline 6 & 1 & 0 & -1.427416 & 2.521041 & -1.611207 \\
\hline 7 & 6 & 0 & -4.204613 & 1.203670 & 0.188772 \\
\hline 8 & 1 & 0 & -4.911316 & 1.976985 & -0.090648 \\
\hline 9 & 6 & 0 & -4.280325 & 0.062455 & 0.964322 \\
\hline 10 & 1 & 0 & -5.069213 & -0.415386 & 1.533994 \\
\hline 11 & 1 & 0 & 3.750534 & 2.725340 & 0.758280 \\
\hline 12 & 6 & 0 & 3.916990 & 1.903562 & 1.462829 \\
\hline 13 & 7 & 0 & 3.109475 & 0.752242 & 1.063823 \\
\hline 14 & 1 & 0 & 3.590254 & 2.240433 & 2.450493 \\
\hline 15 & 1 & 0 & 4.999021 & 1.697387 & 1.504590 \\
\hline 16 & 6 & 0 & 3.484521 & 0.199052 & -0.217834 \\
\hline 17 & 1 & 0 & 4.441362 & -0.361485 & -0.206974 \\
\hline 18 & 1 & 0 & 3.627998 & 1.025514 & -0.922501 \\
\hline 19 & 6 & 0 & 1.758810 & -1.706419 & 0.032254 \\
\hline 20 & 1 & 0 & 1.476052 & -2.657058 & -0.418113 \\
\hline 21 & 1 & 0 & 1.972197 & -1.750061 & 1.099131 \\
\hline 22 & 6 & 0 & 2.461214 & -0.765688 & -0.778465 \\
\hline 23 & 1 & 0 & 2.618992 & -1.047297 & -1.820656 \\
\hline 24 & 1 & 0 & 1.399223 & 0.393473 & -1.264318 \\
\hline 25 & 78 & 0 & 0.236437 & -0.268724 & -0.335062 \\
\hline 26 & 1 & 0 & 3.163829 & 0.041725 & 1.788742 \\
\hline
\end{tabular}

\begin{tabular}{|c|c|c|c|c|c|c|c|c|c|c|}
\hline & & & 1 & & & 2 & & & 3 & \\
\hline & & & A & & & A & & & A & \\
\hline Frequ & dencies & -- & -522.40 & & & 28.43 & & & 43.39 & \\
\hline Red. & masses & -- & 1.40 & & & 5.11 & & & 4.80 & \\
\hline Frc $c$ & consts & -- & 0.22 & & & 0.00 & & & 0.00 & \\
\hline IR In & hten & -- & 20.96 & & & 3.37 & & & 0.26 & \\
\hline Atom & AN & X & Y & Z & $\mathrm{X}$ & $\mathrm{Y}$ & $\mathrm{Z}$ & X & $\mathrm{Y}$ & Z \\
\hline 1 & 15 & 0.01 & 0.02 & -0.02 & 0.02 & 0.01 & 0.00 & -0.07 & -0.09 & -0.13 \\
\hline 2 & 1 & 0.10 & -0.01 & -0.03 & 0.03 & 0.04 & 0.05 & -0.14 & -0.16 & -0.25 \\
\hline 3 & 6 & 0.00 & 0.00 & 0.00 & 0.05 & 0.07 & 0.09 & -0.03 & -0.02 & -0.08 \\
\hline 4 & 15 & 0.00 & 0.02 & -0.01 & 0.02 & -0.03 & -0.04 & 0.08 & 0.08 & 0.13 \\
\hline 5 & 6 & 0.01 & 0.00 & 0.00 & 0.05 & 0.05 & 0.06 & 0.05 & 0.07 & 0.06 \\
\hline 6 & 1 & -0.10 & 0.03 & 0.02 & 0.03 & -0.02 & -0.04 & 0.16 & 0.17 & 0.26 \\
\hline 7 & 6 & 0.00 & 0.00 & 0.00 & 0.09 & 0.13 & 0.16 & 0.05 & 0.09 & 0.04 \\
\hline 8 & 1 & 0.00 & 0.00 & 0.00 & 0.10 & 0.16 & 0.21 & 0.09 & 0.15 & 0.11 \\
\hline 9 & 6 & 0.00 & 0.00 & 0.00 & 0.09 & 0.14 & 0.18 & -0.03 & 0.00 & -0.10 \\
\hline 10 & 1 & 0.00 & 0.00 & 0.00 & 0.11 & 0.19 & 0.25 & -0.07 & -0.03 & -0.18 \\
\hline 11 & 1 & 0.00 & 0.00 & 0.00 & -0.29 & 0.08 & 0.11 & -0.06 & 0.08 & -0.32 \\
\hline 12 & 6 & 0.00 & 0.00 & 0.00 & -0.28 & 0.10 & 0.12 & -0.10 & 0.17 & -0.20 \\
\hline
\end{tabular}




$\begin{array}{rrrrrrrrrrr}13 & 7 & 0.00 & 0.00 & -0.01 & -0.16 & 0.04 & 0.08 & -0.07 & 0.12 & -0.10 \\ 14 & 1 & -0.01 & 0.00 & 0.00 & -0.36 & 0.08 & 0.10 & -0.16 & 0.30 & -0.27 \\ 15 & 1 & -0.01 & 0.00 & 0.01 & -0.26 & 0.18 & 0.19 & -0.10 & 0.18 & -0.11 \\ 16 & 6 & 0.05 & 0.00 & 0.00 & -0.05 & 0.06 & 0.10 & 0.00 & -0.04 & -0.02 \\ 17 & 1 & 0.01 & -0.05 & 0.05 & -0.02 & 0.10 & 0.17 & -0.02 & -0.06 & 0.10 \\ 18 & 1 & 0.12 & 0.01 & 0.03 & -0.04 & 0.06 & 0.11 & 0.06 & -0.12 & -0.10 \\ 19 & 6 & -0.05 & 0.05 & -0.01 & 0.02 & -0.02 & -0.01 & 0.00 & -0.04 & 0.04 \\ 20 & 1 & 0.06 & 0.01 & -0.01 & 0.06 & -0.02 & -0.03 & -0.01 & -0.05 & 0.05 \\ 21 & 1 & 0.05 & 0.03 & -0.03 & -0.02 & -0.02 & 0.00 & -0.01 & -0.02 & 0.04 \\ 22 & 6 & -0.04 & 0.11 & -0.08 & 0.03 & 0.01 & 0.03 & -0.01 & -0.06 & 0.02 \\ 23 & 1 & 0.17 & -0.09 & 0.01 & 0.11 & 0.02 & 0.04 & -0.03 & -0.07 & 0.02 \\ 24 & 1 & 0.69 & -0.62 & 0.14 & 0.01 & -0.05 & -0.06 & 0.01 & 0.01 & 0.06 \\ 25 & 78 & 0.00 & -0.01 & 0.01 & 0.01 & -0.04 & -0.05 & 0.01 & -0.02 & 0.03 \\ 26 & 1 & -0.02 & 0.00 & -0.01 & -0.16 & 0.04 & 0.08 & -0.12 & 0.21 & -0.01\end{array}$

thermodynamics:

Sum of electronic and zero-point Energies=

$-1169.193774$

Sum of electronic and thermal Enthalpies=

$-1169.177902$

Sum of electronic and thermal Free Energies=

$-1169.237870$

Total free energy in solution:

with all non electrostatic terms

$\left(\mathrm{a} \cdot \mathrm{u}_{.}\right)=-1169.453775$

Optimized geometry, three lower frequencies, thermochemistry and PCM energy for $\mathbf{1 7 a}$.

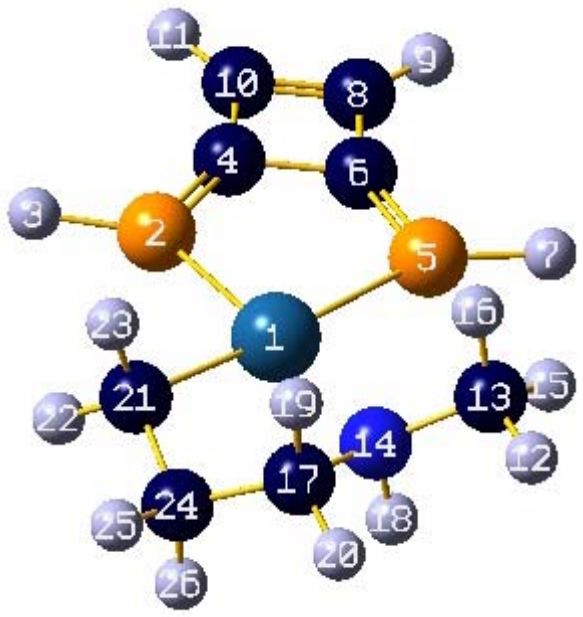

\begin{tabular}{|c|c|c|c|c|c|}
\hline \multirow{2}{*}{$\begin{array}{l}\text { Center } \\
\text { Number } \\
------\end{array}$} & \multirow{2}{*}{$\begin{array}{l}\text { Atomic } \\
\text { Number }\end{array}$} & \multirow{2}{*}{$\begin{array}{l}\text { Atomic } \\
\text { Type }\end{array}$} & \multicolumn{3}{|c|}{ Coordinates (Angstroms) } \\
\hline & & & $\mathrm{X}$ & $Y$ & Z \\
\hline 1 & 78 & 0 & .417887 & -.131853 & -.022640 \\
\hline 2 & 15 & 0 & -1.368416 & -1.490659 & .148588 \\
\hline 3 & 1 & 0 & -1.522735 & -2.881101 & .298085 \\
\hline 4 & 6 & 0 & -2.820577 & -.674777 & .086535 \\
\hline 5 & 15 & 0 & -1.295490 & 1.590333 & -.176346 \\
\hline 6 & 6 & 0 & -2.778482 & .820144 & -.073570 \\
\hline 7 & 1 & 0 & -1.545217 & 2.976103 & -.321955 \\
\hline 8 & 6 & 0 & -4.236874 & .797821 & -.049792 \\
\hline 9 & 1 & 0 & -4.993040 & 1.571458 & -.121491 \\
\hline 10 & 6 & 0 & -4.273695 & -.574535 & .097884 \\
\hline 11 & 1 & 0 & -5.068193 & -1.306545 & .188454 \\
\hline 12 & 1 & 0 & 3.233793 & 2.786556 & .341085 \\
\hline 13 & 6 & 0 & 2.251075 & 2.320725 & .480114 \\
\hline 14 & 7 & 0 & 2.202023 & 1.008959 & -.206013 \\
\hline 15 & 1 & 0 & 1.483880 & 2.979747 & .069242 \\
\hline
\end{tabular}




\begin{tabular}{|c|c|c|c|c|c|c|c|c|c|c|}
\hline \multicolumn{2}{|l|}{16} & \multicolumn{2}{|l|}{1} & 0 & \multicolumn{2}{|c|}{2.067895} & 2.175730 & \multicolumn{3}{|c|}{1.546412} \\
\hline 17 & & 6 & & 0 & \multicolumn{2}{|c|}{3.367289} & .142195 & \multicolumn{3}{|c|}{.160702} \\
\hline 18 & & 1 & & 0 & \multicolumn{2}{|c|}{2.271366} & 1.183572 & \multicolumn{3}{|c|}{-1.211814} \\
\hline 19 & & 1 & & 0 & \multicolumn{2}{|c|}{3.416969} & .121565 & \multicolumn{3}{|c|}{1.254857} \\
\hline 20 & & 1 & & 0 & \multicolumn{2}{|c|}{4.291019} & .596898 & \multicolumn{3}{|c|}{-.218554} \\
\hline 21 & & 6 & & 0 & \multicolumn{2}{|c|}{1.741939} & -1.716135 & \multicolumn{3}{|c|}{.124051} \\
\hline 22 & & 1 & & 0 & \multicolumn{2}{|c|}{1.396788} & -2.600437 & \multicolumn{3}{|c|}{-.422475} \\
\hline 23 & & 1 & & 0 & \multicolumn{2}{|c|}{1.795392} & -1.974039 & \multicolumn{3}{|c|}{1.191529} \\
\hline 24 & & 6 & & 0 & \multicolumn{2}{|c|}{3.100932} & -1.241551 & \multicolumn{3}{|c|}{-.391518} \\
\hline 25 & & 1 & & 0 & \multicolumn{2}{|c|}{3.900062} & -1.930724 & \multicolumn{3}{|c|}{-.086008} \\
\hline 26 & & 1 & & 0 & 3.1 & 1585 & -1.215393 & -1 & 89469 & \\
\hline & & & $1_{\mathrm{A}}$ & & & ${ }^{2} \mathrm{~A}$ & & & ${ }^{3}$ & \\
\hline Frequ & uencies & -- & 36.997 & & & 56.872 & & & 91.029 & \\
\hline Red. & masses & -- & 4.638 & & & 3.80 & & & 3.698 & \\
\hline Frc & consts & -- & .003 & & & .00 & & & .018 & \\
\hline IR $\operatorname{Ir}$ & nten & -- & 3.739 & & & $1.11 \xi$ & & & 1.520 & \\
\hline Atom & AN & $\mathrm{X}$ & Y & $\mathrm{Z}$ & $\mathrm{x}$ & Y & $\mathrm{Z}$ & $\mathrm{X}$ & Y & Z \\
\hline 1 & 78 & .00 & .01 & .06 & .00 & .00 & .01 & .00 & -.04 & -.01 \\
\hline 2 & 15 & .01 & .01 & .09 & -.01 & -.01 & -.08 & -.03 & .01 & .13 \\
\hline 3 & 1 & .01 & .00 & .04 & -.01 & -.03 & -.23 & -.09 & .01 & .14 \\
\hline 4 & 6 & .00 & -.02 & -.06 & .00 & -.01 & -.08 & .00 & .05 & .05 \\
\hline 5 & 15 & -.01 & .01 & .06 & .00 & .01 & .17 & .05 & .00 & .05 \\
\hline 6 & 6 & -.01 & -.02 & -.08 & .00 & .01 & .07 & .03 & .04 & -.03 \\
\hline 7 & 1 & -.02 & .00 & -.02 & .01 & .03 & .28 & .11 & .00 & -.08 \\
\hline 8 & 6 & -.01 & -.04 & -.23 & .00 & .00 & .01 & .03 & .07 & -.12 \\
\hline 9 & 1 & -.01 & -.05 & -.32 & .00 & .01 & .06 & .05 & .08 & -.23 \\
\hline 10 & 6 & .00 & -.04 & -.21 & -.01 & -.01 & -.13 & .00 & .08 & -.03 \\
\hline 11 & 1 & .00 & -.05 & -.28 & -.01 & -.02 & -.24 & -.02 & .10 & -.03 \\
\hline 12 & 1 & .06 & -.03 & -.11 & .03 & .04 & -.37 & -.16 & .08 & .04 \\
\hline 13 & 6 & .08 & -.03 & -.01 & .02 & .08 & -.30 & -.16 & .06 & -.03 \\
\hline 14 & 7 & -.01 & .00 & -.07 & .00 & -.03 & -.09 & -.05 & .03 & .01 \\
\hline 15 & 1 & .03 & -.01 & .12 & .04 & .03 & -.42 & -.13 & .02 & -.12 \\
\hline 16 & 1 & .21 & -.09 & .00 & .01 & .27 & -.28 & -.25 & .09 & -.04 \\
\hline 17 & 6 & .03 & -.02 & -.24 & -.02 & .01 & .05 & -.05 & .08 & .10 \\
\hline 18 & 1 & -.12 & .05 & -.07 & .02 & -.19 & -.11 & .00 & .00 & .00 \\
\hline 19 & 1 & .18 & -.04 & -.25 & -.09 & .07 & .06 & -.19 & .01 & .10 \\
\hline 20 & 1 & -.01 & -.03 & -.36 & .01 & -.01 & .09 & -.03 & .15 & .23 \\
\hline 21 & 6 & .00 & .01 & -.05 & -.01 & -.01 & .02 & .04 & -.03 & -.22 \\
\hline 22 & 1 & -.06 & -.01 & .02 & .02 & .00 & -.01 & .09 & .09 & -.43 \\
\hline 23 & 1 & .12 & .04 & -.04 & -.07 & -.03 & .02 & -.01 & -.25 & -.27 \\
\hline 24 & 6 & -.06 & -.01 & -.22 & .03 & -.02 & .10 & .07 & .11 & -.04 \\
\hline 25 & 1 & -.03 & -.03 & -.33 & .01 & .00 & .19 & .08 & .13 & -.02 \\
\hline 26 & 1 & -.20 & -.01 & -.22 & .11 & -.07 & .10 & .19 & .21 & -.03 \\
\hline
\end{tabular}

thermodynamics:

Sum of electronic and zero-point Energies=

$-1169.249379$

Sum of electronic and thermal Enthalpies=

$-1169.234206$

Sum of electronic and thermal Free Energies=

$-1169.291487$

Total free energy in solution:

with all non electrostatic terms

$(\mathrm{a} \cdot \mathrm{u} \cdot)=-1169.514675$

Optimized geometry, three lower frequencies, thermochemistry and PCM energy for 18a. 


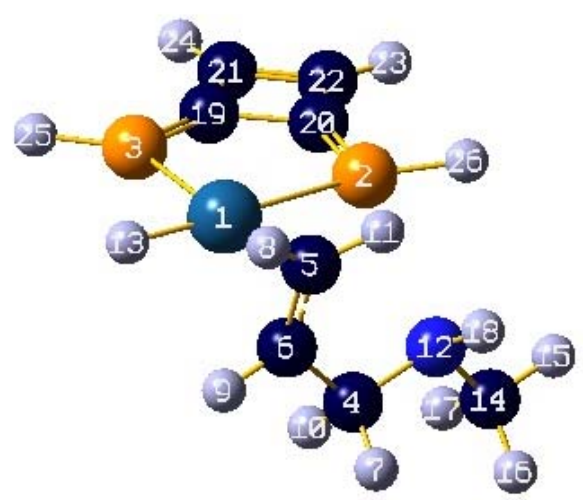

\begin{tabular}{|c|c|c|c|c|c|}
\hline \multirow{2}{*}{$\begin{array}{l}\text { Center } \\
\text { Number }\end{array}$} & \multirow{2}{*}{$\begin{array}{l}\text { Atomic } \\
\text { Number }\end{array}$} & \multirow{2}{*}{$\begin{array}{c}\text { Atomic } \\
\text { Type }\end{array}$} & \multicolumn{3}{|c|}{ Coordinates (Angstroms) } \\
\hline & & & $\mathrm{X}$ & $\mathrm{Y}$ & Z \\
\hline 1 & 78 & 0 & -.330238 & -.951730 & -.076684 \\
\hline 2 & 15 & 0 & .419542 & 1.235801 & -.816291 \\
\hline 3 & 15 & 0 & 1.843862 & -1.228375 & .604505 \\
\hline 4 & 6 & 0 & -2.506048 & 1.002528 & .917627 \\
\hline 5 & 6 & 0 & -2.324220 & -.790631 & -.956346 \\
\hline 6 & 6 & 0 & -2.418083 & -.404874 & .385055 \\
\hline 7 & 1 & 0 & -3.565194 & 1.166847 & 1.193622 \\
\hline 8 & 1 & 0 & -2.623503 & -1.794041 & -1.249259 \\
\hline 9 & 1 & 0 & -2.757332 & -1.152019 & 1.101942 \\
\hline 10 & 1 & 0 & -1.940955 & 1.057528 & 1.856273 \\
\hline 11 & 1 & 0 & -2.298546 & -.057673 & -1.759330 \\
\hline 12 & 7 & 0 & -2.008586 & 2.039041 & .025655 \\
\hline 13 & 1 & 0 & -.699862 & -2.376934 & .495382 \\
\hline 14 & 6 & 0 & -1.953942 & 3.359087 & .661305 \\
\hline 15 & 1 & 0 & -1.633954 & 4.102397 & -.074410 \\
\hline 16 & 1 & 0 & -2.922174 & 3.675732 & 1.076395 \\
\hline 17 & 1 & 0 & -1.218969 & 3.341048 & 1.472353 \\
\hline 18 & 1 & 0 & -2.605534 & 2.096979 & -.798452 \\
\hline 19 & 6 & 0 & 2.712073 & .175783 & .321943 \\
\hline 20 & 6 & 0 & 2.047708 & 1.319362 & -.411922 \\
\hline 21 & 6 & 0 & 3.927127 & .963496 & .359502 \\
\hline 22 & 6 & 0 & 3.317911 & 2.021499 & -.293369 \\
\hline 23 & 1 & 0 & 3.656250 & 3.001063 & -.614662 \\
\hline 24 & 1 & 0 & 4.928742 & .770237 & .725279 \\
\hline 25 & 1 & 0 & 2.592085 & -2.197118 & 1.296686 \\
\hline 26 & 1 & 0 & .141426 & 2.338690 & -1.646025 \\
\hline
\end{tabular}

\begin{tabular}{|c|c|c|c|c|c|c|c|c|c|c|}
\hline & & & 1 & & & 2 & & & 3 & \\
\hline & & & A & & & A & & & A & \\
\hline Frequ & dencies & -- & 28.40 & & & 55.65 & & & 91.705 & \\
\hline Red. & masses & -- & 4.86 & & & 4.43 & & & 5.045 & \\
\hline Frc $\mathrm{C}$ & onsts & -- & .00 & & & .00 & & & .025 & \\
\hline IR In & ten & -- & 9.95 & & & .48 & & & 4.499 & \\
\hline Atom & AN & $\mathrm{X}$ & Y & Z & $\mathrm{X}$ & Y & Z & $\mathrm{X}$ & $\mathrm{Y}$ & Z \\
\hline 1 & 78 & -.01 & -.03 & -.02 & .00 & .00 & .00 & -.01 & .01 & .06 \\
\hline 2 & 15 & -.05 & -.04 & -.08 & -.01 & -.02 & -.03 & -.06 & .03 & .07 \\
\hline 3 & 15 & -.01 & .03 & .01 & -.05 & .09 & .18 & .06 & -.06 & -.18 \\
\hline 4 & 6 & .14 & .05 & .05 & -.06 & -.07 & -.05 & -.11 & .00 & -.17 \\
\hline 5 & 6 & -.05 & -.01 & .08 & .06 & .03 & -.15 & .07 & -.04 & -.12 \\
\hline 6 & 6 & .05 & .04 & .07 & -.04 & -.04 & -.13 & -.06 & -.01 & -.14 \\
\hline 7 & 1 & .16 & .12 & .07 & -.07 & -.06 & -.13 & -.15 & .04 & -.35 \\
\hline 8 & 1 & -.11 & .00 & .13 & .09 & .04 & -.22 & .12 & -.05 & -.14 \\
\hline 9 & 1 & .06 & .07 & .12 & -.10 & -.08 & -.20 & -.13 & .00 & -.17 \\
\hline 10 & 1 & .17 & .04 & .03 & -.13 & -.15 & .00 & -.27 & -.02 & -.07 \\
\hline 11 & 1 & -.08 & -.04 & .05 & .11 & .07 & -.10 & .15 & -.05 & -.14 \\
\hline 12 & 7 & .18 & .02 & .02 & .04 & -.02 & .06 & .08 & -.01 & -.08 \\
\hline 13 & 1 & .00 & -.01 & .05 & -.02 & .00 & .00 & -.01 & .01 & .08 \\
\hline 14 & 6 & .28 & .02 & .00 & .15 & -.09 & .22 & .21 & -.05 & -.01 \\
\hline
\end{tabular}




\begin{tabular}{|c|c|c|c|c|c|c|c|c|c|c|}
\hline 15 & 1 & .31 & -.01 & -.02 & .20 & -.03 & .31 & .38 & -.06 & .05 \\
\hline 16 & 1 & .32 & .09 & .02 & .18 & -.07 & .27 & .21 & .07 & -.09 \\
\hline 17 & 1 & .31 & -.02 & -.02 & .16 & -.25 & .21 & .13 & -.20 & 5 \\
\hline 18 & 1 & .16 & .04 & .03 & .05 & .13 & .07 & .16 & .10 & -.13 \\
\hline 19 & 6 & -.07 & .09 & .07 & -.01 & .04 & .01 & .00 & .00 & -.07 \\
\hline 20 & 6 & -.08 & .04 & .00 & .00 & -.01 & -.07 & -.05 & .04 & .03 \\
\hline 21 & 6 & -.12 & .15 & .15 & .01 & .01 & -.09 & -.01 & .02 & -.06 \\
\hline 22 & 6 & -.13 & .11 & .09 & .02 & -.03 & -.17 & -.07 & .06 & .0 \\
\hline 23 & 1 & -.16 & .13 & .11 & .04 & -.07 & -.27 & -.11 & .10 & .1 \\
\hline 24 & 1 & -.13 & .21 & .23 & .02 & .01 & -.11 & .01 & .01 & -.12 \\
\hline 25 & 1 & -.02 & .11 & .12 & -.03 & .10 & .16 & .08 & -.05 & -.19 \\
\hline 26 & 1 & -.05 & -.11 & -.18 & -.03 & -.09 & -.12 & -.05 & .02 & .0 \\
\hline
\end{tabular}

thermodynamics:

Sum of electronic and zero-point Energies=

$-1169.205780$

Sum of electronic and thermal Enthalpies=

$-1169.189976$

Sum of electronic and thermal Free Energies=

$-1169.248942$

Total free energy in solution:

with all non electrostatic terms

$($ a.u. $)=-1169.464606$

Optimized geometry, three lower frequencies, thermochemistry and PCM energy for 19a.

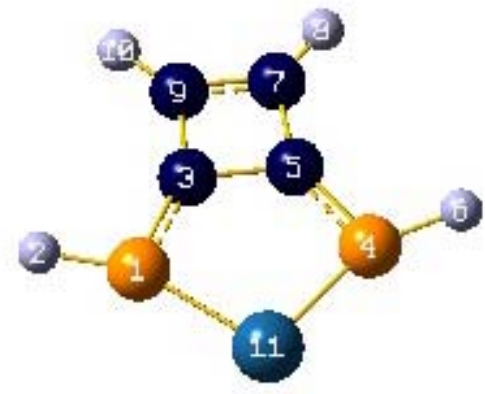

\begin{tabular}{|c|c|c|c|c|c|}
\hline \multirow{2}{*}{$\begin{array}{l}\text { Center } \\
\text { Number }\end{array}$} & \multirow{2}{*}{$\begin{array}{l}\text { Atomic } \\
\text { Number }\end{array}$} & \multirow{2}{*}{$\begin{array}{c}\text { Atomic } \\
\text { Type }\end{array}$} & \multicolumn{3}{|c|}{ Coordinates (Angstroms) } \\
\hline & & & $\mathrm{x}$ & $\mathrm{Y}$ & Z \\
\hline 1 & 15 & 0 & 0.418448 & 1.643193 & -0.000427 \\
\hline 2 & 1 & 0 & 0.867564 & 3.004115 & 0.000361 \\
\hline 3 & 6 & 0 & 1.860484 & 0.763380 & -0.000368 \\
\hline 4 & 15 & 0 & 0.418445 & -1.643191 & -0.000526 \\
\hline 5 & 6 & 0 & 1.860484 & -0.763384 & -0.000423 \\
\hline 6 & 1 & 0 & 0.867552 & -3.004116 & 0.000176 \\
\hline 7 & 6 & 0 & 3.306730 & -0.695033 & 0.000679 \\
\hline 8 & 1 & 0 & 4.087502 & -1.448369 & 0.001504 \\
\hline 9 & 6 & 0 & 3.306730 & 0.695030 & 0.000210 \\
\hline 10 & 1 & 0 & 4.087502 & 1.448366 & 0.000619 \\
\hline 11 & 78 & 0 & -1.082950 & 0.000000 & 0.000142 \\
\hline
\end{tabular}

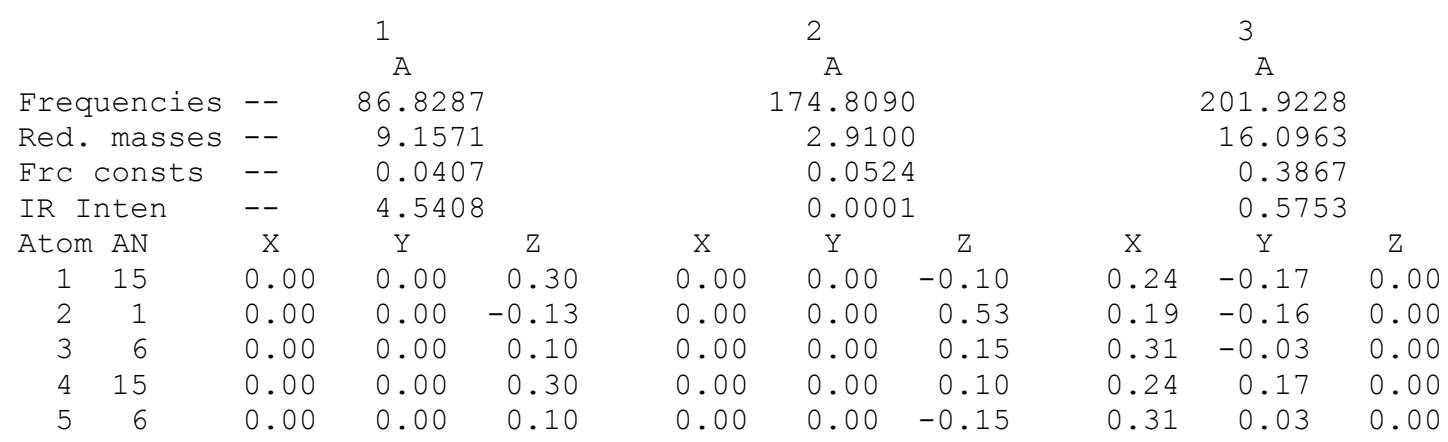




\begin{tabular}{|c|c|c|c|c|c|c|c|c|c|c|}
\hline 6 & 1 & 0.00 & 0.00 & -0.13 & 0.00 & 0.00 & -0.53 & 0.19 & 0.16 & 0.00 \\
\hline 7 & 6 & 0.00 & 0.00 & -0.27 & 0.00 & 0.00 & -0.19 & 0.35 & 0.01 & 0.00 \\
\hline 8 & 1 & 0.00 & 0.00 & -0.55 & 0.00 & 0.00 & -0.38 & 0.35 & 0.00 & 0.00 \\
\hline 9 & 6 & 0.00 & 0.00 & -0.27 & 0.00 & 0.00 & 0.19 & 0.35 & -0.01 & 0.00 \\
\hline 10 & 1 & 0.00 & 0.00 & -0.55 & 0.00 & 0.00 & 0.38 & 0.35 & 0.00 & 0.00 \\
\hline 11 & 78 & 0.00 & 0.00 & -0.07 & 0.00 & 0.00 & 0.00 & -0.16 & 0.00 & 0.00 \\
\hline
\end{tabular}

thermodynamics:

Sum of electronic and zero-point Energies=

$-956.421699$

Sum of electronic and thermal Enthalpies=

$-956.412784$

Sum of electronic and thermal Free Energies=

$-956.456250$

Total free energy in solution:

with all non electrostatic terms (a.u.) $=-956.486268$

Optimized geometry, three lower frequencies, thermochemistry and PCM energy for $\mathbf{T S}_{\mathbf{1 a - 2 0 a}}$.

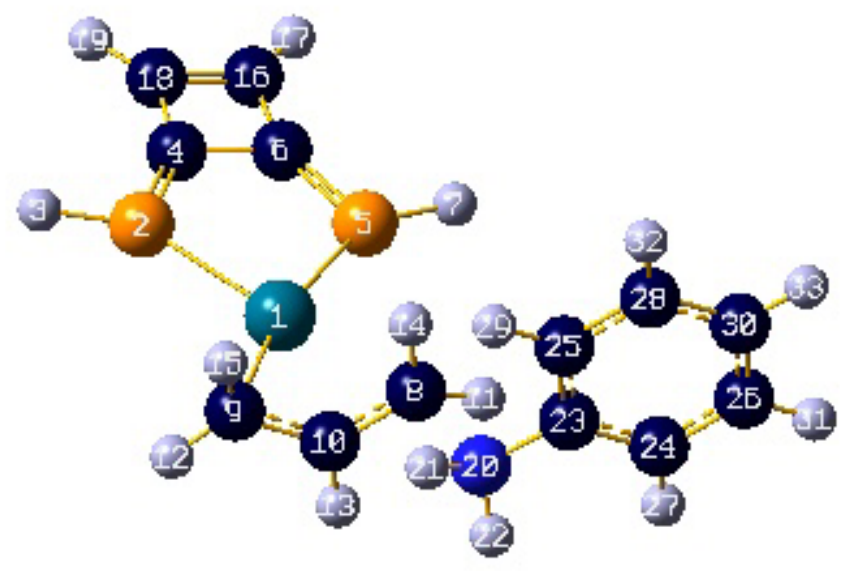

\begin{tabular}{|c|c|c|c|c|c|}
\hline \multirow{2}{*}{$\begin{array}{l}\text { Center } \\
\text { Number }\end{array}$} & \multirow{2}{*}{$\begin{array}{l}\text { Atomic } \\
\text { Number }\end{array}$} & \multirow{2}{*}{$\begin{array}{l}\text { Atomic } \\
\text { Type }\end{array}$} & \multicolumn{3}{|c|}{ Coordinates (Angstroms) } \\
\hline & & & $\mathrm{x}$ & $\mathrm{Y}$ & Z \\
\hline 1 & 46 & 0 & -1.199940 & -.693683 & -.184697 \\
\hline 2 & 15 & 0 & -3.331289 & -.916940 & .806222 \\
\hline 3 & 1 & 0 & -4.215538 & -1.788368 & 1.486994 \\
\hline 4 & 6 & 0 & -4.174078 & .507423 & .542975 \\
\hline 5 & 15 & 0 & -1.947475 & 1.512912 & -.755013 \\
\hline 6 & 6 & 0 & -3.517805 & 1.653753 & -.183965 \\
\hline 7 & 1 & 0 & -1.655432 & 2.750302 & -1.380693 \\
\hline 8 & 6 & 0 & 1.422513 & -.682039 & -.453712 \\
\hline 9 & 6 & 0 & -.151286 & -2.514529 & .113165 \\
\hline 10 & 6 & 0 & .584869 & -1.760441 & -.834993 \\
\hline 11 & 1 & 0 & 1.686497 & .080306 & -1.179511 \\
\hline 12 & 1 & 0 & -.686354 & -3.402870 & -.213219 \\
\hline 13 & 1 & 0 & .510724 & -2.008102 & -1.892919 \\
\hline 14 & 1 & 0 & 1.486022 & -.379694 & .587996 \\
\hline 15 & 1 & 0 & .137950 & -2.507817 & 1.164721 \\
\hline 16 & 6 & 0 & -4.761712 & 2.393344 & -.007980 \\
\hline 17 & 1 & 0 & -5.082035 & 3.388130 & -.297556 \\
\hline 18 & 6 & 0 & -5.362965 & 1.343176 & .656531 \\
\hline 19 & 1 & 0 & -6.341957 & 1.186466 & 1.095925 \\
\hline 20 & 7 & 0 & 3.414675 & -1.324413 & -.405734 \\
\hline 21 & 1 & 0 & 3.398973 & -2.097502 & .256104 \\
\hline 22 & 1 & 0 & 3.580464 & -1.699873 & -1.337230 \\
\hline 23 & 6 & 0 & 4.330939 & -.294055 & -.046929 \\
\hline 24 & 6 & 0 & 4.887775 & .517544 & -1.040203 \\
\hline 25 & 6 & 0 & 4.605423 & -.047499 & 1.301885 \\
\hline 26 & 6 & 0 & 5.728331 & 1.569110 & -.679158 \\
\hline
\end{tabular}




$\begin{array}{rrrrrr}27 & 1 & 0 & 4.679021 & .322360 & -2.090679 \\ 28 & 6 & 0 & 5.447383 & 1.006673 & 1.651552 \\ 29 & 1 & 0 & 4.176623 & -.682462 & 2.075223 \\ 30 & 6 & 0 & 6.009739 & 1.818289 & .665019 \\ 31 & 1 & 0 & 6.169143 & 2.190611 & -1.453747 \\ 32 & 1 & 0 & 5.668391 & 1.188687 & 2.699694 \\ 33 & 1 & 0 & 6.668746 & 2.636072 & .941838\end{array}$

\begin{tabular}{|c|c|c|c|c|c|c|c|c|c|c|}
\hline & \multicolumn{3}{|c|}{1} & \multicolumn{3}{|c|}{2} & \multicolumn{3}{|c|}{3} \\
\hline & & \multicolumn{3}{|c|}{ A } & \multicolumn{3}{|c|}{ A } & \multicolumn{3}{|c|}{$\mathrm{A}$} \\
\hline \multirow{2}{*}{\multicolumn{2}{|c|}{ Frequencies }} & -- & \multicolumn{2}{|c|}{$\begin{array}{c}A \\
-217.2066\end{array}$} & \multicolumn{3}{|c|}{12.7375} & \multicolumn{3}{|c|}{16.8181} \\
\hline & & -- & \multicolumn{2}{|c|}{6.9967} & \multicolumn{3}{|c|}{4.7760} & \multicolumn{3}{|c|}{6.5887} \\
\hline Frc C & consts & -- & \multirow{2}{*}{\multicolumn{2}{|c|}{$\begin{array}{r}.1945 \\
95085\end{array}$}} & \multicolumn{3}{|c|}{.0005} & \multicolumn{3}{|c|}{.0011} \\
\hline IR In & ten & -- & & & \multicolumn{3}{|c|}{.9094} & \multicolumn{3}{|c|}{1.7788} \\
\hline Atom & AN & X & Y & Z & $\mathrm{X}$ & $\mathrm{Y}$ & Z & $\mathrm{X}$ & Y & Z \\
\hline 1 & 46 & -.02 & -.02 & -.01 & .01 & .01 & .01 & .00 & -.07 & -.06 \\
\hline 2 & 15 & .00 & .01 & .02 & .07 & .06 & .15 & .02 & .03 & .00 \\
\hline 3 & 1 & -.04 & .05 & .04 & .12 & .10 & .26 & .02 & .10 & .10 \\
\hline 4 & 6 & .00 & .01 & .01 & .02 & .02 & .06 & .10 & .09 & .09 \\
\hline 5 & 15 & .00 & -.01 & -.01 & -.06 & -.05 & -.14 & .06 & -.05 & -.08 \\
\hline 6 & 6 & .00 & .00 & .00 & -.04 & -.03 & -.07 & .12 & .05 & .04 \\
\hline 7 & 1 & -.08 & .01 & -.03 & -.12 & -.10 & -.26 & .11 & -.06 & -.08 \\
\hline 8 & 6 & .55 & -.07 & .08 & -.01 & .02 & -.13 & -.01 & -.08 & -.03 \\
\hline 9 & 6 & .13 & .08 & -.03 & .05 & .05 & .08 & -.01 & -.06 & .01 \\
\hline 10 & 6 & .13 & .17 & .04 & -.01 & -.01 & -.01 & .00 & -.09 & -.01 \\
\hline 11 & 1 & .24 & .11 & .15 & -.05 & -.03 & -.20 & -.01 & -.10 & -.06 \\
\hline 12 & 1 & .15 & .10 & -.10 & .04 & .02 & .17 & -.01 & -.07 & .04 \\
\hline 13 & 1 & .13 & .21 & .02 & -.07 & -.09 & .01 & .02 & -.13 & .00 \\
\hline 14 & 1 & .23 & .05 & .07 & .05 & .09 & -.15 & -.03 & -.04 & -.04 \\
\hline 15 & 1 & .10 & .01 & -.02 & .11 & .12 & .06 & -.02 & -.03 & .02 \\
\hline 16 & 6 & .00 & .00 & -.01 & -.05 & -.05 & -.08 & .19 & .14 & .15 \\
\hline 17 & 1 & .00 & .00 & -.01 & -.08 & -.08 & -.16 & .24 & .17 & .19 \\
\hline 18 & 6 & .00 & .01 & .00 & .01 & .00 & .04 & .17 & .18 & .20 \\
\hline 19 & 1 & -.01 & .01 & -.01 & .03 & .01 & .10 & .20 & .25 & .28 \\
\hline 20 & 7 & -.29 & .10 & .01 & .00 & .03 & -.18 & -.01 & -.03 & .03 \\
\hline 21 & 1 & -.25 & .07 & -.02 & -.01 & -.02 & -.24 & .00 & -.02 & .04 \\
\hline 22 & 1 & -.29 & .08 & .01 & .02 & .11 & -.20 & .03 & -.04 & .04 \\
\hline 23 & 6 & -.12 & .04 & .00 & -.01 & .00 & -.07 & -.06 & .02 & .03 \\
\hline 24 & 6 & -.08 & -.02 & -.01 & .02 & .09 & .01 & -.08 & .03 & .03 \\
\hline 25 & 6 & -.07 & -.01 & .00 & -.04 & -.11 & -.04 & -.11 & .06 & .03 \\
\hline 26 & 6 & -.02 & -.05 & -.02 & .01 & .06 & .12 & -.14 & .08 & .03 \\
\hline 27 & 1 & -.11 & .01 & -.01 & .04 & .18 & -.01 & -.04 & .00 & .03 \\
\hline 28 & 6 & -.02 & -.05 & -.01 & -.04 & -.14 & .07 & -.17 & .11 & .03 \\
\hline 29 & 1 & -.10 & .02 & .00 & -.05 & -.18 & -.11 & -.10 & .05 & .03 \\
\hline 30 & 6 & -.01 & -.06 & -.02 & -.02 & -.06 & .15 & -.19 & .12 & .03 \\
\hline 31 & 1 & .00 & -.07 & -.02 & .03 & .12 & .19 & -.16 & .09 & .03 \\
\hline 32 & 1 & .00 & -.06 & -.01 & -.07 & -.23 & .09 & -.21 & .14 & .04 \\
\hline 33 & 1 & .02 & -.08 & -.02 & -.03 & -.08 & .23 & -.24 & .16 & .03 \\
\hline
\end{tabular}

thermodynamics:

Sum of electronic and zero-point Energies=

$-1368.389059$

Sum of electronic and thermal Enthalpies=

$-1368.369417$

Sum of electronic and thermal Free Energies=

$-1368.440632$

Total free energy in solution:

with all non electrostatic terms

$(\mathrm{a} \cdot \mathrm{u} \cdot)=-1368.697125$

Optimized geometry, three lower frequencies, thermochemistry and PCM energy for 20a. 


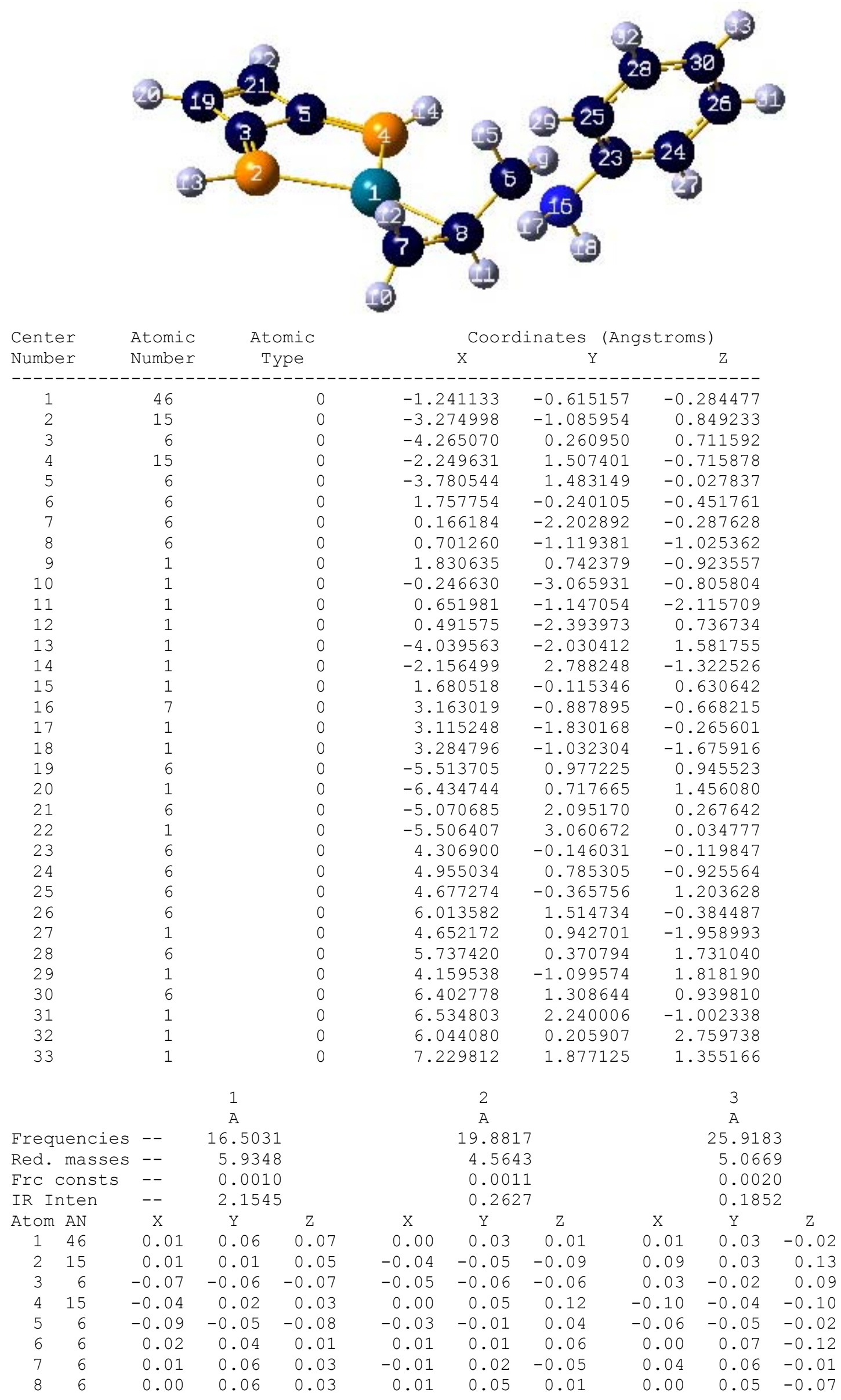




$\begin{array}{rrrrrrrrrrr}9 & 1 & 0.01 & 0.05 & 0.03 & 0.04 & 0.03 & 0.09 & 0.01 & 0.05 & -0.16 \\ 10 & 1 & 0.00 & 0.06 & 0.03 & -0.01 & 0.05 & -0.09 & 0.04 & 0.04 & 0.03 \\ 11 & 1 & -0.03 & 0.07 & 0.03 & 0.02 & 0.11 & 0.01 & -0.03 & 0.01 & -0.07 \\ 12 & 1 & 0.04 & 0.05 & 0.02 & -0.03 & -0.03 & -0.05 & 0.08 & 0.10 & -0.02 \\ 13 & 1 & 0.01 & -0.02 & 0.01 & -0.06 & -0.11 & -0.19 & 0.17 & 0.05 & 0.23 \\ 14 & 1 & -0.09 & -0.01 & -0.03 & 0.01 & 0.09 & 0.19 & -0.19 & -0.08 & -0.20 \\ 15 & 1 & 0.06 & 0.02 & 0.01 & 0.00 & -0.02 & 0.06 & 0.01 & 0.12 & -0.12 \\ 16 & 7 & 0.01 & 0.03 & -0.05 & 0.00 & -0.01 & 0.06 & 0.00 & 0.05 & -0.09 \\ 17 & 1 & 0.00 & 0.02 & -0.09 & -0.01 & 0.00 & 0.09 & -0.04 & 0.04 & -0.12 \\ 18 & 1 & -0.02 & 0.08 & -0.06 & -0.02 & -0.04 & 0.06 & 0.03 & 0.08 & -0.09 \\ 19 & 6 & -0.14 & -0.13 & -0.21 & -0.08 & -0.09 & -0.08 & 0.01 & -0.06 & 0.11 \\ 20 & 1 & -0.16 & -0.18 & -0.27 & -0.10 & -0.14 & -0.15 & 0.05 & -0.06 & 0.18 \\ 21 & 6 & -0.16 & -0.13 & -0.22 & -0.06 & -0.05 & 0.01 & -0.07 & -0.09 & 0.00 \\ 22 & 1 & -0.21 & -0.17 & -0.29 & -0.06 & -0.04 & 0.04 & -0.13 & -0.12 & -0.05 \\ 23 & 6 & 0.03 & -0.01 & -0.05 & 0.02 & -0.01 & 0.02 & 0.00 & 0.00 & -0.03 \\ 24 & 6 & -0.01 & 0.07 & 0.01 & 0.13 & -0.17 & -0.08 & 0.14 & -0.08 & -0.01 \\ 25 & 6 & 0.11 & -0.14 & -0.09 & -0.05 & 0.14 & 0.07 & -0.14 & 0.04 & 0.02 \\ 26 & 6 & 0.02 & 0.02 & 0.02 & 0.16 & -0.17 & -0.13 & 0.14 & -0.13 & 0.06 \\ 27 & 1 & -0.07 & 0.17 & 0.04 & 0.19 & -0.29 & -0.11 & 0.25 & -0.10 & -0.04 \\ 28 & 6 & 0.14 & -0.19 & -0.08 & -0.02 & 0.14 & 0.01 & -0.14 & -0.01 & 0.09 \\ 29 & 1 & 0.15 & -0.20 & -0.13 & -0.13 & 0.26 & 0.14 & -0.25 & 0.10 & 0.00 \\ 30 & 6 & 0.09 & -0.11 & -0.02 & 0.08 & -0.02 & -0.09 & 0.00 & -0.10 & 0.11 \\ 31 & 1 & -0.02 & 0.08 & 0.06 & 0.24 & -0.29 & -0.21 & 0.25 & -0.19 & 0.08 \\ 32 & 1 & 0.20 & -0.29 & -0.11 & -0.08 & 0.26 & 0.05 & -0.25 & 0.01 & 0.12 \\ 33 & 1 & 0.12 & -0.15 & -0.02 & 0.10 & -0.02 & -0.13 & 0.00 & -0.14 & 0.16\end{array}$

thermodynamics:

Sum of electronic and zero-point Energies=

$-1368.397145$

Sum of electronic and thermal Enthalpies=

$-1368.377660$

Sum of electronic and thermal Free Energies=

$-1368.448081$

Total free energy in solution:

with all non electrostatic terms

$(\mathrm{a} \cdot \mathrm{u})=$.

Optimized geometry, three lower frequencies, thermochemistry and PCM energy for 21a. 


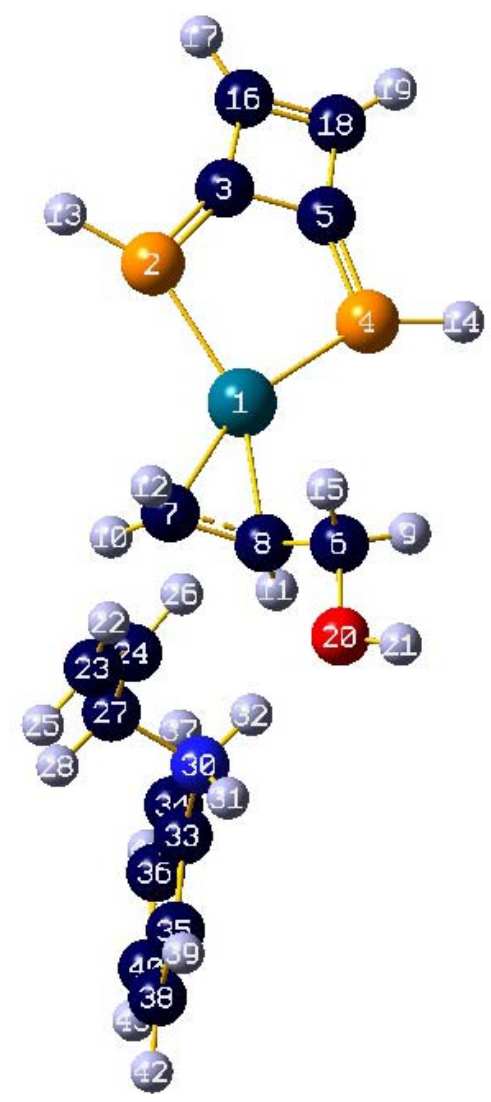

\begin{tabular}{|c|c|c|c|c|c|}
\hline Center & Atomic & Atomic & $\mathrm{COO}$ & inates (An & troms) \\
\hline Number & Number & Type & X & Y & Z \\
\hline 1 & 46 & 0 & 1.868592 & -.030566 & -.072472 \\
\hline 2 & 15 & 0 & 3.635606 & .606249 & -1.533645 \\
\hline 3 & 6 & 0 & 5.077104 & .102770 & -.836274 \\
\hline 4 & 15 & 0 & 3.614438 & -.914959 & 1.278460 \\
\hline 5 & 6 & 0 & 5.066478 & -.615943 & .490315 \\
\hline 6 & 6 & 0 & -.420576 & .491840 & 1.852509 \\
\hline 7 & 6 & 0 & -.115547 & .487013 & -.674040 \\
\hline 8 & 6 & 0 & -.185861 & -.205489 & .551489 \\
\hline 9 & 1 & 0 & .042861 & -.050830 & 2.682284 \\
\hline 10 & 1 & 0 & -.327696 & -.019934 & -1.614655 \\
\hline 11 & 1 & 0 & -.433714 & -1.268320 & .549300 \\
\hline 12 & 1 & 0 & -.194940 & 1.574618 & -.701486 \\
\hline 13 & 1 & 0 & 4.036559 & 1.227738 & -2.747381 \\
\hline 14 & 1 & 0 & 4.002313 & -1.598875 & 2.462991 \\
\hline 15 & 1 & 0 & -.032589 & 1.516530 & 1.832632 \\
\hline 16 & 6 & 0 & 6.529252 & -.035147 & -.825843 \\
\hline 17 & 1 & 0 & 7.309470 & .267129 & -1.516057 \\
\hline 18 & 6 & 0 & 6.519287 & -.693544 & .387372 \\
\hline 19 & 1 & 0 & 7.288543 & -1.112995 & 1.026725 \\
\hline 20 & 8 & 0 & -1.863365 & .551961 & 2.121181 \\
\hline 21 & 1 & 0 & -2.000192 & .723829 & 3.065562 \\
\hline 22 & 1 & 0 & -3.847294 & 5.134529 & .068960 \\
\hline 23 & 6 & 0 & -4.144622 & 4.164996 & -.321039 \\
\hline 24 & 6 & 0 & -3.405399 & 3.073717 & -.105528 \\
\hline 25 & 1 & 0 & -5.059696 & 4.136163 & -.910312 \\
\hline 26 & 1 & 0 & -2.483607 & 3.139345 & .471353 \\
\hline 27 & 6 & 0 & -3.762073 & 1.727468 & -.651473 \\
\hline 28 & 1 & 0 & -4.652481 & 1.760774 & -1.284593 \\
\hline 29 & 1 & 0 & -2.940830 & 1.273112 & -1.213625 \\
\hline 30 & 7 & 0 & -4.064418 & .758695 & .480665 \\
\hline 31 & 1 & 0 & -4.739237 & 1.213665 & 1.103244 \\
\hline 32 & 1 & 0 & -3.189521 & .630692 & 1.060693 \\
\hline 33 & 6 & 0 & -4.609578 & -.535869 & .045781 \\
\hline
\end{tabular}




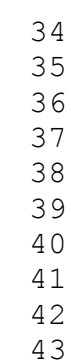

34
35
36
37
38
39
40
41
42
43

$+2$

Frequencies --

Red. masses --

Frc consts --

$\begin{array}{lr}\text { IR Inten } & -- \\ \text { Atom AN } & \mathrm{X}\end{array}$

$\begin{array}{rrr}1 & 46 & .00 \\ 2 & 15 & -.01 \\ 3 & 6 & .01\end{array}$

$\begin{array}{rrr}3 & 6 & .01 \\ 4 & 15 & .03\end{array}$

$\begin{array}{lll}5 & 6 & .03 \\ 6 & 6 & .00\end{array}$

$\begin{array}{rrr}6 & 6 & .00 \\ 7 & 6 & -.01\end{array}$

$8 \quad 6 \quad .01$

$\begin{array}{rrr}9 & 1 & .02 \\ 10 & 1 & .00\end{array}$

$\begin{array}{lll}11 & 1 & .02\end{array}$

$121-.02$

131

141

$15-1 \quad-.01$

$16 \quad 6 \quad .02$

$\begin{array}{lll}16 & 6 & .02 \\ 17 & 1 & .01\end{array}$

$18 \quad 6 \quad .04$

191.06

$\begin{array}{lll}19 & 8 & .00\end{array}$

$21 \quad 1 \quad .01$

$22 \quad 1 \quad .24$

$\begin{array}{lll}23 & 6 & .19 \\ 24 & 6 & .12\end{array}$

2511.18

$\begin{array}{lll}25 & 1 & .18 \\ 26 & 1 & .13\end{array}$

$\begin{array}{lll}27 & 6 & .05 \\ 28 & 1 & .05\end{array}$

$\begin{array}{lll}28 & 1 & .05 \\ 29 & 1 & .02\end{array}$

$\begin{array}{lll}30 & 7 & .01\end{array}$

$\begin{array}{lll}31 & 1 & .03\end{array}$

$\begin{array}{rrr}32 & 1 & .00 \\ 33 & 6 & -.05\end{array}$

$346-.08$

$356-.06$

$36 \quad 6 \quad-.14$

$37 \quad 1 \quad-.07$

$38 \quad 6 \quad-.12$

$391-.03$

$40 \quad 6 \quad-.15$

$41 \quad 1-.17$

$42 \quad 1 \quad-.13$

$431-.19$
1
A

9.8276

5.7502

.0003

.8611

$\mathrm{Y}$

$-.06-.02$

$.00-.01$

.12

.02

.13

$-.10$

$-.10$

$-.10$

$-.09$

$-.11$

$-.10$

$-.10$

.04

.07

$-.09$

.24

.30

.25

.31

$-.12$

$-.16$

$-.01$

.00

$-.04$

.05

$-.08$

$-.02$

.02

$-.07$

.00

.04

-.03
.02

$-.03$

.10

$-.01$

$-.09$

.12

.15

.06

$-.06$

.18
.08

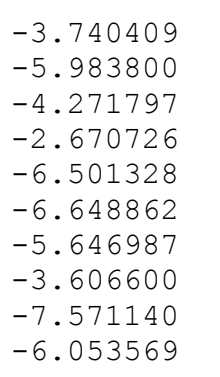

-1.501091
-.745068
-2.713362
-1.314602
-1.963266
.021639
-2.944111
-3.479485
-2.142635
-3.891444

$-.452575$

.116395

$-.892103$

$-.488629$

$-.324062$

.510153

$-.829012$

$-1.279953$

$-.269891$

$-1.171421$

thermodynamics:

Sum of electronic and zero-point Energies=

2
A

15.0730

6.0420

.0008

2.0378

X

.02

.04

.02

.00

.00

$-.02$

.02

.01

$-.03$

.05

.02

.01

.05

$-.02$

$-.04$

.01

.01

$-.01$

$-.02$

$-.03$

$-.05$

.15

.12

.08

.13

.07

.04

.05

.04

.00

.01

$-.01$

$-.04$

$-.07$

$-.05$

$-.11$

$-.06$

$-.09$

$-.03$

$-.12$

$-.13$

$-.10$

$-.15$
Y

.08

.17

.02

$-.07$

-.09
-.08

-.08
.10

.01

$-.13$

$.16-.02$

$.01-.11$

$.10 \quad .10$

.23

$-.21$

$-.07$

$-.08$

$-.08$

$-.19$

$-.30$

$-.12$

$-.19$

$-.06$

$-.06$

$-.08$

$-.05$

$-.09$

-.09
-.08

$-.13$

$-.04$

.01
-.05

$-.03$

$-.09$

.02

$-.08$

$-.13$

.03

.07

$-.03$

$-.12$

.07

$-.02$
$-.03$

.12

$-.12$

.10

$-.04$

$-.03$

$-.09$

$-.15$

.00

.01

$-.18$

$-.15$

$-.10$

$-.17$

$-.08$

$-.06$

$-.07$

$-.03$

$-.05$

$-.02$

.01

.07

$-.02$

.10

.08

.02

$-.06$

.08

.15

.00

.11
3

8.9411

5.4427

.0012

.8471

$\mathrm{Y}$

X
.00

.02

Z

$\begin{array}{rrr}.007 & -.05 & -.06 \\ -.05 & -.05\end{array}$

$-.04-.06 \quad-.11$

$.06 \quad .14 \quad .06$

$.02 \quad .04-.05$

$\begin{array}{rrr}.03 & .02 & .05\end{array}$

$-.03-.10 \quad .05$

$.01-.04 \quad .09$

$.00 \quad .08 \quad .08$

$\begin{array}{lll}-.01 & -.15 & .08\end{array}$

$.05-.05 \quad .14$

$-.07$

$-.13$

.11

$-.08$

$-.05$

$-.08$

.01

.03

$-.03$

$-.04$

.11

.09

.06

.10

.05

.03

.04

.03

$-.01$

-.02
-.02

.00

.02
-.02

.03

.03

$-.01$

$-.04$

.01

.04

$-.02$

.02

$-.10$

.00

$-.18-.13$

$.20 \quad .08$

$.03 \quad .00$
-.12 
Total free energy in solution:

with all non electrostatic terms (a.u.) = $\quad-1561.762535$

Optimized geometry, three lower frequencies, thermochemistry and PCM energy for $\mathbf{T S}_{\mathbf{2 1 a - 1 a}}$.

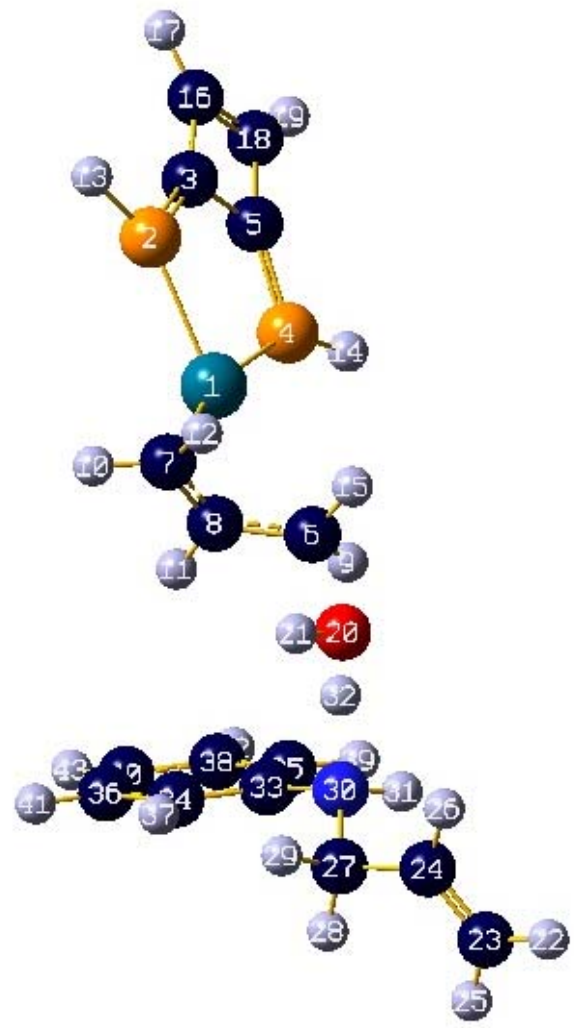

\begin{tabular}{|c|c|c|c|c|c|}
\hline \multirow{2}{*}{$\begin{array}{l}\text { Center } \\
\text { Number }\end{array}$} & \multirow{2}{*}{$\begin{array}{l}\text { Atomic } \\
\text { Number }\end{array}$} & \multirow{2}{*}{$\begin{array}{c}\text { Atomic } \\
\text { Type }\end{array}$} & \multicolumn{3}{|c|}{ Coordinates (Angstroms) } \\
\hline & & & $\mathrm{X}$ & $\mathrm{Y}$ & $\mathrm{Z}$ \\
\hline 1 & 46 & 0 & -1.873432 & -0.449185 & -0.414415 \\
\hline 2 & 15 & 0 & -4.108398 & -0.692874 & -1.141311 \\
\hline 3 & 6 & 0 & -5.108998 & -0.043433 & 0.036298 \\
\hline 4 & 15 & 0 & -2.855927 & 0.565499 & 1.522803 \\
\hline 5 & 6 & 0 & -4.517544 & 0.544884 & 1.292745 \\
\hline 6 & 6 & 0 & 0.657487 & -1.251472 & 0.314312 \\
\hline 7 & 6 & 0 & -0.564385 & -1.276355 & -1.854547 \\
\hline 8 & 6 & 0 & 0.241295 & -0.612494 & -0.894826 \\
\hline 9 & 1 & 0 & 0.949607 & -0.628297 & 1.153498 \\
\hline 10 & 1 & 0 & -0.684420 & -0.841439 & -2.844133 \\
\hline 11 & 1 & 0 & 0.650504 & 0.372217 & -1.113619 \\
\hline 12 & 1 & 0 & -0.699725 & -2.357659 & -1.805715 \\
\hline 13 & 1 & 0 & -4.965998 & -1.148249 & -2.173315 \\
\hline 14 & 1 & 0 & -2.666332 & 1.180851 & 2.786694 \\
\hline 15 & 1 & 0 & 0.209300 & -2.202418 & 0.586951 \\
\hline 16 & 6 & 0 & -6.421397 & 0.303242 & 0.568498 \\
\hline 17 & 1 & 0 & -7.431388 & 0.179927 & 0.193173 \\
\hline 18 & 6 & 0 & -5.880032 & 0.841956 & 1.718396 \\
\hline 19 & 1 & 0 & -6.296825 & 1.309698 & 2.603615 \\
\hline 20 & 8 & 0 & 2.340708 & -1.984237 & 0.113178 \\
\hline 21 & 1 & 0 & 2.387338 & -2.274047 & -0.813747 \\
\hline 22 & 1 & 0 & 7.413402 & -3.006237 & 1.994455 \\
\hline 23 & 6 & 0 & 7.134359 & -2.097709 & 1.467551 \\
\hline 24 & 6 & 0 & 5.990960 & -2.012511 & 0.783829 \\
\hline 25 & 1 & 0 & 7.839749 & -1.269708 & 1.513686 \\
\hline 26 & 1 & 0 & 5.312798 & -2.866445 & 0.754154 \\
\hline
\end{tabular}




$\begin{array}{ll}27 & 6 \\ 28 & 1 \\ 29 & 1 \\ 30 & 7 \\ 31 & 1 \\ 32 & 1 \\ 33 & 6 \\ 34 & 6 \\ 35 & 6 \\ 36 & 6 \\ 37 & 1 \\ 38 & 6 \\ 39 & 1 \\ 40 & 6 \\ 41 & 1 \\ 42 & 1 \\ 43 & 1\end{array}$

$\begin{array}{rrr}5.568886 & -0.793029 & 0.019716 \\ 6.340770 & -0.014215 & 0.066535 \\ 5.415794 & -1.043783 & -1.036640 \\ 4.282086 & -0.269060 & 0.556853 \\ 4.362598 & -0.239042 & 1.573313 \\ 3.118833 & -1.286031 & 0.270911 \\ 3.859067 & 1.018185 & 0.081594 \\ 3.882721 & 1.325841 & -1.282921 \\ 3.360862 & 1.956494 & 0.992354 \\ 3.415751 & 2.564989 & -1.723796 \\ 4.271561 & 0.614234 & -2.006225 \\ 2.888793 & 3.188956 & 0.542510 \\ 3.356518 & 1.731404 & 2.058090 \\ 2.914504 & 3.499635 & -0.817594 \\ 3.450622 & 2.798789 & -2.784732 \\ 2.515417 & 3.913027 & 1.262015 \\ 2.558399 & 4.464823 & -1.166373\end{array}$

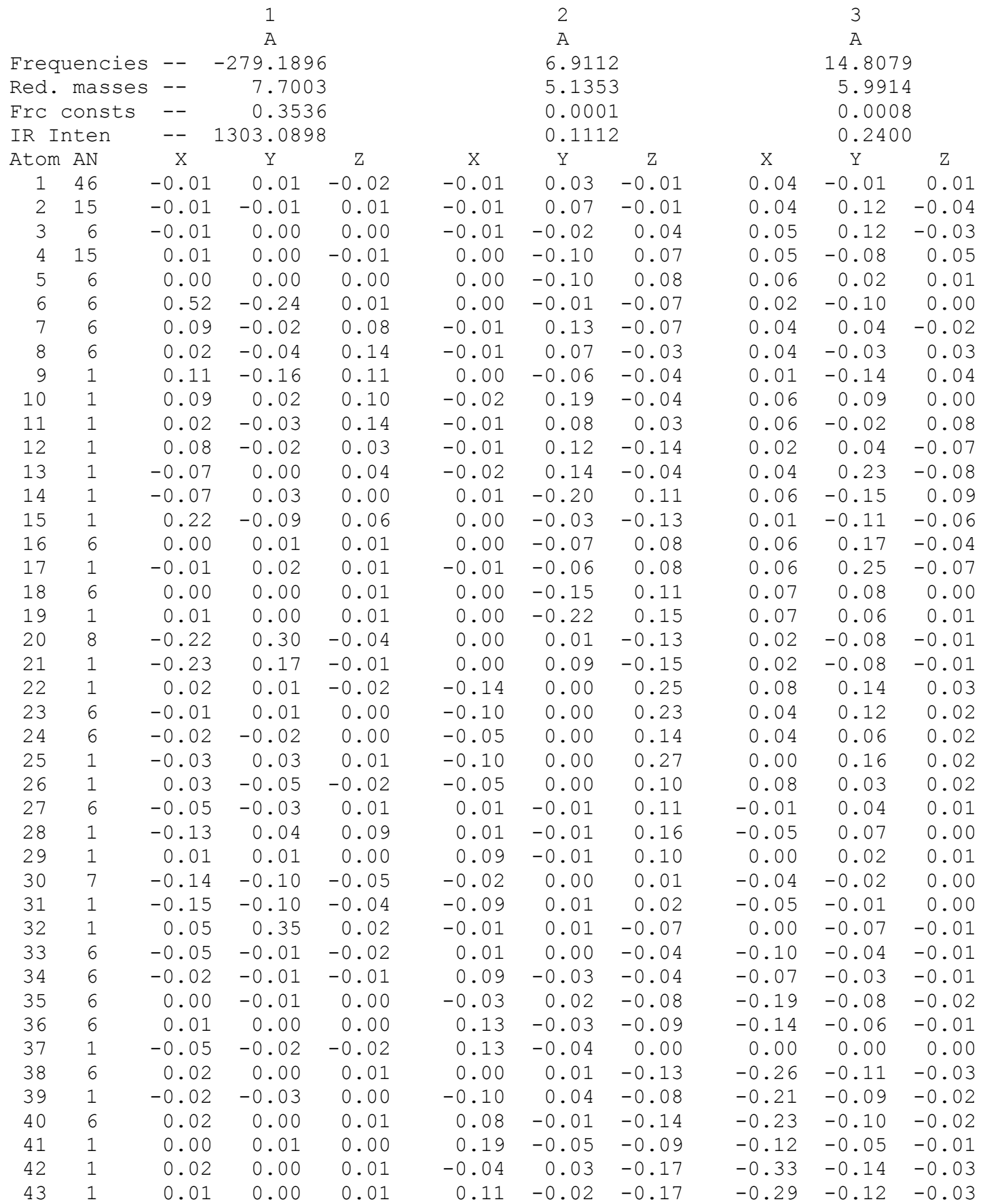


thermodynamics:

Sum of electronic and zero-point Energies=

Sum of electronic and thermal Enthalpies=

Total free energy in solution:

with all non electrostatic terms

ONIOM optimized geometry and PCM energy for 8ar.

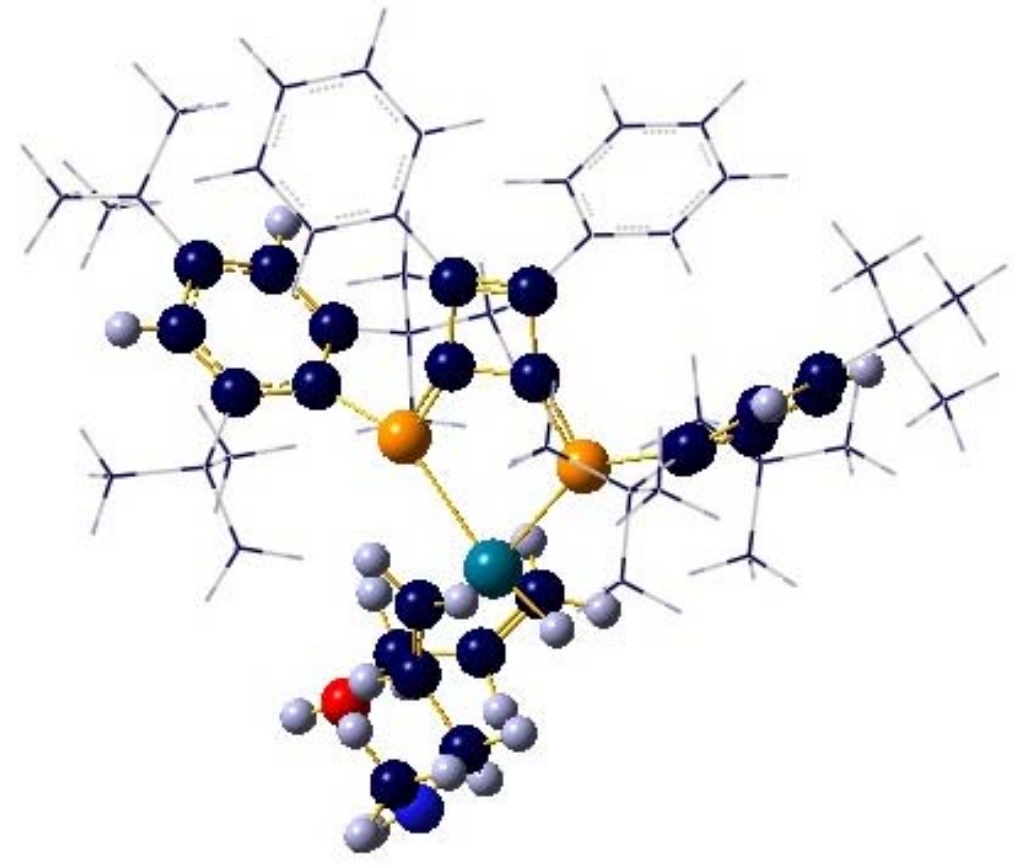

Standard orientation:

\begin{tabular}{|c|c|c|c|c|c|}
\hline \multirow{2}{*}{$\begin{array}{l}\text { Center } \\
\text { Number }\end{array}$} & \multirow{2}{*}{$\begin{array}{l}\text { Atomic } \\
\text { Number }\end{array}$} & \multirow{2}{*}{$\begin{array}{l}\text { Atomic } \\
\text { Type }\end{array}$} & \multicolumn{3}{|c|}{ Coordinates (Angstroms) } \\
\hline & & & $\mathrm{X}$ & Y & Z \\
\hline 1 & 6 & 10061003 & -2.142644 & 4.397229 & 2.046561 \\
\hline 2 & 6 & 10061002 & -0.012254 & 3.128184 & 2.680473 \\
\hline 3 & 6 & 10061002 & -0.657922 & 4.173506 & 2.044331 \\
\hline 4 & 1 & 10011000 & -2.698177 & 3.452683 & 2.021814 \\
\hline 5 & 1 & 10011000 & 1.013819 & 3.257271 & 3.007335 \\
\hline 6 & 1 & 10011000 & -0.083030 & 5.073404 & 1.828561 \\
\hline 7 & 1 & 10011000 & -2.385885 & 4.903781 & 2.995377 \\
\hline 8 & 1 & 10011000 & -0.554369 & 2.320208 & 3.160656 \\
\hline 9 & 8 & 10081003 & -2.491402 & 5.224770 & 0.947858 \\
\hline 10 & 1 & 10011000 & -3.382998 & 5.574902 & 1.085637 \\
\hline 11 & 7 & 10071003 & 0.517619 & 6.565252 & -2.161840 \\
\hline 12 & 1 & 10011000 & -0.364149 & 7.048706 & -2.019136 \\
\hline 13 & 6 & 10061003 & 0.751945 & 5.564675 & -1.134836 \\
\hline 14 & 1 & 10011000 & 1.768701 & 5.170433 & -1.268194 \\
\hline 15 & 1 & 10011000 & 0.717745 & 6.070299 & -0.163798 \\
\hline 16 & 6 & 10061002 & -0.261966 & 4.440035 & -1.193657 \\
\hline 17 & 6 & 10061002 & -0.030945 & 3.260786 & -1.847372 \\
\hline 18 & 1 & 10011000 & -1.276876 & 4.708283 & -0.901744 \\
\hline 19 & 1 & 10011000 & -0.840167 & 2.610411 & -2.154428 \\
\hline 20 & 1 & 10011000 & 0.933635 & 3.069118 & -2.304705 \\
\hline 21 & 6 & 10061003 & 0.682600 & 6.133310 & -3.539147 \\
\hline 22 & 1 & 10011000 & 1.715773 & 5.796315 & -3.687664 \\
\hline 23 & 1 & 10011000 & 0.008659 & 5.319501 & -3.858775 \\
\hline
\end{tabular}




\begin{tabular}{|c|c|c|c|c|c|}
\hline 24 & 1 & 10011000 & 0.519155 & 6.991067 & -4.198465 \\
\hline 25 & 15 & 10151003 & -1.661445 & 0.706898 & 0.291880 \\
\hline 26 & 46 & 10461004 & 0.134173 & 2.658184 & 0.481411 \\
\hline 27 & 6 & 10061000 & -0.730104 & -0.595172 & -0.213991 \\
\hline 28 & 15 & 10151003 & 1.690927 & 0.756560 & 0.203794 \\
\hline 29 & 1 & 10011000 & 1.383170 & 3.511085 & 0.548704 \\
\hline 30 & 6 & 10061000 & 0.786302 & -0.611606 & -0.120398 \\
\hline 31 & 6 & 10061000 & -0.645255 & -2.009629 & -0.605638 \\
\hline 32 & 6 & 10061000 & 0.714137 & -2.036448 & -0.462710 \\
\hline 33 & 6 & 10061000 & 3.488220 & 0.271406 & 0.203616 \\
\hline 34 & 6 & 10061000 & 4.097431 & -0.114458 & 1.452469 \\
\hline 35 & 6 & 10061000 & 4.195030 & 0.109861 & -1.040936 \\
\hline 36 & 6 & 10061000 & 5.237841 & -0.939048 & 1.364695 \\
\hline 37 & 6 & 10061000 & 5.366181 & -0.667834 & -0.995888 \\
\hline 38 & 6 & 10061000 & 5.873065 & -1.270157 & 0.161415 \\
\hline 39 & 1 & 10011000 & 5.651975 & -1.346222 & 2.274536 \\
\hline 40 & 1 & 10011000 & 5.936511 & -0.840360 & -1.898207 \\
\hline 41 & 6 & 10061000 & 1.726737 & -3.097966 & -0.588471 \\
\hline 42 & 6 & 10061000 & 1.682889 & -3.997282 & -1.664795 \\
\hline 43 & 6 & 10061000 & 2.713614 & -3.244238 & 0.394465 \\
\hline 44 & 6 & 10061000 & 2.615356 & -5.035477 & -1.750375 \\
\hline 45 & 1 & 10011000 & 0.930500 & -3.893783 & -2.436815 \\
\hline 46 & 6 & 10061000 & 3.634067 & -4.291868 & 0.315831 \\
\hline 47 & 1 & 10011000 & 2.746071 & -2.574762 & 1.240732 \\
\hline 48 & 6 & 10061000 & 3.587399 & -5.185991 & -0.757612 \\
\hline 49 & 1 & 10011000 & 2.580126 & -5.726997 & -2.582194 \\
\hline 50 & 1 & 10011000 & 4.382509 & -4.411409 & 1.088187 \\
\hline 51 & 1 & 10011000 & 4.304614 & -5.994024 & -0.820069 \\
\hline 52 & 6 & 10061000 & -1.638809 & -2.999970 & -1.058796 \\
\hline 53 & 6 & 10061000 & -2.720284 & -2.592582 & -1.850988 \\
\hline 54 & 6 & 10061000 & -1.484685 & -4.360296 & -0.748440 \\
\hline 55 & 6 & 10061000 & -3.622293 & -3.535223 & -2.351116 \\
\hline 56 & 1 & 10011000 & -2.842786 & -1.552971 & -2.113740 \\
\hline 57 & 6 & 10061000 & -2.391586 & -5.301507 & -1.244636 \\
\hline 58 & 1 & 10011000 & -0.666697 & -4.693241 & -0.122397 \\
\hline 59 & 6 & 10061000 & -3.453653 & -4.890613 & -2.054360 \\
\hline 60 & 1 & 10011000 & -4.448407 & -3.215651 & -2.972922 \\
\hline 61 & 1 & 10011000 & -2.267951 & -6.349764 & -1.005568 \\
\hline 62 & 1 & 10011000 & -4.152481 & -5.620322 & -2.442212 \\
\hline 63 & 6 & 10061000 & -3.419360 & 0.073085 & 0.441313 \\
\hline 64 & 6 & 10061000 & -4.373185 & 0.308172 & -0.611265 \\
\hline 65 & 6 & 10061000 & -3.744353 & -0.830211 & 1.526029 \\
\hline 66 & 6 & 10061000 & -5.440449 & -0.606243 & -0.721740 \\
\hline 67 & 6 & 10061000 & -4.865393 & -1.656017 & 1.330508 \\
\hline 68 & 6 & 10061000 & -5.687637 & -1.630090 & 0.197624 \\
\hline 69 & 1 & 10011000 & -6.096000 & -0.526560 & -1.575165 \\
\hline 70 & 1 & 10011000 & -5.139360 & -2.381634 & 2.084131 \\
\hline 71 & 6 & 10061003 & 3.801994 & 0.852344 & -2.361848 \\
\hline 72 & 6 & 10061003 & 2.468985 & 0.310229 & -2.935850 \\
\hline 73 & 6 & 10061003 & 3.706127 & 2.371724 & -2.089818 \\
\hline 74 & 6 & 10061003 & 4.855639 & 0.695351 & -3.494661 \\
\hline 75 & 1 & 10011000 & 2.424880 & -0.795959 & -2.837957 \\
\hline 76 & 1 & 10011000 & 1.581123 & 0.753100 & -2.454157 \\
\hline 77 & 1 & 10011000 & 2.359293 & 0.560715 & -4.013413 \\
\hline 78 & 1 & 10011000 & 4.694612 & 2.767995 & -1.771157 \\
\hline 79 & 1 & 10011000 & 3.390137 & 2.918076 & -3.005103 \\
\hline 80 & 1 & 10011000 & 2.986781 & 2.618438 & -1.290038 \\
\hline 81 & 1 & 10011000 & 4.576736 & 1.300925 & -4.385030 \\
\hline 82 & 1 & 10011000 & 5.856490 & 1.050499 & -3.165149 \\
\hline 83 & 1 & 10011000 & 4.924742 & -0.361450 & -3.833398 \\
\hline 84 & 6 & 10061003 & 7.166829 & -2.118671 & 0.083852 \\
\hline 85 & 6 & 10061003 & 7.532168 & -2.794789 & 1.428337 \\
\hline 86 & 6 & 10061003 & 7.014673 & -3.243239 & -0.967112 \\
\hline 87 & 6 & 10061003 & 8.345036 & -1.207059 & -0.320650 \\
\hline 88 & 1 & 10011000 & 7.786880 & -2.038762 & 2.202184 \\
\hline 89 & 1 & 10011000 & 6.693476 & -3.427175 & 1.791432 \\
\hline 90 & 1 & 10011000 & 8.423505 & -3.449270 & 1.312807 \\
\hline 91 & 1 & 10011000 & 6.873500 & -2.844916 & -1.992213 \\
\hline
\end{tabular}




\begin{tabular}{|c|c|c|c|c|c|}
\hline 92 & 1 & 10011000 & 7.923406 & -3.882923 & -0.993978 \\
\hline 93 & 1 & 10011000 & 6.150155 & -3.889439 & -0.721487 \\
\hline 94 & 1 & 10011000 & 9.297462 & -1.780054 & -0.34248 \\
\hline 95 & 1 & 10011000 & 8.193702 & -0.769597 & -1.32985 \\
\hline 96 & 1 & 10011000 & 8.455669 & -0.373330 & 0.40615 \\
\hline 97 & 6 & 10061003 & 3.655327 & 0.461171 & 2.8425 \\
\hline 98 & 6 & 10061003 & 3.660767 & 2.005067 & 2.7739 \\
\hline 99 & 6 & 10061003 & 2.255607 & -0.053927 & 3.2667 \\
\hline 100 & 6 & 10061003 & 4.626243 & 0.088832 & 3.9987 \\
\hline 101 & 1 & 10011000 & 4.683228 & 2.377180 & $2.5460^{\circ}$ \\
\hline 102 & 1 & 10011000 & 2.988058 & 2.392717 & 1.9891 \\
\hline 103 & 1 & 10011000 & 3.335723 & 2.445741 & 3.7414 \\
\hline 104 & 1 & 10011000 & 2.130433 & -1.123115 & 2.9989 \\
\hline 105 & 1 & 10011000 & 2.105475 & 0.036689 & 4.3645 \\
\hline 106 & 1 & 10011000 & 1.430968 & 0.533603 & 2.8262 \\
\hline 107 & 1 & 10011000 & 4.336520 & 0.599625 & 4.9433 \\
\hline 108 & 1 & 10011000 & 4.601952 & -1.003285 & 4.2053 \\
\hline 109 & 1 & 10011000 & 5.666972 & 0.404432 & 3.7671 \\
\hline 110 & 6 & 10061003 & -3.038471 & -0.808624 & 2.9273 \\
\hline 111 & 6 & 10061003 & -1.582038 & -1.328060 & 2.8475 \\
\hline 112 & 6 & 10061003 & -3.061968 & 0.628706 & 3.4851 \\
\hline 113 & 6 & 10061003 & -3.753935 & -1.683914 & 3.9955 \\
\hline 114 & 1 & 10011000 & -1.516436 & -2.199943 & 2.1627 \\
\hline 115 & 1 & 10011000 & -0.870044 & -0.544997 & 2.5304 \\
\hline 116 & 1 & 10011000 & -1.210672 & -1.654622 & 3.8432 \\
\hline 117 & 1 & 10011000 & -4.109117 & 0.978740 & 3.61439 \\
\hline 118 & 1 & 10011000 & -2.545152 & 0.684773 & 4.46794 \\
\hline 119 & 1 & 10011000 & -2.559352 & 1.331626 & 2.80621 \\
\hline 120 & 1 & 10011000 & -3.275688 & -1.567318 & 4.99300 \\
\hline 121 & 1 & 10011000 & -4.818579 & -1.385270 & 4.1128 \\
\hline 122 & 1 & 10011000 & -3.691905 & -2.762999 & 3.73496 \\
\hline 123 & 6 & 10061003 & -6.883920 & -2.606916 & 0.08922 \\
\hline 124 & 6 & 10061003 & -7.616871 & -2.518825 & -1.27204 \\
\hline 125 & 6 & 10061003 & -6.404194 & -4.067191 & 0.25713 \\
\hline 126 & 6 & 10061003 & -7.906165 & -2.280212 & 1.19841 \\
\hline 127 & 1 & 10011000 & -8.100749 & -1.527087 & -1.40426 \\
\hline 128 & 1 & 10011000 & -6.910732 & -2.700401 & -2.11097 \\
\hline 129 & 1 & 10011000 & -8.423330 & -3.281400 & -1.33745 \\
\hline 130 & 1 & 10011000 & -5.986516 & -4.259169 & 1.26623 \\
\hline 131 & 1 & 10011000 & -7.246976 & -4.778603 & 0.11788 \\
\hline 132 & 1 & 10011000 & -5.626552 & -4.305427 & -0.49346 \\
\hline 133 & 1 & 10011000 & -8.797090 & -2.940594 & 1.1198 \\
\hline 134 & 1 & 10011000 & -7.469611 & -2.423614 & 2.20922 \\
\hline 135 & 1 & 10011000 & -8.244912 & -1.224958 & 1.11291 \\
\hline 136 & 6 & 10061003 & -4.313540 & 1.529222 & -1.58657 \\
\hline 137 & 6 & 10061003 & -3.897459 & 2.821380 & -0.83727 \\
\hline 138 & 6 & 10061003 & -3.333871 & 1.230430 & -2.7383 \\
\hline 139 & 6 & 10061003 & -5.692592 & 1.856208 & -2.23242 \\
\hline 140 & 1 & 10011000 & -4.459848 & 2.915700 & 0.11658 \\
\hline 141 & 1 & 10011000 & -2.818988 & 2.864381 & -0.62583 \\
\hline 142 & 1 & 10011000 & -4.096601 & 3.731144 & -1.44381 \\
\hline 143 & 1 & 10011000 & -3.723803 & 0.406169 & -3.3736 \\
\hline 144 & 1 & 10011000 & -3.196698 & 2.127810 & -3.3798 \\
\hline 145 & 1 & 10011000 & -2.343124 & 0.911459 & -2.36966 \\
\hline 146 & 1 & 10011000 & -5.659251 & 2.814796 & -2.79470 \\
\hline 147 & 1 & 10011000 & -5.996589 & 1.094101 & -2.98085 \\
\hline 148 & 1 & 10011000 & -6.479918 & 1.944889 & -1.4525 \\
\hline
\end{tabular}

Total free energy in solution:

with all non electrostatic terms

$\left(\mathrm{a} \cdot \mathrm{u}_{\text {. }}\right)=-3236.949533$

ONIOM optimized geometry and PCM energy for 12ar. 


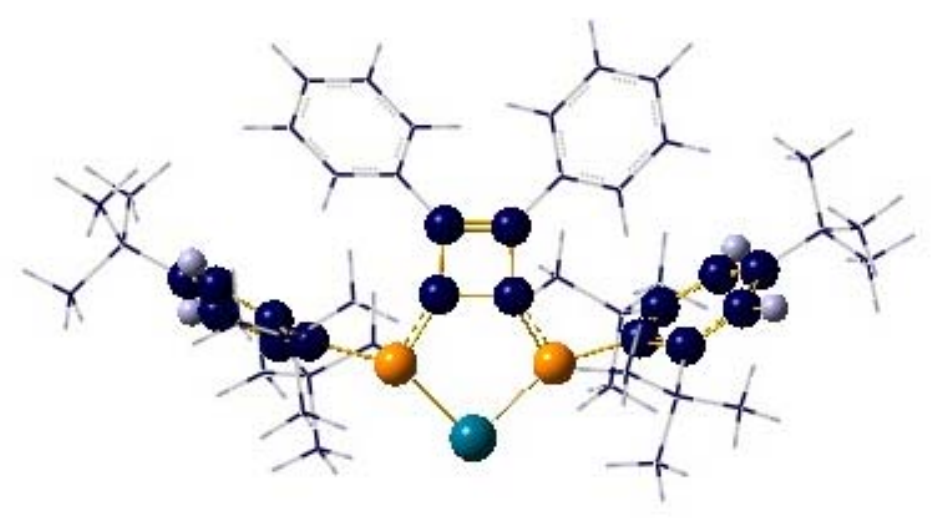

Standard orientation:

\begin{tabular}{|c|c|c|c|c|c|}
\hline \multirow{2}{*}{$\begin{array}{l}\text { Center } \\
\text { Number }\end{array}$} & \multirow{2}{*}{$\begin{array}{l}\text { Atomic } \\
\text { Number }\end{array}$} & \multirow{2}{*}{$\begin{array}{l}\text { Atomic } \\
\text { Type }\end{array}$} & \multicolumn{3}{|c|}{ Coordinates (Angstroms) } \\
\hline & & & $\mathrm{X}$ & $\mathrm{Y}$ & Z \\
\hline 1 & 46 & 10461004 & -.072913 & -2.947590 & .526918 \\
\hline 2 & 15 & 10151003 & 1.620369 & -1.439801 & .256513 \\
\hline 3 & 6 & 10061002 & .779239 & .022436 & .272051 \\
\hline 4 & 15 & 10151003 & -1.676267 & -1.315881 & .611539 \\
\hline 5 & 6 & 10061002 & -.768431 & .071777 & .283665 \\
\hline 6 & 6 & 10061000 & -.638094 & 1.507806 & .068809 \\
\hline 7 & 6 & 10061000 & .739834 & 1.464794 & .104952 \\
\hline 8 & 6 & 10061002 & -3.468071 & -.886351 & .285882 \\
\hline 9 & 6 & 10061000 & -4.242058 & -.166855 & 1.265685 \\
\hline 10 & 6 & 10061000 & -4.045168 & -1.225158 & -.992751 \\
\hline 11 & 6 & 10061000 & -5.415689 & .467057 & .805672 \\
\hline 12 & 6 & 10061000 & -5.289875 & -.640306 & -1.293663 \\
\hline 13 & 6 & 10061000 & -5.953505 & .277728 & -.472159 \\
\hline 14 & 1 & 10011000 & -5.935565 & 1.142986 & 1.469308 \\
\hline 15 & 1 & 10011000 & -5.796276 & -.871607 & -2.221341 \\
\hline 16 & 6 & 10061002 & 3.417303 & -.996766 & -.030178 \\
\hline 17 & 6 & 10061000 & 4.314092 & -.882047 & 1.092076 \\
\hline 18 & 6 & 10061000 & 3.880123 & -.690444 & -1.361991 \\
\hline 19 & 6 & 10061000 & 5.499992 & -.146435 & .884004 \\
\hline 20 & 6 & 10061000 & 5.113979 & -.019347 & -1.457379 \\
\hline 21 & 6 & 10061000 & 5.909405 & .339657 & -.362939 \\
\hline 22 & 1 & 10011000 & 6.129517 & .073356 & 1.733531 \\
\hline 23 & 1 & 10011000 & 5.504262 & .270398 & -2.424358 \\
\hline 24 & 6 & 10061000 & 1.790335 & 2.490569 & -.022310 \\
\hline 25 & 6 & 10061000 & 1.617816 & 3.583719 & -.885463 \\
\hline 26 & 6 & 10061000 & 2.954246 & 2.399128 & .751454 \\
\hline 27 & 6 & 10061000 & 2.604013 & 4.571108 & -.973279 \\
\hline 28 & 1 & 10011000 & .725446 & 3.668964 & -1.493066 \\
\hline 29 & 6 & 10061000 & 3.932331 & 3.392981 & .672195 \\
\hline 30 & 1 & 10011000 & 3.086616 & 1.580936 & 1.442864 \\
\hline 31 & 6 & 10061000 & 3.759890 & 4.477018 & -.193047 \\
\hline 32 & 1 & 10011000 & 2.470003 & 5.411247 & -1.642224 \\
\hline 33 & 1 & 10011000 & 4.822667 & 3.323449 & 1.283675 \\
\hline 34 & 1 & 10011000 & 4.520514 & 5.244257 & -.257156 \\
\hline 35 & 6 & 10061000 & -1.613314 & 2.614217 & .104845 \\
\hline 36 & 6 & 10061000 & -2.867505 & 2.472204 & -.504035 \\
\hline 37 & 6 & 10061000 & -1.305131 & 3.806913 & .780203 \\
\hline 38 & 6 & 10061000 & -3.805242 & 3.506709 & -.429092 \\
\hline 39 & 1 & 10011000 & -3.113363 & 1.571317 & -1.049152 \\
\hline 40 & 6 & 10061000 & -2.244785 & 4.839073 & .851177 \\
\hline 41 & 1 & 10011000 & -.349885 & 3.930414 & 1.274195 \\
\hline 42 & 6 & 10061000 & -3.495779 & 4.687879 & .249066 \\
\hline 43 & 1 & 10011000 & -4.771218 & 3.396823 & -.897437 \\
\hline 44 & 1 & 10011000 & -2.005670 & 5.752924 & 1.379446 \\
\hline 45 & 1 & 10011000 & -4.224660 & 5.485871 & .306560 \\
\hline 46 & 6 & 10061003 & 3.145195 & -1.154282 & -2.670679 \\
\hline 47 & 6 & 10061003 & 2.900009 & -2.682582 & -2.630854 \\
\hline
\end{tabular}




\begin{tabular}{|c|c|c|c|c|c|}
\hline 48 & 6 & 10061003 & 1.807652 & -.398763 & -2.864699 \\
\hline 49 & 6 & 10061003 & 3.977556 & -.900005 & -3.960267 \\
\hline 50 & 1 & 10011000 & 3.857228 & -3.220331 & -2.457535 \\
\hline 51 & 1 & 10011000 & 2.189417 & -2.988424 & -1.843694 \\
\hline 52 & 1 & 10011000 & 2.468647 & -3.039864 & -3.591263 \\
\hline 53 & 1 & 10011000 & 1.918991 & .669934 & -2.582111 \\
\hline 54 & 1 & 10011000 & 1.468793 & -.436288 & -3.922883 \\
\hline 55 & 1 & 10011000 & .979289 & -.847583 & -2.290182 \\
\hline 56 & 1 & 10011000 & 3.464917 & -1.311828 & -4.857339 \\
\hline 57 & 1 & 10011000 & 4.109123 & .188543 & -4.144678 \\
\hline 58 & 1 & 10011000 & 4.970785 & -1.396257 & -3.899700 \\
\hline 59 & 6 & 10061003 & 7.231659 & 1.114146 & -.589944 \\
\hline 60 & 6 & 10061003 & 6.962603 & 2.420515 & -1.372674 \\
\hline 61 & 6 & 10061003 & 7.939051 & 1.508587 & .729763 \\
\hline 62 & 6 & 10061003 & 8.204493 & .230407 & -1.399723 \\
\hline 63 & 1 & 10011000 & 6.556799 & 2.226788 & -2.386294 \\
\hline 64 & 1 & 10011000 & 6.243026 & 3.059840 & -.824661 \\
\hline 65 & 1 & 10011000 & 7.902047 & 2.999522 & -1.506813 \\
\hline 66 & 1 & 10011000 & 8.271206 & .608762 & 1.291275 \\
\hline 67 & 1 & 10011000 & 8.847966 & 2.115392 & .525284 \\
\hline 68 & 1 & 10011000 & 7.264702 & 2.116704 & 1.370981 \\
\hline 69 & 1 & 10011000 & 9.181200 & .743220 & -1.537603 \\
\hline 70 & 1 & 10011000 & 8.386047 & -.729756 & -.869884 \\
\hline 71 & 1 & 10011000 & 7.802764 & -.000399 & -2.408592 \\
\hline 72 & 6 & 10061003 & 4.076153 & -1.590718 & 2.473434 \\
\hline 73 & 6 & 10061003 & 2.999588 & -.850220 & 3.298230 \\
\hline 74 & 6 & 10061003 & 3.672045 & -3.074369 & 2.265811 \\
\hline 75 & 6 & 10061003 & 5.355262 & -1.629931 & 3.360261 \\
\hline 76 & 1 & 10011000 & 3.282361 & .215545 & 3.431676 \\
\hline 77 & 1 & 10011000 & 1.997195 & -.886416 & 2.839376 \\
\hline 78 & 1 & 10011000 & 2.899854 & -1.306903 & 4.306963 \\
\hline 79 & 1 & 10011000 & 4.334695 & -3.554046 & 1.513443 \\
\hline 80 & 1 & 10011000 & 3.751335 & -3.653916 & 3.211025 \\
\hline 81 & 1 & 10011000 & 2.622722 & -3.198866 & 1.948495 \\
\hline 82 & 1 & 10011000 & 5.196247 & -2.244441 & 4.273334 \\
\hline 83 & 1 & 10011000 & 6.209503 & -2.072780 & 2.803158 \\
\hline 84 & 1 & 10011000 & 5.630875 & -.618024 & 3.727754 \\
\hline 85 & 6 & 10061003 & -3.391464 & -2.227400 & -2.012038 \\
\hline 86 & 6 & 10061003 & -2.022902 & -1.699159 & -2.524246 \\
\hline 87 & 6 & 10061003 & -3.224831 & -3.615995 & -1.349067 \\
\hline 88 & 6 & 10061003 & -4.262560 & -2.471772 & -3.277690 \\
\hline 89 & 1 & 10011000 & -2.015961 & -.588276 & -2.557654 \\
\hline 90 & 1 & 10011000 & -1.175492 & -2.053305 & -1.906830 \\
\hline 91 & 1 & 10011000 & -1.789351 & -2.063996 & -3.548030 \\
\hline 92 & 1 & 10011000 & -4.209091 & -3.992788 & -.995695 \\
\hline 93 & 1 & 10011000 & -2.809567 & -4.350476 & -2.073102 \\
\hline 94 & 1 & 10011000 & -2.540696 & -3.604805 & -.482784 \\
\hline 95 & 1 & 10011000 & -3.781970 & -3.208967 & -3.958027 \\
\hline 96 & 1 & 10011000 & -5.256009 & -2.892581 & -3.008802 \\
\hline 97 & 1 & 10011000 & -4.393515 & -1.532452 & -3.858193 \\
\hline 98 & 6 & 10061003 & -3.909281 & -.159714 & 2.800052 \\
\hline 99 & 6 & 10061003 & -3.710716 & -1.608712 & 3.309283 \\
\hline 100 & 6 & 10061003 & -2.654574 & .691189 & 3.102916 \\
\hline 101 & 6 & 10061003 & -5.057086 & .432466 & 3.668176 \\
\hline 102 & 1 & 10011000 & -4.590749 & -2.233290 & 3.042895 \\
\hline 103 & 1 & 10011000 & -2.808133 & -2.092967 & 2.898825 \\
\hline 104 & 1 & 10011000 & -3.591302 & -1.629763 & 4.414406 \\
\hline 105 & 1 & 10011000 & -2.705863 & 1.659432 & 2.562603 \\
\hline 106 & 1 & 10011000 & -2.576525 & .912456 & 4.189663 \\
\hline 107 & 1 & 10011000 & -1.713118 & .176705 & 2.845265 \\
\hline 108 & 1 & 10011000 & -4.842245 & .316527 & 4.753243 \\
\hline 109 & 1 & 10011000 & -5.175446 & 1.523708 & 3.491990 \\
\hline 110 & 1 & 10011000 & -6.018700 & -.088094 & 3.466595 \\
\hline 111 & 6 & 10061003 & -7.273922 & .929054 & -.952239 \\
\hline 112 & 6 & 10061003 & -7.791181 & 2.028831 & .007643 \\
\hline 113 & 6 & 10061003 & -7.077064 & 1.584829 & -2.340774 \\
\hline 114 & 6 & 10061003 & -8.364281 & -.157883 & -1.056767 \\
\hline 115 & 1 & 10011000 & -8.079149 & 1.600282 & .991782 \\
\hline
\end{tabular}




\begin{tabular}{rrrrrr}
116 & 1 & 10011000 & -7.019849 & 2.814355 & .158956 \\
117 & 1 & 10011000 & -8.698581 & 2.520543 & -.405681 \\
118 & 1 & 10011000 & -6.881137 & .833838 & -3.133147 \\
119 & 1 & 10011000 & -7.988940 & 2.140969 & -2.648846 \\
120 & 1 & 10011000 & -6.227674 & 2.297093 & -2.321610 \\
121 & 1 & 10011000 & -9.334392 & .286528 & -1.368720 \\
122 & 1 & 10011000 & -8.094239 & -.934589 & -1.802844 \\
123 & 1 & 10011000 & -8.508090 & -.656677 & -.073824 \\
\hline
\end{tabular}

Total free energy in solution:

with all non electrostatic terms (a.u.) $=-2830.999724$

\section{DAD-H}

Optimized geometry, three lower frequencies, thermochemistry and PCM energy for $\mathbf{1 b}$.

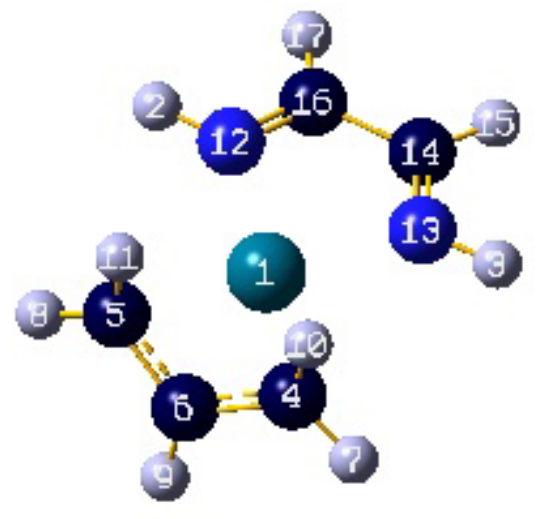

\begin{tabular}{cccrrr}
$\begin{array}{c}\text { Center } \\
\text { Number }\end{array}$ & $\begin{array}{c}\text { Atomic } \\
\text { Number }\end{array}$ & $\begin{array}{c}\text { Atomic } \\
\text { Type }\end{array}$ & \multicolumn{2}{c}{ Coordinates (Angstroms) } \\
-1 & 46 & 0 & -0.253934 & -0.000019 & -0.021445 \\
1 & 1 & 0 & 1.440465 & -2.325650 & 0.013533 \\
2 & 1 & 0 & 1.440364 & 2.325667 & 0.013525 \\
3 & 6 & 0 & -2.007382 & 1.204441 & -0.186710 \\
4 & 6 & 0 & -2.007477 & -1.204397 & -0.186726 \\
5 & 6 & 0 & -2.355279 & 0.000030 & 0.460095 \\
6 & 1 & 0 & -2.051702 & 2.141371 & 0.363141 \\
7 & 1 & 0 & -2.051878 & -2.141337 & 0.363099 \\
8 & 1 & 0 & -2.585572 & 0.000029 & 1.524401 \\
9 & 1 & 0 & -2.084201 & 1.287119 & -1.272126 \\
10 & 1 & 0 & -2.084272 & -1.287038 & -1.272145 \\
11 & 7 & 0 & 1.425554 & -1.305852 & 0.010301 \\
12 & 7 & 0 & 1.425501 & 1.305847 & 0.010276 \\
13 & 6 & 0 & 2.574173 & 0.740557 & 0.038325 \\
14 & 1 & 0 & 3.515421 & 1.291117 & 0.064585 \\
15 & 6 & 0 & 2.574211 & -0.740513 & 0.038317 \\
16 & 1 & 0 & 3.515468 & -1.291054 & 0.064606
\end{tabular}

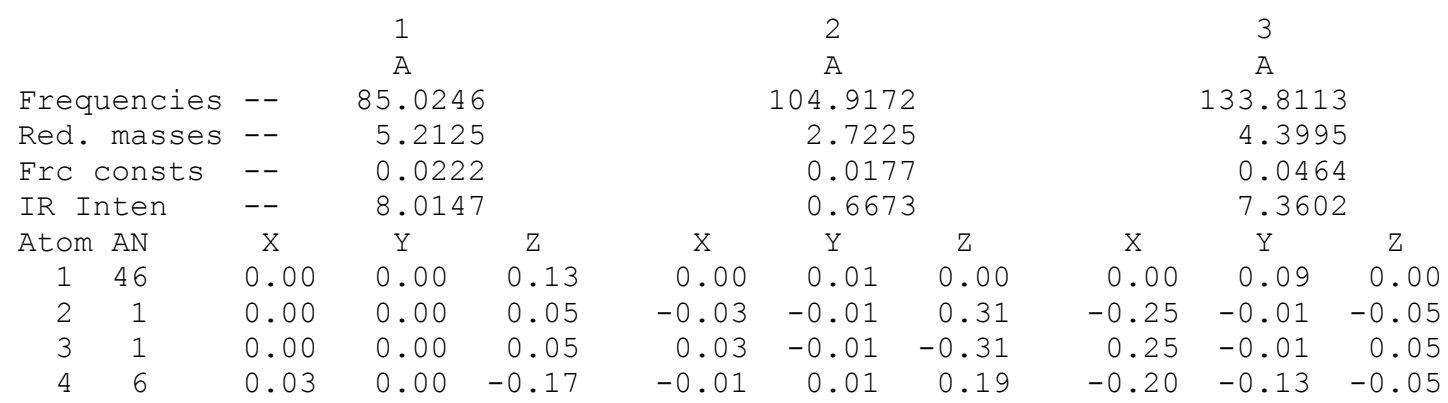




$\begin{array}{rrrrrrrrrrr}5 & 6 & 0.03 & 0.00 & -0.17 & 0.01 & 0.01 & -0.19 & 0.20 & -0.13 & 0.05 \\ 6 & 6 & -0.09 & 0.00 & -0.24 & 0.00 & -0.10 & 0.00 & 0.00 & -0.15 & 0.00 \\ 7 & 1 & -0.07 & 0.00 & -0.17 & -0.01 & -0.08 & 0.35 & -0.37 & -0.11 & -0.10 \\ 8 & 1 & -0.07 & 0.00 & -0.17 & 0.01 & -0.08 & -0.35 & 0.37 & -0.11 & 0.10 \\ 9 & 1 & -0.28 & 0.00 & -0.28 & 0.00 & -0.27 & 0.00 & 0.00 & -0.15 & 0.00 \\ 10 & 1 & 0.23 & 0.01 & -0.18 & -0.05 & 0.19 & 0.21 & -0.19 & -0.20 & -0.05 \\ 11 & 1 & 0.23 & -0.01 & -0.18 & 0.05 & 0.19 & -0.21 & 0.19 & -0.20 & 0.05 \\ 12 & 7 & 0.00 & 0.00 & 0.03 & -0.02 & -0.01 & 0.13 & -0.13 & -0.01 & -0.02 \\ 13 & 7 & 0.00 & 0.00 & 0.03 & 0.02 & -0.01 & -0.13 & 0.13 & -0.01 & 0.02 \\ 14 & 6 & 0.01 & 0.00 & -0.23 & 0.01 & -0.02 & -0.12 & 0.07 & -0.13 & 0.02 \\ 15 & 1 & 0.02 & 0.00 & -0.42 & 0.02 & -0.03 & -0.28 & 0.13 & -0.23 & 0.05 \\ 16 & 6 & 0.01 & 0.00 & -0.23 & -0.01 & -0.02 & 0.12 & -0.07 & -0.13 & -0.02 \\ 17 & 1 & 0.02 & 0.00 & -0.42 & -0.02 & -0.03 & 0.28 & -0.13 & -0.23 & -0.05\end{array}$

thermodynamics:

Sum of electronic and zero-point Energies=

$-431.766701$

Sum of electronic and thermal Enthalpies=

$-431.756881$

Sum of electronic and thermal Free Energies=

$-431.800955$

Total free energy in solution:

with all non electrostatic terms

$-431.975869$

Optimized geometry, three lower frequencies, thermochemistry and PCM energy for $\mathbf{T} \mathbf{S}_{\mathbf{1 b}-\mathbf{2 b}}$.

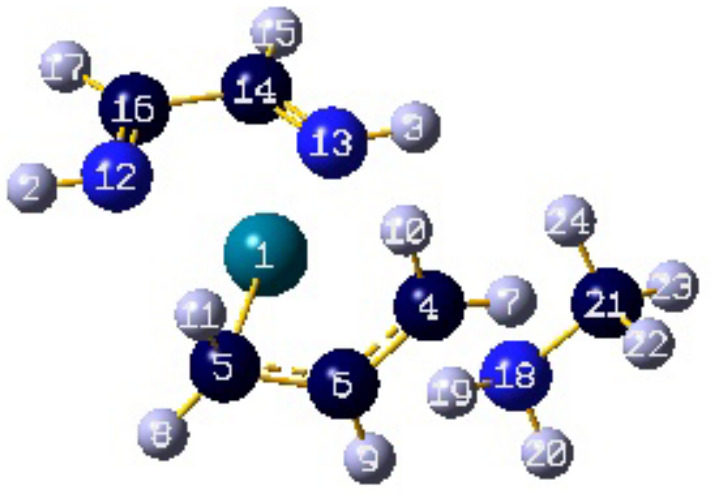

\begin{tabular}{|c|c|c|c|c|c|}
\hline \multirow{2}{*}{$\begin{array}{l}\text { Center } \\
\text { Number }\end{array}$} & \multirow{2}{*}{$\begin{array}{l}\text { Atomic } \\
\text { Number }\end{array}$} & \multirow{2}{*}{$\begin{array}{c}\text { Atomic } \\
\text { Type }\end{array}$} & \multicolumn{3}{|c|}{ Coordinates (Angstroms) } \\
\hline & & & $\mathrm{X}$ & $\mathrm{Y}$ & Z \\
\hline 1 & 46 & 0 & -0.606744 & -0.249968 & -0.092384 \\
\hline 2 & 1 & 0 & -2.942408 & -1.606442 & 0.948281 \\
\hline 3 & 1 & 0 & -1.483970 & 2.485526 & -0.737900 \\
\hline 4 & 6 & 0 & 1.912553 & 0.294534 & -0.134744 \\
\hline 5 & 6 & 0 & 0.685289 & -1.852247 & 0.060181 \\
\hline 6 & 6 & 0 & 1.338461 & -0.854358 & -0.725470 \\
\hline 7 & 1 & 0 & 2.052404 & 1.192053 & -0.728582 \\
\hline 8 & 1 & 0 & 0.359763 & -2.771132 & -0.424261 \\
\hline 9 & 1 & 0 & 1.390378 & -0.960639 & -1.808144 \\
\hline 10 & 1 & 0 & 1.865591 & 0.437780 & 0.940909 \\
\hline 11 & 1 & 0 & 0.945786 & -1.967842 & 1.114392 \\
\hline 12 & 7 & 0 & -2.594654 & -0.720395 & 0.582966 \\
\hline 13 & 7 & 0 & -1.767827 & 1.576871 & -0.372448 \\
\hline 14 & 6 & 0 & -3.007812 & 1.500008 & -0.059277 \\
\hline 15 & 1 & 0 & -3.712088 & 2.326581 & -0.168078 \\
\hline 16 & 6 & 0 & -3.475957 & 0.202307 & 0.472521 \\
\hline 17 & 1 & 0 & -4.524504 & 0.071771 & 0.745532 \\
\hline 18 & 7 & 0 & 4.004960 & 0.044162 & -0.021018 \\
\hline 19 & 1 & 0 & 4.129046 & -0.827975 & 0.490205 \\
\hline 20 & 1 & 0 & 4.309972 & -0.126079 & -0.977911 \\
\hline
\end{tabular}




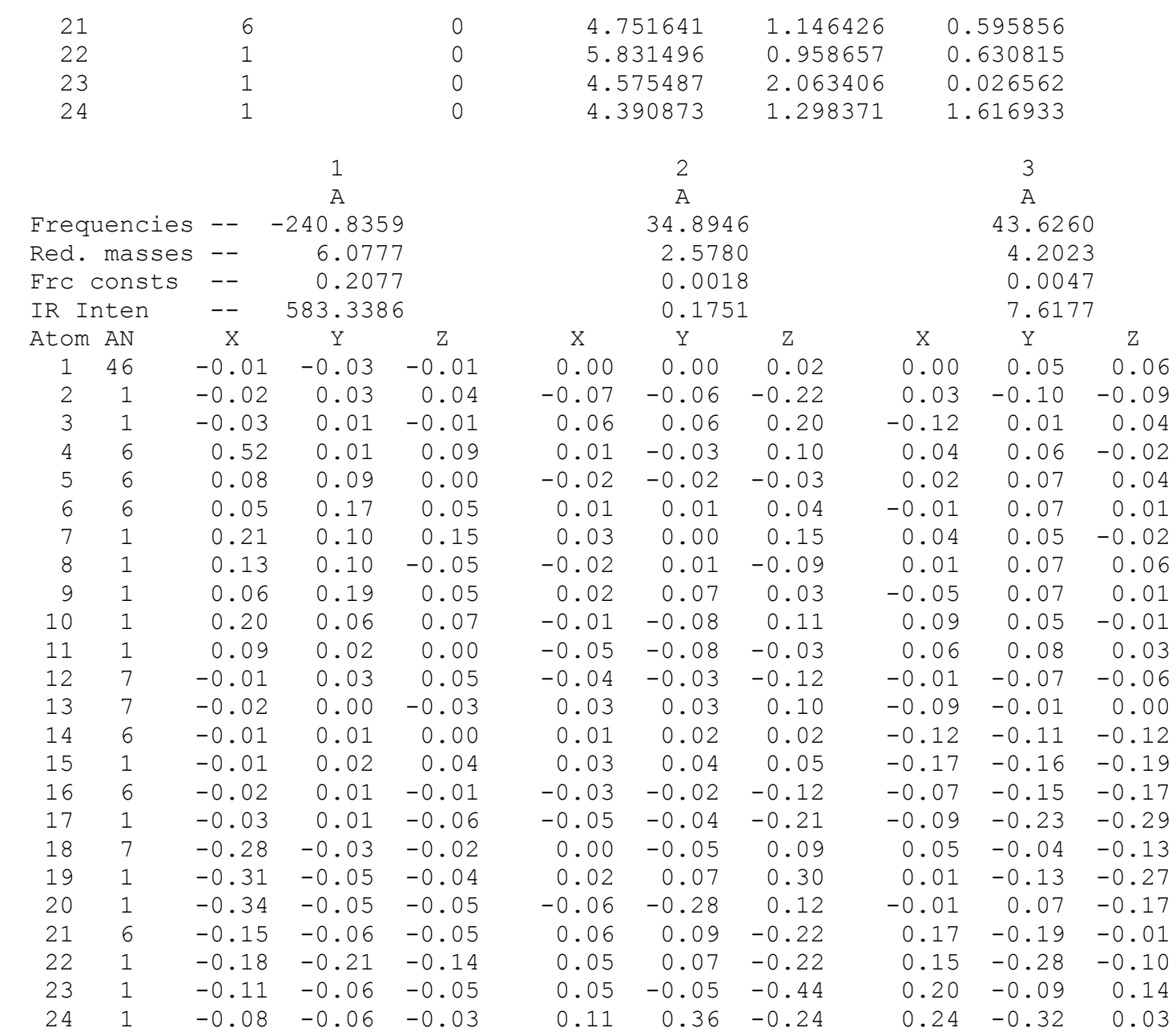

thermodynamics:

Sum of electronic and zero-point Energies=

$-527.519771$

Sum of electronic and thermal Enthalpies=

$-527.505769$

Sum of electronic and thermal Free Energies=

$-527.561205$

Total free energy in solution:

with all non electrostatic terms

$(\mathrm{a} \cdot \mathrm{u} \cdot)=-527.784307$

Optimized geometry, three lower frequencies, thermochemistry and PCM energy for $\mathbf{2 b}$.

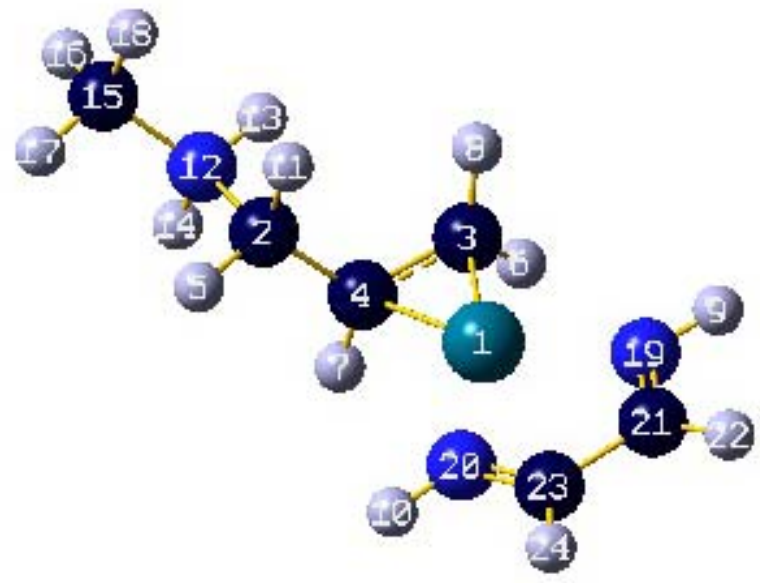




\begin{tabular}{rrrrrr}
$\begin{array}{c}\text { Center } \\
\text { Number }\end{array}$ & $\begin{array}{c}\text { Atomic } \\
\text { Number }\end{array}$ & $\begin{array}{c}\text { Atomic } \\
\text { Type }\end{array}$ & \multicolumn{1}{c}{ Coordinates (Angstroms) } \\
-1 & 46 & 0 & -0.621063 & -0.239898 & -0.133169 \\
1 & 6 & 0 & 2.295057 & 0.531482 & 0.073852 \\
2 & 6 & 0 & 0.922300 & -1.592759 & -0.160060 \\
3 & 6 & 0 & 1.373730 & -0.342783 & -0.705112 \\
4 & 1 & 0 & 2.310745 & 1.572931 & -0.260718 \\
5 & 1 & 0 & 0.732148 & -2.434570 & -0.825800 \\
6 & 1 & 0 & 1.465780 & -0.244925 & -1.790504 \\
7 & 1 & 0 & 1.221099 & -1.890564 & 0.849562 \\
8 & 1 & 0 & -2.833456 & -1.769652 & 0.982448 \\
9 & 1 & 0 & -1.756005 & 2.429520 & -0.714757 \\
10 & 1 & 0 & 2.106090 & 0.499765 & 1.150328 \\
11 & 7 & 0 & 3.754855 & 0.034849 & -0.083784 \\
12 & 1 & 0 & 3.753005 & -0.955895 & 0.181938 \\
13 & 1 & 0 & 3.978663 & 0.042455 & -1.084042 \\
14 & 6 & 0 & 4.789960 & 0.784931 & 0.682749 \\
15 & 1 & 0 & 5.772411 & 0.344453 & 0.501181 \\
16 & 1 & 0 & 4.781146 & 1.826108 & 0.356248 \\
17 & 1 & 0 & 4.545835 & 0.728935 & 1.744748 \\
18 & 7 & 0 & -2.563190 & -0.862267 & 0.604390 \\
19 & 7 & 0 & -1.946290 & 1.502175 & -0.336135 \\
20 & 6 & 0 & -3.509809 & 0.001082 & 0.567115 \\
21 & 1 & 0 & -4.527956 & -0.198983 & 0.906336 \\
22 & 6 & 0 & -3.164874 & 1.328896 & 0.023669 \\
23 & 1 & 0 & -3.936399 & 2.097334 & -0.055794 \\
24 & & 0 & & &
\end{tabular}

\begin{tabular}{|c|c|c|c|c|c|c|c|c|c|c|}
\hline & & & 1 & & & 2 & & & 3 & \\
\hline & & & A & & & A & & & A & \\
\hline Frequ & encies & -- & 40.527 & & & 53.71 & & & 77.922 & \\
\hline Red. & masses & -- & 4.910 & & & 3.65 & & & 2.318 & \\
\hline Frc $c$ & onsts & -- & 0.004 & & & 0.00 & & & 0.008 & \\
\hline IR In & ten & -- & 10.768 & & & 4.57 & & & 10.830 & \\
\hline Atom & AN & X & $\mathrm{Y}$ & Z & $\mathrm{x}$ & $\mathrm{Y}$ & Z & $\mathrm{x}$ & Y & Z \\
\hline 1 & 46 & 0.01 & 0.05 & 0.09 & 0.00 & -0.05 & 0.01 & 0.01 & -0.02 & 0.04 \\
\hline 2 & 6 & 0.06 & 0.00 & 0.02 & -0.03 & -0.06 & 0.06 & -0.04 & -0.02 & -0.01 \\
\hline 3 & 6 & 0.00 & 0.04 & 0.01 & -0.02 & -0.08 & 0.02 & 0.01 & -0.04 & -0.10 \\
\hline 4 & 6 & -0.01 & 0.06 & 0.04 & 0.00 & -0.08 & 0.05 & -0.03 & 0.00 & -0.05 \\
\hline 5 & 1 & 0.08 & 0.01 & 0.06 & -0.11 & -0.05 & 0.09 & -0.14 & 0.02 & 0.11 \\
\hline 6 & 1 & -0.03 & 0.06 & 0.00 & -0.01 & -0.07 & 0.00 & -0.02 & 0.00 & -0.14 \\
\hline 7 & 1 & -0.05 & 0.09 & 0.04 & 0.00 & -0.06 & 0.05 & -0.06 & 0.06 & -0.04 \\
\hline 8 & 1 & 0.03 & 0.01 & 0.00 & -0.05 & -0.10 & 0.03 & 0.06 & -0.10 & -0.13 \\
\hline 9 & 1 & -0.04 & -0.09 & -0.19 & -0.18 & -0.01 & -0.28 & -0.04 & 0.08 & 0.12 \\
\hline 10 & 1 & -0.03 & 0.04 & 0.14 & 0.22 & 0.10 & 0.25 & 0.09 & -0.03 & -0.08 \\
\hline 11 & 1 & 0.13 & -0.03 & 0.03 & 0.00 & -0.10 & 0.06 & 0.01 & -0.15 & 0.00 \\
\hline 12 & 7 & 0.03 & -0.05 & -0.10 & 0.00 & 0.05 & 0.00 & -0.01 & 0.11 & -0.13 \\
\hline 13 & 1 & 0.02 & -0.06 & -0.12 & 0.11 & 0.08 & 0.08 & 0.04 & 0.04 & -0.39 \\
\hline 14 & 1 & -0.04 & -0.04 & -0.11 & -0.07 & 0.00 & -0.02 & 0.00 & 0.39 & -0.12 \\
\hline 15 & 6 & 0.12 & -0.11 & -0.16 & -0.02 & 0.21 & -0.13 & -0.08 & -0.02 & 0.09 \\
\hline 16 & 1 & 0.09 & -0.15 & -0.24 & 0.01 & 0.28 & -0.14 & -0.04 & 0.13 & -0.05 \\
\hline 17 & 1 & 0.15 & -0.10 & -0.15 & -0.12 & 0.18 & -0.23 & -0.18 & 0.08 & 0.41 \\
\hline 18 & 1 & 0.20 & -0.11 & -0.15 & 0.05 & 0.30 & -0.11 & -0.06 & -0.37 & 0.07 \\
\hline 19 & 7 & -0.04 & -0.06 & -0.12 & -0.09 & 0.01 & -0.14 & -0.01 & 0.05 & 0.06 \\
\hline 20 & 7 & -0.04 & 0.01 & 0.05 & 0.13 & 0.06 & 0.11 & 0.06 & 0.00 & -0.03 \\
\hline 21 & 6 & -0.10 & -0.13 & -0.27 & -0.03 & 0.08 & -0.12 & 0.00 & 0.06 & -0.04 \\
\hline 22 & 1 & -0.14 & -0.22 & -0.47 & -0.07 & 0.11 & -0.23 & -0.01 & 0.10 & -0.05 \\
\hline 23 & 6 & -0.09 & -0.09 & -0.16 & 0.10 & 0.12 & 0.07 & 0.03 & 0.02 & -0.11 \\
\hline 24 & 1 & -0.12 & -0.13 & -0.25 & 0.17 & 0.20 & 0.15 & 0.04 & 0.01 & -0.22 \\
\hline
\end{tabular}

thermodynamics:

Sum of electronic and zero-point Energies=

$-527.530454$

Sum of electronic and thermal Enthalpies=

$-527.516841$

Sum of electronic and thermal Free Energies=

$-527.570904$ 
Total free energy in solution:

with all non electrostatic terms (a.u.) = $\quad-527.811611$

Optimized geometry, three lower frequencies, thermochemistry and PCM energy for $\mathbf{1 1 b}$.

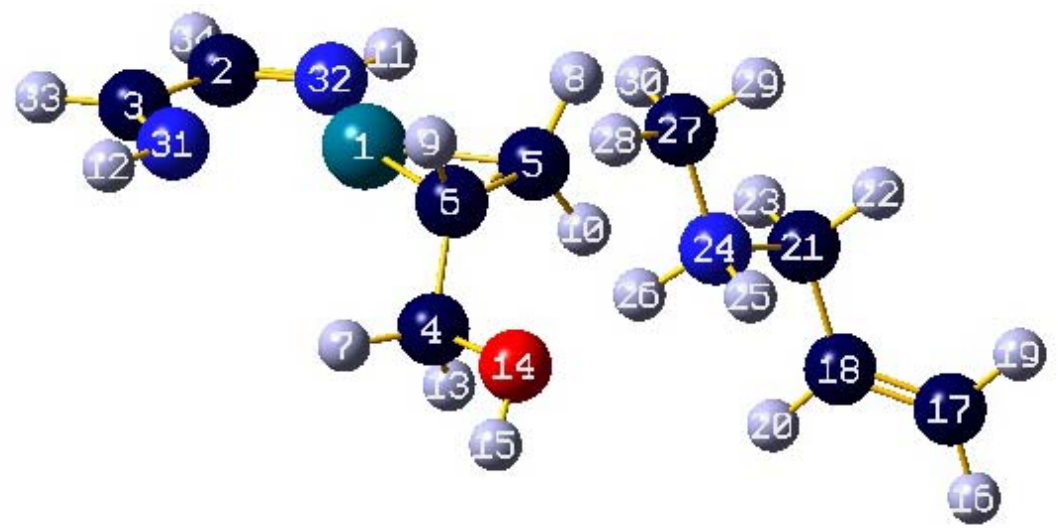

\begin{tabular}{|c|c|c|c|c|c|}
\hline \multirow{2}{*}{$\begin{array}{l}\text { Center } \\
\text { Number }\end{array}$} & \multirow{2}{*}{$\begin{array}{l}\text { Atomic } \\
\text { Number }\end{array}$} & \multirow{2}{*}{$\begin{array}{c}\text { Atomic } \\
\text { Type }\end{array}$} & \multicolumn{3}{|c|}{ Coordinates (Angstroms) } \\
\hline & & & $\mathrm{X}$ & Y & Z \\
\hline . & & & ---------- & ---------- & ---------- \\
\hline 1 & 46 & 0 & -1.714958 & 0.091202 & 0.104249 \\
\hline 2 & 6 & 0 & -4.523148 & 1.131555 & 0.053339 \\
\hline 3 & 6 & 0 & -4.666235 & -0.305885 & -0.239499 \\
\hline 4 & 6 & 0 & 0.593554 & -1.305496 & -1.222263 \\
\hline 5 & 6 & 0 & 0.251157 & 0.624010 & 0.420241 \\
\hline 6 & 6 & 0 & 0.178008 & -0.772341 & 0.109351 \\
\hline 7 & 1 & 0 & 0.050100 & -2.222813 & -1.473854 \\
\hline 8 & 1 & 0 & 0.401473 & 0.953509 & 1.450607 \\
\hline 9 & 1 & 0 & 0.248041 & -1.500657 & 0.922227 \\
\hline 10 & 1 & 0 & 0.604740 & 1.332756 & -0.333177 \\
\hline 11 & 1 & 0 & -3.270016 & 2.554707 & 0.455251 \\
\hline 12 & 1 & 0 & -3.728053 & -1.979789 & -0.491439 \\
\hline 13 & 1 & 0 & 0.438844 & -0.565282 & -2.015075 \\
\hline 14 & 8 & 0 & 2.025743 & -1.645624 & -1.191707 \\
\hline 15 & 1 & 0 & 2.265705 & -2.114917 & -2.005772 \\
\hline 16 & 1 & 0 & 6.058268 & 2.026100 & -1.983812 \\
\hline 17 & 6 & 0 & 5.716948 & 1.733554 & -0.994730 \\
\hline 18 & 6 & 0 & 4.518586 & 1.173581 & -0.811453 \\
\hline 19 & 1 & 0 & 6.392144 & 1.939161 & -0.165651 \\
\hline 20 & 1 & 0 & 3.856028 & 1.002980 & -1.659505 \\
\hline 21 & 6 & 0 & 3.993546 & 0.786450 & 0.535894 \\
\hline 22 & 1 & 0 & 4.673586 & 1.090073 & 1.337535 \\
\hline 23 & 1 & 0 & 3.001683 & 1.208712 & 0.728238 \\
\hline 24 & 7 & 0 & 3.821961 & -0.707894 & 0.632941 \\
\hline 25 & 1 & 0 & 4.709814 & -1.149998 & 0.377321 \\
\hline 26 & 1 & 0 & 3.117628 & -1.028184 & -0.090451 \\
\hline 27 & 6 & 0 & 3.390768 & -1.186043 & 1.974887 \\
\hline 28 & 1 & 0 & 3.275041 & -2.270747 & 1.942064 \\
\hline 29 & 1 & 0 & 4.140668 & -0.908104 & 2.718222 \\
\hline 30 & 1 & 0 & 2.432433 & -0.722183 & 2.210320 \\
\hline 31 & 7 & 0 & -3.582266 & -0.990064 & -0.294925 \\
\hline 32 & 7 & 0 & -3.330318 & 1.556554 & 0.257071 \\
\hline 33 & 1 & 0 & -5.660775 & -0.729917 & -0.393027 \\
\hline 34 & 1 & 0 & -5.409964 & 1.767785 & 0.087948 \\
\hline
\end{tabular}

\begin{tabular}{lrrrr} 
& & 1 & \multicolumn{1}{c}{2} & \multicolumn{1}{c}{3} \\
& & A & \multicolumn{1}{c}{ A } & A \\
Frequencies -- & 24.3008 & 28.4659 & 39.6340 \\
Red. masses -- & 4.3755 & 4.6119 & 4.2425 \\
Frc consts -- & 0.0015 & 0.0022 & 0.0039 \\
IR Inten -- & 0.3201 & 6.1958 & 5.8858
\end{tabular}




\begin{tabular}{|c|c|c|c|c|c|c|c|c|c|c|}
\hline Atom & AN & X & Y & Z & X & Y & Z & X & Y & Z \\
\hline 1 & 46 & -0.03 & 0.00 & 0.00 & -0.01 & -0.03 & 0.07 & -0.02 & 0.01 & 0.07 \\
\hline 2 & 6 & -0.07 & -0.09 & 0.07 & 0.02 & 0.04 & -0.13 & -0.03 & -0.02 & -0.26 \\
\hline 3 & 6 & -0.02 & -0.09 & 0.03 & 0.01 & 0.08 & -0.29 & 0.00 & -0.06 & -0.06 \\
\hline 4 & 6 & 0.00 & 0.10 & -0.05 & 0.00 & 0.06 & 0.03 & -0.06 & 0.02 & 0.04 \\
\hline 5 & 6 & -0.04 & 0.05 & 0.00 & -0.02 & -0.08 & 0.18 & -0.02 & 0.08 & -0.03 \\
\hline 6 & 6 & -0.01 & 0.06 & -0.03 & -0.02 & -0.05 & 0.06 & 0.00 & 0.06 & 0.04 \\
\hline 7 & 1 & 0.02 & 0.09 & -0.07 & -0.01 & 0.09 & -0.06 & -0.06 & 0.00 & 0.11 \\
\hline 8 & 1 & -0.04 & 0.03 & 0.00 & -0.04 & -0.17 & 0.22 & 0.01 & 0.13 & -0.05 \\
\hline 9 & 1 & 0.01 & 0.04 & -0.05 & -0.05 & -0.12 & 0.00 & 0.05 & 0.10 & 0.07 \\
\hline 10 & 1 & -0.06 & 0.08 & 0.01 & 0.01 & -0.02 & 0.25 & -0.06 & 0.05 & -0.07 \\
\hline 11 & 1 & -0.11 & -0.06 & 0.08 & 0.00 & -0.03 & 0.19 & -0.07 & 0.03 & -0.31 \\
\hline 12 & 1 & 0.03 & -0.05 & -0.03 & 0.00 & 0.07 & -0.31 & 0.03 & -0.10 & 0.27 \\
\hline 13 & 1 & -0.02 & 0.11 & -0.03 & 0.02 & 0.13 & 0.08 & -0.12 & -0.02 & 0.02 \\
\hline 14 & 8 & 0.01 & 0.13 & -0.06 & -0.01 & 0.03 & 0.02 & -0.05 & 0.04 & -0.03 \\
\hline 15 & 1 & 0.02 & 0.15 & -0.06 & -0.01 & 0.07 & 0.00 & -0.09 & 0.04 & -0.04 \\
\hline 16 & 1 & 0.36 & -0.28 & 0.03 & -0.03 & -0.07 & -0.17 & 0.11 & 0.10 & 0.10 \\
\hline 17 & 6 & 0.27 & -0.21 & 0.02 & 0.01 & -0.04 & -0.15 & 0.09 & 0.04 & 0.07 \\
\hline 18 & 6 & 0.20 & -0.08 & -0.02 & 0.00 & -0.01 & -0.09 & 0.08 & 0.03 & 0.02 \\
\hline 19 & 1 & 0.24 & -0.24 & 0.05 & 0.04 & -0.04 & -0.18 & 0.07 & -0.01 & 0.10 \\
\hline 20 & 1 & 0.24 & -0.05 & -0.06 & -0.03 & -0.02 & -0.06 & 0.10 & 0.08 & -0.01 \\
\hline 21 & 6 & 0.09 & 0.02 & -0.04 & 0.04 & 0.03 & -0.06 & 0.06 & -0.04 & -0.01 \\
\hline 22 & 1 & 0.06 & 0.01 & -0.01 & 0.08 & 0.04 & -0.09 & 0.04 & -0.09 & 0.01 \\
\hline 23 & 1 & 0.11 & 0.10 & -0.12 & 0.06 & 0.05 & -0.03 & 0.05 & -0.05 & -0.01 \\
\hline 24 & 7 & -0.02 & 0.03 & 0.02 & 0.02 & 0.04 & -0.02 & 0.05 & -0.05 & -0.10 \\
\hline 25 & 1 & -0.04 & -0.04 & 0.07 & 0.01 & 0.02 & -0.02 & 0.04 & -0.04 & -0.15 \\
\hline 26 & 1 & -0.01 & 0.05 & 0.00 & 0.01 & 0.03 & 0.00 & 0.02 & 0.00 & -0.09 \\
\hline 27 & 6 & -0.10 & 0.12 & 0.02 & 0.04 & 0.08 & 0.00 & 0.09 & -0.12 & -0.11 \\
\hline 28 & 1 & -0.17 & 0.13 & 0.06 & 0.04 & 0.08 & 0.04 & 0.06 & -0.12 & -0.18 \\
\hline 29 & 1 & -0.11 & 0.10 & 0.04 & 0.04 & 0.10 & -0.01 & 0.13 & -0.19 & -0.13 \\
\hline 30 & 1 & -0.08 & 0.20 & -0.04 & 0.03 & 0.08 & -0.01 & 0.11 & -0.11 & -0.04 \\
\hline 31 & 7 & 0.00 & -0.05 & -0.01 & 0.00 & 0.05 & -0.20 & 0.01 & -0.06 & 0.12 \\
\hline 32 & 7 & -0.08 & -0.06 & 0.06 & 0.00 & -0.01 & 0.07 & -0.05 & 0.01 & -0.17 \\
\hline 33 & 1 & -0.01 & -0.12 & 0.04 & 0.02 & 0.12 & -0.47 & 0.01 & -0.09 & -0.07 \\
\hline 34 & 1 & -0.09 & -0.13 & 0.10 & 0.03 & 0.07 & -0.17 & -0.03 & -0.01 & -0.46 \\
\hline
\end{tabular}

thermodynamics:

Sum of electronic and zero-point Energies=

$-720.506152$

Sum of electronic and thermal Enthalpies=

$-720.486048$

Sum of electronic and thermal Free Energies=

$-720.556987$

Total free energy in solution:

with all non electrostatic terms (a.u.) $=\quad-720.858602$

Optimized geometry, three lower frequencies, thermochemistry and PCM energy for $\mathbf{T S} \mathbf{S}_{\mathbf{1 1 b}-\mathbf{b} \text {. }}$. 


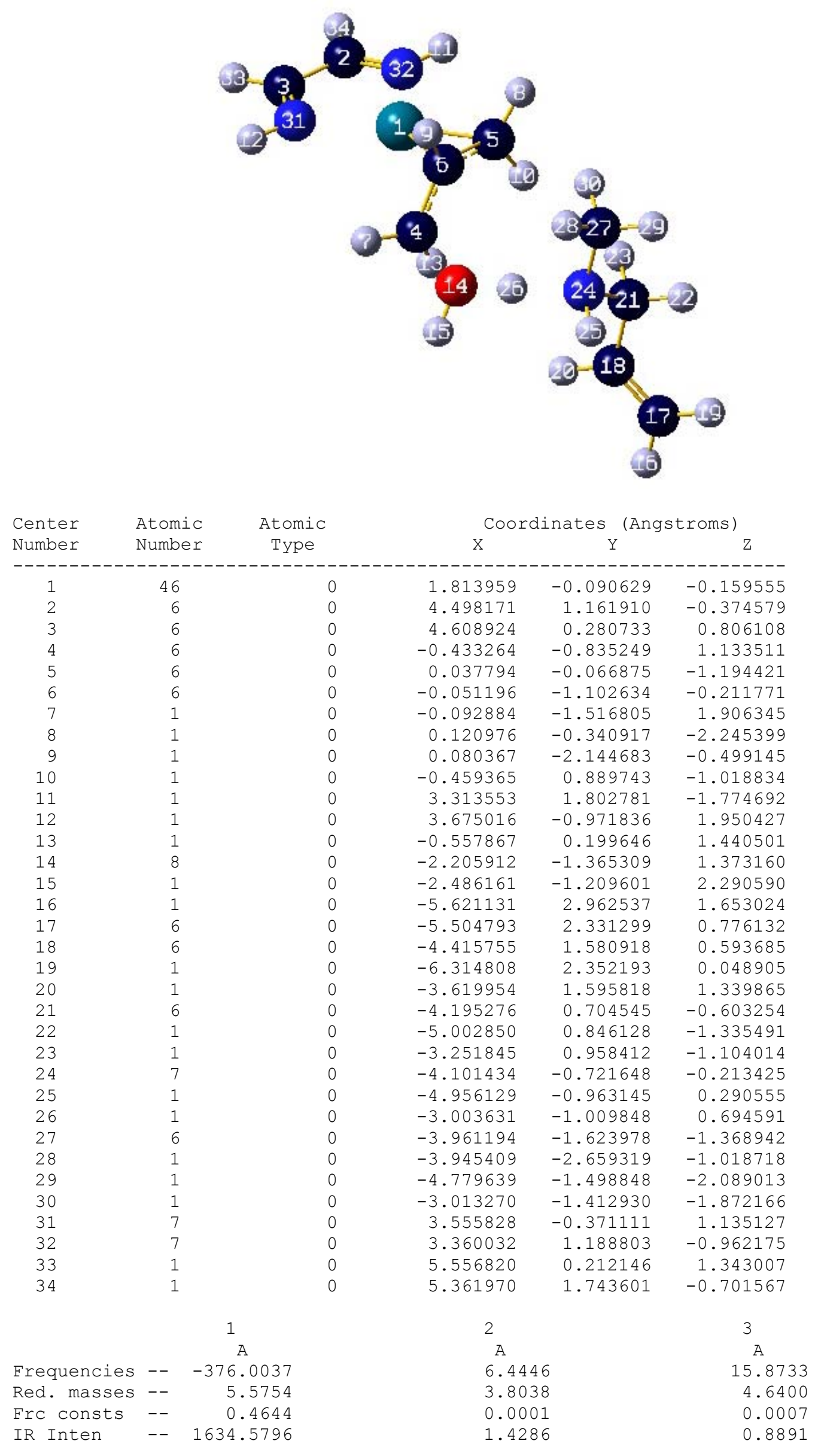




\begin{tabular}{|c|c|c|c|c|c|c|c|c|c|c|}
\hline Atom & AN & X & $\mathrm{Y}$ & Z & X & $\mathrm{Y}$ & Z & X & Y & Z \\
\hline 1 & 46 & 0.00 & 0.00 & 0.02 & -0.02 & 0.04 & 0.00 & -0.03 & -0.02 & 0.00 \\
\hline 2 & 6 & -0.02 & 0.01 & -0.01 & 0.01 & -0.01 & 0.05 & -0.09 & 0.13 & 0.03 \\
\hline 3 & 6 & 0.00 & 0.00 & -0.01 & 0.03 & -0.15 & -0.06 & -0.07 & 0.15 & 0.05 \\
\hline 4 & 6 & 0.47 & 0.10 & -0.08 & 0.02 & -0.04 & 0.01 & -0.02 & -0.08 & -0.02 \\
\hline 5 & 6 & 0.03 & 0.02 & -0.05 & -0.05 & 0.19 & 0.07 & -0.02 & -0.10 & -0.03 \\
\hline 6 & 6 & -0.01 & -0.01 & -0.09 & -0.04 & 0.09 & -0.04 & 0.01 & -0.09 & -0.01 \\
\hline 7 & 1 & 0.14 & -0.04 & -0.08 & 0.03 & -0.12 & -0.08 & 0.00 & -0.06 & -0.01 \\
\hline 8 & 1 & 0.02 & 0.04 & -0.06 & -0.10 & 0.30 & 0.04 & 0.01 & -0.12 & -0.02 \\
\hline 9 & 1 & 0.02 & -0.01 & -0.07 & -0.08 & 0.12 & -0.15 & 0.05 & -0.09 & 0.01 \\
\hline 10 & 1 & 0.04 & 0.01 & -0.01 & -0.02 & 0.19 & 0.19 & -0.05 & -0.12 & -0.05 \\
\hline 11 & 1 & -0.03 & -0.01 & -0.01 & -0.02 & 0.18 & 0.16 & -0.10 & 0.04 & 0.00 \\
\hline 12 & 1 & -0.02 & -0.01 & -0.01 & 0.04 & -0.25 & -0.16 & -0.02 & 0.11 & 0.04 \\
\hline 13 & 1 & 0.16 & 0.04 & -0.06 & 0.05 & -0.06 & 0.12 & -0.05 & -0.08 & -0.03 \\
\hline 14 & 8 & -0.16 & -0.14 & 0.21 & 0.01 & -0.02 & 0.04 & 0.00 & -0.14 & -0.04 \\
\hline 15 & 1 & -0.31 & -0.02 & 0.13 & 0.03 & -0.01 & 0.04 & 0.00 & -0.19 & -0.03 \\
\hline 16 & 1 & 0.00 & -0.02 & 0.02 & 0.14 & 0.11 & -0.16 & 0.38 & 0.16 & 0.01 \\
\hline 17 & 6 & 0.00 & 0.01 & 0.01 & 0.10 & 0.05 & -0.13 & 0.30 & 0.14 & 0.01 \\
\hline 18 & 6 & -0.01 & 0.00 & -0.02 & 0.07 & 0.00 & -0.10 & 0.22 & 0.03 & -0.0 \\
\hline 19 & 1 & 0.00 & 0.03 & 0.00 & 0.09 & 0.03 & -0.11 & 0.29 & 0.22 & 0.03 \\
\hline 20 & 1 & -0.02 & -0.06 & -0.01 & 0.09 & 0.02 & -0.11 & 0.24 & -0.04 & -0.0 \\
\hline 21 & 6 & -0.04 & 0.01 & -0.04 & 0.02 & -0.09 & -0.04 & 0.12 & 0.00 & 0.0 \\
\hline 22 & 1 & -0.01 & 0.14 & -0.07 & 0.01 & -0.11 & -0.04 & 0.11 & 0.07 & 0.0 \\
\hline 23 & 1 & -0.01 & -0.01 & -0.01 & 0.02 & -0.15 & -0.08 & 0.12 & -0.08 & -0.0 \\
\hline 24 & 7 & -0.14 & 0.04 & -0.08 & -0.01 & -0.06 & 0.05 & 0.00 & 0.00 & 0.0 \\
\hline 25 & 1 & -0.17 & 0.05 & -0.14 & -0.01 & -0.01 & 0.08 & -0.01 & 0.07 & 0.0 \\
\hline 26 & 1 & 0.28 & -0.20 & 0.45 & 0.00 & -0.03 & 0.04 & 0.00 & -0.08 & -0.0 \\
\hline 27 & 6 & -0.04 & 0.03 & -0.02 & -0.05 & -0.14 & 0.11 & -0.09 & -0.02 & 0.0 \\
\hline 28 & 1 & -0.04 & 0.03 & -0.01 & -0.07 & -0.12 & 0.17 & -0.17 & -0.02 & 0.0 \\
\hline 29 & 1 & 0.04 & -0.04 & -0.13 & -0.06 & -0.17 & 0.11 & -0.09 & 0.04 & 0.0 \\
\hline 30 & 1 & 0.00 & 0.01 & 0.03 & -0.05 & -0.19 & 0.08 & -0.08 & -0.10 & -0.0 \\
\hline 31 & 7 & -0.01 & 0.02 & 0.00 & 0.02 & -0.16 & -0.09 & -0.04 & 0.09 & 0.0 \\
\hline 32 & 7 & -0.01 & -0.03 & -0.03 & -0.01 & 0.08 & 0.09 & -0.08 & 0.05 & 0.0 \\
\hline 33 & 1 & 0.00 & -0.02 & -0.02 & 0.05 & -0.24 & -0.10 & -0.08 & 0.21 & 0.0 \\
\hline 34 & 1 & -0.04 & 0.05 & 0.01 & 0.01 & 0.00 & 0.08 & -0.12 & 0.17 & 0.0 \\
\hline
\end{tabular}

thermodynamics:

Sum of electronic and zero-point Energies=

$-720.488504$

Sum of electronic and thermal Enthalpies=

$-720.468628$

Sum of electronic and thermal Free Energies=

$-720.540899$

Total free energy in solution:

with all non electrostatic terms (a.u.) $=-720.829686$

Optimized geometry, three lower frequencies, thermochemistry and PCM energy for $\mathbf{1 2 b}$.

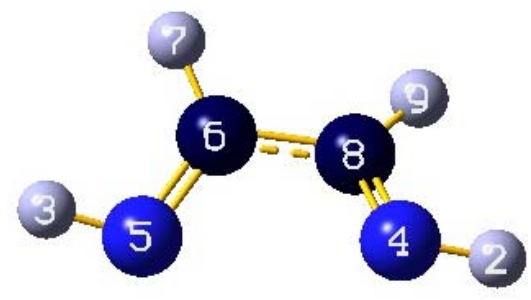

1

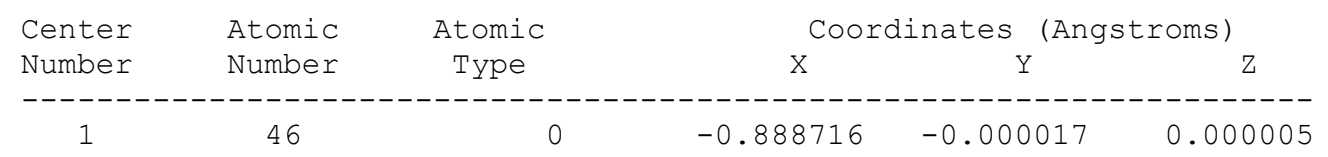




\begin{tabular}{|c|c|c|c|c|c|c|c|c|c|c|}
\hline 2 & & 1 & & 0 & \multicolumn{2}{|c|}{0.826509} & -2.324490 & \multicolumn{3}{|c|}{-0.175790} \\
\hline 3 & & 1 & & 0 & \multicolumn{2}{|c|}{0.826239} & 2.32448 & \multicolumn{3}{|c|}{0.175707} \\
\hline 4 & & 7 & & 0 & \multicolumn{2}{|c|}{0.772967} & -1.35206 & \multicolumn{3}{|c|}{0.135898} \\
\hline 5 & & 7 & & 0 & \multicolumn{2}{|c|}{0.772823} & 1.35204 & \multicolumn{3}{|c|}{-0.135957} \\
\hline 6 & & 6 & & 0 & \multicolumn{2}{|c|}{1.899096} & 0.71240 & \multicolumn{3}{|c|}{0.088746} \\
\hline 7 & & 1 & & 0 & \multicolumn{2}{|c|}{2.808935} & 1.22584 & \multicolumn{3}{|c|}{0.406904} \\
\hline 8 & & 6 & & 0 & \multicolumn{2}{|c|}{1.899178} & -0.71229 & \multicolumn{3}{|c|}{-0.088717} \\
\hline 9 & & 1 & & 0 & \multicolumn{2}{|c|}{2.809101} & -1.22562 & \multicolumn{2}{|c|}{-0.406806} & \\
\hline & & \multicolumn{3}{|c|}{1} & \multicolumn{3}{|c|}{2} & \multicolumn{3}{|c|}{3} \\
\hline & & \multicolumn{3}{|c|}{ A } & \multicolumn{3}{|c|}{ A } & \multicolumn{3}{|c|}{ A } \\
\hline \multicolumn{2}{|c|}{ Frequencies } & j-- & \multicolumn{2}{|c|}{153.2699} & \multicolumn{3}{|c|}{225.5942} & \multicolumn{3}{|c|}{228.4459} \\
\hline Red. & masses & -- & \multicolumn{2}{|c|}{4.0804} & \multicolumn{3}{|c|}{5.7816} & \multicolumn{3}{|c|}{9.2133} \\
\hline $\operatorname{Frc}$ & consts & -- & \multicolumn{2}{|c|}{0.0565} & \multirow{2}{*}{\multicolumn{3}{|c|}{0.1734}} & & 0.283 & \\
\hline IR $I_{1}$ & nten & -- & 8.18 & & & 0.378 & & & 10.695 & \\
\hline Atom & AN & $\mathrm{x}$ & $\mathrm{Y}$ & Z & $\mathrm{x}$ & Y & Z & $\mathrm{x}$ & Y & Z \\
\hline 1 & 46 & 0.00 & 0.04 & 0.03 & 0.00 & 0.07 & -0.02 & 0.17 & 0.00 & 0.00 \\
\hline 2 & 1 & 0.21 & -0.08 & -0.35 & 0.37 & -0.22 & 0.21 & -0.34 & -0.01 & -0.18 \\
\hline 3 & 1 & -0.21 & -0.08 & -0.35 & -0.36 & -0.22 & 0.21 & -0.35 & 0.00 & 0.19 \\
\hline 4 & 7 & 0.09 & -0.12 & -0.25 & 0.26 & -0.21 & 0.18 & -0.31 & -0.07 & 0.04 \\
\hline 5 & 7 & -0.08 & -0.12 & -0.25 & -0.25 & -0.22 & 0.18 & -0.32 & 0.06 & -0.03 \\
\hline 6 & 6 & -0.09 & -0.02 & 0.15 & -0.09 & -0.05 & -0.10 & -0.33 & -0.01 & 0.00 \\
\hline 7 & 1 & -0.23 & 0.08 & 0.39 & -0.12 & 0.11 & -0.29 & -0.35 & -0.01 & 0.03 \\
\hline 8 & 6 & 0.09 & -0.02 & 0.15 & 0.10 & -0.05 & -0.10 & -0.33 & 0.01 & 0.00 \\
\hline 9 & 1 & 0.23 & 0.08 & 0.39 & 0.13 & 0.11 & -0.28 & -0.34 & 0.02 & -0.04 \\
\hline
\end{tabular}

thermodynamics:

Sum of electronic and zero-point Energies=

$-314.728605$

Sum of electronic and thermal Enthalpies=

$-314.722119$

Sum of electronic and thermal Free Energies=

$-314.758866$

Total free energy in solution:

with all non electrostatic terms (a.u.) $=\quad-314.800226$

Optimized geometry, three lower frequencies, thermochemistry and PCM energy for $\mathbf{1 3 b}$.

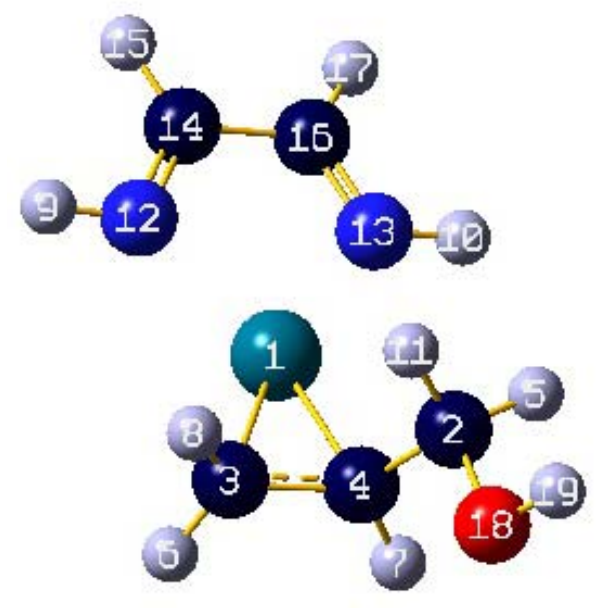

\begin{tabular}{|c|c|c|c|c|c|}
\hline \multirow{2}{*}{$\begin{array}{l}\text { Center } \\
\text { Number }\end{array}$} & \multirow{2}{*}{$\begin{array}{l}\text { Atomic } \\
\text { Number }\end{array}$} & \multirow{2}{*}{$\begin{array}{c}\text { Atomic } \\
\text { Type }\end{array}$} & \multicolumn{3}{|c|}{ Coordinates (Angstroms) } \\
\hline & & & $\mathrm{x}$ & $\mathrm{Y}$ & Z \\
\hline 1 & 46 & 0 & -0.197786 & -0.265731 & -0.234750 \\
\hline 2 & 6 & 0 & 2.559435 & 0.793696 & 0.513775 \\
\hline 3 & 6 & 0 & 1.496993 & -1.459063 & -0.065553 \\
\hline 4 & 6 & 0 & 1.885704 & -0.148183 & -0.441995 \\
\hline 5 & 1 & 0 & 2.272511 & 1.833340 & 0.291816 \\
\hline
\end{tabular}




\begin{tabular}{|c|c|c|c|c|c|}
\hline 6 & 1 & 0 & 1.478897 & -2.256186 & -0.810169 \\
\hline 7 & 1 & 0 & 2.138163 & 0.035782 & -1.489537 \\
\hline 8 & 1 & 0 & 1.668538 & -1.803312 & 0.956176 \\
\hline 9 & 1 & 0 & -2.374849 & -1.879208 & 0.905695 \\
\hline 10 & 1 & 0 & -1.461180 & 2.334150 & -0.828433 \\
\hline 11 & 1 & 0 & 2.250609 & 0.567451 & 1.545765 \\
\hline 12 & 7 & 0 & -2.143638 & -0.975205 & 0.495484 \\
\hline 13 & 7 & 0 & -1.598467 & 1.417240 & -0.404553 \\
\hline 14 & 6 & 0 & -3.058735 & -0.080882 & 0.648991 \\
\hline 15 & 1 & 0 & -4.006088 & -0.259373 & 1.163799 \\
\hline 16 & 6 & 0 & -2.779677 & 1.237927 & 0.077529 \\
\hline 17 & 1 & 0 & -3.561790 & 2.001212 & 0.069614 \\
\hline 18 & 8 & 0 & 3.981859 & 0.647533 & 0.378316 \\
\hline 19 & 1 & 0 & 4.410884 & 1.314295 & 0.934245 \\
\hline
\end{tabular}

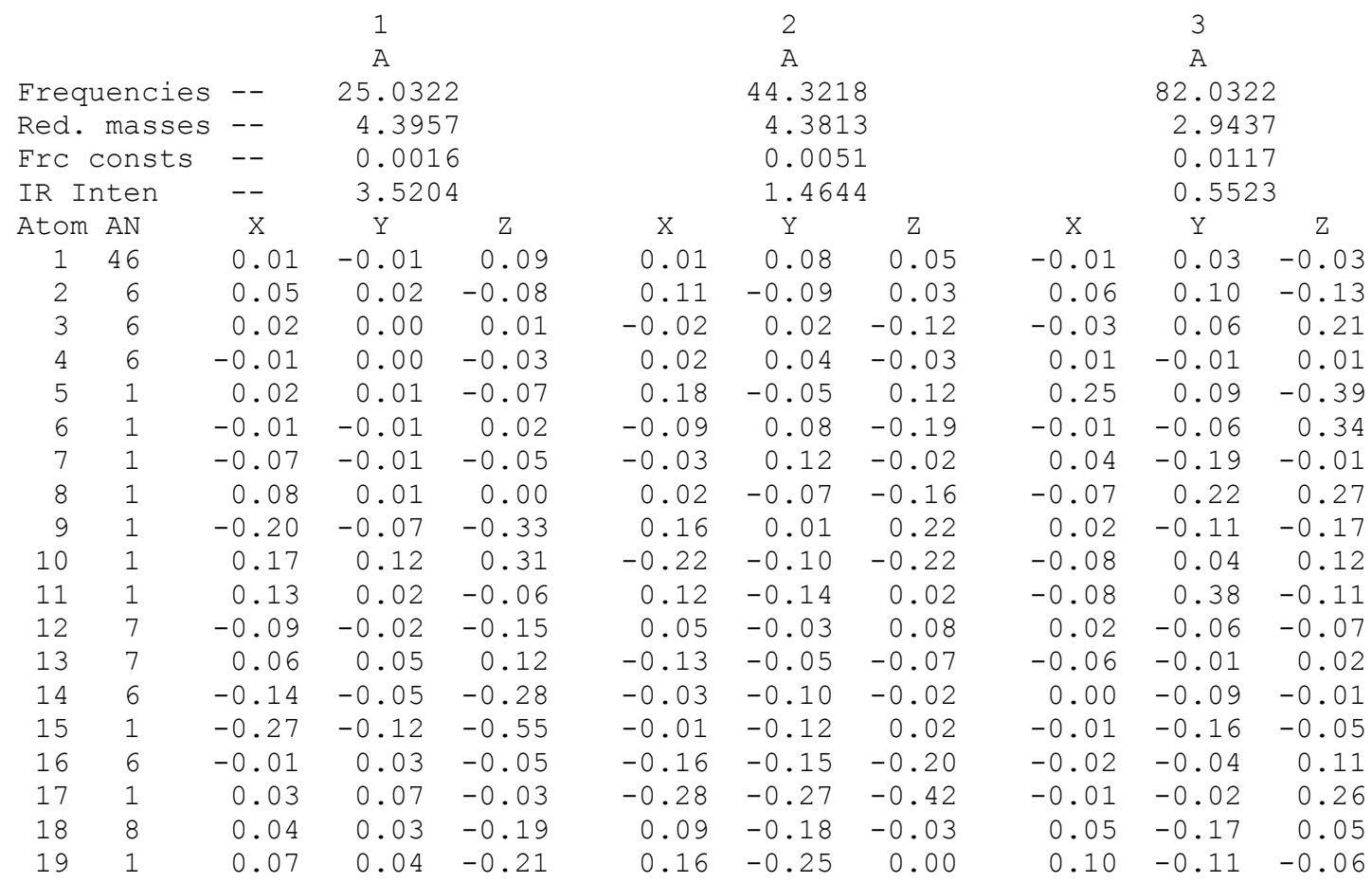

thermodynamics:

Sum of electronic and zero-point Energies=

$-507.746382$

Sum of electronic and thermal Enthalpies=

$-507.734094$

Sum of electronic and thermal Free Energies=

$-507.785546$

Total free energy in solution:

with all non electrostatic terms

$(\mathrm{a} \cdot \mathrm{u})=$.

\section{III. $\mathrm{PH}_{3}$}

Optimized geometry, three lower frequencies and PCM energy for 1c. 


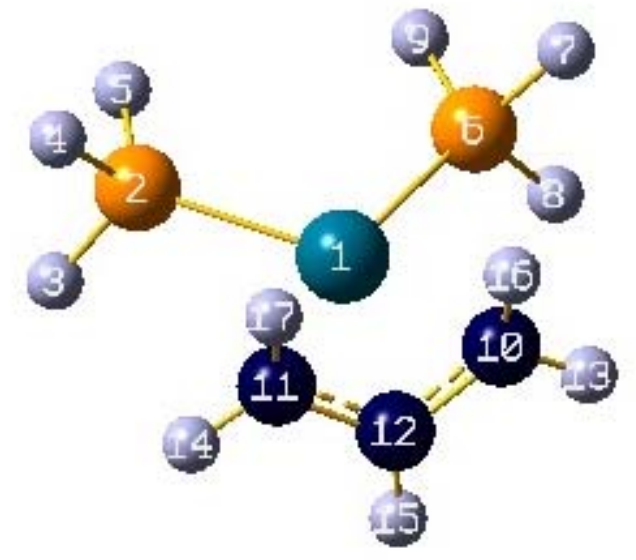

\begin{tabular}{|c|c|c|c|c|c|}
\hline \multirow{2}{*}{$\begin{array}{l}\text { Center } \\
\text { Number }\end{array}$} & \multirow{2}{*}{$\begin{array}{l}\text { Atomic } \\
\text { Number }\end{array}$} & \multirow{2}{*}{$\begin{array}{l}\text { Atomic } \\
\text { Type }\end{array}$} & \multicolumn{3}{|c|}{ Coordinates (Angstroms) } \\
\hline & & & $\mathrm{X}$ & $\mathrm{Y}$ & Z \\
\hline 1 & 46 & 0 & 0.103756 & -0.000001 & 0.010722 \\
\hline 2 & 15 & 0 & -1.371487 & -1.820919 & -0.020022 \\
\hline 3 & 1 & 0 & -1.279160 & -2.702263 & -1.114803 \\
\hline 4 & 1 & 0 & -1.275602 & -2.746377 & 1.037598 \\
\hline 5 & 1 & 0 & -2.764185 & -1.606611 & -0.013805 \\
\hline 6 & 15 & 0 & -1.371468 & 1.820932 & -0.020022 \\
\hline 7 & 1 & 0 & -1.275609 & 2.746358 & 1.037628 \\
\hline 8 & 1 & 0 & -1.279096 & 2.702308 & -1.114774 \\
\hline 9 & 1 & 0 & -2.764168 & 1.606638 & -0.013858 \\
\hline 10 & 6 & 0 & 1.920411 & 1.213705 & 0.204309 \\
\hline 11 & 6 & 0 & 1.920401 & -1.213722 & 0.204309 \\
\hline 12 & 6 & 0 & 2.252514 & -0.000010 & -0.429165 \\
\hline 13 & 1 & 0 & 2.022280 & 2.148336 & -0.340817 \\
\hline 14 & 1 & 0 & 2.022262 & -2.148354 & -0.340817 \\
\hline 15 & 1 & 0 & 2.502545 & -0.000011 & -1.489218 \\
\hline 16 & 1 & 0 & 1.951181 & 1.291616 & 1.291799 \\
\hline 17 & 1 & 0 & 1.951169 & -1.291633 & 1.291799 \\
\hline
\end{tabular}

\begin{tabular}{|c|c|c|c|c|c|c|c|c|c|c|}
\hline & & & 1 & & & 2 & & & 3 & \\
\hline & & & A & & & A & & & A & \\
\hline Frequ & uencies & -- & 39.907 & & & 44.05 & & & 96.950 & \\
\hline Red. & masses & -- & 1.022 & & & 1.02 & & & 2.634 & \\
\hline Frc o & consts & -- & 0.001 & & & 0.00 & & & 0.014 & \\
\hline IR In & nten & -- & 0.328 & & & 0.00 & & & 0.096 & \\
\hline Atom & AN & $\mathrm{X}$ & Y & Z & $\mathrm{X}$ & $\mathrm{Y}$ & Z & $\mathrm{X}$ & Y & Z \\
\hline 1 & 46 & 0.00 & 0.00 & 0.00 & 0.00 & 0.00 & 0.00 & 0.00 & 0.00 & 0.00 \\
\hline 2 & 15 & 0.00 & 0.00 & 0.01 & 0.00 & 0.00 & 0.01 & 0.00 & 0.00 & 0.08 \\
\hline 3 & 1 & 0.25 & -0.25 & 0.23 & -0.26 & 0.24 & -0.21 & -0.11 & -0.03 & 0.10 \\
\hline 4 & 1 & -0.26 & 0.24 & 0.24 & 0.26 & -0.23 & -0.22 & 0.11 & 0.03 & 0.10 \\
\hline 5 & 1 & 0.00 & 0.01 & -0.37 & 0.00 & -0.01 & 0.39 & 0.00 & 0.00 & 0.22 \\
\hline 6 & 15 & 0.00 & 0.00 & 0.01 & 0.00 & 0.00 & -0.01 & 0.00 & 0.00 & -0.08 \\
\hline 7 & 1 & -0.26 & -0.24 & 0.24 & -0.26 & -0.23 & 0.22 & -0.11 & 0.03 & -0.10 \\
\hline 8 & 1 & 0.25 & 0.25 & 0.23 & 0.26 & 0.24 & 0.21 & 0.11 & -0.03 & -0.10 \\
\hline 9 & 1 & 0.00 & -0.01 & -0.37 & 0.00 & -0.01 & -0.39 & 0.00 & 0.00 & -0.22 \\
\hline 10 & 6 & 0.00 & 0.00 & -0.01 & 0.00 & 0.00 & -0.02 & 0.01 & -0.03 & 0.23 \\
\hline 11 & 6 & 0.00 & 0.00 & -0.01 & 0.00 & 0.00 & 0.02 & -0.01 & -0.03 & -0.23 \\
\hline 12 & 6 & 0.00 & 0.00 & -0.01 & 0.00 & -0.01 & 0.00 & 0.00 & 0.09 & 0.00 \\
\hline 13 & 1 & 0.00 & 0.00 & -0.01 & 0.00 & -0.01 & -0.04 & 0.03 & 0.08 & 0.42 \\
\hline 14 & 1 & 0.00 & 0.00 & -0.01 & 0.00 & -0.01 & 0.04 & -0.03 & 0.08 & -0.42 \\
\hline 15 & 1 & 0.00 & 0.00 & -0.01 & 0.00 & -0.03 & 0.00 & 0.00 & 0.29 & 0.00 \\
\hline 16 & 1 & 0.01 & 0.00 & -0.01 & 0.00 & 0.03 & -0.03 & -0.04 & -0.25 & 0.24 \\
\hline 17 & 1 & 0.01 & 0.00 & -0.01 & 0.00 & 0.03 & 0.03 & 0.04 & -0.25 & -0.24 \\
\hline
\end{tabular}

thermodynamics:

Sum of electronic and zero-point Energies=

$-929.964760$ 
Total free energy in solution:

with all non electrostatic terms

$(\mathrm{a} \cdot \mathrm{u} \cdot)=\quad-930.157872$

\section{Optimized geometry, three lower frequencies and PCM energy for $\mathbf{T S}_{\mathbf{1 c 2}}$.}

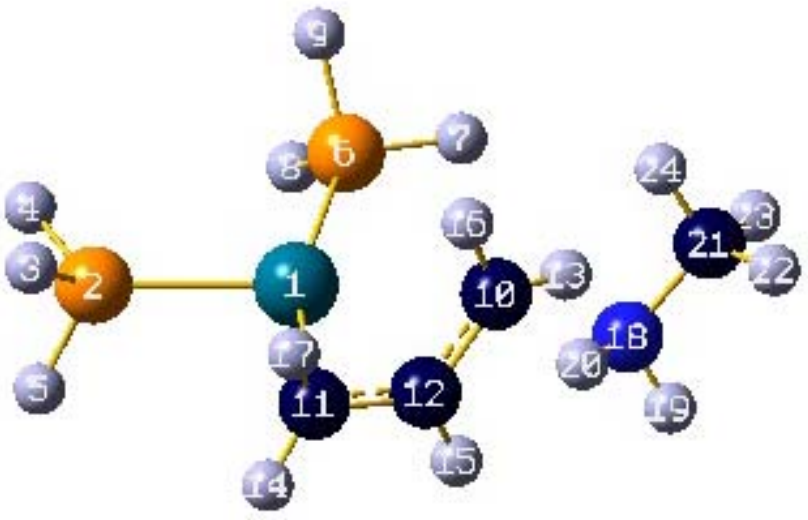

\begin{tabular}{|c|c|c|c|c|c|}
\hline \multirow{2}{*}{$\begin{array}{l}\text { Center } \\
\text { Number }\end{array}$} & \multirow{2}{*}{$\begin{array}{l}\text { Atomic } \\
\text { Number }\end{array}$} & \multirow{2}{*}{$\begin{array}{l}\text { Atomic } \\
\text { Type }\end{array}$} & \multicolumn{3}{|c|}{ Coordinates (Angstroms) } \\
\hline & & & $\mathrm{x}$ & $\mathrm{Y}$ & Z \\
\hline 1 & 46 & 0 & -0.715927 & -0.059435 & -0.099548 \\
\hline 2 & 15 & 0 & -2.744091 & -1.061148 & 0.481462 \\
\hline 3 & 1 & 0 & -2.749650 & -2.019738 & 1.516584 \\
\hline 4 & 1 & 0 & -3.863226 & -0.308704 & 0.899513 \\
\hline 5 & 1 & 0 & -3.381837 & -1.829879 & -0.514661 \\
\hline 6 & 15 & 0 & -1.248656 & 2.241333 & -0.072560 \\
\hline 7 & 1 & 0 & -0.374455 & 3.233524 & -0.569278 \\
\hline 8 & 1 & 0 & -2.410601 & 2.656616 & -0.758038 \\
\hline 9 & 1 & 0 & -1.531752 & 2.843889 & 1.172544 \\
\hline 10 & 6 & 0 & 1.879352 & 0.138345 & -0.110429 \\
\hline 11 & 6 & 0 & 0.414022 & -1.852631 & -0.286692 \\
\hline 12 & 6 & 0 & 1.181354 & -0.814930 & -0.880614 \\
\hline 13 & 1 & 0 & 2.179591 & 1.081604 & -0.552358 \\
\hline 14 & 1 & 0 & -0.009633 & -2.623941 & -0.925553 \\
\hline 15 & 1 & 0 & 1.212336 & -0.725348 & -1.965367 \\
\hline 16 & 1 & 0 & 1.854088 & 0.098321 & 0.974297 \\
\hline 17 & 1 & 0 & 0.628129 & -2.169404 & 0.734945 \\
\hline 18 & 7 & 0 & 3.974878 & -0.428033 & -0.046894 \\
\hline 19 & 1 & 0 & 4.267184 & -0.488908 & -1.020699 \\
\hline 20 & 1 & 0 & 3.979072 & -1.376643 & 0.323537 \\
\hline 21 & 6 & 0 & 4.855492 & 0.456947 & 0.723601 \\
\hline 22 & 1 & 0 & 5.901700 & 0.127330 & 0.716715 \\
\hline 23 & 1 & 0 & 4.807040 & 1.466629 & 0.305913 \\
\hline 24 & 1 & 0 & 4.510402 & 0.495732 & 1.760640 \\
\hline
\end{tabular}

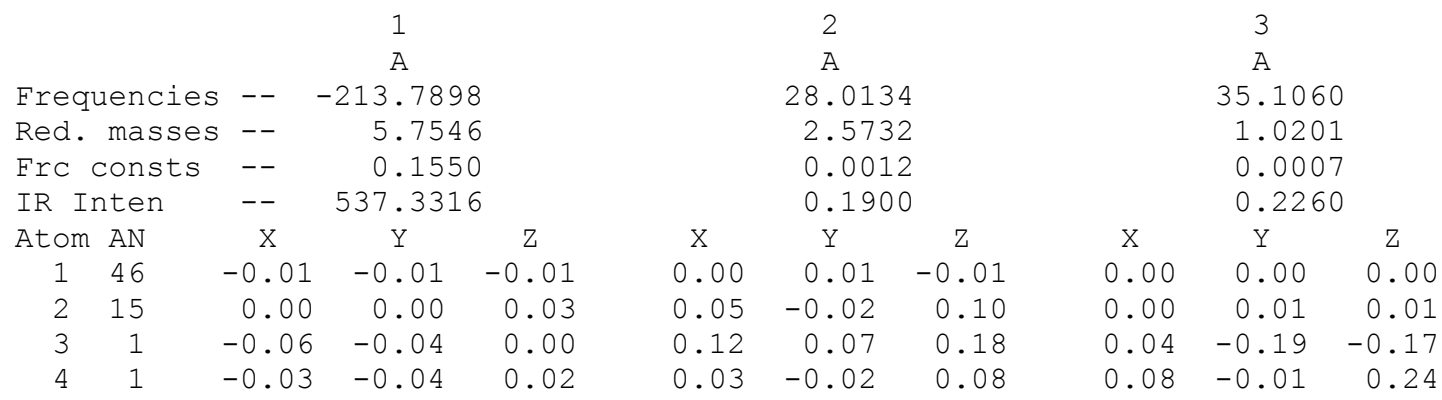




\begin{tabular}{|c|c|c|c|c|c|c|c|c|c|c|}
\hline 5 & 1 & 0.00 & 0.02 & 0.00 & 0.05 & -0.12 & 0.18 & -0.13 & 0.22 & -0.08 \\
\hline 6 & 15 & 0.01 & 0.00 & -0.02 & -0.04 & 0.00 & -0.04 & -0.01 & 0.00 & -0.01 \\
\hline 7 & 1 & -0.03 & 0.06 & 0.02 & -0.03 & 0.02 & 0.01 & 0.18 & 0.05 & 0.43 \\
\hline 8 & 1 & 0.00 & 0.02 & 0.00 & -0.01 & -0.03 & -0.12 & 0.27 & 0.03 & -0.45 \\
\hline 9 & 1 & -0.01 & -0.02 & -0.01 & -0.13 & 0.00 & -0.07 & -0.53 & -0.10 & -0.08 \\
\hline 10 & 6 & 0.48 & -0.08 & 0.10 & -0.01 & 0.04 & -0.07 & 0.00 & -0.01 & 0.00 \\
\hline 11 & 6 & 0.09 & 0.09 & 0.01 & 0.02 & 0.02 & -0.04 & 0.00 & 0.00 & 0.00 \\
\hline 12 & 6 & 0.08 & 0.14 & 0.08 & -0.01 & 0.03 & -0.06 & 0.00 & -0.01 & 0.00 \\
\hline 13 & 1 & 0.21 & 0.05 & 0.17 & -0.02 & 0.04 & -0.08 & 0.00 & -0.01 & 0.00 \\
\hline 14 & 1 & 0.11 & 0.12 & -0.05 & 0.01 & 0.02 & -0.03 & -0.01 & 0.00 & -0.01 \\
\hline 15 & 1 & 0.07 & 0.20 & 0.08 & -0.03 & 0.03 & -0.06 & 0.00 & 0.00 & 0.00 \\
\hline 16 & 1 & 0.21 & 0.00 & 0.10 & 0.01 & 0.04 & -0.07 & 0.00 & -0.01 & 0.00 \\
\hline 17 & 1 & 0.08 & 0.01 & -0.01 & 0.05 & 0.02 & -0.05 & -0.01 & -0.01 & 0.00 \\
\hline 18 & 7 & -0.28 & 0.01 & -0.03 & 0.01 & 0.04 & -0.08 & 0.00 & 0.00 & 0.00 \\
\hline 19 & 1 & -0.35 & 0.01 & -0.06 & 0.09 & 0.28 & -0.07 & 0.00 & -0.02 & 0.00 \\
\hline 20 & 1 & -0.32 & 0.00 & -0.05 & -0.02 & -0.05 & -0.32 & 0.00 & 0.00 & 0.01 \\
\hline 21 & 6 & -0.17 & -0.04 & -0.06 & -0.06 & -0.15 & 0.21 & 0.00 & 0.01 & -0.01 \\
\hline 22 & 1 & -0.21 & -0.15 & -0.16 & -0.05 & -0.14 & 0.20 & 0.00 & 0.01 & -0.01 \\
\hline 23 & 1 & -0.12 & -0.04 & -0.05 & -0.03 & -0.04 & 0.47 & 0.00 & 0.00 & -0.03 \\
\hline 24 & 1 & -0.10 & -0.04 & -0.04 & -0.13 & -0.42 & 0.19 & 0.00 & 0.02 & -0.01 \\
\hline
\end{tabular}

thermodynamics:

Sum of electronic and zero-point Energies=

Sum of electronic and thermal Enthalpies=

Total free energy in solution:

with all non electrostatic terms

$(\mathrm{a} \cdot \mathrm{u})=$.

Optimized geometry, three lower frequencies and PCM energy for 2c.

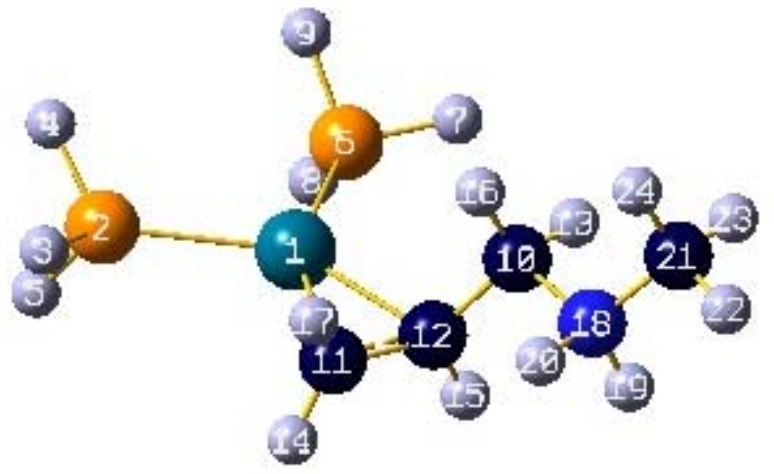

\begin{tabular}{|c|c|c|c|c|c|}
\hline \multirow{2}{*}{$\begin{array}{l}\text { Center } \\
\text { Number }\end{array}$} & \multirow{2}{*}{$\begin{array}{l}\text { Atomic } \\
\text { Number }\end{array}$} & \multirow{2}{*}{$\begin{array}{c}\text { Atomic } \\
\text { Type }\end{array}$} & \multicolumn{3}{|c|}{ Coordinates (Angstroms) } \\
\hline & & & $\mathrm{X}$ & $\mathrm{Y}$ & Z \\
\hline 1 & 46 & 0 & -0.734366 & -0.071820 & -0.124707 \\
\hline 2 & 15 & 0 & -2.658093 & -1.274881 & 0.475056 \\
\hline 3 & 1 & 0 & -2.559794 & -2.617470 & 0.906002 \\
\hline 4 & 1 & 0 & -3.528516 & -0.831752 & 1.497849 \\
\hline 5 & 1 & 0 & -3.645076 & -1.482519 & -0.515458 \\
\hline 6 & 15 & 0 & -1.437829 & 2.169564 & 0.011115 \\
\hline 7 & 1 & 0 & -0.555352 & 3.271980 & -0.095262 \\
\hline 8 & 1 & 0 & -2.369485 & 2.620704 & -0.952139 \\
\hline 9 & 1 & 0 & -2.133154 & 2.635063 & 1.151483 \\
\hline 10 & 6 & 0 & 2.283246 & 0.283858 & 0.158068 \\
\hline 11 & 6 & 0 & 0.701631 & -1.561940 & -0.557627 \\
\hline 12 & 6 & 0 & 1.284509 & -0.285032 & -0.790163 \\
\hline 13 & 1 & 0 & 2.393641 & 1.369560 & 0.088763 \\
\hline 14 & 1 & 0 & 0.386969 & -2.175328 & -1.399820 \\
\hline
\end{tabular}




$\begin{array}{rrrrrr}15 & 1 & 0 & 1.354822 & 0.082970 & -1.815763 \\ 16 & 1 & 0 & 2.090797 & 0.005691 & 1.197839 \\ 17 & 1 & 0 & 0.943416 & -2.124471 & 0.346769 \\ 18 & 7 & 0 & 3.691474 & -0.283633 & -0.148511 \\ 19 & 1 & 0 & 3.917397 & -0.049774 & -1.120727 \\ 20 & 1 & 0 & 3.607731 & -1.305778 & -0.131787 \\ 21 & 6 & 0 & 4.787369 & 0.168798 & 0.756818 \\ 22 & 1 & 0 & 5.728617 & -0.291200 & 0.449441 \\ 23 & 1 & 0 & 4.865119 & 1.255508 & 0.696214 \\ 24 & 1 & 0 & 4.541674 & -0.128408 & 1.777540\end{array}$

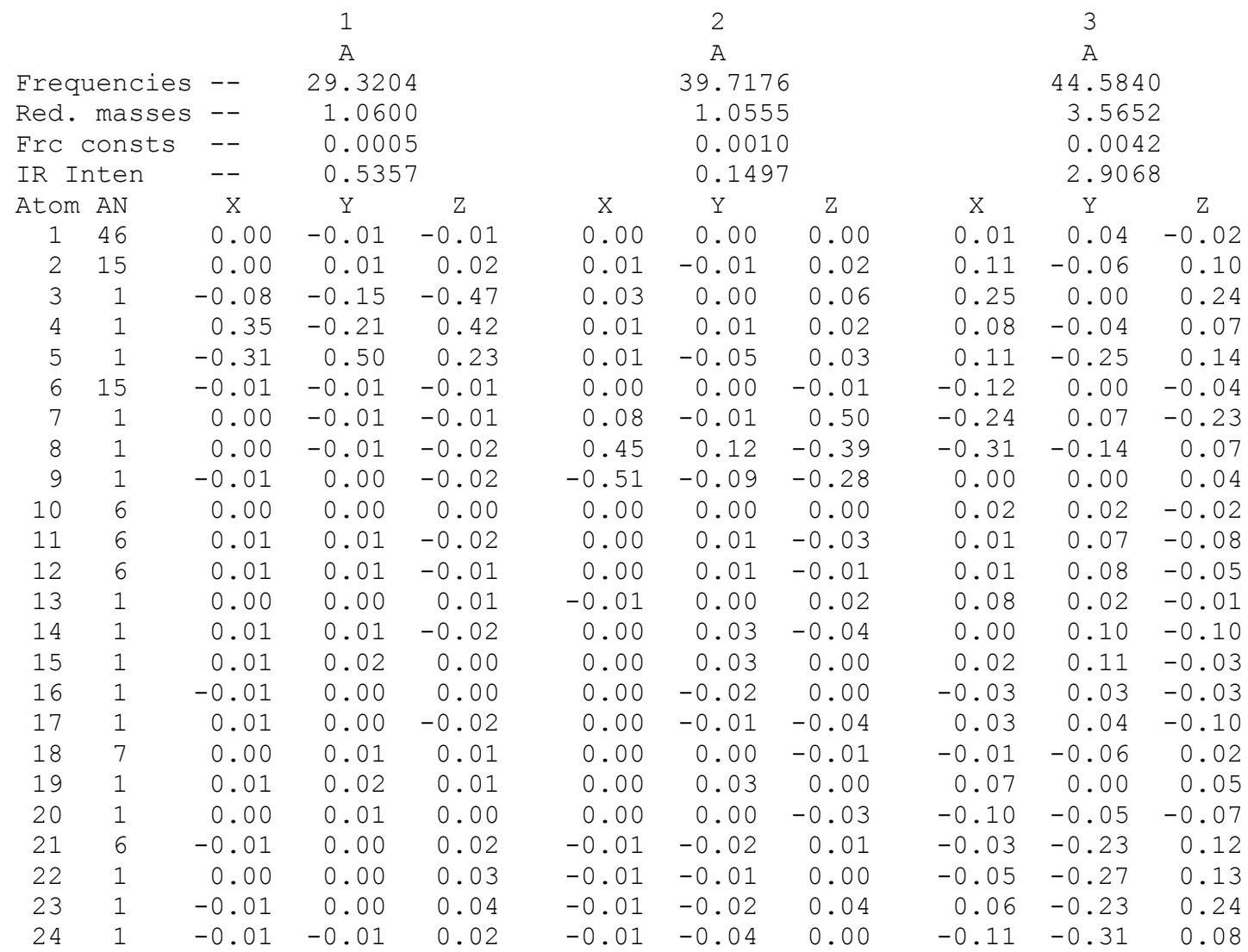

thermodynamics:

Sum of electronic and zero-point Energies=

$-1025.734177$

Sum of electronic and thermal Enthalpies=

$-1025.718321$

Sum of electronic and thermal Free Energies=

$-1025.779055$

Total free energy in solution:

with all non electrostatic terms

$($ a.u. $)=-1025.998789$

Optimized geometry, three lower frequencies and PCM energy for 11c. 


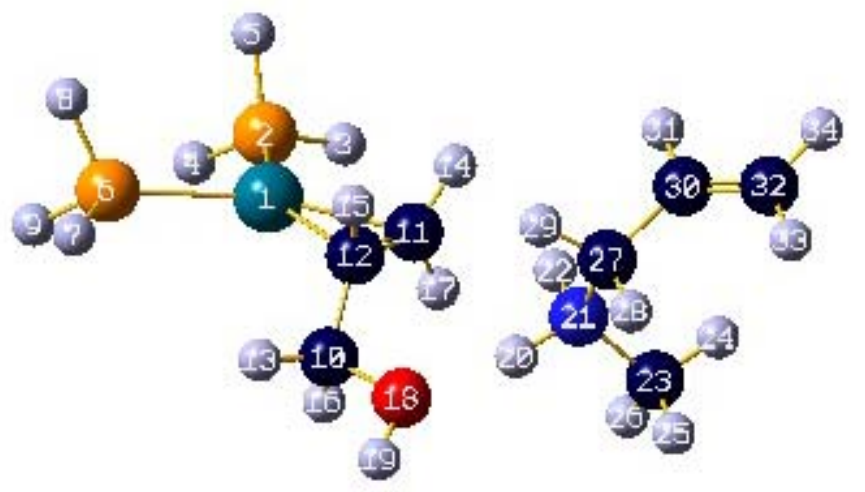

\begin{tabular}{|c|c|c|c|c|c|}
\hline \multirow{2}{*}{$\begin{array}{l}\text { Center } \\
\text { Number }\end{array}$} & \multirow{2}{*}{$\begin{array}{l}\text { Atomic } \\
\text { Number }\end{array}$} & \multirow{2}{*}{$\begin{array}{l}\text { Atomic } \\
\text { Type }\end{array}$} & \multicolumn{3}{|c|}{ Coordinates (Angstroms) } \\
\hline & & & $\mathrm{X}$ & Y & Z \\
\hline 1 & 46 & 0 & -1.812735 & -0.175942 & -0.060764 \\
\hline 2 & 15 & 0 & -3.105222 & -2.046287 & 0.535399 \\
\hline 3 & 1 & 0 & -2.584505 & -3.072047 & 1.359542 \\
\hline 4 & 1 & 0 & -4.332193 & -1.897240 & 1.224363 \\
\hline 5 & 1 & 0 & -3.593554 & -2.893743 & -0.486832 \\
\hline 6 & 15 & 0 & -3.381853 & 1.325725 & -0.941692 \\
\hline 7 & 1 & 0 & -3.064856 & 2.672007 & -1.246390 \\
\hline 8 & 1 & 0 & -3.975121 & 1.019577 & -2.188892 \\
\hline 9 & 1 & 0 & -4.585048 & 1.603144 & -0.250623 \\
\hline 10 & 6 & 0 & 0.267458 & 1.911304 & 0.691480 \\
\hline 11 & 6 & 0 & 0.213498 & -0.628148 & 0.408695 \\
\hline 12 & 6 & 0 & 0.146298 & 0.677225 & -0.149053 \\
\hline 13 & 1 & 0 & -0.269556 & 2.750939 & $0.23820 s$ \\
\hline 14 & 1 & 0 & 0.516626 & -1.480361 & -0.20192 \\
\hline 15 & 1 & 0 & 0.412815 & 0.813570 & -1.19940 \\
\hline 16 & 1 & 0 & -0.118600 & 1.742162 & 1.703408 \\
\hline 17 & 1 & 0 & 0.309321 & -0.768022 & 1.487302 \\
\hline 18 & 8 & 0 & 1.678470 & 2.291657 & 0.78947 \\
\hline 19 & 1 & 0 & 1.754299 & 3.172277 & $1.18797 C$ \\
\hline 20 & 1 & 0 & 2.869056 & 1.085052 & 0.67989 \\
\hline 21 & 7 & 0 & 3.395159 & 0.187873 & 0.52232 \\
\hline 22 & 1 & 0 & 2.630898 & -0.490860 & 0.39403 \\
\hline 23 & 6 & 0 & 4.189355 & -0.166049 & 1.727332 \\
\hline 24 & 1 & 0 & 4.693639 & -1.119425 & 1.563991 \\
\hline 25 & 1 & 0 & 4.928986 & 0.617543 & 1.90275 \\
\hline 26 & 1 & 0 & 3.515428 & -0.235057 & 2.583138 \\
\hline 27 & 6 & 0 & 4.187225 & 0.288881 & -0.75574 \\
\hline 28 & 1 & 0 & 4.979076 & 1.023193 & -0.582168 \\
\hline 29 & 1 & 0 & 3.494513 & 0.698600 & -1.499076 \\
\hline 30 & 6 & 0 & 4.738368 & -1.034609 & -1.185740 \\
\hline 31 & 1 & 0 & 4.009138 & -1.805184 & -1.441102 \\
\hline 32 & 6 & 0 & 6.042570 & -1.282934 & -1.324066 \\
\hline 33 & 1 & 0 & 6.799946 & -0.532020 & -1.105986 \\
\hline 34 & 1 & 0 & 6.399120 & -2.244713 & -1.682147 \\
\hline
\end{tabular}

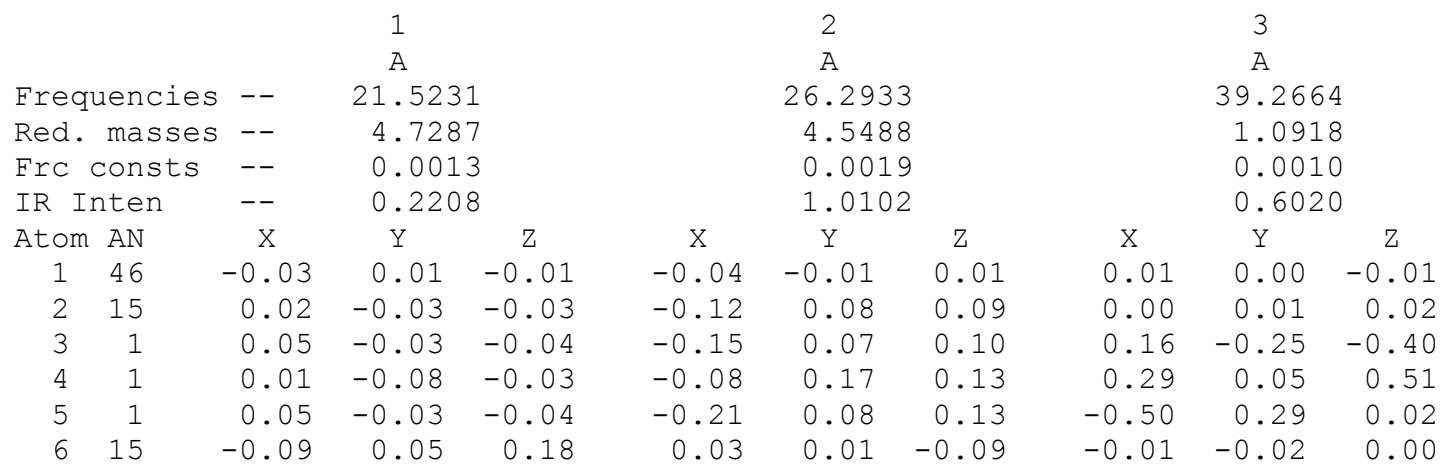




\begin{tabular}{|c|c|c|c|c|c|c|c|c|c|c|}
\hline 7 & 1 & -0.11 & 0.07 & 0.22 & 0.08 & 0.00 & -0.11 & 0.01 & -0.04 & -0.10 \\
\hline 8 & 1 & -0.19 & 0.12 & 0.21 & 0.09 & -0.01 & -0.11 & -0.09 & -0.07 & 0.05 \\
\hline 9 & 1 & -0.04 & 0.02 & 0.29 & 0.01 & 0.06 & -0.15 & 0.04 & 0.06 & 0.05 \\
\hline 10 & 6 & 0.01 & -0.01 & -0.07 & 0.01 & -0.06 & -0.01 & 0.00 & 0.01 & 0.00 \\
\hline 11 & 6 & 0.00 & 0.00 & -0.14 & -0.05 & -0.06 & 0.04 & 0.01 & 0.01 & -0.02 \\
\hline 12 & 6 & -0.03 & 0.01 & -0.10 & -0.01 & -0.07 & 0.01 & 0.00 & 0.01 & -0.01 \\
\hline 13 & 1 & -0.02 & 0.00 & -0.02 & 0.05 & -0.05 & -0.04 & 0.00 & 0.01 & 0.00 \\
\hline 14 & 1 & -0.02 & 0.02 & -0.18 & -0.07 & -0.08 & 0.07 & 0.01 & 0.01 & -0.03 \\
\hline 15 & 1 & -0.09 & 0.04 & -0.11 & 0.01 & -0.10 & 0.01 & 0.00 & 0.02 & -0.01 \\
\hline 16 & 1 & 0.07 & -0.04 & -0.06 & -0.02 & -0.03 & -0.02 & 0.00 & 0.00 & 0.00 \\
\hline 17 & 1 & 0.05 & -0.04 & -0.15 & -0.06 & -0.04 & 0.04 & 0.02 & 0.00 & -0.02 \\
\hline 18 & 8 & 0.01 & -0.01 & -0.15 & 0.02 & -0.10 & 0.01 & 0.00 & 0.01 & 0.00 \\
\hline 19 & 1 & 0.03 & -0.01 & -0.15 & 0.04 & -0.11 & 0.02 & 0.00 & 0.01 & 0.01 \\
\hline 20 & 1 & -0.01 & -0.02 & -0.12 & -0.02 & -0.11 & 0.00 & 0.00 & 0.01 & 0.01 \\
\hline 21 & 7 & 0.01 & -0.02 & -0.07 & 0.03 & -0.08 & -0.03 & -0.01 & 0.00 & 0.00 \\
\hline 22 & 1 & 0.02 & -0.03 & -0.11 & 0.07 & -0.12 & -0.07 & -0.01 & 0.02 & -0.02 \\
\hline 23 & 6 & -0.08 & 0.01 & 0.00 & 0.03 & -0.09 & -0.03 & -0.03 & -0.03 & 0.01 \\
\hline 24 & 1 & -0.06 & 0.01 & 0.06 & 0.07 & -0.06 & -0.06 & -0.04 & -0.04 & -0.01 \\
\hline 25 & 1 & -0.09 & 0.02 & 0.03 & -0.01 & -0.07 & 0.01 & -0.03 & -0.05 & 0.04 \\
\hline 26 & 1 & -0.14 & 0.03 & -0.05 & 0.01 & -0.16 & -0.05 & -0.05 & -0.05 & -0.01 \\
\hline 27 & 6 & 0.10 & -0.04 & -0.01 & 0.05 & 0.02 & -0.01 & 0.02 & 0.02 & 0.02 \\
\hline 28 & 1 & 0.07 & -0.01 & 0.02 & -0.03 & 0.10 & 0.03 & 0.03 & 0.00 & 0.05 \\
\hline 29 & 1 & 0.15 & -0.09 & -0.08 & 0.02 & -0.02 & -0.01 & 0.04 & 0.05 & 0.02 \\
\hline 30 & 6 & 0.18 & -0.04 & 0.08 & 0.19 & 0.09 & -0.04 & -0.01 & 0.02 & -0.01 \\
\hline 31 & 1 & 0.22 & -0.07 & 0.04 & 0.27 & 0.02 & -0.07 & -0.02 & 0.04 & -0.04 \\
\hline 32 & 6 & 0.20 & -0.01 & 0.19 & 0.22 & 0.24 & -0.03 & -0.01 & -0.01 & 0.01 \\
\hline 33 & 1 & 0.16 & 0.02 & 0.23 & 0.14 & 0.31 & 0.00 & 0.00 & -0.04 & 0.04 \\
\hline 34 & 1 & 0.25 & -0.01 & 0.26 & 0.33 & 0.28 & -0.05 & -0.03 & -0.01 & -0.01 \\
\hline
\end{tabular}

thermodynamics:

Sum of electronic and zero-point Energies=

$-1218.713620$

Sum of electronic and thermal Enthalpies=

$-1218.691386$

Sum of electronic and thermal Free Energies=

$-1218.768380$

Total free energy in solution:

with all non electrostatic terms

$(\mathrm{a} \cdot \mathrm{u})=$.

Optimized geometry, three lower frequencies and PCM energy for $\mathbf{T S}_{\mathbf{1 1 c}-\mathbf{1 c}}$.

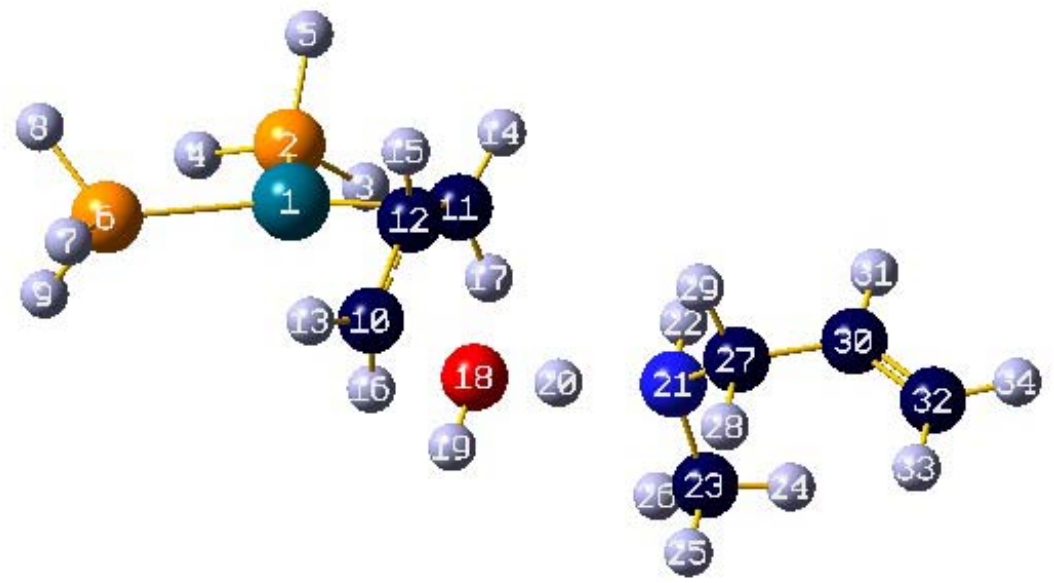

\begin{tabular}{|c|c|c|c|c|c|}
\hline Center & Atomic & Atomic & \multicolumn{3}{|c|}{ Coordinates (Angstroms) } \\
\hline Number & Number & Type & $\mathrm{x}$ & Y & Z \\
\hline 1 & 46 & 0 & 2.096975 & 0.111126 & -0.124972 \\
\hline 2 & 15 & 0 & 3.377419 & 2.031390 & 0.237461 \\
\hline 3 & 1 & 0 & 2.848680 & 3.076337 & 1.026025 \\
\hline 4 & 1 & 0 & 4.663646 & 1.989081 & 0.820469 \\
\hline 5 & 1 & 0 & 3.711084 & 2.797637 & -0.900471 \\
\hline
\end{tabular}




\begin{tabular}{|c|c|c|c|c|c|}
\hline 6 & 15 & 0 & 3.688158 & -1.627407 & 0.014624 \\
\hline 7 & 1 & 0 & 3.375261 & -2.978642 & -0.257463 \\
\hline 8 & 1 & 0 & 4.833667 & -1.541986 & -0.807131 \\
\hline 9 & 1 & 0 & 4.355057 & -1.840141 & 1.241707 \\
\hline 10 & 6 & 0 & -0.145078 & -1.384093 & 0.197402 \\
\hline 11 & 6 & 0 & 0.197099 & 1.014822 & -0.377071 \\
\hline 12 & 6 & 0 & 0.062189 & -0.349175 & -0.753155 \\
\hline 13 & 1 & 0 & 0.113059 & -2.400659 & -0.076853 \\
\hline 14 & 1 & 0 & 0.156161 & 1.782352 & -1.147096 \\
\hline 15 & 1 & 0 & 0.050919 & -0.621975 & -1.806794 \\
\hline 16 & 1 & 0 & -0.096269 & -1.145933 & 1.256186 \\
\hline 17 & 1 & 0 & -0.127810 & 1.339375 & 0.612628 \\
\hline 18 & 8 & 0 & -2.001647 & -1.832616 & 0.174895 \\
\hline 19 & 1 & 0 & -2.196959 & -2.527764 & 0.825060 \\
\hline 20 & 1 & 0 & -2.727364 & -1.021505 & 0.295701 \\
\hline 21 & 7 & 0 & -3.754487 & 0.031883 & 0.341682 \\
\hline 22 & 1 & 0 & -3.312024 & 0.859103 & -0.061879 \\
\hline 23 & 6 & 0 & -4.166596 & 0.342642 & 1.720628 \\
\hline 24 & 1 & 0 & -4.934537 & 1.123162 & 1.761639 \\
\hline 25 & 1 & 0 & -4.570240 & -0.563185 & 2.183962 \\
\hline 26 & 1 & 0 & -3.292986 & 0.666498 & 2.293777 \\
\hline 27 & 6 & 0 & -4.882054 & -0.367958 & -0.541715 \\
\hline 28 & 1 & 0 & -5.346370 & -1.256079 & -0.098216 \\
\hline 29 & 1 & 0 & -4.436928 & -0.675971 & -1.496300 \\
\hline 30 & 6 & 0 & -5.903569 & 0.710470 & -0.761565 \\
\hline 31 & 1 & 0 & -5.545947 & 1.618644 & -1.251834 \\
\hline 32 & 6 & 0 & -7.191877 & 0.610018 & -0.426812 \\
\hline 33 & 1 & 0 & -7.593411 & -0.283477 & 0.048741 \\
\hline 34 & 1 & 0 & -7.897311 & 1.410990 & -0.631621 \\
\hline
\end{tabular}

\begin{tabular}{|c|c|c|c|c|c|c|c|c|c|c|}
\hline & & & 1 & & & 2 & & & 3 & \\
\hline & & & A & & & A & & & A & \\
\hline Frequ & lencies & -- & -340.05 & & & 14.42 & & & $17.13^{\circ}$ & \\
\hline Red. & masses & ; -- & 6.26 & & & 3.31 & & & $3.49^{\circ}$ & \\
\hline Frc & onsts & -- & 0.42 & & & 0.00 & & & 0.00 & \\
\hline IR Ir & ten & -- & 1657.87 & & & 0.11 & & & 1.03 & \\
\hline Atom & AN & X & $Y$ & Z & $\mathrm{X}$ & $\mathrm{Y}$ & Z & $\mathrm{X}$ & Y & Z \\
\hline 1 & 46 & 0.00 & -0.01 & 0.01 & 0.00 & 0.00 & 0.00 & 0.02 & 0.00 & -0.01 \\
\hline 2 & 15 & -0.01 & 0.00 & -0.02 & -0.03 & 0.01 & 0.05 & 0.01 & 0.01 & -0.05 \\
\hline 3 & 1 & -0.04 & -0.05 & 0.02 & -0.05 & -0.01 & 0.06 & -0.01 & 0.05 & -0.13 \\
\hline 4 & 1 & -0.03 & -0.10 & 0.01 & -0.03 & 0.02 & 0.06 & -0.01 & 0.03 & 0.00 \\
\hline 5 & 1 & 0.01 & 0.01 & 0.00 & -0.03 & 0.03 & 0.06 & 0.07 & -0.05 & -0.08 \\
\hline 6 & 15 & 0.02 & 0.00 & 0.01 & 0.01 & 0.01 & 0.07 & 0.00 & 0.00 & 0.16 \\
\hline 7 & 1 & -0.08 & 0.04 & -0.02 & 0.04 & 0.01 & 0.04 & 0.01 & -0.02 & 0.20 \\
\hline 8 & 1 & 0.00 & 0.01 & -0.01 & 0.05 & 0.03 & 0.13 & 0.05 & -0.05 & 0.22 \\
\hline 9 & 1 & 0.01 & -0.03 & 0.01 & -0.05 & 0.01 & 0.10 & -0.07 & 0.05 & 0.21 \\
\hline 10 & 6 & 0.52 & 0.12 & -0.05 & 0.00 & -0.02 & -0.07 & -0.01 & 0.02 & -0.05 \\
\hline 11 & 6 & 0.03 & 0.07 & -0.01 & 0.00 & -0.02 & -0.07 & 0.03 & 0.00 & -0.14 \\
\hline 12 & 6 & -0.02 & 0.08 & -0.06 & 0.03 & -0.02 & -0.07 & 0.04 & -0.02 & -0.09 \\
\hline 13 & 1 & 0.15 & 0.05 & -0.12 & 0.01 & -0.02 & -0.06 & 0.00 & 0.01 & 0.00 \\
\hline 14 & 1 & -0.01 & 0.09 & 0.01 & 0.03 & -0.02 & -0.07 & 0.07 & -0.04 & -0.17 \\
\hline 15 & 1 & -0.02 & 0.08 & -0.06 & 0.07 & -0.02 & -0.07 & 0.09 & -0.06 & -0.07 \\
\hline 16 & 1 & 0.20 & 0.06 & -0.03 & -0.04 & -0.02 & -0.07 & -0.06 & 0.07 & -0.06 \\
\hline 17 & 1 & 0.04 & 0.02 & 0.00 & -0.03 & -0.02 & -0.08 & -0.01 & 0.04 & -0.17 \\
\hline 18 & 8 & -0.18 & -0.26 & 0.02 & 0.00 & -0.02 & -0.14 & -0.01 & 0.04 & -0.13 \\
\hline 19 & 1 & -0.33 & -0.14 & 0.09 & -0.02 & -0.04 & -0.17 & -0.02 & -0.03 & -0.21 \\
\hline 20 & 1 & 0.21 & -0.45 & -0.01 & 0.00 & -0.02 & -0.13 & -0.02 & 0.02 & -0.07 \\
\hline 21 & 7 & -0.08 & 0.10 & 0.02 & 0.02 & 0.00 & -0.11 & -0.03 & 0.00 & 0.04 \\
\hline 22 & 1 & -0.09 & 0.10 & 0.02 & -0.01 & -0.05 & -0.24 & -0.05 & 0.06 & 0.16 \\
\hline 23 & 6 & -0.06 & 0.04 & 0.01 & 0.17 & 0.15 & -0.10 & -0.07 & -0.20 & 0.08 \\
\hline 24 & 1 & -0.08 & 0.02 & 0.11 & 0.19 & 0.16 & -0.10 & -0.09 & -0.22 & 0.17 \\
\hline 25 & 1 & -0.02 & 0.01 & 0.00 & 0.21 & 0.20 & 0.04 & -0.06 & -0.27 & -0.06 \\
\hline 26 & 1 & -0.05 & 0.03 & 0.01 & 0.24 & 0.19 & -0.23 & -0.09 & -0.26 & 0.14 \\
\hline 27 & 6 & -0.02 & 0.04 & 0.02 & -0.08 & -0.06 & 0.05 & -0.01 & 0.09 & -0.03 \\
\hline 28 & 1 & -0.01 & 0.03 & 0.01 & -0.07 & 0.00 & 0.19 & 0.02 & 0.01 & -0.17 \\
\hline 29 & 1 & 0.00 & 0.04 & 0.02 & -0.19 & -0.17 & 0.03 & 0.02 & 0.24 & -0.07 \\
\hline 30 & 6 & -0.04 & 0.01 & -0.02 & -0.08 & -0.05 & 0.04 & -0.04 & 0.08 & 0.10 \\
\hline 31 & 1 & -0.05 & 0.01 & -0.02 & -0.10 & -0.12 & -0.11 & -0.06 & 0.17 & 0.24 \\
\hline
\end{tabular}




$\begin{array}{rrrrrrrrrrr}32 & 6 & -0.04 & 0.01 & 0.00 & -0.04 & 0.03 & 0.20 & -0.04 & -0.01 & 0.06 \\ 33 & 1 & -0.02 & 0.01 & 0.01 & -0.02 & 0.10 & 0.35 & -0.02 & -0.09 & -0.07 \\ 34 & 1 & -0.04 & 0.01 & 0.02 & -0.04 & 0.03 & 0.19 & -0.07 & -0.01 & 0.17\end{array}$

thermodynamics:

Sum of electronic and zero-point Energies=

Sum of electronic and thermal Enthalpies=

$-1218.669026$

Sum of electronic and thermal Free Energies=

$-1218.747542$

Total free energy in solution:

with all non electrostatic terms

$\left(\mathrm{a} \cdot \mathrm{u}_{\mathbf{l}}\right)=-1219.015406$

Optimized geometry, three lower frequencies and PCM energy for 12c.

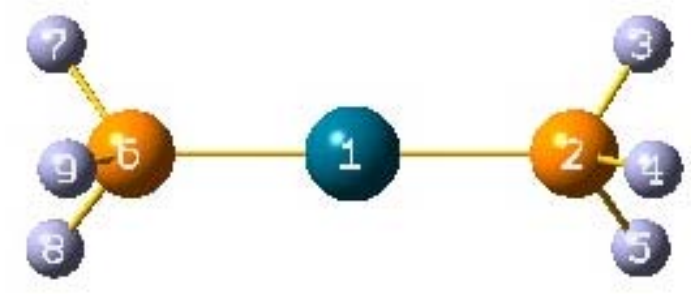

\begin{tabular}{|c|c|c|c|c|c|}
\hline Center & Atomic & Atomic & \multicolumn{3}{|c|}{ Coordinates (Angstroms) } \\
\hline Number & Number & Type & $\mathrm{x}$ & $\mathrm{Y}$ & $\mathrm{Z}$ \\
\hline 1 & 46 & 0 & 0.000007 & 0.000428 & -0.000187 \\
\hline 2 & 15 & 0 & 2.273412 & -0.000420 & 0.000222 \\
\hline 3 & 1 & 0 & 3.006101 & 1.175614 & -0.300091 \\
\hline 4 & 1 & 0 & 3.004093 & -0.329455 & 1.169795 \\
\hline 5 & 1 & 0 & 3.003899 & -0.849811 & -0.868608 \\
\hline 6 & 15 & 0 & -2.273432 & -0.000450 & 0.000287 \\
\hline 7 & 1 & 0 & -3.005356 & 1.173850 & -0.308598 \\
\hline 8 & 1 & 0 & -3.003694 & -0.855673 & -0.863095 \\
\hline 9 & 1 & 0 & -3.005044 & -0.321145 & 1.171553 \\
\hline
\end{tabular}

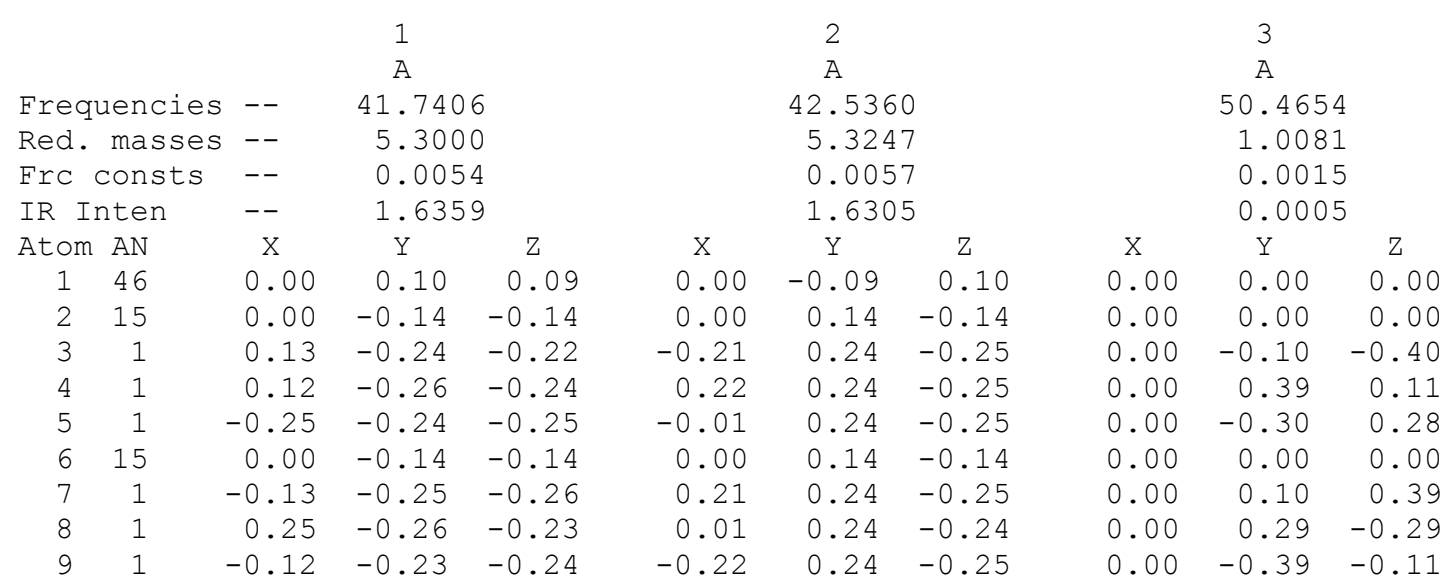

thermodynamics:

Sum of electronic and zero-point Energies=

$-812.980793$

Sum of electronic and thermal Enthalpies=

$-812.972273$

Sum of electronic and thermal Free Energies=

$-813.014577$

Total free energy in solution:

with all non electrostatic terms

$(\mathrm{a} . \mathrm{u})=$. 
Optimized geometry, three lower frequencies and PCM energy for 13c.

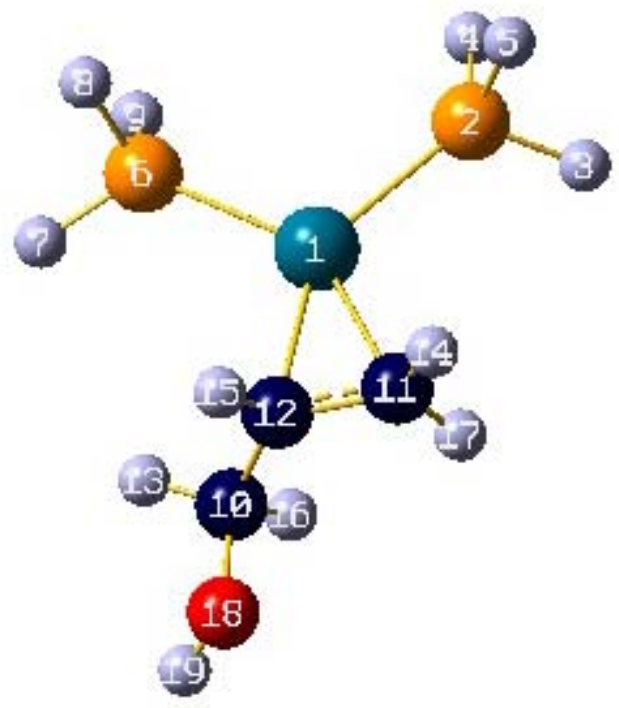

\begin{tabular}{|c|c|c|c|c|c|}
\hline Center & Atomic & Atomic & \multicolumn{3}{|c|}{ Coordinates (Angstroms) } \\
\hline Number & Number & Type & $\mathrm{X}$ & $\mathrm{Y}$ & Z \\
\hline 1 & 46 & 0 & 0.393955 & -0.059550 & 0.101088 \\
\hline 2 & 15 & 0 & 2.395879 & -1.180923 & -0.300710 \\
\hline 3 & 1 & 0 & 2.413746 & -2.400657 & -1.025221 \\
\hline 4 & 1 & 0 & 3.522607 & -0.625556 & -0.965107 \\
\hline 5 & 1 & 0 & 3.132192 & -1.673938 & 0.807163 \\
\hline 6 & 15 & 0 & 0.766452 & 2.233458 & 0.022528 \\
\hline 7 & 1 & 0 & -0.272716 & 3.187646 & -0.142674 \\
\hline 8 & 1 & 0 & 1.341070 & 2.866067 & 1.155070 \\
\hline 9 & 1 & 0 & 1.638491 & 2.838614 & -0.921072 \\
\hline 10 & 6 & 0 & -2.623439 & 0.121224 & -0.520746 \\
\hline 11 & 6 & 0 & -1.057148 & -1.630442 & 0.442457 \\
\hline 12 & 6 & 0 & -1.711605 & -0.397679 & 0.554453 \\
\hline 13 & 1 & 0 & -2.586127 & 1.220070 & -0.561102 \\
\hline 14 & 1 & 0 & -0.768824 & -2.184353 & 1.334350 \\
\hline 15 & 1 & 0 & -1.911708 & -0.000146 & 1.550343 \\
\hline 16 & 1 & 0 & -2.308080 & -0.267776 & -1.500966 \\
\hline 17 & 1 & 0 & -1.172819 & -2.229467 & -0.460838 \\
\hline 18 & 8 & 0 & -3.958397 & -0.306904 & -0.219924 \\
\hline 19 & 1 & 0 & -4.564403 & 0.117392 & -0.844883 \\
\hline
\end{tabular}

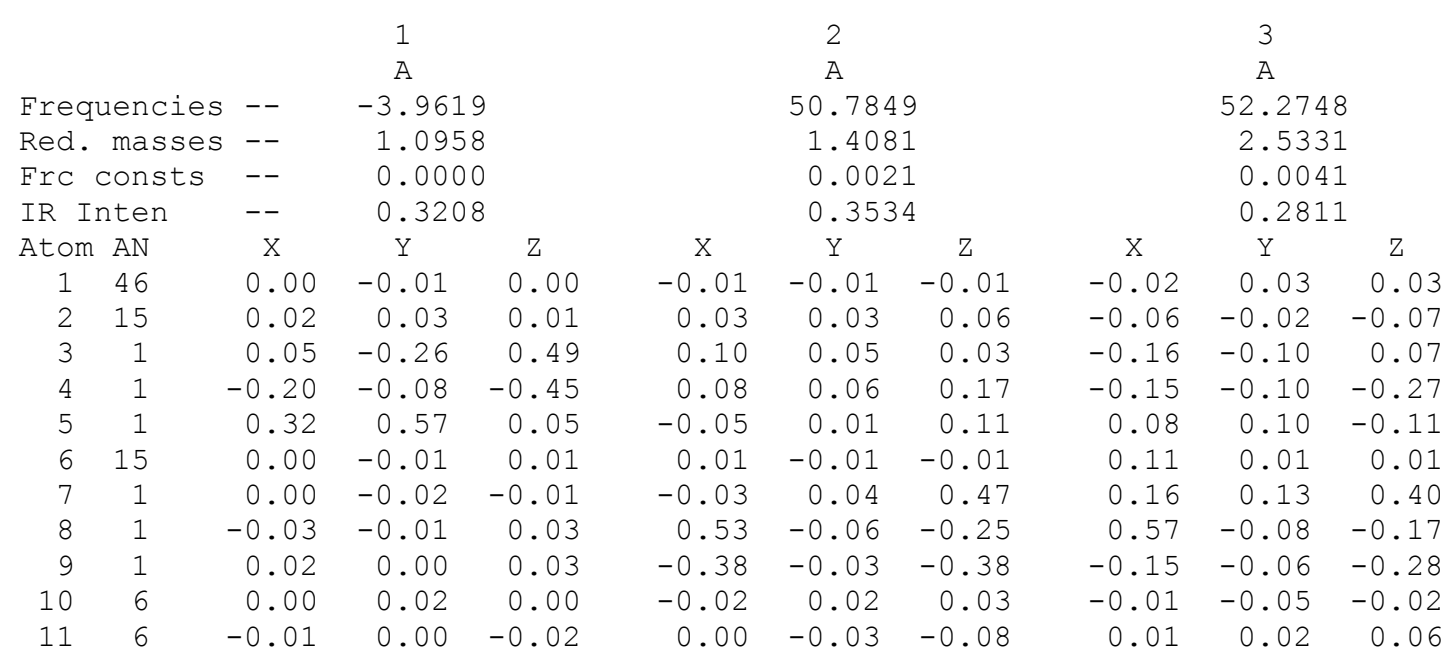




$\begin{array}{rrrrrrrrrrr}12 & 6 & -0.01 & 0.00 & 0.00 & 0.00 & -0.03 & -0.02 & -0.02 & 0.01 & 0.02 \\ 13 & 1 & 0.01 & 0.02 & 0.01 & 0.00 & 0.02 & 0.07 & -0.09 & -0.04 & 0.00 \\ 14 & 1 & -0.01 & -0.01 & -0.02 & 0.00 & -0.08 & -0.11 & 0.01 & 0.05 & 0.08 \\ 15 & 1 & 0.00 & -0.01 & 0.00 & 0.02 & -0.08 & 0.00 & -0.05 & 0.03 & 0.00 \\ 16 & 1 & -0.01 & 0.02 & 0.00 & -0.05 & 0.05 & 0.00 & 0.08 & -0.01 & -0.01 \\ 17 & 1 & -0.02 & 0.01 & -0.02 & -0.01 & 0.02 & -0.11 & 0.02 & 0.00 & 0.08 \\ 18 & 8 & -0.01 & 0.03 & 0.00 & -0.02 & 0.04 & 0.05 & 0.01 & -0.16 & -0.11 \\ 19 & 1 & -0.01 & 0.04 & 0.01 & -0.03 & 0.08 & 0.09 & 0.01 & -0.20 & -0.13\end{array}$

thermodynamics:

Sum of electronic and zero-point Energies=

$-1005.960289$

Sum of electronic and thermal Enthalpies=

$-1005.946752$

Sum of electronic and thermal Free Energies=

$-1006.000997$

Total free energy in solution:

with all non electrostatic terms

$(\mathrm{a} . \mathrm{u})=$.

\section{DDPE-H}

Optimized geometry, three lower frequencies, thermochemistry and PCM energy for $\mathbf{1 d}$.

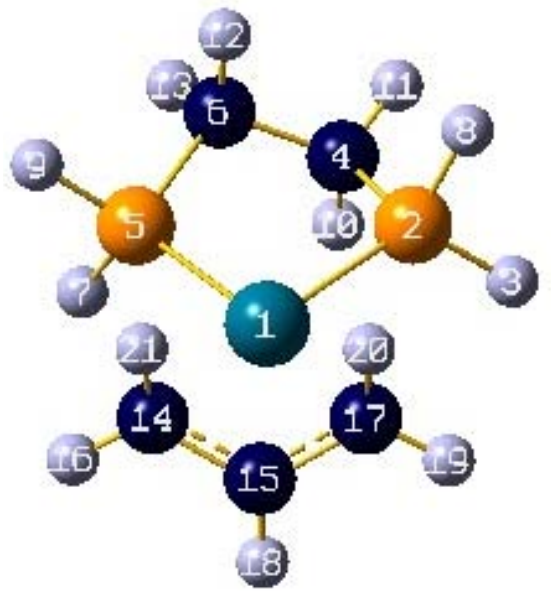

\begin{tabular}{|c|c|c|c|c|c|}
\hline \multirow{2}{*}{$\begin{array}{l}\text { Center } \\
\text { Number }\end{array}$} & \multirow{2}{*}{$\begin{array}{l}\text { Atomic } \\
\text { Number }\end{array}$} & \multirow{2}{*}{$\begin{array}{c}\text { Atomic } \\
\text { Type }\end{array}$} & \multicolumn{3}{|c|}{ Coordinates (Angstroms) } \\
\hline & & & $\mathrm{X}$ & $Y$ & Z \\
\hline 1 & 46 & 0 & 0.578212 & 0.001093 & -0.013421 \\
\hline 2 & 15 & 0 & -1.129365 & -1.585069 & -0.048663 \\
\hline 3 & 1 & 0 & -1.148664 & -2.709158 & 0.800040 \\
\hline 4 & 6 & 0 & -2.717764 & -0.701086 & 0.347301 \\
\hline 5 & 15 & 0 & -1.131377 & 1.583939 & 0.052226 \\
\hline 6 & 6 & 0 & -2.731407 & 0.696147 & -0.284836 \\
\hline 7 & 1 & 0 & -1.353714 & 2.223531 & 1.289420 \\
\hline 8 & 1 & 0 & -1.390573 & -2.214756 & -1.283596 \\
\hline 9 & 1 & 0 & -1.176794 & 2.700654 & -0.805303 \\
\hline 10 & 1 & 0 & -2.778489 & -0.632140 & 1.440348 \\
\hline 11 & 1 & 0 & -3.579813 & -1.287495 & 0.012075 \\
\hline 12 & 1 & 0 & -2.832413 & 0.626484 & -1.374871 \\
\hline 13 & 1 & 0 & -3.582199 & 1.280065 & 0.081889 \\
\hline 14 & 6 & 0 & 2.392152 & 1.221265 & -0.159336 \\
\hline 15 & 6 & 0 & 2.724159 & -0.010407 & 0.439117 \\
\hline 16 & 1 & 0 & 2.487809 & 2.139903 & 0.412529 \\
\hline 17 & 6 & 0 & 2.393379 & -1.211076 & -0.219413 \\
\hline 18 & 1 & 0 & 2.966046 & -0.036914 & 1.500928 \\
\hline 19 & 1 & 0 & 2.489738 & -2.157119 & 0.305755 \\
\hline 20 & 1 & 0 & 2.424780 & -1.267006 & -1.308319 \\
\hline 21 & 1 & 0 & 2.424561 & 1.331568 & -1.243995 \\
\hline
\end{tabular}




\begin{tabular}{|c|c|c|c|c|c|c|c|c|c|c|}
\hline & & & $1_{\mathrm{A}}$ & & & ${ }^{2} \mathrm{~A}$ & & & $\begin{array}{l}3 \\
A\end{array}$ & \\
\hline Frequ & dencies & $5--$ & 60.20 & & & 98.46 & & & 110.444 & \\
\hline Red. & masses & $5--$ & 3.43 & & & 2.59 & & & 4.649 & \\
\hline Frc $c$ & consts & -- & $0.00^{\circ}$ & & & 0.01 & & & 0.033 & \\
\hline IR In & nten & -- & 1.02 & & & 0.10 & & & 5.514 & \\
\hline Atom & AN & X & $\mathrm{Y}$ & Z & X & $\mathrm{Y}$ & Z & $\mathrm{X}$ & Y & Z \\
\hline 1 & 46 & 0.00 & 0.00 & 0.06 & 0.00 & 0.00 & 0.00 & 0.00 & 0.09 & -0.01 \\
\hline 2 & 15 & 0.01 & -0.01 & 0.07 & 0.00 & -0.01 & 0.07 & 0.10 & 0.01 & 0.01 \\
\hline 3 & 1 & -0.07 & 0.08 & 0.19 & 0.04 & 0.07 & 0.18 & 0.22 & 0.04 & 0.05 \\
\hline 4 & 6 & -0.06 & 0.01 & -0.20 & 0.01 & 0.02 & 0.05 & 0.04 & -0.10 & 0.00 \\
\hline 5 & 15 & 0.00 & -0.01 & 0.07 & 0.00 & 0.01 & -0.07 & -0.10 & 0.01 & 0.01 \\
\hline 6 & 6 & 0.05 & 0.00 & -0.20 & 0.01 & -0.02 & -0.05 & -0.04 & -0.11 & -0.01 \\
\hline 7 & 1 & -0.15 & -0.15 & 0.12 & -0.04 & 0.13 & -0.15 & -0.16 & -0.03 & 0.02 \\
\hline 8 & 1 & 0.16 & -0.14 & 0.11 & -0.05 & -0.13 & 0.14 & 0.16 & -0.04 & 0.03 \\
\hline 9 & 1 & 0.07 & 0.08 & 0.19 & 0.05 & -0.08 & -0.19 & -0.21 & 0.03 & 0.03 \\
\hline 10 & 1 & -0.24 & 0.01 & -0.21 & 0.04 & 0.10 & 0.05 & 0.02 & -0.11 & 0.00 \\
\hline 11 & 1 & 0.00 & 0.01 & -0.34 & 0.00 & 0.01 & 0.11 & 0.08 & -0.16 & -0.01 \\
\hline 12 & 1 & 0.24 & 0.01 & -0.22 & 0.03 & -0.10 & -0.04 & -0.02 & -0.12 & -0.01 \\
\hline 13 & 1 & -0.01 & 0.01 & -0.34 & 0.00 & 0.00 & -0.10 & -0.08 & -0.16 & -0.02 \\
\hline 14 & 6 & -0.02 & 0.00 & -0.13 & -0.02 & 0.03 & 0.23 & 0.21 & -0.15 & 0.01 \\
\hline 15 & 6 & 0.05 & 0.00 & -0.17 & 0.00 & -0.08 & 0.00 & -0.01 & -0.20 & 0.01 \\
\hline 16 & 1 & 0.05 & 0.00 & -0.14 & -0.04 & -0.08 & 0.41 & 0.37 & -0.16 & 0.00 \\
\hline 17 & 6 & -0.02 & 0.00 & -0.14 & 0.01 & 0.04 & -0.22 & -0.21 & -0.15 & 0.01 \\
\hline 18 & 1 & 0.16 & 0.00 & -0.20 & 0.00 & -0.28 & -0.01 & -0.02 & -0.24 & 0.01 \\
\hline 19 & 1 & 0.04 & 0.00 & -0.15 & 0.04 & -0.06 & -0.41 & -0.38 & -0.16 & 0.02 \\
\hline 20 & 1 & -0.14 & 0.00 & -0.14 & -0.03 & 0.25 & -0.24 & -0.18 & -0.16 & 0.01 \\
\hline 21 & 1 & -0.13 & 0.01 & -0.13 & 0.03 & 0.24 & 0.25 & 0.21 & -0.16 & 0.01 \\
\hline
\end{tabular}

thermodynamics:

Sum of electronic and zero-point Energies=

$-1007.340336$

Sum of electronic and thermal Enthalpies=

$-1007.328204$

Sum of electronic and thermal Free Energies=

$-1007.377639$

Total free energy in solution:

with all non electrostatic terms

$(\mathrm{a} . \mathrm{u})=$.

\section{Optimized geometry, three lower frequencies, thermochemistry and PCM energy for $\mathbf{T S}_{\mathbf{1 d - 2 d}}$.}

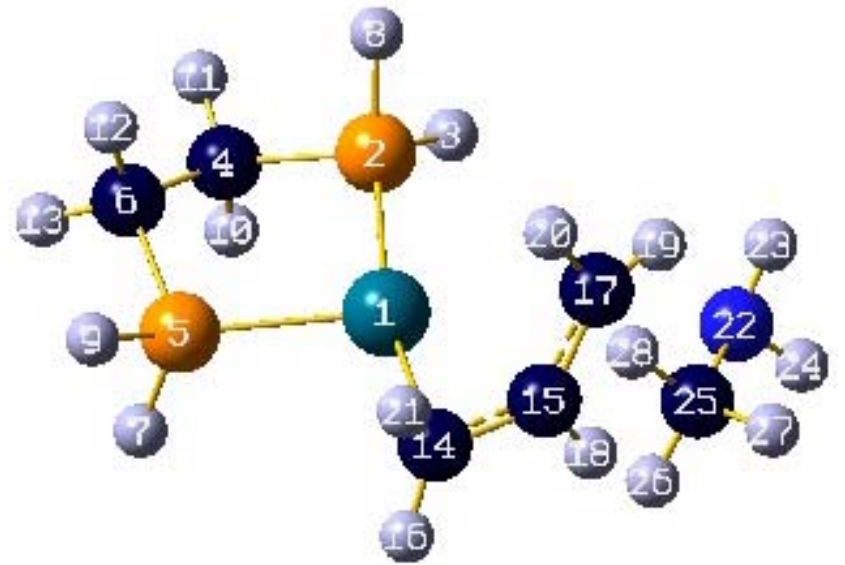

\begin{tabular}{|c|c|c|c|c|c|}
\hline Center & Atomic & Atomic & \multicolumn{3}{|c|}{ Coordinates (Angstroms) } \\
\hline Number & Number & Type & $\mathrm{X}$ & $\mathrm{Y}$ & Z \\
\hline 1 & 46 & 0 & -0.176450 & -0.146235 & -0.175632 \\
\hline 2 & 15 & 0 & -1.645107 & 1.689711 & 0.0071 \\
\hline 3 & 1 & 0 & -1.740778 & 2.785087 & -0.879724 \\
\hline
\end{tabular}




$\begin{array}{rrrrrr}4 & 6 & 0 & -3.372907 & 0.988990 & -0.069746 \\ 5 & 15 & 0 & -2.051829 & -1.470260 & 0.180932 \\ 6 & 6 & 0 & -3.456390 & -0.348891 & 0.675727 \\ 7 & 1 & 0 & -2.580749 & -2.154873 & -0.935635 \\ 8 & 1 & 0 & -1.675741 & 2.415566 & 1.220387 \\ 9 & 1 & 0 & -2.143463 & -2.512906 & 1.127841 \\ 10 & 1 & 0 & -3.609367 & 0.850369 & -1.132082 \\ 11 & 1 & 0 & -4.100420 & 1.701503 & 0.332897 \\ 12 & 1 & 0 & -3.369718 & -0.191021 & 1.757996 \\ 13 & 1 & 0 & -4.422418 & -0.832766 & 0.496389 \\ 14 & 6 & 0 & 1.461490 & -1.469556 & -0.371997 \\ 15 & 6 & 0 & 1.914697 & -0.189682 & -0.803615 \\ 16 & 1 & 0 & 1.340158 & -2.260053 & -1.108681 \\ 17 & 6 & 0 & 2.285291 & 0.836472 & 0.095640 \\ 18 & 1 & 0 & 1.983194 & 0.018242 & -1.870732 \\ 19 & 1 & 0 & 2.277182 & 1.868931 & -0.237268 \\ 20 & 1 & 0 & 2.181157 & 0.680165 & 1.165708 \\ 21 & 1 & 0 & 1.694962 & -1.816840 & 0.635069 \\ 22 & 7 & 0 & 4.373371 & 0.880197 & 0.258863 \\ 23 & 1 & 0 & 4.640348 & 1.578651 & 0.951528 \\ 24 & 1 & 0 & 4.701722 & 1.211681 & -0.646791 \\ 25 & 6 & 0 & 4.933222 & -0.437369 & 0.573974 \\ 26 & 1 & 0 & 4.534279 & -1.169332 & -0.134034 \\ 27 & 1 & 0 & 6.027860 & -0.451938 & 0.519906 \\ 28 & 1 & 0 & 4.626539 & -0.726587 & 1.582647\end{array}$

\begin{tabular}{|c|c|c|c|c|c|c|c|c|c|c|}
\hline & \multicolumn{3}{|c|}{1} & \multicolumn{3}{|c|}{2} & \multicolumn{3}{|c|}{3} \\
\hline & & \multicolumn{3}{|c|}{$\mathrm{A}$} & \multirow{2}{*}{\multicolumn{3}{|c|}{$\begin{array}{c}\text { A } \\
31.8615\end{array}$}} & \multicolumn{3}{|c|}{ A } \\
\hline \multirow{2}{*}{\multicolumn{2}{|c|}{$\begin{array}{l}\text { Frequencies } \\
\text { Red. masses }\end{array}$}} & -- & \multicolumn{2}{|c|}{-243.9666} & & & & & \multicolumn{2}{|c|}{46.4280} \\
\hline & & -- & \multicolumn{2}{|c|}{5.8253} & \multicolumn{3}{|c|}{2.7758} & \multicolumn{3}{|c|}{3.5503} \\
\hline Frc & consts & -- & \multicolumn{2}{|c|}{0.2043} & \multicolumn{3}{|c|}{0.0017} & & \multicolumn{2}{|c|}{0.0045} \\
\hline IR $I_{1}$ & ten & -- & \multicolumn{2}{|c|}{708.9770} & \multicolumn{3}{|c|}{0.1165} & & \multicolumn{2}{|c|}{4.4968} \\
\hline Atom & AN & X & Y & $\mathrm{Z}$ & $\mathrm{X}$ & Y & Z & $x$ & $\mathrm{Y}$ & z \\
\hline 1 & 46 & 0.00 & -0.02 & -0.01 & 0.00 & 0.01 & 0.05 & 0.00 & -0.02 & 0.07 \\
\hline 2 & 15 & 0.01 & 0.00 & -0.02 & -0.03 & 0.00 & -0.04 & 0.04 & 0.00 & 0.09 \\
\hline 3 & 1 & -0.07 & 0.04 & 0.04 & 0.00 & -0.01 & -0.06 & 0.14 & 0.08 & 0.17 \\
\hline 4 & 6 & 0.00 & 0.00 & 0.00 & -0.01 & -0.03 & -0.12 & 0.03 & 0.05 & -0.12 \\
\hline 5 & 15 & -0.01 & 0.00 & 0.02 & 0.01 & 0.00 & 0.01 & -0.05 & 0.01 & -0.08 \\
\hline 6 & 6 & -0.01 & 0.00 & 0.00 & -0.04 & -0.01 & -0.09 & -0.08 & 0.02 & -0.18 \\
\hline 7 & 1 & -0.02 & 0.02 & 0.01 & 0.07 & -0.05 & 0.01 & 0.04 & 0.04 & -0.14 \\
\hline 8 & 1 & -0.01 & -0.03 & 0.00 & -0.10 & 0.01 & -0.05 & -0.04 & -0.10 & 0.14 \\
\hline 9 & 1 & -0.10 & -0.02 & 0.00 & -0.02 & 0.03 & 0.04 & -0.16 & -0.01 & -0.10 \\
\hline 10 & 1 & 0.00 & 0.00 & 0.00 & 0.05 & -0.05 & -0.13 & 0.13 & 0.11 & -0.15 \\
\hline 11 & 1 & 0.00 & 0.01 & 0.00 & -0.05 & -0.03 & -0.18 & 0.01 & 0.05 & -0.16 \\
\hline 12 & 1 & -0.02 & 0.01 & 0.00 & -0.09 & 0.01 & -0.09 & -0.19 & -0.02 & -0.17 \\
\hline 13 & 1 & -0.02 & 0.02 & 0.00 & -0.02 & -0.03 & -0.13 & -0.07 & 0.05 & -0.31 \\
\hline 14 & 6 & 0.04 & 0.08 & 0.01 & 0.00 & 0.02 & 0.07 & 0.00 & -0.03 & 0.03 \\
\hline 15 & 6 & 0.01 & 0.12 & 0.08 & 0.01 & 0.02 & 0.07 & -0.03 & -0.04 & -0.01 \\
\hline 16 & 1 & 0.03 & 0.12 & -0.03 & 0.01 & 0.01 & 0.07 & -0.03 & -0.04 & 0.04 \\
\hline 17 & 6 & 0.51 & 0.01 & 0.12 & 0.01 & 0.01 & 0.07 & 0.01 & -0.02 & -0.04 \\
\hline 18 & 1 & -0.01 & 0.16 & 0.08 & 0.01 & 0.01 & 0.07 & -0.08 & -0.05 & -0.01 \\
\hline 19 & 1 & 0.17 & 0.05 & 0.20 & 0.02 & 0.01 & 0.08 & -0.03 & -0.02 & -0.06 \\
\hline 20 & 1 & 0.22 & -0.01 & 0.09 & 0.01 & 0.00 & 0.07 & 0.08 & 0.00 & -0.03 \\
\hline 21 & 1 & 0.06 & 0.01 & -0.02 & 0.00 & 0.02 & 0.07 & 0.02 & -0.02 & 0.03 \\
\hline 22 & 7 & -0.30 & -0.07 & -0.09 & 0.02 & 0.00 & 0.06 & 0.01 & 0.04 & -0.20 \\
\hline 23 & 1 & -0.40 & 0.00 & -0.11 & 0.01 & -0.17 & 0.23 & 0.05 & 0.18 & -0.36 \\
\hline 24 & 1 & -0.33 & -0.08 & -0.10 & 0.01 & 0.24 & 0.14 & -0.08 & -0.13 & -0.29 \\
\hline 25 & 6 & -0.10 & 0.00 & -0.01 & 0.04 & -0.07 & -0.29 & 0.06 & 0.11 & 0.01 \\
\hline 26 & 1 & 0.01 & -0.07 & 0.00 & 0.02 & 0.10 & -0.45 & 0.03 & -0.03 & 0.17 \\
\hline 27 & 1 & -0.10 & 0.23 & -0.02 & 0.04 & -0.05 & -0.32 & 0.06 & 0.13 & -0.07 \\
\hline 28 & 1 & -0.06 & 0.02 & 0.00 & 0.07 & -0.32 & -0.35 & 0.14 & 0.30 & 0.08 \\
\hline
\end{tabular}

thermodynamics:

Sum of electronic and zero-point Energies=

$-1103.092924$

Sum of electronic and thermal Enthalpies=

$-1103.076842$

Sum of electronic and thermal Free Energies=

$-1103.136712$ 
Total free energy in solution:

with all non electrostatic terms

$\left(\mathrm{a} \cdot \mathrm{u}_{\text {. }}\right)=-1103.379483$

Optimized geometry, three lower frequencies, thermochemistry and PCM energy for $\mathbf{2 d .}$

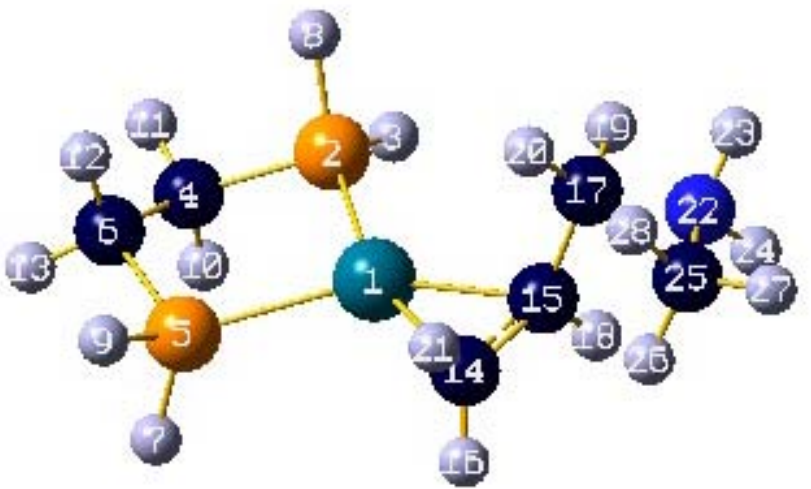

\begin{tabular}{|c|c|c|c|c|c|}
\hline \multirow{2}{*}{$\begin{array}{l}\text { Center } \\
\text { Number }\end{array}$} & \multirow{2}{*}{$\begin{array}{l}\text { Atomic } \\
\text { Number }\end{array}$} & \multirow{2}{*}{$\begin{array}{c}\text { Atomic } \\
\text { Type }\end{array}$} & \multicolumn{3}{|c|}{ Coordinates (Angstroms) } \\
\hline & & & $\mathrm{X}$ & $\mathrm{Y}$ & Z \\
\hline 1 & 46 & 0 & -0.173197 & -0.171728 & -0.184446 \\
\hline 2 & 15 & 0 & -1.632252 & 1.672282 & -0.016437 \\
\hline 3 & 1 & 0 & -1.740027 & 2.770744 & -0.901868 \\
\hline 4 & 6 & 0 & -3.371509 & 0.991731 & -0.093344 \\
\hline 5 & 15 & 0 & -2.078939 & -1.484013 & 0.180088 \\
\hline 6 & 6 & 0 & -3.473906 & -0.338863 & 0.662468 \\
\hline 7 & 1 & 0 & -2.642656 & -2.168548 & -0.922418 \\
\hline 8 & 1 & 0 & -1.685779 & 2.416656 & 1.187835 \\
\hline 9 & 1 & 0 & -2.228383 & -2.517440 & 1.133132 \\
\hline 10 & 1 & 0 & -3.603980 & 0.844664 & -1.155642 \\
\hline 11 & 1 & 0 & -4.093059 & 1.716517 & 0.298207 \\
\hline 12 & 1 & 0 & -3.386768 & -0.170463 & 1.743352 \\
\hline 13 & 1 & 0 & -4.447359 & -0.808984 & 0.486921 \\
\hline 14 & 6 & 0 & 1.573973 & -1.315828 & -0.449130 \\
\hline 15 & 6 & 0 & 1.902920 & 0.057704 & -0.640447 \\
\hline 16 & 1 & 0 & 1.482280 & -1.973913 & -1.311343 \\
\hline 17 & 6 & 0 & 2.621500 & 0.856626 & 0.385574 \\
\hline 18 & 1 & 0 & 1.981270 & 0.441854 & -1.660228 \\
\hline 19 & 1 & 0 & 2.395958 & 1.925679 & 0.332948 \\
\hline 20 & 1 & 0 & 2.455318 & 0.500225 & 1.405795 \\
\hline 21 & 1 & 0 & 1.837857 & -1.822205 & 0.480460 \\
\hline 22 & 7 & 0 & 4.173496 & 0.795561 & 0.193197 \\
\hline 23 & 1 & 0 & 4.614309 & 1.507054 & 0.785348 \\
\hline 24 & 1 & 0 & 4.368431 & 1.067521 & -0.775501 \\
\hline 25 & 6 & 0 & 4.796577 & -0.532587 & 0.462621 \\
\hline 26 & 1 & 0 & 4.315221 & -1.271498 & -0.177979 \\
\hline 27 & 1 & 0 & 5.866197 & -0.478772 & 0.250176 \\
\hline 28 & 1 & 0 & 4.634284 & -0.785274 & 1.511751 \\
\hline
\end{tabular}

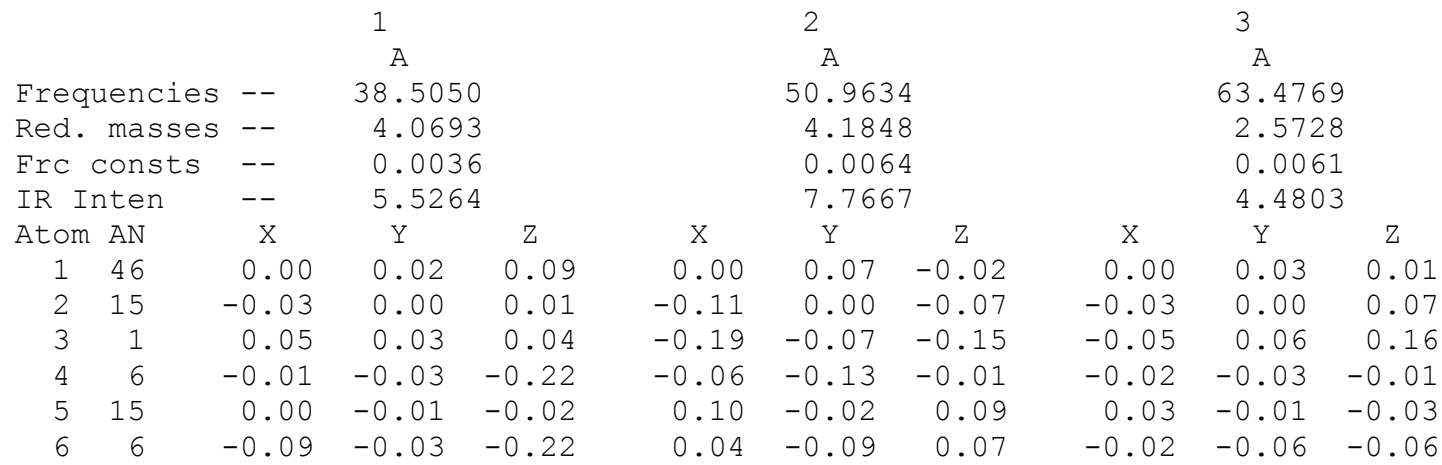




$\begin{array}{rrrrrrrrrrr}7 & 1 & 0.14 & -0.08 & -0.04 & 0.09 & -0.16 & 0.17 & 0.09 & -0.01 & -0.06 \\ 8 & 1 & -0.19 & -0.04 & 0.03 & -0.15 & 0.08 & -0.13 & -0.07 & -0.10 & 0.13 \\ 9 & 1 & -0.07 & 0.03 & 0.02 & 0.23 & 0.04 & 0.18 & 0.03 & -0.03 & -0.05 \\ 10 & 1 & 0.13 & -0.04 & -0.25 & -0.06 & -0.21 & 0.00 & 0.02 & 0.00 & -0.02 \\ 11 & 1 & -0.08 & -0.04 & -0.32 & -0.11 & -0.15 & -0.05 & -0.04 & -0.06 & -0.01 \\ 12 & 1 & -0.23 & -0.01 & -0.21 & 0.06 & -0.02 & 0.06 & -0.08 & -0.09 & -0.05 \\ 13 & 1 & -0.05 & -0.05 & -0.34 & 0.06 & -0.16 & 0.12 & 0.00 & -0.08 & -0.12 \\ 14 & 6 & -0.01 & 0.01 & 0.04 & -0.02 & 0.07 & -0.08 & -0.02 & 0.01 & 0.01 \\ 15 & 6 & -0.01 & 0.01 & 0.04 & 0.02 & 0.07 & 0.00 & 0.00 & -0.01 & -0.07 \\ 16 & 1 & -0.05 & 0.02 & 0.04 & -0.05 & 0.12 & -0.12 & -0.04 & -0.03 & 0.04 \\ 17 & 6 & 0.06 & -0.02 & 0.02 & 0.05 & -0.03 & 0.06 & 0.04 & 0.04 & -0.14 \\ 18 & 1 & -0.04 & 0.02 & 0.04 & 0.05 & 0.13 & 0.02 & -0.03 & -0.06 & -0.09 \\ 19 & 1 & 0.07 & -0.01 & 0.06 & 0.12 & -0.02 & 0.12 & 0.11 & 0.05 & -0.25 \\ 20 & 1 & 0.13 & -0.04 & 0.02 & 0.00 & -0.08 & 0.03 & 0.02 & 0.16 & -0.10 \\ 21 & 1 & 0.02 & 0.00 & 0.03 & -0.03 & 0.01 & -0.11 & -0.02 & 0.05 & 0.03 \\ 22 & 7 & 0.05 & -0.02 & -0.09 & 0.05 & -0.13 & 0.10 & 0.04 & -0.08 & -0.13 \\ 23 & 1 & 0.10 & -0.07 & -0.07 & 0.08 & -0.22 & 0.19 & 0.08 & 0.05 & -0.31 \\ 24 & 1 & -0.02 & 0.05 & -0.09 & 0.10 & -0.05 & 0.13 & 0.05 & -0.33 & -0.20 \\ 25 & 6 & 0.06 & -0.04 & -0.24 & -0.06 & -0.20 & -0.02 & -0.02 & -0.04 & 0.20 \\ 26 & 1 & 0.00 & 0.01 & -0.25 & -0.08 & -0.10 & -0.12 & -0.06 & -0.18 & 0.39 \\ 27 & 1 & 0.04 & -0.04 & -0.31 & -0.04 & -0.25 & 0.04 & -0.02 & -0.15 & 0.19 \\ 28 & 1 & 0.13 & -0.12 & -0.24 & -0.13 & -0.31 & -0.05 & -0.04 & 0.23 & 0.27\end{array}$

thermodynamics:

Sum of electronic and zero-point Energies=

$-1103.100377$

Sum of electronic and thermal Enthalpies=

$-1103.084682$

Sum of electronic and thermal Free Energies=

$-1103.143158$

Total free energy in solution:

with all non electrostatic terms

$($ a.u. $)=-1103.404298$

Optimized geometry, three lower frequencies, thermochemistry and PCM energy for 11d.

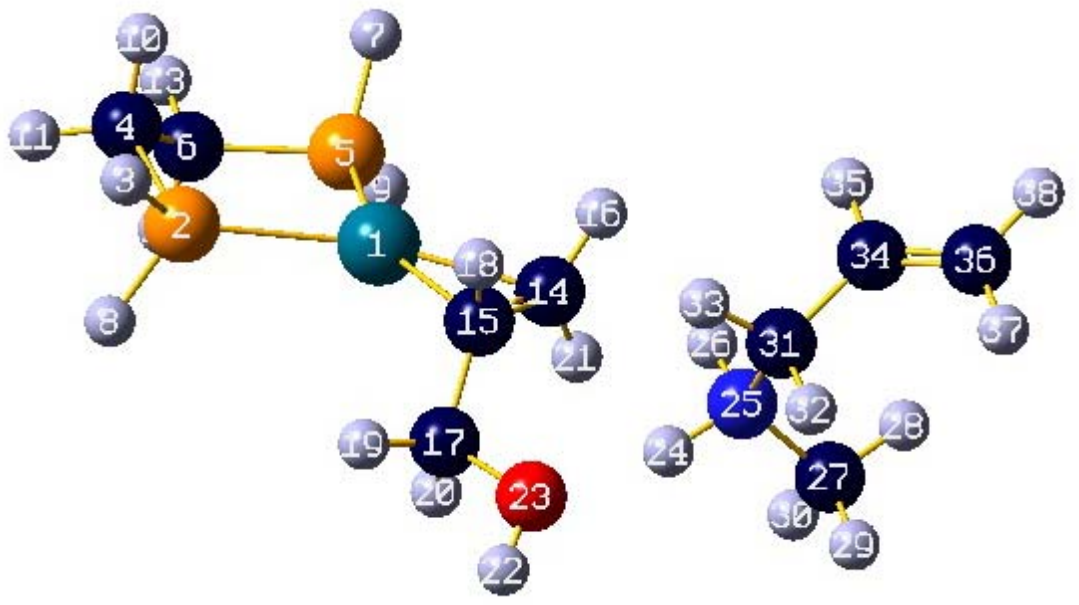

\begin{tabular}{cccrrr}
$\begin{array}{c}\text { Center } \\
\text { Number }\end{array}$ & $\begin{array}{c}\text { Atomic } \\
\text { Number }\end{array}$ & $\begin{array}{c}\text { Atomic } \\
\text { Type }\end{array}$ & \multicolumn{3}{c}{ Coordinates (Angstroms) } \\
-0 & $\mathbf{X}$ & $\mathrm{Y}$ & $\mathrm{Z}$ \\
1 & 46 & 0 & -1.301909 & -0.075111 & 0.001512 \\
2 & 15 & 0 & -3.115565 & 1.155244 & -0.830973 \\
3 & 1 & 0 & -3.243803 & 1.740385 & -2.113116 \\
4 & 6 & 0 & -4.603410 & 0.022276 & -0.818412 \\
5 & 15 & 0 & -2.902053 & -1.674920 & 0.633339 \\
6 & 6 & 0 & -4.585242 & -0.891738 & 0.412838 \\
7 & 1 & 0 & -3.043596 & -2.849935 & -0.144468 \\
8 & 1 & 0 & -3.609025 & 2.257559 & -0.090327 \\
9 & 1 & 0 & -3.058655 & -2.285920 & 1.899957 \\
10 & 1 & 0 & -4.550058 & -0.576113 & -1.736786
\end{tabular}




$\begin{array}{ll}11 & 1 \\ 12 & 1 \\ 13 & 1 \\ 14 & 6 \\ 15 & 6 \\ 16 & 1 \\ 17 & 6 \\ 18 & 1 \\ 19 & 1 \\ 20 & 1 \\ 21 & 1 \\ 22 & 1 \\ 23 & 8 \\ 24 & 1 \\ 25 & 7 \\ 26 & 1 \\ 27 & 6 \\ 28 & 1 \\ 29 & 1 \\ 30 & 1 \\ 31 & 6 \\ 32 & 1 \\ 33 & 1 \\ 34 & 6 \\ 35 & 1 \\ 36 & 6 \\ 37 & 1 \\ 38 & 1\end{array}$

\begin{tabular}{|c|c|c|c|c|c|c|c|c|c|c|}
\hline & & & $1_{A}$ & & & ${ }^{2} A$ & & & ${ }^{3}$ & \\
\hline Frequ & lencies & -- & 17.19 & & & 24.70 & & & 37.88 & \\
\hline Red. & masses & -- & 4.37 & & & 4.33 & & & 2.620 & \\
\hline Frc & onsts & -- & 0.00 & & & 0.00 & & & 0.002 & \\
\hline IR $\operatorname{Ir}$ & ten & -- & 0.60 & & & 0.69 & & & 1.494 & \\
\hline Atom & AN & X & Y & Z & X & $\mathrm{Y}$ & Z & X & Y & Z \\
\hline 1 & 46 & -0.03 & -0.01 & -0.02 & 0.01 & 0.02 & -0.03 & -0.01 & -0.01 & 0.01 \\
\hline 2 & 15 & -0.04 & 0.04 & 0.06 & -0.05 & 0.04 & 0.14 & 0.01 & 0.04 & 0.03 \\
\hline 3 & 1 & -0.08 & 0.05 & 0.07 & -0.13 & 0.14 & 0.19 & 0.03 & 0.08 & 0.05 \\
\hline 4 & 6 & -0.06 & 0.06 & 0.11 & -0.03 & 0.00 & 0.12 & -0.01 & 0.06 & -0.02 \\
\hline 5 & 15 & -0.04 & 0.01 & 0.02 & 0.08 & -0.07 & -0.09 & -0.04 & -0.01 & -0.06 \\
\hline 6 & 6 & -0.03 & 0.05 & 0.09 & 0.05 & -0.10 & 0.04 & -0.03 & 0.01 & -0.05 \\
\hline 7 & 1 & -0.09 & 0.03 & 0.01 & 0.08 & -0.01 & -0.19 & -0.04 & 0.02 & -0.10 \\
\hline 8 & 1 & 0.01 & 0.04 & 0.09 & -0.04 & -0.03 & 0.25 & 0.02 & 0.02 & 0.07 \\
\hline 9 & 1 & 0.00 & 0.00 & 0.02 & 0.15 & -0.18 & -0.14 & -0.07 & -0.06 & -0.08 \\
\hline 10 & 1 & -0.11 & 0.07 & 0.10 & -0.06 & 0.08 & 0.07 & -0.01 & 0.09 & -0.04 \\
\hline 11 & 1 & -0.05 & 0.08 & 0.15 & -0.04 & -0.01 & 0.21 & 0.00 & 0.07 & -0.01 \\
\hline 12 & 1 & 0.02 & 0.04 & 0.11 & 0.08 & -0.17 & 0.10 & -0.03 & -0.01 & -0.04 \\
\hline 13 & 1 & -0.05 & 0.06 & 0.11 & 0.07 & -0.11 & 0.02 & -0.04 & 0.03 & -0.09 \\
\hline 14 & 6 & -0.02 & -0.04 & -0.09 & 0.04 & 0.03 & -0.13 & -0.01 & -0.03 & 0.02 \\
\hline 15 & 6 & -0.02 & -0.04 & -0.08 & -0.01 & 0.07 & -0.06 & -0.01 & -0.02 & 0.03 \\
\hline 16 & 1 & -0.05 & -0.04 & -0.11 & 0.04 & 0.07 & -0.19 & -0.01 & -0.02 & 0.02 \\
\hline 17 & 6 & 0.01 & -0.05 & -0.06 & -0.01 & 0.04 & 0.00 & -0.01 & -0.03 & 0.03 \\
\hline 18 & 1 & -0.05 & -0.03 & -0.08 & -0.05 & 0.13 & -0.07 & -0.01 & -0.02 & 0.03 \\
\hline 19 & 1 & 0.01 & -0.03 & -0.05 & -0.05 & 0.05 & 0.06 & -0.02 & -0.03 & 0.05 \\
\hline 20 & 1 & 0.03 & -0.05 & -0.06 & 0.04 & -0.02 & 0.01 & 0.01 & -0.03 & 0.04 \\
\hline 21 & 1 & 0.01 & -0.06 & -0.10 & 0.06 & -0.03 & -0.13 & -0.02 & -0.04 & 0.02 \\
\hline 22 & 1 & 0.03 & -0.07 & -0.07 & -0.02 & 0.07 & -0.05 & 0.00 & -0.02 & 0.00 \\
\hline 23 & 8 & 0.01 & -0.07 & -0.09 & -0.01 & 0.07 & -0.05 & -0.01 & -0.02 & 0.01 \\
\hline 24 & 1 & -0.03 & -0.08 & -0.05 & 0.02 & 0.08 & -0.07 & -0.01 & -0.01 & -0.07 \\
\hline 25 & 7 & 0.01 & -0.07 & -0.05 & -0.01 & 0.06 & -0.02 & 0.03 & 0.00 & -0.02 \\
\hline 26 & 1 & 0.04 & -0.10 & -0.13 & -0.04 & 0.09 & -0.01 & 0.05 & -0.04 & 0.12 \\
\hline 27 & 6 & -0.08 & -0.07 & 0.01 & -0.06 & 0.08 & 0.02 & 0.15 & 0.18 & -0.05 \\
\hline 28 & 1 & -0.03 & -0.05 & 0.01 & -0.12 & 0.05 & 0.08 & 0.17 & 0.19 & 0.04 \\
\hline 29 & 1 & -0.13 & -0.04 & 0.10 & -0.02 & 0.04 & 0.00 & 0.14 & 0.22 & -0.22 \\
\hline 30 & 1 & -0.15 & -0.14 & -0.05 & -0.08 & 0.17 & 0.00 & 0.23 & 0.28 & 0.01 \\
\hline 31 & 6 & 0.12 & 0.01 & 0.02 & 0.02 & -0.05 & 0.00 & -0.08 & -0.10 & -0.08 \\
\hline 32 & 1 & 0.07 & 0.06 & 0.11 & 0.05 & -0.09 & -0.01 & -0.15 & 0.01 & -0.26 \\
\hline
\end{tabular}




$\begin{array}{rrrrrrrrrrr}33 & 1 & 0.16 & -0.01 & -0.03 & 0.06 & -0.05 & -0.03 & -0.19 & -0.29 & -0.08 \\ 34 & 6 & 0.23 & 0.05 & 0.03 & -0.06 & -0.09 & 0.09 & 0.05 & -0.09 & 0.08 \\ 35 & 1 & 0.30 & 0.00 & -0.06 & -0.10 & -0.05 & 0.09 & 0.12 & -0.21 & 0.27 \\ 36 & 6 & 0.26 & 0.13 & 0.13 & -0.08 & -0.18 & 0.16 & 0.08 & 0.05 & 0.00 \\ 37 & 1 & 0.20 & 0.18 & 0.22 & -0.04 & -0.22 & 0.16 & 0.01 & 0.17 & -0.19 \\ 38 & 1 & 0.35 & 0.16 & 0.13 & -0.13 & -0.21 & 0.22 & 0.18 & 0.05 & 0.12\end{array}$

thermodynamics:

Sum of electronic and zero-point Energies=

$-1296.081575$

Sum of electronic and thermal Enthalpies=

$-1296.059304$

Sum of electronic and thermal Free Energies=

$-1296.135311$

Total free energy in solution:

with all non electrostatic terms

$(\mathrm{a} \cdot \mathrm{u} \cdot)=-1296.451567$

Optimized geometry, three lower frequencies, thermochemistry and PCM energy for $\mathbf{T} \mathbf{S}_{\mathbf{1 1 d}-\mathbf{1 d}}$.

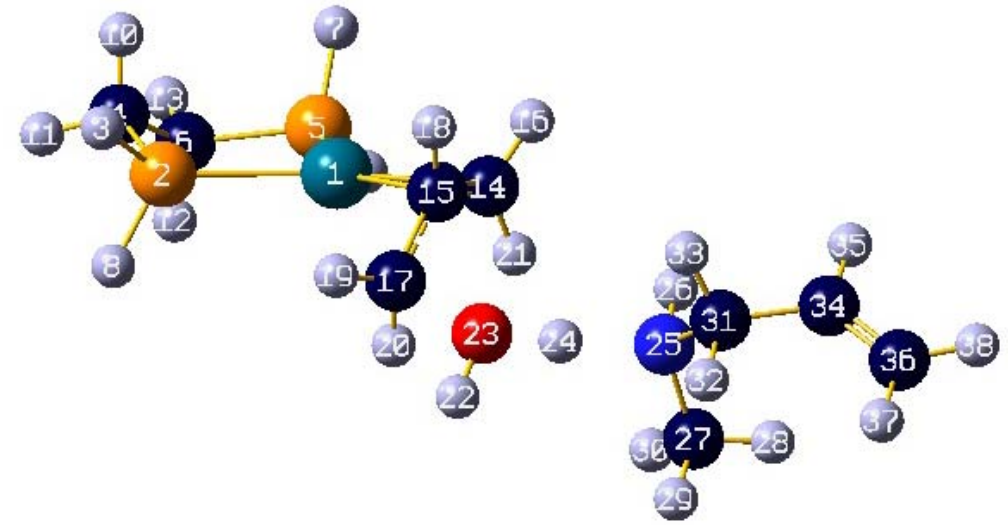

\begin{tabular}{|c|c|c|c|c|c|}
\hline \multirow{2}{*}{$\begin{array}{l}\text { Center } \\
\text { Number }\end{array}$} & \multirow{2}{*}{$\begin{array}{l}\text { Atomic } \\
\text { Number }\end{array}$} & \multirow{2}{*}{$\begin{array}{c}\text { Atomic } \\
\text { Type }\end{array}$} & \multicolumn{3}{|c|}{ Coordinates (Angstroms) } \\
\hline & & & $\mathrm{X}$ & $Y$ & Z \\
\hline 1 & 46 & 0 & -1.549301 & -0.063134 & -0.209982 \\
\hline 2 & 15 & 0 & -3.348962 & 1.444141 & -0.006402 \\
\hline 3 & 1 & 0 & -3.671846 & 2.506436 & -0.880616 \\
\hline 4 & 6 & 0 & -4.911049 & 0.423285 & -0.072561 \\
\hline 5 & 15 & 0 & -3.133744 & -1.737145 & 0.124063 \\
\hline 6 & 6 & 0 & -4.722237 & -0.914837 & 0.652783 \\
\hline 7 & 1 & 0 & -3.543123 & -2.484694 & -1.003554 \\
\hline 8 & 1 & 0 & -3.514812 & 2.143695 & 1.212015 \\
\hline 9 & 1 & 0 & -3.034351 & -2.803547 & 1.044742 \\
\hline 10 & 1 & 0 & -5.133049 & 0.254688 & -1.133790 \\
\hline 11 & 1 & 0 & -5.756491 & 0.976030 & 0.350329 \\
\hline 12 & 1 & 0 & -4.648450 & -0.755189 & 1.735821 \\
\hline 13 & 1 & 0 & -5.580147 & -1.573409 & 0.480361 \\
\hline 14 & 6 & 0 & 0.355817 & -0.952745 & -0.413672 \\
\hline 15 & 6 & 0 & 0.494757 & 0.420118 & -0.766230 \\
\hline 16 & 1 & 0 & 0.420883 & -1.709680 & -1.192200 \\
\hline 17 & 6 & 0 & 0.690823 & 1.448922 & 0.197151 \\
\hline 18 & 1 & 0 & 0.533824 & 0.705328 & -1.816209 \\
\hline 19 & 1 & 0 & 0.427451 & 2.464650 & -0.076047 \\
\hline 20 & 1 & 0 & 0.594893 & 1.202230 & 1.251185 \\
\hline 21 & 1 & 0 & 0.652754 & -1.292055 & 0.580073 \\
\hline 22 & 1 & 0 & 2.701968 & 2.537291 & 0.922922 \\
\hline 23 & 8 & 0 & 2.519459 & 1.874618 & 0.235934 \\
\hline 24 & 1 & 0 & 3.227857 & 1.028726 & 0.344278 \\
\hline 25 & 7 & 0 & 4.192270 & -0.044302 & 0.368492 \\
\hline 26 & 1 & 0 & 3.716699 & -0.836964 & -0.066014 \\
\hline 27 & 6 & 0 & 4.571039 & -0.413917 & 1.742620 \\
\hline 28 & 1 & 0 & 5.302631 & -1.228928 & 1.767928 \\
\hline
\end{tabular}




$\begin{array}{lll}29 & 1 & 0 \\ 30 & 1 & 0 \\ 31 & 6 & 0 \\ 32 & 1 & 0 \\ 33 & 1 & 0 \\ 34 & 6 & 0 \\ 35 & 1 & 0 \\ 36 & 6 & 0 \\ 37 & 1 & 0 \\ 38 & 1 & 0\end{array}$

$$
\begin{array}{lrr}
5.008280 & 0.458798 & 2.237802 \\
3.675766 & -0.715257 & 2.294005 \\
5.347038 & 0.334561 & -0.489125 \\
5.840095 & 1.190164 & -0.014060 \\
4.926873 & 0.687198 & -1.439448 \\
6.326456 & -0.778398 & -0.726488 \\
5.939602 & -1.656335 & -1.248474 \\
7.612304 & -0.740943 & -0.370019 \\
8.042359 & 0.121045 & 0.137649 \\
8.287559 & -1.564103 & -0.588115
\end{array}
$$

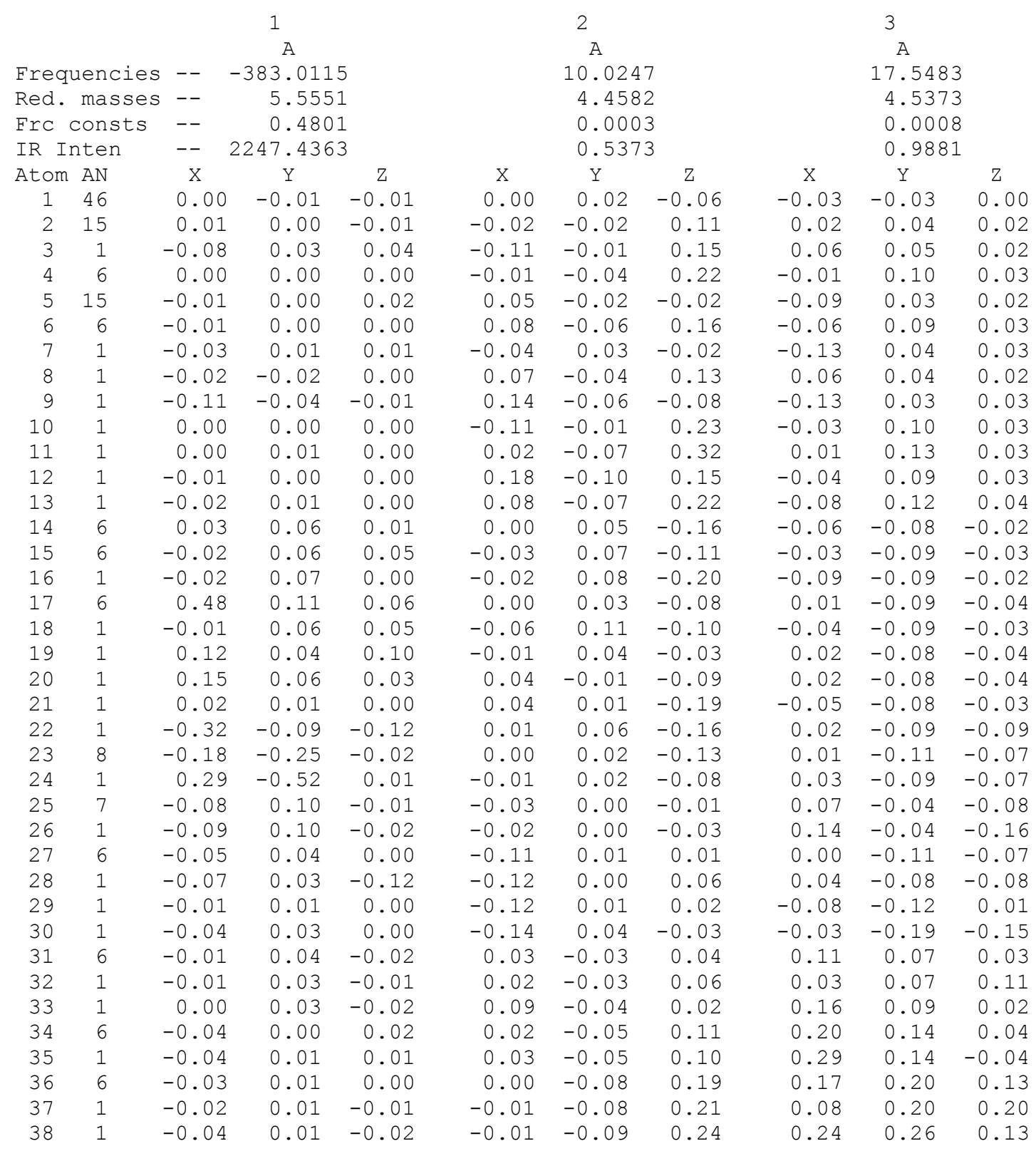

thermodynamics:

Sum of electronic and zero-point Energies=

$-1296.061835$

Sum of electronic and thermal Enthalpies=

$-1296.039702$

Sum of electronic and thermal Free Energies=

$-1296.117064$

Total free energy in solution:

with all non electrostatic terms

$($ a.u. $)=\quad-1296.424027$ 
Optimized geometry, three lower frequencies, thermochemistry and PCM energy for 12d.

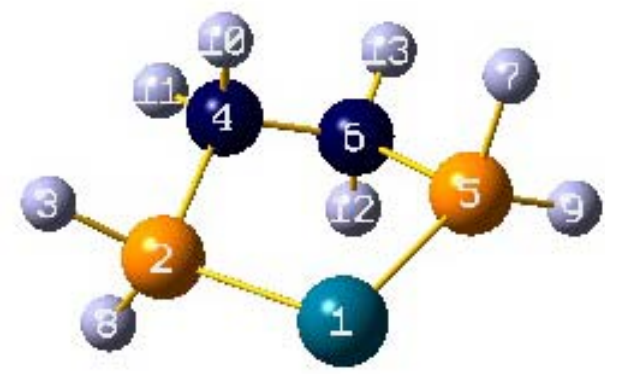

\begin{tabular}{|c|c|c|c|c|c|}
\hline \multirow{2}{*}{$\begin{array}{l}\text { Center } \\
\text { Number }\end{array}$} & \multirow{2}{*}{$\begin{array}{l}\text { Atomic } \\
\text { Number }\end{array}$} & \multirow{2}{*}{$\begin{array}{l}\text { Atomic } \\
\text { Type }\end{array}$} & \multicolumn{3}{|c|}{ Coordinates (Angstroms) } \\
\hline & & & $\mathrm{X}$ & $Y$ & Z \\
\hline 1 & 46 & 0 & 1.082196 & -0.000027 & -0.000023 \\
\hline 2 & 15 & 0 & -0.431816 & -1.693072 & -0.055194 \\
\hline 3 & 1 & 0 & -0.744346 & -2.827801 & 0.749159 \\
\hline 4 & 6 & 0 & -1.986568 & -0.697129 & 0.322521 \\
\hline 5 & 15 & 0 & -0.431690 & 1.693055 & 0.055287 \\
\hline 6 & 6 & 0 & -1.986507 & 0.697311 & -0.322648 \\
\hline 7 & 1 & 0 & -0.840380 & 2.297444 & 1.277520 \\
\hline 8 & 1 & 0 & -0.840688 & -2.297683 & -1.277248 \\
\hline 9 & 1 & 0 & -0.744266 & 2.827943 & -0.748811 \\
\hline 10 & 1 & 0 & -2.028589 & -0.602748 & 1.415575 \\
\hline 11 & 1 & 0 & -2.881618 & -1.250802 & 0.013388 \\
\hline 12 & 1 & 0 & -2.028572 & 0.603016 & -1.415714 \\
\hline 13 & 1 & 0 & -2.881490 & 1.251040 & -0.013442 \\
\hline
\end{tabular}

\begin{tabular}{|c|c|c|c|c|c|c|c|c|c|c|}
\hline & & & 1 & & & 2 & & & 3 & \\
\hline & & & A & & & A & & & A & \\
\hline Frequ & rencies & -- & 106.047 & & & 184.54 & & & 211.430 & \\
\hline Red. & masses & -- & 2.617 & & & 6.15 & & & 1.938 & \\
\hline Frc & onsts & -- & 0.017 & & & 0.12 & & & 0.051 & \\
\hline IR In & ten & -- & 0.408 & & & 0.00 & & & 0.029 & \\
\hline Atom & AN & $\mathrm{x}$ & $\mathrm{Y}$ & Z & $\mathrm{x}$ & $\mathrm{Y}$ & Z & $\mathrm{x}$ & $\mathrm{Y}$ & Z \\
\hline 1 & 46 & 0.00 & 0.00 & 0.05 & 0.13 & 0.00 & 0.00 & 0.02 & 0.00 & 0.00 \\
\hline 2 & 15 & -0.01 & 0.00 & -0.12 & -0.12 & 0.14 & 0.04 & -0.05 & 0.02 & -0.03 \\
\hline 3 & 1 & -0.05 & -0.17 & -0.37 & -0.23 & 0.00 & -0.19 & -0.03 & -0.05 & -0.12 \\
\hline 4 & 6 & 0.05 & -0.01 & 0.13 & -0.23 & -0.01 & -0.04 & 0.05 & 0.07 & 0.15 \\
\hline 5 & 15 & 0.01 & 0.00 & -0.12 & -0.12 & -0.14 & -0.04 & -0.05 & -0.02 & 0.03 \\
\hline 6 & 6 & -0.05 & -0.01 & 0.13 & -0.23 & 0.01 & 0.04 & 0.05 & -0.07 & -0.15 \\
\hline 7 & 1 & 0.07 & 0.30 & -0.25 & 0.04 & -0.32 & 0.10 & -0.21 & -0.17 & 0.06 \\
\hline 8 & 1 & -0.07 & 0.30 & -0.25 & 0.04 & 0.32 & -0.10 & -0.21 & 0.17 & -0.06 \\
\hline 9 & 1 & 0.05 & -0.17 & -0.37 & -0.23 & 0.00 & 0.19 & -0.03 & 0.05 & 0.12 \\
\hline 10 & 1 & 0.22 & -0.03 & 0.14 & -0.36 & -0.08 & -0.04 & 0.27 & 0.30 & 0.14 \\
\hline 11 & 1 & 0.00 & -0.01 & 0.26 & -0.14 & -0.07 & -0.18 & -0.03 & 0.05 & 0.43 \\
\hline 12 & 1 & -0.22 & -0.03 & 0.14 & -0.36 & 0.08 & 0.04 & 0.27 & -0.30 & -0.14 \\
\hline 13 & 1 & 0.00 & -0.01 & 0.26 & -0.14 & 0.07 & 0.18 & -0.03 & -0.05 & -0.43 \\
\hline
\end{tabular}

thermodynamics:

Sum of electronic and zero-point Energies=

$-890.325686$

Sum of electronic and thermal Enthalpies=

$-890.317206$

Sum of electronic and thermal Free Energies=

$-890.358558$

Total free energy in solution:

with all non electrostatic terms $\quad(\mathrm{a} . \mathrm{u} \cdot)=\mathbf{- 8 9 0 . 4 1 7 9 5 9}$

Optimized geometry, three lower frequencies, thermochemistry and PCM energy for 13d. 


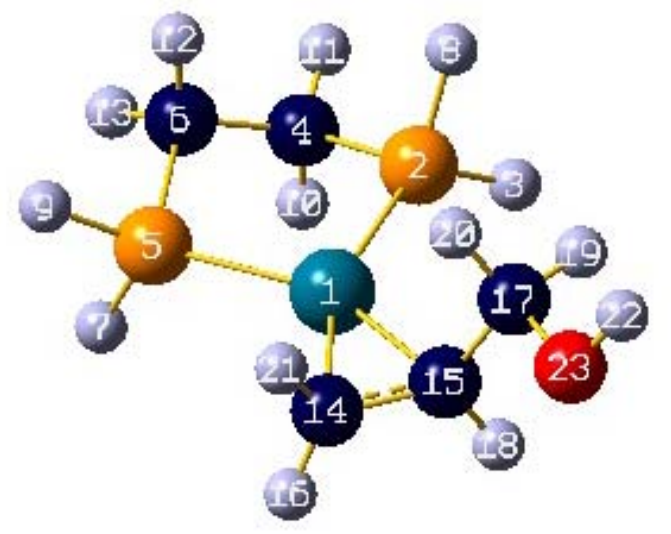

\begin{tabular}{|c|c|c|c|c|c|}
\hline Center & Atomic & Atomic & $\mathrm{COO}$ & inates (An & troms ) \\
\hline Number & Number & Type & $\mathrm{X}$ & $Y$ & Z \\
\hline 1 & 46 & 0 & 0.144643 & -0.272301 & -0.153389 \\
\hline 2 & 15 & 0 & -1.100262 & 1.697577 & -0.130347 \\
\hline 3 & 1 & 0 & -1.137640 & 2.771194 & -1.059254 \\
\hline 4 & 6 & 0 & -2.909467 & 1.199370 & -0.190280 \\
\hline 5 & 15 & 0 & -1.873233 & -1.390244 & 0.201200 \\
\hline 6 & 6 & 0 & -3.154636 & -0.082331 & 0.615238 \\
\hline 7 & 1 & 0 & -2.527774 & -2.029739 & -0.885279 \\
\hline 8 & 1 & 0 & -1.116944 & 2.517972 & 1.029710 \\
\hline 9 & 1 & 0 & -2.228439 & -2.363854 & 1.172000 \\
\hline 10 & 1 & 0 & -3.149962 & 1.029803 & -1.248099 \\
\hline 11 & 1 & 0 & -3.557468 & 2.009362 & 0.163132 \\
\hline 12 & 1 & 0 & -3.053550 & 0.123993 & 1.688855 \\
\hline 13 & 1 & 0 & -4.172341 & -0.454033 & 0.451087 \\
\hline 14 & 6 & 0 & 1.925002 & -1.484876 & -0.277146 \\
\hline 15 & 6 & 0 & 2.284313 & -0.147964 & -0.501618 \\
\hline 16 & 1 & 0 & 1.818196 & -2.176491 & -1.111029 \\
\hline 17 & 6 & 0 & 3.001213 & 0.672437 & 0.532259 \\
\hline 18 & 1 & 0 & 2.436974 & 0.185987 & -1.528946 \\
\hline 19 & 1 & 0 & 2.728750 & 1.733713 & 0.435732 \\
\hline 20 & 1 & 0 & 2.720336 & 0.336542 & 1.542246 \\
\hline 21 & 1 & 0 & 2.123037 & -1.949207 & 0.688955 \\
\hline 22 & 1 & 0 & 4.875686 & 1.124743 & 0.925741 \\
\hline 23 & 8 & 0 & 4.413931 & 0.514508 & 0.332192 \\
\hline
\end{tabular}

\begin{tabular}{|c|c|c|c|c|c|c|c|c|c|c|}
\hline & \multicolumn{3}{|c|}{1} & \multicolumn{3}{|c|}{2} & \multicolumn{3}{|c|}{3} \\
\hline & & \multicolumn{3}{|c|}{ A } & \multicolumn{3}{|c|}{ A } & \multicolumn{3}{|c|}{ A } \\
\hline \multicolumn{2}{|c|}{ Frequencies } & $5--$ & \multicolumn{2}{|c|}{32.4963} & \multicolumn{3}{|c|}{51.7021} & \multicolumn{3}{|c|}{74.3105} \\
\hline \multicolumn{2}{|c|}{ Red. masses } & $5--$ & \multicolumn{2}{|c|}{4.6256} & \multicolumn{3}{|c|}{4.6983} & \multicolumn{3}{|c|}{3.0910} \\
\hline \multicolumn{2}{|c|}{ Frc consts } & -- & \multicolumn{2}{|c|}{0.0029} & \multicolumn{3}{|c|}{0.0074} & & \multicolumn{2}{|c|}{0.0101} \\
\hline IR I & iten & -- & \multicolumn{2}{|c|}{0.4013} & \multicolumn{3}{|c|}{1.1091} & & \multicolumn{2}{|c|}{0.6155} \\
\hline Atom & AN & $\mathrm{X}$ & $\mathrm{Y}$ & Z & $\mathrm{x}$ & $\mathrm{Y}$ & Z & $\mathrm{x}$ & $\mathrm{Y}$ & $\mathrm{z}$ \\
\hline 1 & 46 & 0.00 & 0.02 & 0.10 & 0.00 & 0.08 & -0.01 & 0.00 & -0.04 & 0.01 \\
\hline 2 & 15 & -0.02 & 0.00 & 0.08 & -0.11 & 0.01 & -0.05 & 0.06 & 0.00 & 0.01 \\
\hline 3 & 1 & 0.08 & 0.06 & 0.14 & -0.17 & -0.04 & -0.11 & 0.15 & -0.03 & -0.03 \\
\hline 4 & 6 & 0.00 & -0.03 & -0.20 & -0.07 & -0.12 & -0.06 & 0.06 & 0.02 & -0.10 \\
\hline 5 & 15 & 0.00 & -0.01 & -0.04 & 0.08 & -0.03 & 0.11 & -0.02 & 0.02 & 0.10 \\
\hline 6 & 6 & -0.10 & -0.04 & -0.25 & 0.00 & -0.08 & 0.02 & 0.00 & 0.08 & -0.04 \\
\hline 7 & 1 & 0.16 & -0.07 & -0.10 & 0.08 & -0.21 & 0.22 & -0.05 & -0.12 & 0.19 \\
\hline 8 & 1 & -0.20 & -0.07 & 0.13 & -0.20 & 0.07 & -0.09 & 0.00 & 0.03 & -0.02 \\
\hline 9 & 1 & -0.10 & 0.01 & -0.05 & 0.23 & 0.06 & 0.24 & 0.00 & 0.14 & 0.23 \\
\hline 10 & 1 & 0.17 & -0.03 & -0.24 & -0.03 & -0.22 & -0.06 & 0.14 & -0.04 & -0.11 \\
\hline 11 & 1 & -0.07 & -0.05 & -0.30 & -0.14 & -0.14 & -0.14 & 0.04 & 0.05 & -0.19 \\
\hline 12 & 1 & -0.29 & -0.04 & -0.23 & -0.01 & 0.00 & 0.01 & -0.05 & 0.14 & -0.05 \\
\hline 13 & 1 & -0.07 & -0.06 & -0.42 & 0.02 & -0.15 & 0.04 & -0.01 & 0.09 & -0.06 \\
\hline 14 & 6 & -0.02 & 0.00 & -0.03 & -0.05 & 0.04 & -0.14 & 0.04 & 0.02 & -0.25 \\
\hline 15 & 6 & -0.02 & 0.00 & -0.03 & 0.02 & 0.04 & -0.03 & -0.03 & 0.07 & -0.04 \\
\hline 16 & 1 & -0.08 & 0.02 & -0.03 & -0.09 & 0.12 & -0.20 & 0.03 & 0.15 & -0.36 \\
\hline 17 & 6 & 0.08 & -0.03 & -0.08 & 0.06 & -0.11 & 0.06 & -0.08 & -0.06 & 0.09 \\
\hline
\end{tabular}




$\begin{array}{rrrrrrrrrrr}18 & 1 & -0.09 & 0.02 & -0.04 & 0.03 & 0.13 & 0.00 & -0.06 & 0.25 & 0.01 \\ 19 & 1 & 0.08 & -0.02 & -0.03 & 0.18 & -0.07 & 0.13 & -0.18 & -0.06 & 0.30 \\ 20 & 1 & 0.18 & -0.05 & -0.06 & -0.02 & -0.16 & 0.02 & -0.02 & -0.28 & 0.03 \\ 21 & 1 & 0.04 & -0.01 & -0.05 & -0.04 & -0.06 & -0.19 & 0.09 & -0.13 & -0.33 \\ 22 & 1 & 0.12 & -0.05 & -0.25 & 0.09 & -0.35 & 0.17 & -0.12 & 0.04 & 0.15 \\ 23 & 8 & 0.06 & -0.03 & -0.22 & 0.05 & -0.25 & 0.11 & -0.07 & 0.12 & 0.03\end{array}$

\section{CDA calculations}

Description of the method.

In the CDA method the (canonical, natural, or Kohn Sham) molecular orbitals of the complex are expressed in terms of MOs of appropriately chosen fragments. In the cases studied, the Kohn-Sham orbitals of the calculations are formed in the CDA procedure as a linear combination of the MOs of the methylallylammonium ligand and those of the remaining $\left[\mathrm{PdL}_{2}\right]$ fragment $\left(\mathrm{L}_{2}\right.$ meaning representing two monodentate ligand or a bidentate ligand). In all cases, the ligands and the metal fragments were computed in the geometry of the complex. The orbital contributions are divided in four parts: (i) the mixing of the occupied MOs of the ligand and the unoccupied MOs of the metal fragment. This value (noted d) represents the $\mathrm{L} \rightarrow \mathrm{M}$ donation; (ii) the mixing of the unoccupied MOs of the ligand and the occupied MOs of the metal fragment. This value (noted b) accounts for the $M \rightarrow L$ back donation; (iii) the mixing of the occupied MOs of the ligand and the occupied MOs of the metal fragment. This term (noted r), which describes the repulsive polarization between the ligand and metal fragment, is negative because electronic charge is removed from the overlapping area of the occupied orbitals; (iv) the residual term $(\Delta)$ which results from the mixing of the unoccupied MOs of the two respective fragments. Usually this term is very close to zero for closed-shell interactions. This value constitutes an important probe to determine whether the bonding studied can be really classified as a donor-acceptor interaction following the Dewar-Chatt-Duncansson model. Important deviations from $\Delta=0$ imply that the bond studied is more conventionally described as a normal covalent bond between two open shell fragments. A more detailed presentation of the CDA method and the interpretation of the results can be found in the literature. Compositions of molecular orbitals, overlap 
populations between molecular fragments, bond orders and density-of-states spectra were calculated using the AOMix program developed by I. Gorelski (version 6.23) ( for references see: (a) Gorelsky, S. I. AOMIX: Program for Molecular Orbital Analysis, http://www.sgchem.net/ York University: Toronto, Canada, 1997; (b) Gorelsky, S. I. J. Organomet. Chem. 2001, 635, 187-196).

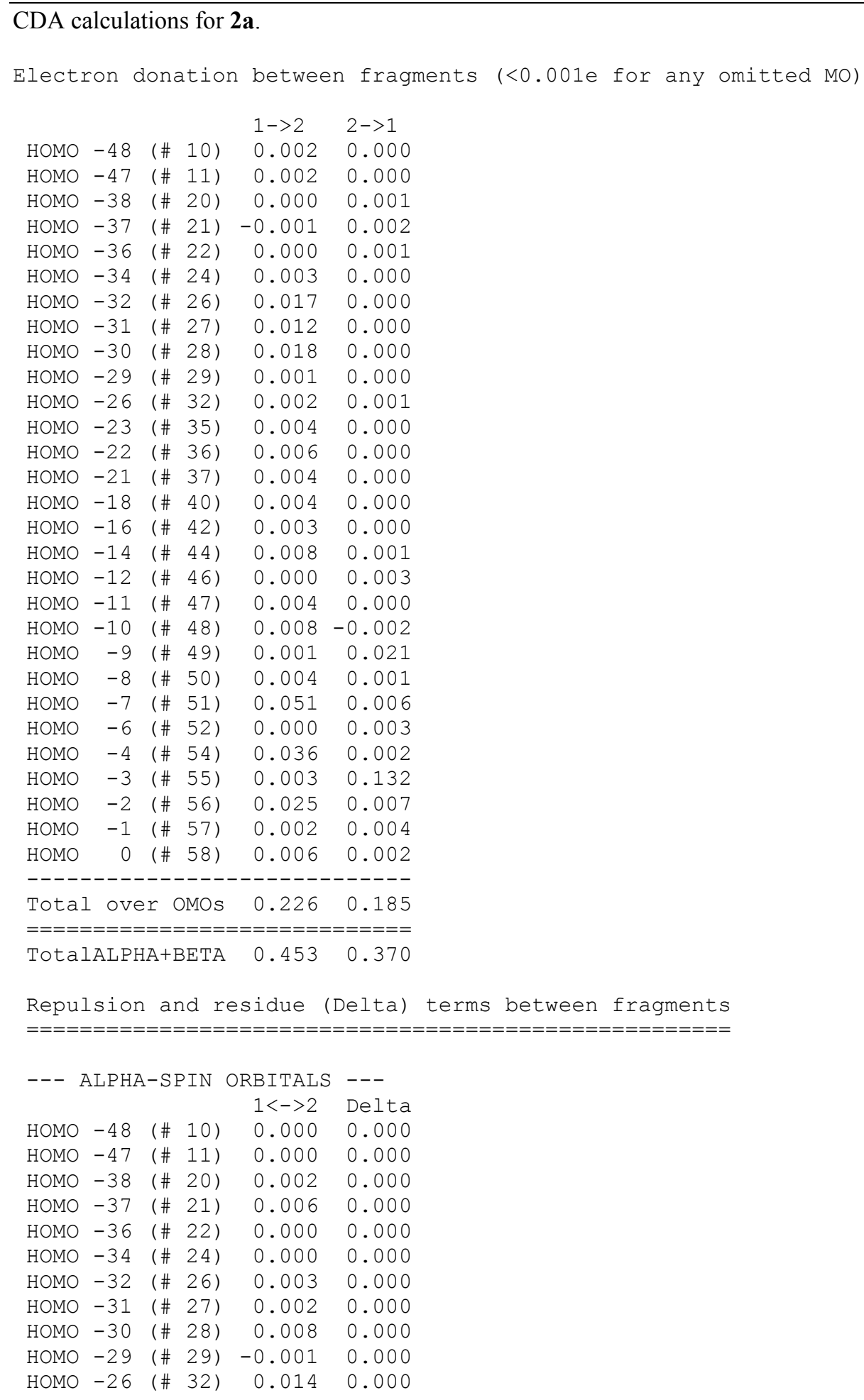




\begin{tabular}{|c|c|c|c|c|c|}
\hline HOMO & -23 & (\# & 35) & 0.008 & 0.000 \\
\hline HOMO & -22 & (\# & 36) & 0.003 & 0.000 \\
\hline HOMO & -21 & (\# & 37) & 0.010 & 0.000 \\
\hline НОМо & -18 & (\# & $40)$ & 0.004 & 0.000 \\
\hline HOMO & -16 & (\# & 42) & 0.014 & 0.000 \\
\hline HOMO & -14 & (\# & 44) & 0.020 & -0.001 \\
\hline HOMO & -12 & (\# & 46) & -0.002 & 0.000 \\
\hline HOMO & -11 & (\# & 47) & 0.011 & 0.000 \\
\hline HOMO & -10 & (\# & 48) & 0.034 & -0.001 \\
\hline HOMO & -9 & (\# & 49) & -0.011 & 0.000 \\
\hline HOMO & -8 & (\# & $50)$ & -0.002 & 0.000 \\
\hline HOMO & -7 & (\# & 51) & -0.057 & -0.003 \\
\hline HOMO & -6 & (\# & 52) & -0.021 & 0.000 \\
\hline HOMO & -4 & (\# & 54) & -0.077 & 0.002 \\
\hline HOMO & -3 & (\# & 55) & -0.042 & -0.001 \\
\hline HOMO & -2 & (\# & 56) & -0.041 & 0.002 \\
\hline HOMO & -1 & (\# & 57) & -0.022 & 0.000 \\
\hline HOMO & 0 & (\# & 58) & -0.016 & 0.000 \\
\hline
\end{tabular}

\section{CDA calculations for $\mathbf{2 b}$.}

Electron donation between fragments $(<0.001$ e for any omitted MO)

\begin{tabular}{|c|c|c|c|c|c|}
\hline & & & & $1->2$ & $2->1$ \\
\hline HOMO & -36 & (\# & 8) & 0.001 & 0.000 \\
\hline HOMO & -35 & (\# & 9) & 0.001 & 0.000 \\
\hline HOMO & -34 & (\# & 10) & 0.000 & 0.001 \\
\hline HOMO & -30 & (\# & 14) & 0.003 & 0.000 \\
\hline HOMO & -27 & (\# & 17) & 0.021 & 0.000 \\
\hline HOMO & -26 & (\# & 18) & 0.015 & 0.000 \\
\hline HOMO & -25 & (\# & 19) & 0.024 & 0.000 \\
\hline HOMO & -24 & (\# & 20) & 0.001 & 0.000 \\
\hline HOMO & -22 & (\# & 22) & 0.001 & 0.001 \\
\hline HOMO & -20 & (\# & 24) & -0.001 & 0.000 \\
\hline HOMO & -19 & (\# & 25) & 0.003 & 0.000 \\
\hline HOMO & -18 & (\# & 26) & 0.005 & 0.000 \\
\hline HOMO & -16 & (\# & 28) & 0.004 & 0.000 \\
\hline HOMO & -14 & (\# & 30) & 0.001 & 0.000 \\
\hline HOMO & -13 & (\# & 31) & 0.003 & 0.000 \\
\hline HOMO & -12 & (\# & 32) & 0.006 & 0.001 \\
\hline HOMO & -11 & (\# & 33) & 0.007 & 0.001 \\
\hline HOMO & -10 & (\# & $34)$ & 0.002 & 0.000 \\
\hline HOMO & -9 & (\# & 35) & 0.001 & 0.014 \\
\hline HOMO & -8 & (\# & 36) & 0.003 & 0.002 \\
\hline HOMO & -7 & (\# & 37) & 0.001 & -0.001 \\
\hline HOMO & -5 & (\# & 39) & 0.081 & 0.006 \\
\hline HOMO & -4 & (\# & $40)$ & -0.001 & 0.015 \\
\hline HOMO & -3 & (\# & 41) & 0.014 & 0.091 \\
\hline HOMO & -2 & (\# & 42) & 0.015 & 0.089 \\
\hline HOMO & -1 & (\# & 43) & 0.001 & 0.005 \\
\hline HOMO & 0 & (\# & 44) & 0.034 & 0.008 \\
\hline tal & ov & $r 0$ & DMOS & 0.248 & 0.234 \\
\hline & $T$ & & & 0.496 & \\
\hline
\end{tabular}

Repulsion and residue (Delta) terms between fragments

\begin{tabular}{|c|c|c|c|c|c|}
\hline$---z$ & $\mathrm{ALPH}$ & & IN & $\begin{array}{c}\text { RBITALS } \\
1<->2\end{array}$ & $\begin{array}{l}--- \\
\text { Delta }\end{array}$ \\
\hline HOMO & -36 & (\# & 8) & 0.000 & 0.000 \\
\hline HOM & -35 & (\# & 9) & 0.000 & 0.000 \\
\hline $\mathrm{HOM}$ & -34 & (\# & 10) & 0.003 & 0.000 \\
\hline HOMO & -30 & (\# & 14) & 0.000 & 0.000 \\
\hline
\end{tabular}




\begin{tabular}{|c|c|c|c|c|c|}
\hline HOMO & -27 & (\# & 17) & 0.002 & 0.000 \\
\hline HOMO & -26 & (\# & 18) & 0.002 & 0.000 \\
\hline HOMO & -25 & (\# & 19) & 0.009 & 0.000 \\
\hline HOMO & -24 & (\# & 20) & -0.001 & 0.000 \\
\hline HOMO & -22 & (\# & 22) & 0.018 & 0.000 \\
\hline HOMO & -20 & (\# & 24) & -0.001 & 0.000 \\
\hline HOMO & -19 & (\# & 25) & 0.009 & 0.000 \\
\hline HOMO & -18 & (\# & 26) & 0.003 & 0.000 \\
\hline HOMO & -16 & (\# & 28) & 0.009 & 0.000 \\
\hline HOMO & -14 & (\# & $30)$ & 0.002 & 0.000 \\
\hline HOMO & -13 & (\# & 31) & -0.001 & 0.000 \\
\hline HOMO & -12 & (\# & 32) & 0.017 & 0.000 \\
\hline HOMO & -11 & (\# & 33) & 0.020 & -0.001 \\
\hline HOMO & -10 & (\# & 34) & 0.004 & 0.000 \\
\hline HOMO & -9 & (\# & $35)$ & -0.003 & -0.001 \\
\hline HOMO & -8 & (\# & 36) & 0.008 & 0.000 \\
\hline HOMO & -7 & (\# & 37) & 0.008 & 0.000 \\
\hline HOMO & -5 & (\# & 39) & -0.058 & -0.005 \\
\hline HOMO & -4 & (\# & $40)$ & -0.045 & 0.000 \\
\hline HOMO & -3 & (\# & 41) & -0.050 & -0.007 \\
\hline HOMO & -2 & (\# & 42) & -0.051 & -0.006 \\
\hline HOMO & -1 & (\# & 43) & -0.019 & 0.000 \\
\hline HOMO & 0 & (\# & $44)$ & -0.054 & 0.002 \\
\hline
\end{tabular}

\section{CDA calculations for $\mathbf{2 c}$.}

Electron donation between fragments $(<0.001$ e for any omitted MO)

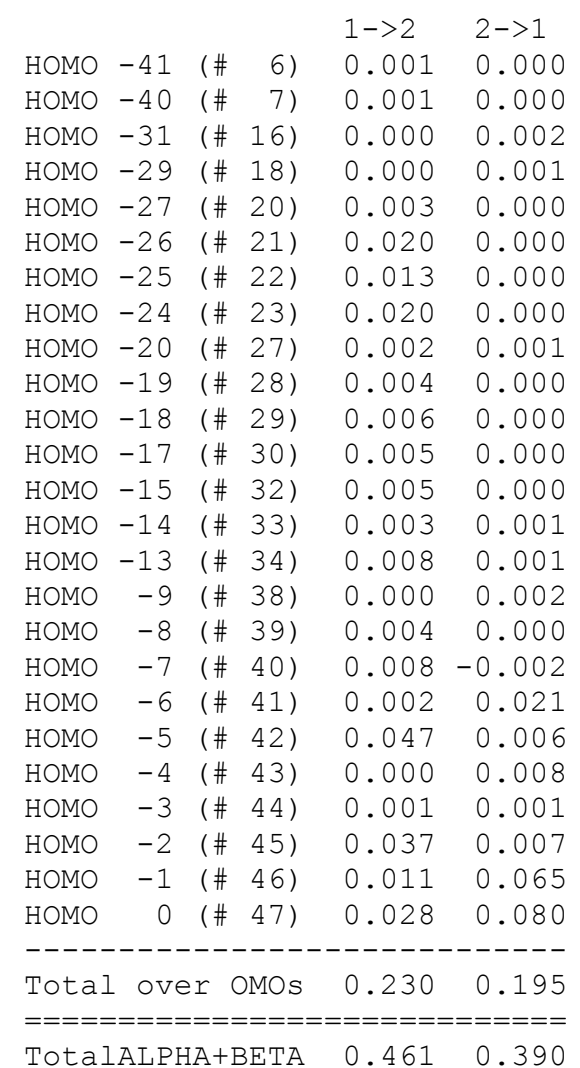

Repulsion and residue (Delta) terms between fragments

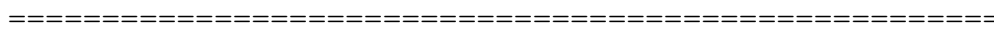

ALPHA-SPIN ORBITALS ---

$1<->2$ Delta

HOMO $-41\left(\begin{array}{llll}\# \quad 6 & 0.000 & 0.000\end{array}\right.$ 


\begin{tabular}{|c|c|c|c|c|c|}
\hline HOMO & -40 & (\# & 7) & 0.000 & 0.000 \\
\hline HOMO & -31 & (\# & 16) & 0.002 & 0.000 \\
\hline HOMO & -29 & (\# & 18) & 0.000 & 0.000 \\
\hline HOMO & -27 & (\# & 20) & 0.000 & 0.000 \\
\hline HOMO & -26 & (\# & 21) & 0.003 & 0.000 \\
\hline HOMO & -25 & (\# & 22) & 0.002 & 0.000 \\
\hline HOMO & -24 & (\# & 23) & 0.008 & 0.000 \\
\hline $\mathrm{HOMO}$ & -20 & (\# & 27) & 0.014 & 0.000 \\
\hline $\mathrm{HOMO}$ & -19 & (\# & 28) & 0.008 & 0.000 \\
\hline HOMO & -18 & (\# & 29) & 0.003 & 0.000 \\
\hline $\mathrm{HOMO}$ & -17 & (\# & $30)$ & 0.010 & 0.000 \\
\hline $\mathrm{HOMO}$ & -15 & (\# & 32) & 0.004 & 0.000 \\
\hline HOMO & -14 & (\# & 33) & 0.014 & 0.000 \\
\hline $\mathrm{HOMO}$ & -13 & (\# & $34)$ & 0.024 & -0.001 \\
\hline $\mathrm{HOMO}$ & -9 & (\# & $38)$ & -0.001 & 0.000 \\
\hline $\mathrm{HOMO}$ & -8 & (\# & 39) & 0.008 & 0.000 \\
\hline HOMO & -7 & (\# & $40)$ & 0.042 & -0.001 \\
\hline $\mathrm{HOMO}$ & -6 & (\# & 41) & -0.011 & -0.001 \\
\hline $\mathrm{HOMO}$ & -5 & (\# & 42) & -0.055 & -0.003 \\
\hline HOMO & -4 & (\# & $43)$ & -0.037 & 0.000 \\
\hline HOMO & -3 & (\# & $44)$ & -0.021 & 0.000 \\
\hline HOMO & -2 & (\# & $45)$ & -0.086 & 0.001 \\
\hline HOMO & -1 & (\# & $46)$ & -0.032 & 0.001 \\
\hline HOMO & 0 & (\# & $47)$ & -0.072 & 0.001 \\
\hline
\end{tabular}

\section{CDA calculations for $\mathbf{2 d}$.}

Electron donation between fragments $(<0.001$ e for any omitted MO)

\begin{tabular}{|c|c|c|c|c|}
\hline & & & $1->2$ & $2->1$ \\
\hline HOMO & -48 & (\# & 0.002 & 0.000 \\
\hline HOMO & -45 & (\# & 0.002 & 0.000 \\
\hline HOMO & -36 & $\left(\begin{array}{ll}\# \text { 18) }\end{array}\right.$ & 0.000 & 0.001 \\
\hline HOMO & -35 & $\left(\begin{array}{ll}\# & 19\end{array}\right)$ & -0.001 & 0.002 \\
\hline HOMO & -34 & $\left(\begin{array}{l}\# \text { 20) } \\
\text { (1) }\end{array}\right.$ & 0.000 & 0.001 \\
\hline HOMO & -32 & $\left(\begin{array}{ll}\# & 22\end{array}\right)$ & 0.002 & 0.000 \\
\hline HOMO & -31 & $\left(\begin{array}{l}\# \text { 23) } \\
\text { (3) }\end{array}\right.$ & 0.023 & 0.000 \\
\hline HOMO & -29 & (\# 25) & 0.012 & 0.000 \\
\hline HOMO & -28 & (\# 26) & 0.022 & 0.000 \\
\hline HOMO & -26 & (\# 28) & 0.002 & 0.000 \\
\hline HOMO & -24 & (\# 30) & 0.003 & 0.001 \\
\hline HOMO & -22 & (\# 32) & 0.003 & 0.000 \\
\hline HOMO & -21 & (\# 33) & 0.006 & 0.000 \\
\hline HOMO & -20 & (\# 34) & 0.003 & 0.000 \\
\hline HOMO & -16 & (\# 38) & 0.002 & 0.000 \\
\hline HOMO & -15 & (\# 39) & 0.006 & 0.000 \\
\hline HOMO & -14 & (\# 40 ) & 0.005 & 0.000 \\
\hline HOMO & -13 & (\# 41) & 0.007 & 0.001 \\
\hline HOMO & -11 & (\# 43) & 0.004 & 0.001 \\
\hline HOMO & -9 & (\# 45) & 0.001 & 0.013 \\
\hline HOMO & -8 & $\left(\begin{array}{ll}\# & 46\end{array}\right)$ & 0.008 & -0.003 \\
\hline HOMO & -6 & $\left(\begin{array}{ll}\# & 48\end{array}\right)$ & 0.001 & 0.017 \\
\hline HOMO & -5 & $\left(\begin{array}{ll}\# & 49\end{array}\right)$ & 0.048 & 0.007 \\
\hline HOMO & -4 & (\# & 0.000 & 0.006 \\
\hline HOMO & -3 & $\left(\begin{array}{l}\# \quad 51\end{array}\right)$ & 0.005 & 0.002 \\
\hline HOMO & -2 & $\left(\begin{array}{ll}\# \quad 52\end{array}\right)$ & 0.036 & 0.001 \\
\hline HOMO & -1 & $\left(\begin{array}{ll}\# & 53\end{array}\right)$ & 0.032 & 0.006 \\
\hline HOMO & 0 & 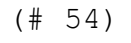 & 0.005 & 0.142 \\
\hline----- & ---- & ------- & ------- & ------- \\
\hline $\begin{array}{l}\text { Total } \\
=====\end{array}$ & $\begin{array}{l}1 \text { ove } \\
=====\end{array}$ & $\begin{array}{l}\text { I OMOS } \\
=======\end{array}$ & $\begin{array}{r}0.237 \\
=======\end{array}$ & $\begin{aligned} & 0.200 \\
== & ====\end{aligned}$ \\
\hline Total & $1 \mathrm{ALPH}$ & $\mathrm{HA}+\mathrm{BETA}$ & 0.474 & 0.401 \\
\hline
\end{tabular}




\begin{tabular}{|c|c|c|c|c|c|}
\hline & & & & $1<->2$ & Delta \\
\hline HOMO & -48 & (\# & 6) & 0.000 & 0.000 \\
\hline HOMO & -45 & (\# & 9) & 0.000 & 0.000 \\
\hline HOMO & -36 & (\# & 18) & 0.002 & 0.000 \\
\hline HOMO & -35 & (\# & 19) & 0.006 & 0.000 \\
\hline HOMO & -34 & (\# & 20) & 0.000 & 0.000 \\
\hline HOMO & -32 & (\# & 22) & 0.000 & 0.000 \\
\hline HOMO & -31 & (\# & 23) & 0.003 & 0.000 \\
\hline HOMO & -29 & (\# & 25) & 0.002 & 0.000 \\
\hline HOMO & -28 & (\# & 26) & 0.008 & 0.000 \\
\hline HOMO & -26 & (\# & 28) & 0.002 & 0.000 \\
\hline HOMO & -24 & (\# & $30)$ & 0.015 & 0.000 \\
\hline HOMO & -22 & (\# & 32) & 0.004 & 0.000 \\
\hline HOMO & -21 & (\# & 33) & 0.005 & 0.000 \\
\hline HOMO & -20 & (\# & 34) & 0.010 & 0.000 \\
\hline HOMO & -16 & (\# & 38) & 0.004 & 0.000 \\
\hline HOMO & -15 & (\# & 39) & 0.009 & 0.000 \\
\hline HOMO & -14 & (\# & $40)$ & 0.010 & 0.000 \\
\hline HOMO & -13 & (\# & 41) & 0.013 & -0.001 \\
\hline HOMO & -11 & (\# & 43) & 0.014 & 0.000 \\
\hline HOMO & -9 & (\# & 45) & -0.010 & 0.000 \\
\hline HOMO & -8 & (\# & 46) & 0.033 & -0.001 \\
\hline HOMO & -6 & （\# & 48) & -0.008 & 0.000 \\
\hline HOMO & -5 & (\# & 49) & -0.066 & -0.003 \\
\hline HOMO & -4 & (\# & $50)$ & -0.042 & 0.000 \\
\hline HOMO & -3 & (\# & 51) & -0.023 & 0.000 \\
\hline HOMO & -2 & (\# & 52) & -0.075 & 0.001 \\
\hline HOMO & -1 & (\# & $53)$ & -0.043 & 0.001 \\
\hline HOMO & 0 & (\# & $54)$ & -0.042 & -0.002 \\
\hline
\end{tabular}

\section{CDA calculations for $\mathbf{1 5 a}$.}

Electron donation between fragments $(<0.001$ e for any omitted MO)

\begin{tabular}{|c|c|c|c|c|c|}
\hline & & & & $1->2$ & $2->1$ \\
\hline HOMO & -48 & (\# & 10) & 0.002 & 0.000 \\
\hline HOMO & -47 & (\# & 11) & 0.002 & 0.000 \\
\hline HOMO & -38 & (\# & 20) & -0.001 & 0.002 \\
\hline HOMO & -34 & (\# & 24) & 0.003 & 0.000 \\
\hline HОMO & -32 & (\# & 26) & 0.010 & 0.000 \\
\hline HOMO & -31 & (\# & 27) & 0.011 & 0.000 \\
\hline HOMO & -30 & (\# & 28) & 0.006 & 0.000 \\
\hline HOMO & -29 & (\# & 29) & 0.006 & 0.000 \\
\hline HOMO & -26 & (\# & 32) & 0.001 & 0.001 \\
\hline НОМО & -25 & (\# & 33) & -0.005 & 0.001 \\
\hline HOMO & -24 & (\# & 34) & -0.001 & -0.001 \\
\hline HОMO & -23 & (\# & 35) & 0.002 & 0.001 \\
\hline HOMO & -22 & (\# & 36) & 0.006 & 0.000 \\
\hline HOMO & -21 & (\# & 37) & 0.001 & 0.001 \\
\hline НОМО & -20 & (\# & 38) & 0.002 & 0.000 \\
\hline HOMO & -18 & (\# & $40)$ & 0.004 & 0.000 \\
\hline НОМО & -17 & (\# & 41) & 0.002 & 0.000 \\
\hline HOMO & -16 & (\# & 42) & -0.003 & -0.001 \\
\hline HOMO & -14 & (\# & 44) & 0.008 & 0.001 \\
\hline НОМО & -12 & (\# & 46) & 0.000 & 0.012 \\
\hline HOMO & -11 & (\# & 47) & -0.002 & -0.005 \\
\hline НОМО & -10 & (\# & 48) & 0.002 & 0.002 \\
\hline HOMO & -9 & (\# & 49) & 0.001 & 0.02 \\
\hline HOMO & -8 & (\# & 50) & 0.001 & 0.00 \\
\hline HOMO & -7 & (\# & 51) & 0.066 & 0.00 \\
\hline HOMO & -6 & (\# & 52) & -0.001 & 0.00 \\
\hline
\end{tabular}




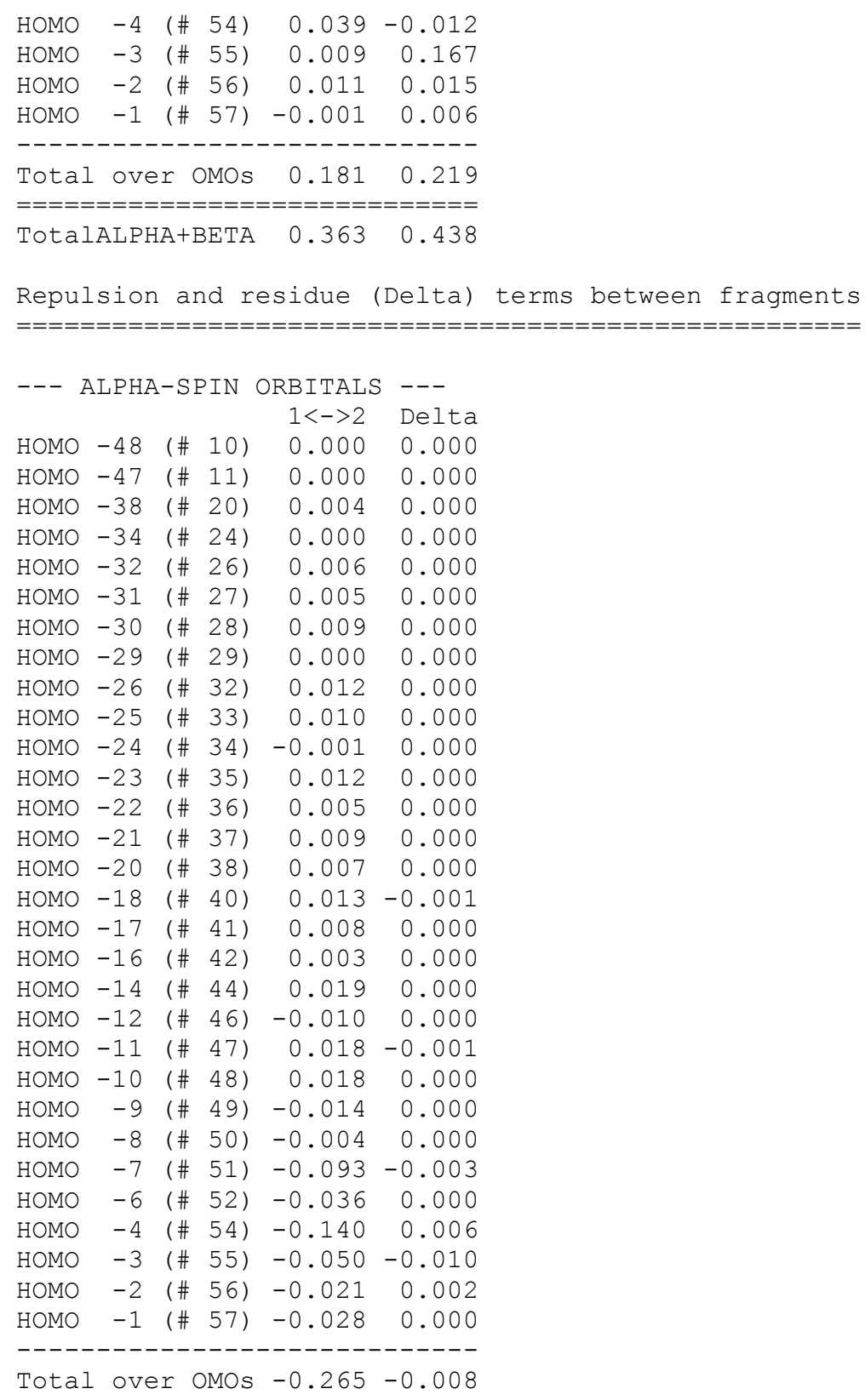

ONIOM optimization and CDA calculations for 2ar. 


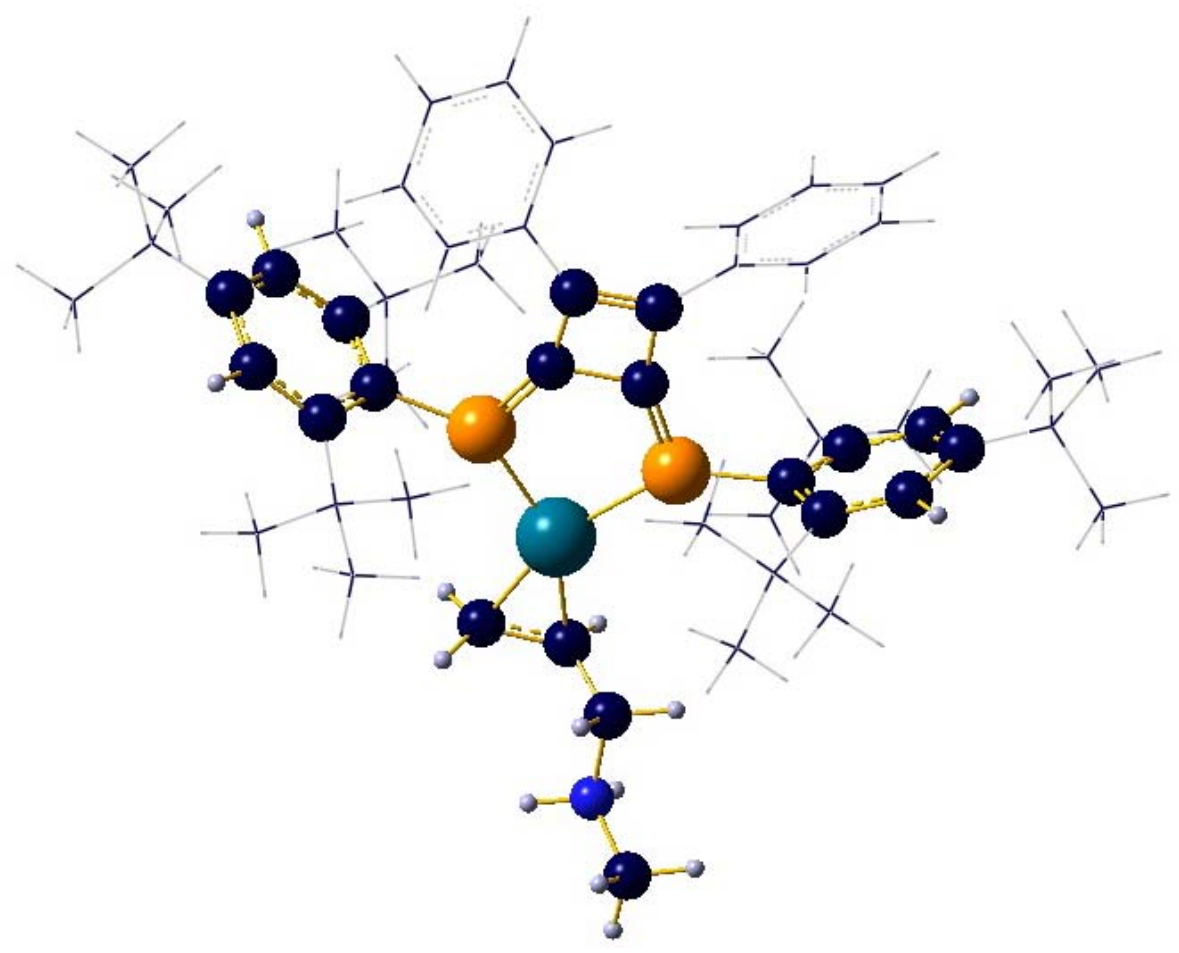

\begin{tabular}{|c|c|c|c|c|c|}
\hline \multirow{2}{*}{$\begin{array}{l}\text { Center } \\
\text { Number }\end{array}$} & \multirow{2}{*}{$\begin{array}{l}\text { Atomic } \\
\text { Number }\end{array}$} & \multirow{2}{*}{$\begin{array}{c}\text { Atomic } \\
\text { Type }\end{array}$} & \multicolumn{3}{|c|}{ Coordinates (Angstroms) } \\
\hline & & & $\mathrm{X}$ & $\mathrm{Y}$ & Z \\
\hline 1 & 6 & 10061003 & 1.546522 & 4.991852 & -.381649 \\
\hline 2 & 6 & 10061003 & -.730425 & 4.481191 & .620094 \\
\hline 3 & 6 & 10061003 & .687646 & 4.501485 & .729409 \\
\hline 4 & 1 & 10011000 & 2.573097 & 4.617165 & -.342029 \\
\hline 5 & 1 & 10011000 & -1.344471 & 4.567164 & 1.514073 \\
\hline 6 & 1 & 10011000 & 1.138033 & 4.554096 & 1.722738 \\
\hline 7 & 1 & 10011000 & -1.220865 & 4.806489 & -.299498 \\
\hline 8 & 1 & 10011000 & 1.123503 & 4.791410 & -1.369730 \\
\hline 9 & 7 & 10071003 & 1.681070 & 6.537810 & -.317810 \\
\hline 10 & 1 & 10011000 & .727785 & 6.915749 & -.312830 \\
\hline 11 & 1 & 10011000 & 2.070134 & 6.777324 & .599644 \\
\hline 12 & 6 & 10061003 & 2.491792 & 7.161811 & -1.401577 \\
\hline 13 & 1 & 10011000 & 2.524902 & 8.243989 & -1.260385 \\
\hline 14 & 1 & 10011000 & 3.501031 & 6.748369 & -1.364205 \\
\hline 15 & 1 & 10011000 & 2.031375 & 6.923809 & -2.361808 \\
\hline 16 & 46 & 10461004 & .012153 & 2.515174 & .379624 \\
\hline 17 & 15 & 10151003 & -1.630546 & .841363 & .134103 \\
\hline 18 & 6 & 10061000 & -.866067 & -.643115 & -.005782 \\
\hline 19 & 15 & 10151003 & 1.539398 & .716258 & .183532 \\
\hline 20 & 6 & 10061000 & .662770 & -.708762 & .081055 \\
\hline 21 & 6 & 10061000 & -.841455 & -2.094547 & -.133079 \\
\hline 22 & 6 & 10061000 & .529554 & -2.155392 & -.027294 \\
\hline 23 & 6 & 10061000 & 3.342944 & .319577 & .044902 \\
\hline 24 & 6 & 10061000 & 4.126843 & .132474 & 1.236753 \\
\hline 25 & 6 & 10061000 & 3.906207 & .046086 & -1.252860 \\
\hline 26 & 6 & 10061000 & 5.298918 & -.640474 & 1.106240 \\
\hline 27 & 6 & 10061000 & 5.115959 & -.672090 & -1.266757 \\
\hline 28 & 6 & 10061000 & 5.789119 & -1.108597 & -.118615 \\
\hline 29 & 1 & 10011000 & 5.843192 & -.906451 & 1.999867 \\
\hline 30 & 1 & 10011000 & 5.575994 & -.944629 & -2.207398 \\
\hline 31 & 6 & 10061000 & -3.463287 & .603324 & .159013 \\
\hline 32 & 6 & 10061000 & -4.104791 & .184441 & 1.380908 \\
\hline 33 & 6 & 10061000 & -4.209275 & .715527 & -1.067376 \\
\hline 34 & 6 & 10061000 & -5.402917 & -.351943 & 1.258190 \\
\hline 35 & 6 & 10061000 & -5.491361 & .135429 & -1.070187 \\
\hline
\end{tabular}




\begin{tabular}{|c|c|c|c|c|c|}
\hline 36 & 6 & 10061000 & -6.105431 & -.436064 & .050756 \\
\hline 37 & 1 & 10011000 & -5.890562 & -.742970 & 2.137383 \\
\hline 38 & 1 & 10011000 & -6.059947 & .094593 & -1.989870 \\
\hline 39 & 6 & 10061000 & -1.909750 & -3.099906 & -.274349 \\
\hline 40 & 6 & 10061000 & -1.797115 & -4.355727 & .342543 \\
\hline 41 & 6 & 10061000 & -3.042059 & -2.810379 & -1.047295 \\
\hline 42 & 6 & 10061000 & -2.803696 & -5.312301 & .176942 \\
\hline 43 & 1 & 10011000 & -.938674 & -4.591601 & .958687 \\
\hline 44 & 6 & 10061000 & -4.035219 & -3.775432 & -1.229656 \\
\hline 45 & 1 & 10011000 & -3.137529 & -1.854588 & -1.540058 \\
\hline 46 & 6 & 10061000 & -3.920511 & -5.023907 & -.612676 \\
\hline 47 & 1 & 10011000 & -2.714923 & -6.279027 & .655415 \\
\hline 48 & 1 & 10011000 & -4.884811 & -3.559808 & -1.862539 \\
\hline 49 & 1 & 10011000 & -4.693117 & -5.769013 & -.751289 \\
\hline 50 & 6 & 10061000 & 1.518630 & -3.248166 & -.046580 \\
\hline 51 & 6 & 10061000 & 1.324194 & -4.375416 & -.859907 \\
\hline 52 & 6 & 10061000 & 2.663234 & -3.167255 & .757417 \\
\hline 53 & 6 & 10061000 & 2.264567 & -5.410515 & -.861800 \\
\hline 54 & 1 & 10011000 & .455181 & -4.448033 & -1.501574 \\
\hline 55 & 6 & 10061000 & 3.594515 & -4.208234 & .764452 \\
\hline 56 & 1 & 10011000 & 2.817958 & -2.315433 & 1.401830 \\
\hline 57 & 6 & 10061000 & 3.398311 & -5.328013 & -.048324 \\
\hline 58 & 1 & 10011000 & 2.114091 & -6.276456 & -1.493296 \\
\hline 59 & 1 & 10011000 & 4.468399 & -4.146050 & 1.399854 \\
\hline 60 & 1 & 10011000 & 4.124194 & -6.130748 & -.047691 \\
\hline 61 & 6 & 10061003 & -3.707692 & 1.494437 & -2.334351 \\
\hline 62 & 6 & 10061003 & -3.481376 & .400713 & 2.807908 \\
\hline 63 & 6 & 10061003 & -2.673810 & .654421 & -3.116482 \\
\hline 64 & 1 & 10011000 & -1.758598 & .446811 & -2.534905 \\
\hline 65 & 1 & 10011000 & -3.117189 & -.317911 & -3.419798 \\
\hline 66 & 1 & 10011000 & -2.355169 & 1.189369 & -4.037474 \\
\hline 67 & 6 & 10061003 & -3.094432 & 2.865113 & -1.937462 \\
\hline 68 & 1 & 10011000 & -3.687144 & 3.339424 & -1.125790 \\
\hline 69 & 1 & 10011000 & -2.039677 & 2.783365 & -1.619185 \\
\hline 70 & 1 & 10011000 & -3.068338 & 3.571772 & -2.794811 \\
\hline 71 & 6 & 10061003 & -4.857558 & 1.819512 & -3.331937 \\
\hline 72 & 1 & 10011000 & -5.689442 & 2.346865 & -2.816212 \\
\hline 73 & 1 & 10011000 & -4.503635 & 2.473246 & -4.158859 \\
\hline 74 & 1 & 10011000 & -5.244426 & .902891 & -3.826564 \\
\hline 75 & 6 & 10061003 & -7.529683 & -1.034084 & -.071716 \\
\hline 76 & 6 & 10061003 & -8.506512 & .051490 & -.578900 \\
\hline 77 & 1 & 10011000 & -8.260950 & .380405 & -1.609710 \\
\hline 78 & 1 & 10011000 & -9.549387 & -.333169 & -.599357 \\
\hline 79 & 1 & 10011000 & -8.476163 & .939648 & .088775 \\
\hline 80 & 6 & 10061003 & -7.516180 & -2.215210 & -1.064700 \\
\hline 81 & 1 & 10011000 & -7.152964 & -1.909940 & -2.067714 \\
\hline 82 & 1 & 10011000 & -6.862071 & -3.026619 & -.682820 \\
\hline 83 & 1 & 10011000 & -8.538674 & -2.632278 & -1.192872 \\
\hline 84 & 6 & 10061003 & -8.089781 & -1.567994 & 1.270003 \\
\hline 85 & 1 & 10011000 & -8.179155 & -.749753 & 2.017155 \\
\hline 86 & 1 & 10011000 & -9.104768 & -1.999893 & 1.129849 \\
\hline 87 & 1 & 10011000 & -7.441867 & -2.373475 & 1.678959 \\
\hline 88 & 6 & 10061003 & -4.469243 & .071544 & 3.964002 \\
\hline 89 & 1 & 10011000 & -4.025370 & .318083 & 4.953642 \\
\hline 90 & 1 & 10011000 & -5.404744 & .665668 & 3.872994 \\
\hline 91 & 1 & 10011000 & -4.710610 & -1.013501 & 3.988645 \\
\hline 92 & 6 & 10061003 & -3.104055 & 1.890626 & 2.999365 \\
\hline 93 & 1 & 10011000 & -2.297770 & 2.224462 & 2.323555 \\
\hline 94 & 1 & 10011000 & -3.990056 & 2.537345 & 2.819836 \\
\hline 95 & 1 & 10011000 & -2.739554 & 2.077419 & 4.032928 \\
\hline 96 & 6 & 10061003 & -2.239843 & -.502189 & 3.026199 \\
\hline 97 & 1 & 10011000 & -2.382938 & -1.489013 & 2.535675 \\
\hline 98 & 1 & 10011000 & -1.305889 & -.038302 & 2.657559 \\
\hline 99 & 1 & 10011000 & -2.052071 & -.687080 & 4.106260 \\
\hline 100 & 6 & 10061003 & 3.780325 & .792474 & 2.616133 \\
\hline 101 & 6 & 10061003 & 4.998735 & .830099 & 3.584002 \\
\hline 102 & 1 & 10011000 & 5.881007 & 1.290959 & 3.088821 \\
\hline 103 & 1 & 10011000 & 4.773460 & 1.424474 & 4.496285 \\
\hline
\end{tabular}




$\begin{array}{rrrrrr}104 & 1 & 10011000 & 5.264102 & -.183738 & 3.952757 \\ 105 & 6 & 10061003 & 2.666849 & .000146 & 3.335329 \\ 106 & 1 & 10011000 & 1.711344 & .002191 & 2.781490 \\ 107 & 1 & 10011000 & 2.978459 & -1.056017 & 3.481785 \\ 108 & 1 & 10011000 & 2.460938 & .439949 & 4.335362 \\ 109 & 6 & 10061003 & 3.349323 & 2.271909 & 2.428319 \\ 110 & 1 & 10011000 & 2.301065 & 2.371140 & 2.098843 \\ 111 & 1 & 10011000 & 3.395710 & 2.838783 & 3.383128 \\ 112 & 1 & 10011000 & 4.011344 & 2.782167 & 1.695766 \\ 113 & 6 & 10061003 & 3.300523 & .588552 & -2.595292 \\ 114 & 6 & 10061003 & 4.244402 & .379890 & -3.813504 \\ 115 & 1 & 10011000 & 3.829342 & .855849 & -4.729061 \\ 116 & 1 & 10011000 & 5.240019 & .839969 & -3.630603 \\ 117 & 1 & 10011000 & 4.364949 & -.700344 & -4.047149 \\ 118 & 6 & 10061003 & 1.970188 & -.126252 & -2.943343 \\ 119 & 1 & 10011000 & 2.022600 & -1.202839 & -2.672528 \\ 120 & 1 & 10011000 & 1.096203 & .335215 & -2.447294 \\ 121 & 1 & 10011000 & 1.744866 & -.060694 & -4.030023 \\ 122 & 6 & 10061003 & 3.078244 & 2.117940 & -2.497295 \\ 123 & 1 & 10011000 & 2.303162 & 2.393502 & -1.761441 \\ 124 & 1 & 10011000 & 4.023159 & 2.627093 & -2.208593 \\ 125 & 1 & 10011000 & 2.741250 & 2.533676 & -3.471703 \\ 126 & 6 & 10061003 & 7.069438 & -1.967685 & -.256745 \\ 127 & 6 & 10061003 & 7.626818 & -2.450351 & 1.104877 \\ 128 & 6 & 10061003 & 6.778918 & -3.227525 & -1.105706 \\ 129 & 6 & 10061003 & 8.169379 & -1.133422 & -.947421 \\ 130 & 1 & 10011000 & 7.969476 & -1.593613 & 1.724393 \\ 131 & 1 & 10011000 & 6.857273 & -3.025916 & 1.663418 \\ 132 & 1 & 10011000 & 8.505836 & -3.115343 & .959128 \\ 133 & 1 & 10011000 & 6.496699 & -2.973736 & -2.147798 \\ 134 & 1 & 10011000 & 7.677620 & -3.879104 & -1.164930 \\ 135 & 1 & 10011000 & 5.955641 & -3.817562 & -.655926 \\ 136 & 1 & 10011000 & 9.115974 & -1.711964 & -1.019370 \\ 137 & 1 & 10011000 & 7.875559 & -.843635 & -1.978008 \\ 138 & 1 & 10011000 & 8.370225 & -.205343 & -.369557 \\ -------------1\end{array}$

Electron donation between fragments $(<0.001$ e for any omitted MO)

\begin{tabular}{|c|c|c|c|}
\hline & & $1->2$ & $2->1$ \\
\hline HOMO-226 & (\# & 0.002 & 0.000 \\
\hline HOMO-216 & (\# 18) & 0.002 & 0.000 \\
\hline HOMO-166 & (\# 68) & 0.000 & 0.001 \\
\hline HOMO-165 & $\left(\begin{array}{l}\# \text { 69) } \\
\text { (1) }\end{array}\right.$ & -0.001 & 0.002 \\
\hline HOMO-164 & $(\#$ \# 70) & 0.000 & 0.002 \\
\hline HOMO-162 & $\left(\begin{array}{l}\# \text { 72) } \\
\text { (3) }\end{array}\right.$ & 0.008 & 0.000 \\
\hline HOMO-156 & $(\#$ (\# 78) & 0.015 & 0.00 \\
\hline HOMO-151 & (\# 83) & 0.012 & 0.00 \\
\hline HOMO-144 & $\left(\begin{array}{l}\# \text { 90) } \\
\text { (1) }\end{array}\right.$ & 0.021 & 0.000 \\
\hline HOMO-143 & (\# 91) & 0.002 & 0.000 \\
\hline HOMO-125 & (\#109) & 0.001 & 0.000 \\
\hline HOMO- 122 & (\#112) & 0.003 & 0.000 \\
\hline HOMO-114 & $(\# 120)$ & 0.005 & 0.000 \\
\hline HOMO-111 & $(\# 123)$ & 0.005 & 0.000 \\
\hline HOMO-103 & (\#131) & 0.004 & 0.000 \\
\hline HOMO-101 & (\#133) & 0.001 & 0.000 \\
\hline $\mathrm{HOMO}-99$ & (\#135) & 0.004 & 0.000 \\
\hline HOMO -96 & (\#138) & 0.004 & 0.001 \\
\hline $\mathrm{HOMO}-86$ & (\#148) & 0.001 & 0.000 \\
\hline HOMO -84 & $(\# 150)$ & 0.002 & 0.000 \\
\hline $\mathrm{HOMO}-83$ & (\#151) & 0.006 & 0.000 \\
\hline HOMO -81 & (\#153) & 0.003 & 0.001 \\
\hline HOMO -64 & $(\# 170)$ & 0.006 & 0.000 \\
\hline $\mathrm{HOMO}-56$ & (\#178) & 0.001 & -0.001 \\
\hline HOMO -47 & (\#187) & 0.000 & 0.002 \\
\hline HOMO -45 & (\#189) & 0.000 & 0.00 \\
\hline HOMO -44 & (\#190) & 0.001 & 0.004 \\
\hline HOMO -43 & (\#191) & 0.003 & 0.00 \\
\hline
\end{tabular}




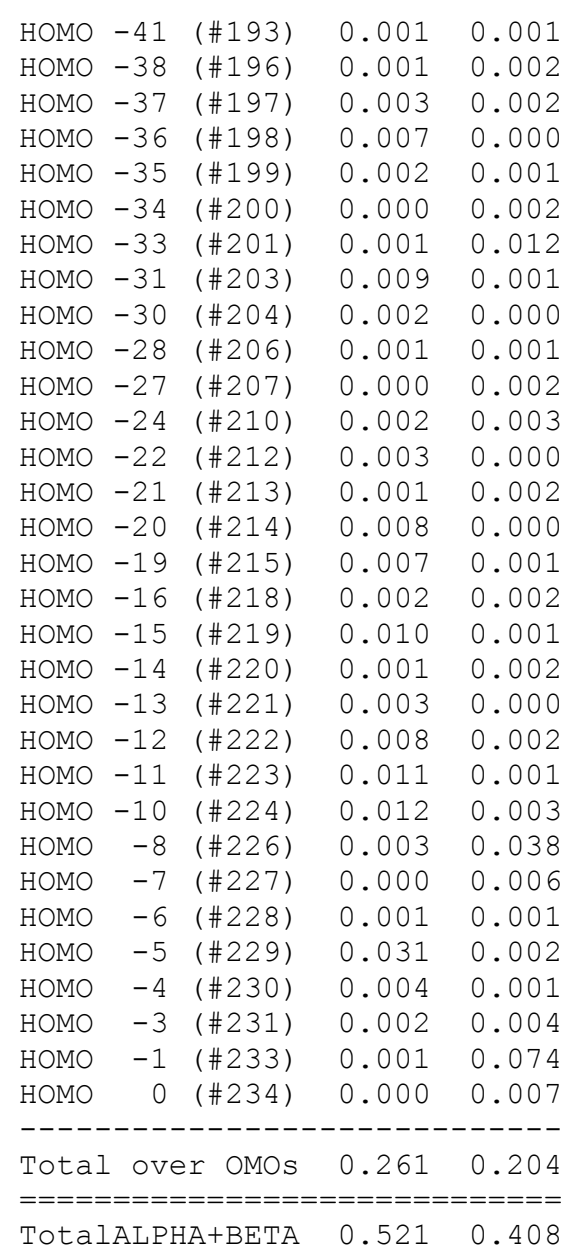

Repulsion and residue (Delta) terms between fragments

\begin{tabular}{|c|c|c|c|}
\hline--- ALPHA & -SPIN & ORBITALS & --- \\
\hline HOMO-226 & $\left(\begin{array}{ll}\# & 8\end{array}\right)$ & 0.000 & 0.000 \\
\hline HOMO-216 & $\left(\begin{array}{ll}\# & 18\end{array}\right)$ & 0.000 & 0.000 \\
\hline HOMO-166 & $\left(\begin{array}{ll}\# \quad 68\end{array}\right)$ & 0.002 & 0.000 \\
\hline HOMO-165 & $\left(\begin{array}{ll}\# & 69\end{array}\right)$ & 0.006 & 0.000 \\
\hline HOMO-164 & $\left(\begin{array}{ll}\# & 70\end{array}\right)$ & 0.000 & 0.000 \\
\hline HOMO-162 & $\left(\begin{array}{ll}\# & 72\end{array}\right)$ & 0.000 & 0.000 \\
\hline HOMO-156 & $\left(\begin{array}{ll}\# \text { 78) } \\
\text { (1) }\end{array}\right.$ & 0.003 & 0.000 \\
\hline HOMO-151 & $\left(\begin{array}{ll}\# \quad 83\end{array}\right)$ & 0.002 & 0.000 \\
\hline HOMO-144 & $\left(\begin{array}{ll}\# & 90\end{array}\right)$ & 0.009 & 0.000 \\
\hline HOMO-143 & $\left(\begin{array}{ll}\# & 91\end{array}\right)$ & 0.000 & 0.000 \\
\hline HOMO-125 & (\#109) & 0.000 & 0.000 \\
\hline HOMO-122 & (\#112) & 0.014 & 0.000 \\
\hline HOMO-114 & (\#120) & 0.007 & 0.000 \\
\hline HOMO-111 & (\#123) & 0.005 & 0.000 \\
\hline HOMO-103 & (\#131) & 0.005 & 0.000 \\
\hline HOMO-101 & (\#133) & 0.002 & 0.000 \\
\hline HOMO -99 & (\#135) & 0.002 & 0.000 \\
\hline HOMO -96 & (\#138) & 0.015 & 0.000 \\
\hline HOMO -86 & (\#148) & 0.003 & 0.000 \\
\hline HOMO -84 & (\#150) & 0.006 & 0.000 \\
\hline HOMO -83 & (\#151) & 0.011 & 0.000 \\
\hline HOMO -81 & (\#153) & 0.005 & 0.000 \\
\hline HOMO -64 & (\#170) & 0.011 & 0.000 \\
\hline HOMO -56 & (\#178) & 0.005 & 0.000 \\
\hline HOMO -47 & (\#187) & -0.001 & 0.000 \\
\hline HOMO -45 & (\#189) & -0.001 & 0.000 \\
\hline HOMO -44 & (\#190) & -0.003 & 0.000 \\
\hline
\end{tabular}




\begin{tabular}{|c|c|c|c|c|}
\hline HOMO & -43 & (\#191) & 0.002 & 0.000 \\
\hline HOMO & -41 & (\#193) & -0.001 & 0.000 \\
\hline HOMO & -38 & (\#196) & -0.003 & 0.000 \\
\hline HOMO & -37 & (\#197) & 0.001 & .000 \\
\hline HOMO & -36 & (\#198) & 0.003 & .001 \\
\hline $\mathrm{OMO}$ & -35 & (\#199) & 0.001 & 000 \\
\hline $\mathrm{OMO}$ & -34 & (\#200) & 0.000 & 000 \\
\hline HOMO & -33 & (\#201) & -0.005 & 000 \\
\hline HOMO & -31 & (\#203) & -0.008 & -0. \\
\hline HOMO & -30 & (\#204) & -0.003 & 0.000 \\
\hline HOMO & -28 & (\#206) & -0.004 & 0.000 \\
\hline HOMO & -27 & (\#207) & -0.002 & 0.000 \\
\hline HOMO & -24 & (\#210) & -0.007 & 0.000 \\
\hline HOMO & -22 & (\#212) & -0.003 & 0.000 \\
\hline HOMO & -21 & (\#213) & -0.004 & 0.000 \\
\hline HOMO & -20 & (\#214) & -0.006 & 0.000 \\
\hline HOMO & -19 & (\#215) & -0.008 & 0.000 \\
\hline HOMO & -16 & (\#218) & -0.003 & 0.000 \\
\hline HOMO & -15 & (\#219) & -0.018 & 0.000 \\
\hline HOMO & -14 & (\#220) & -0.024 & 0.000 \\
\hline HOMO & -13 & (\#221) & -0.009 & 0.000 \\
\hline HOMO & -12 & (\#222) & -0.021 & 0.000 \\
\hline HOMO & -11 & (\#223) & -0.032 & 0.00 \\
\hline HOMO & -10 & (\#224) & -0.032 & 0.001 \\
\hline HOMO & -8 & (\#226) & -0.015 & 0.000 \\
\hline HOMO & -7 & (\#227) & -0.002 & 0.000 \\
\hline HOMO & -6 & (\#228) & -0.002 & 0.000 \\
\hline HOMO & -5 & (\#229) & -0.046 & 0.002 \\
\hline HOMO & -4 & (\#230) & -0.006 & 0.000 \\
\hline HOMO & -3 & (\#231) & -0.006 & 0.000 \\
\hline HOMO & -1 & (\#233) & -0.017 & 0.003 \\
\hline HOMO & 0 & (\#234) & -0.008 & 0.001 \\
\hline
\end{tabular}

ONIOM optimization and CDA calculations for $\mathbf{2} \mathbf{b r}$.

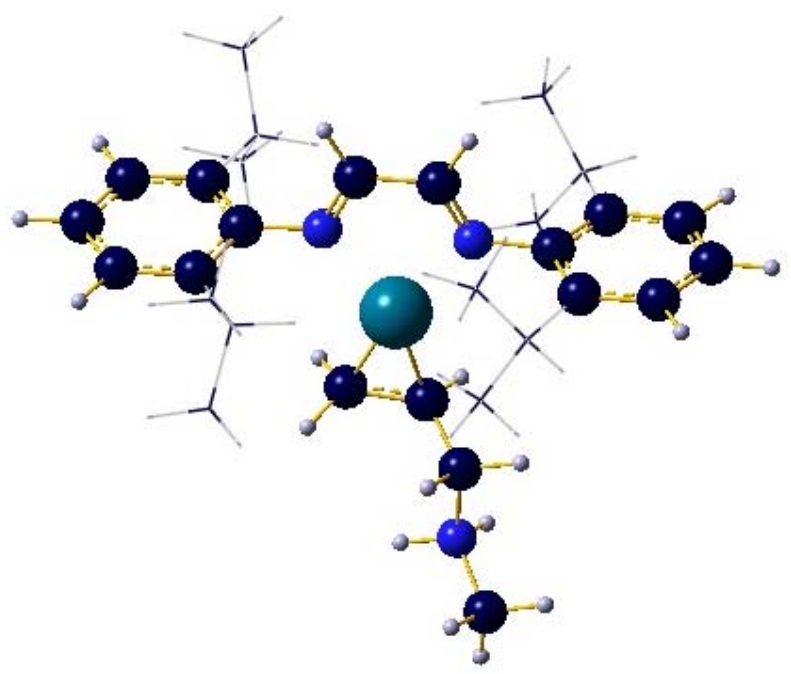

Standard orientation:

\begin{tabular}{|c|c|c|c|c|c|}
\hline \multirow{2}{*}{$\begin{array}{l}\text { Center } \\
\text { Number }\end{array}$} & \multirow{2}{*}{$\begin{array}{l}\text { Atomic } \\
\text { Number }\end{array}$} & \multirow{2}{*}{$\begin{array}{c}\text { Atomic } \\
\text { Type }\end{array}$} & \multicolumn{3}{|c|}{ Coordinates (Angstroms) } \\
\hline & & & $\mathrm{X}$ & $\mathrm{Y}$ & Z \\
\hline 1 & 6 & 10061003 & -1.746822 & 2.728518 & -1.716348 \\
\hline 2 & 6 & 10061003 & 0.569847 & 1.752328 & -2.091866 \\
\hline 3 & 6 & 10061003 & -0.857276 & 1.632045 & -2.184800 \\
\hline 4 & 1 & 10011000 & -2.768553 & 2.406407 & -1.495296 \\
\hline
\end{tabular}




\begin{tabular}{|c|c|c|c|c|c|}
\hline 5 & 1 & 10011000 & 1.202870 & 1.279711 & -2.842577 \\
\hline 6 & 1 & 10011000 & -1.280596 & 1.020033 & -2.985713 \\
\hline 7 & 1 & 10011000 & 1.017762 & 2.639454 & -1.634481 \\
\hline 8 & 1 & 10011000 & -1.339887 & 3.271482 & -0.859022 \\
\hline 9 & 7 & 10071003 & -1.894890 & 3.804812 & -2.823392 \\
\hline 10 & 1 & 10011000 & -0.943244 & 4.086657 & -3.082199 \\
\hline 11 & 1 & 10011000 & -2.273454 & 3.342150 & -3.655872 \\
\hline 12 & 6 & 10061003 & -2.721519 & 4.993489 & -2.470955 \\
\hline 13 & 1 & 10011000 & -2.758625 & 5.680908 & -3.318448 \\
\hline 14 & 1 & 10011000 & -3.728015 & 4.656027 & -2.218207 \\
\hline 15 & 1 & 10011000 & -2.270835 & 5.487061 & -1.608482 \\
\hline 16 & 46 & 10461004 & -0.075804 & 0.423215 & -0.674984 \\
\hline 17 & 7 & 10071000 & 1.437742 & -0.587025 & 0.486468 \\
\hline 18 & 7 & 10071000 & -1.184629 & -0.995490 & 0.651962 \\
\hline 19 & 6 & 10061000 & 1.031242 & -1.526164 & 1.261567 \\
\hline 20 & 1 & 10011000 & 1.718133 & -2.130588 & 1.857824 \\
\hline 21 & 6 & 10061000 & -0.415138 & -1.747820 & 1.352422 \\
\hline 22 & 1 & 10011000 & -0.805610 & -2.517891 & 2.022196 \\
\hline 23 & 6 & 10061000 & 2.837608 & -0.299867 & 0.412983 \\
\hline 24 & 6 & 10061000 & 3.660018 & -1.043144 & -0.462629 \\
\hline 25 & 6 & 10061000 & 3.350657 & 0.750873 & 1.207508 \\
\hline 26 & 6 & 10061000 & 5.032034 & -0.749686 & -0.477330 \\
\hline 27 & 6 & 10061000 & 4.729623 & 0.997154 & 1.153840 \\
\hline 28 & 6 & 10061000 & 5.563303 & 0.251647 & 0.326906 \\
\hline 29 & 1 & 10011000 & 5.708733 & -1.306244 & -1.121061 \\
\hline 30 & 1 & 10011000 & 5.175506 & 1.778511 & 1.764552 \\
\hline 31 & 1 & 10011000 & 6.629829 & 0.456924 & 0.304665 \\
\hline 32 & 6 & 10061000 & -2.598632 & -1.156681 & 0.771356 \\
\hline 33 & 6 & 10061000 & -3.305630 & -0.385417 & 1.734974 \\
\hline 34 & 6 & 10061000 & -3.283023 & -2.050224 & -0.096141 \\
\hline 35 & 6 & 10061000 & -4.686043 & -0.603640 & 1.864148 \\
\hline 36 & 6 & 10061000 & -4.664242 & -2.216020 & 0.089903 \\
\hline 37 & 6 & 10061000 & -5.359740 & -1.518543 & 1.067314 \\
\hline 38 & 1 & 10011000 & -5.264274 & -0.056086 & 2.606019 \\
\hline 39 & 1 & 10011000 & -5.225120 & -2.909064 & -0.534178 \\
\hline 40 & 1 & 10011000 & -6.426480 & -1.676862 & 1.197837 \\
\hline 41 & 6 & 10061003 & 2.448113 & 1.599574 & 2.099436 \\
\hline 42 & 1 & 10011000 & 1.386153 & 1.296177 & 1.991085 \\
\hline 43 & 6 & 10061003 & 2.508809 & 3.079286 & 1.701514 \\
\hline 44 & 1 & 10011000 & 3.517184 & 3.505886 & 1.886346 \\
\hline 45 & 1 & 10011000 & 2.261366 & 3.193409 & 0.624595 \\
\hline 46 & 1 & 10011000 & 1.767605 & 3.661391 & 2.289951 \\
\hline 47 & 6 & 10061003 & 2.804402 & 1.410498 & 3.578031 \\
\hline 48 & 1 & 10011000 & 2.744382 & 0.334063 & 3.846927 \\
\hline 49 & 1 & 10011000 & 3.829264 & 1.779374 & 3.794750 \\
\hline 50 & 1 & 10011000 & 2.085583 & 1.966990 & 4.216860 \\
\hline 51 & 6 & 10061003 & 3.091280 & -2.117870 & -1.384382 \\
\hline 52 & 1 & 10011000 & 1.989310 & -2.189800 & -1.271910 \\
\hline 53 & 6 & 10061003 & 3.346417 & -1.775387 & -2.857296 \\
\hline 54 & 1 & 10011000 & 4.431655 & -1.786340 & -3.092258 \\
\hline 55 & 1 & 10011000 & 2.837908 & -2.514629 & -3.512613 \\
\hline 56 & 1 & 10011000 & 2.937739 & -0.768569 & -3.088951 \\
\hline 57 & 6 & 10061003 & 3.658546 & -3.497638 & -1.032648 \\
\hline 58 & 1 & 10011000 & 4.753972 & -3.538955 & -1.210455 \\
\hline 59 & 1 & 10011000 & 3.458372 & -3.728505 & 0.035658 \\
\hline 60 & 1 & 10011000 & 3.170398 & -4.279057 & -1.653449 \\
\hline 61 & 6 & 10061003 & -2.674396 & 0.703577 & 2.608464 \\
\hline 62 & 1 & 10011000 & -3.478761 & 1.179321 & 3.214004 \\
\hline 63 & 6 & 10061003 & -2.069963 & 1.838131 & 1.773864 \\
\hline 64 & 1 & 10011000 & -1.759891 & 2.672512 & 2.438598 \\
\hline 65 & 1 & 10011000 & -1.178282 & 1.502180 & 1.209307 \\
\hline 66 & 1 & 10011000 & -2.827593 & 2.227284 & 1.061566 \\
\hline 67 & 6 & 10061003 & -1.668271 & 0.154071 & 3.628797 \\
\hline 68 & 1 & 10011000 & -0.654835 & 0.052934 & 3.194798 \\
\hline 69 & 1 & 10011000 & -1.582359 & 0.858369 & 4.483865 \\
\hline 70 & 1 & 10011000 & -2.005831 & -0.827881 & 4.023893 \\
\hline 71 & 6 & 10061003 & -2.626205 & -2.811208 & -1.250728 \\
\hline 72 & 1 & 10011000 & -3.421421 & -3.348330 & -1.815418 \\
\hline
\end{tabular}




\begin{tabular}{rrrrrr}
73 & 6 & 10061003 & -1.655828 & -3.900096 & -0.774763 \\
74 & 1 & 10011000 & -1.520153 & -4.654961 & -1.578761 \\
75 & 1 & 10011000 & -0.654085 & -3.488556 & -0.545133 \\
76 & 1 & 10011000 & -2.059021 & -4.418784 & 0.121098 \\
77 & 6 & 10061003 & -1.967059 & -1.873312 & -2.268361 \\
78 & 1 & 10011000 & -1.034923 & -1.428226 & -1.868840 \\
79 & 1 & 10011000 & -1.704904 & -2.439723 & -3.187405 \\
80 & 1 & 10011000 & -2.670205 & -1.060963 & -2.550320 \\
\hline
\end{tabular}

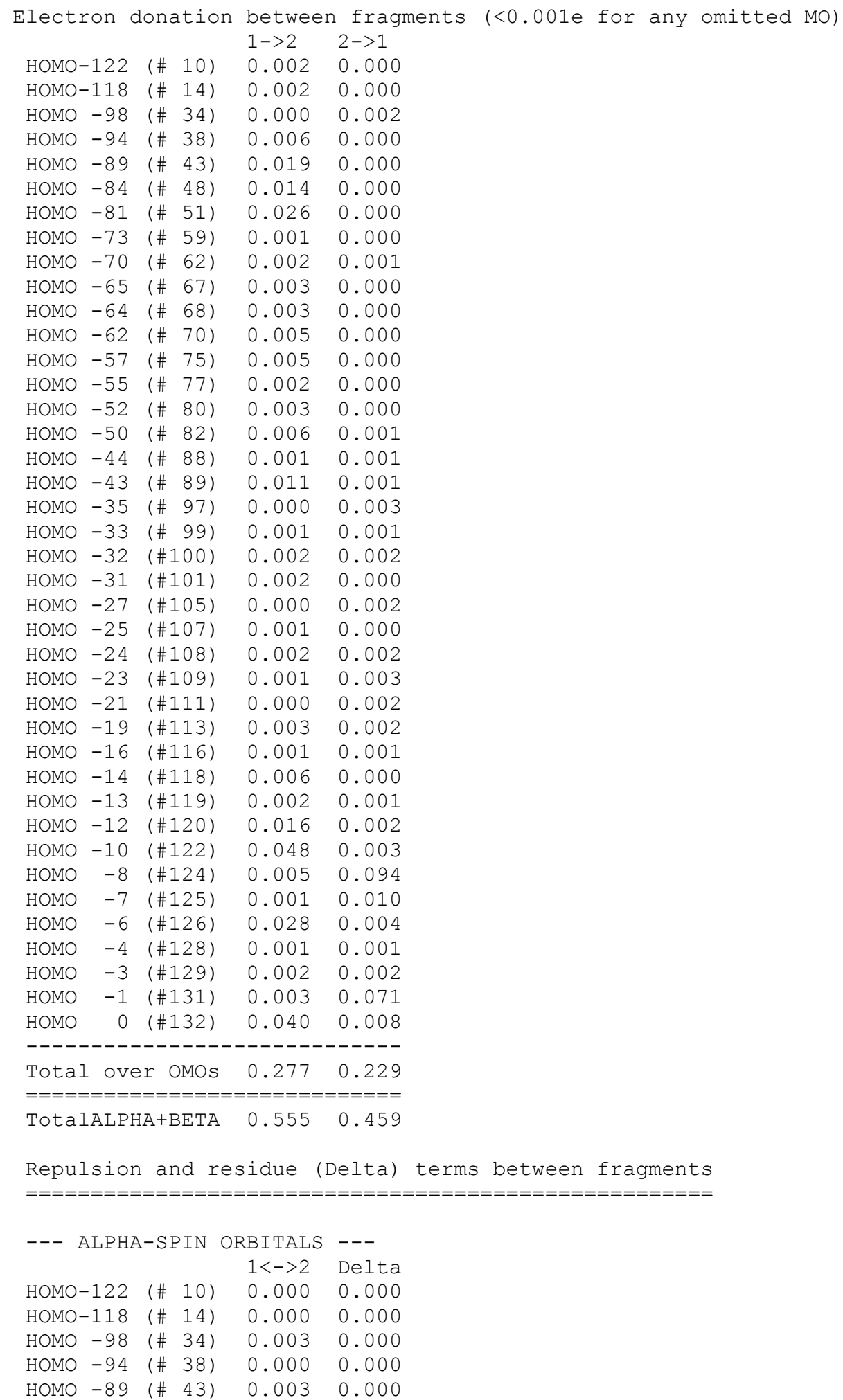




\begin{tabular}{|c|c|c|c|c|}
\hline HOMO & -84 & (\# 48) & 0.002 & 0.000 \\
\hline HOMO & -81 & (\# 51) & 0.010 & 0.000 \\
\hline HOMO & -73 & (\# 59) & 0.000 & 0.000 \\
\hline HOMO & -70 & (\# 62) & 0.015 & 0.000 \\
\hline HOMO & -65 & (\# 67) & 0.011 & 0.000 \\
\hline HOMO & -64 & (\# 68) & 0.001 & 0.000 \\
\hline HOMO & -62 & (\# 70) & 0.002 & 0.000 \\
\hline HOMO & -57 & (\# 75) & 0.010 & 0.000 \\
\hline HOMO & -55 & (\# 77) & 0.004 & 0.000 \\
\hline HOMO & -52 & (\# 80) & -0.002 & 0.000 \\
\hline HOMO & -50 & (\# 82) & 0.020 & 0.000 \\
\hline HOMO & -44 & (\# 88) & 0.006 & 0.000 \\
\hline HOMO & -43 & (\# 89) & 0.019 & -0.001 \\
\hline HOMO & -35 & (\# 97) & -0.002 & 0.000 \\
\hline HOMO & -33 & (\# 99) & 0.008 & 0.000 \\
\hline HOMO & -32 & (\#100) & 0.009 & 0.000 \\
\hline HOMO & -31 & (\#101) & -0.004 & 0.000 \\
\hline HOMO & -27 & (\#105) & -0.004 & 0.000 \\
\hline HOMO & -25 & (\#107) & 0.002 & 0.000 \\
\hline HOMO & -24 & (\#108) & 0.001 & 0.000 \\
\hline HOMO & -23 & (\#109) & 0.000 & 0.000 \\
\hline HOMO & -21 & (\#111) & -0.001 & 0.000 \\
\hline HOMO & -19 & (\#113) & -0.005 & 0.000 \\
\hline HOMO & -16 & (\#116) & -0.001 & 0.000 \\
\hline HOMO & -14 & (\#118) & 0.000 & 0.000 \\
\hline HOMO & -13 & (\#119) & -0.001 & 0.000 \\
\hline HOMO & -12 & (\#120) & -0.008 & -0.001 \\
\hline HOMO & -10 & (\#122) & -0.039 & -0.002 \\
\hline HOMO & -8 & (\#124) & -0.033 & -0.004 \\
\hline HOMO & -7 & (\#125) & -0.048 & 0.001 \\
\hline HOMO & -6 & (\#126) & -0.048 & 0.000 \\
\hline HOMO & -4 & (\#128) & -0.006 & 0.000 \\
\hline HOMO & -3 & (\#129) & -0.021 & 0.000 \\
\hline HOMO & -1 & (\#131) & -0.015 & -0.004 \\
\hline HOMO & 0 & (\#132) & -0.061 & 0.003 \\
\hline
\end{tabular}

Optimization and CDA calculations for $\mathbf{2 c r}$.

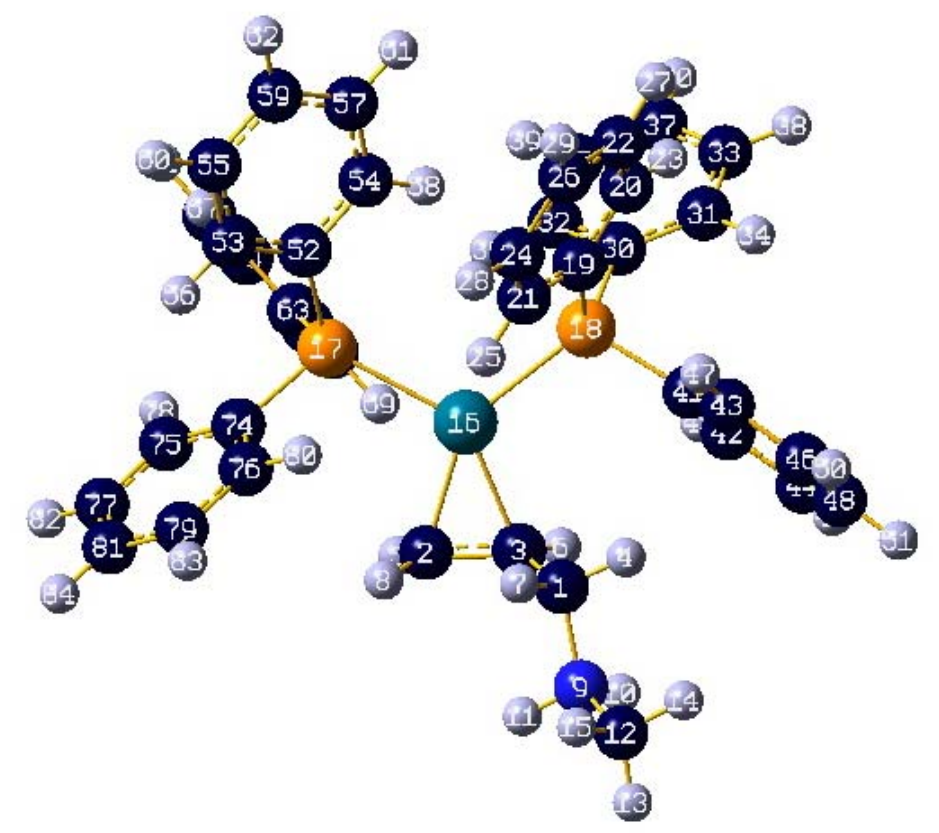

$\begin{array}{llc}\text { Center } & \text { Atomic } & \text { Atomic } \\ \text { Number } & \text { Number } & \text { Type }\end{array}$

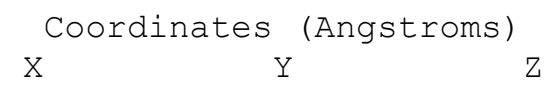




\begin{tabular}{|c|c|c|c|c|c|}
\hline 1 & 6 & 0 & -1.983701 & 3.286801 & -0.081127 \\
\hline 2 & 6 & 0 & 0.309066 & 2.941176 & -1.109261 \\
\hline 3 & 6 & 0 & -1.097241 & 2.717861 & -1.130424 \\
\hline 4 & 1 & 0 & -2.912905 & 2.729812 & 0.065850 \\
\hline 5 & 1 & 0 & 0.870684 & 2.954784 & -2.041436 \\
\hline 6 & 1 & 0 & -1.589094 & 2.495760 & -2.080253 \\
\hline 7 & 1 & 0 & -1.474558 & 3.415109 & 0.878282 \\
\hline 8 & 1 & 0 & 0.767143 & 3.519219 & -0.304493 \\
\hline 9 & 7 & 0 & -2.436461 & 4.715600 & -0.482205 \\
\hline 10 & 1 & 0 & -2.919537 & 4.637921 & -1.382581 \\
\hline 11 & 1 & 0 & -1.584945 & 5.253903 & -0.674515 \\
\hline 12 & 6 & 0 & -3.301043 & 5.420635 & 0.504898 \\
\hline 13 & 1 & 0 & -3.568076 & 6.408080 & 0.122599 \\
\hline 14 & 1 & 0 & -4.200054 & 4.823579 & 0.666254 \\
\hline 15 & 1 & 0 & -2.750600 & 5.519140 & 1.441883 \\
\hline 16 & 46 & 0 & -0.097686 & 0.965089 & -0.445445 \\
\hline 17 & 15 & 0 & 2.106489 & 0.170231 & -0.026695 \\
\hline 18 & 15 & 0 & -1.791736 & -0.648158 & -0.014206 \\
\hline 19 & 6 & 0 & -1.787004 & -1.430042 & 1.652006 \\
\hline 20 & 6 & 0 & -2.368941 & -2.674717 & 1.927792 \\
\hline 21 & 6 & 0 & -1.183848 & -0.716273 & 2.697186 \\
\hline 22 & 6 & 0 & -2.356848 & -3.186232 & 3.225586 \\
\hline 23 & 1 & 0 & -2.825252 & -3.254607 & 1.130604 \\
\hline 24 & 6 & 0 & -1.182862 & -1.222098 & 3.996921 \\
\hline 25 & 1 & 0 & -0.697040 & 0.233594 & 2.482574 \\
\hline 26 & 6 & 0 & -1.770409 & -2.459217 & 4.263112 \\
\hline 27 & 1 & 0 & -2.806675 & -4.155714 & 3.425689 \\
\hline 28 & 1 & 0 & -0.708573 & -0.659159 & 4.797105 \\
\hline 29 & 1 & 0 & -1.762859 & -2.860847 & 5.273295 \\
\hline 30 & 6 & 0 & -1.921689 & -2.032372 & -1.218741 \\
\hline 31 & 6 & 0 & -3.111744 & -2.749125 & -1.423336 \\
\hline 32 & 6 & 0 & -0.785377 & -2.379339 & -1.962359 \\
\hline 33 & 6 & 0 & -3.153776 & -3.803019 & -2.334966 \\
\hline 34 & 1 & 0 & -4.013835 & -2.476470 & -0.880790 \\
\hline 35 & 6 & 0 & -0.827738 & -3.436868 & -2.872175 \\
\hline 36 & 1 & 0 & 0.133292 & -1.810540 & -1.839391 \\
\hline 37 & 6 & 0 & -2.010583 & -4.151986 & -3.057464 \\
\hline 38 & 1 & 0 & -4.081427 & -4.350375 & -2.483365 \\
\hline 39 & 1 & 0 & 0.062792 & -3.693580 & -3.440234 \\
\hline 40 & 1 & 0 & -2.045962 & -4.973446 & -3.768609 \\
\hline 41 & 6 & 0 & -3.486559 & 0.091131 & -0.105168 \\
\hline 42 & 6 & 0 & -3.971990 & 0.514286 & -1.355038 \\
\hline 43 & 6 & 0 & -4.265837 & 0.334489 & 1.033476 \\
\hline 44 & 6 & 0 & -5.206228 & 1.153995 & -1.461833 \\
\hline 45 & 1 & 0 & -3.387905 & 0.323717 & -2.253392 \\
\hline 46 & 6 & 0 & -5.499471 & 0.985143 & 0.927186 \\
\hline 47 & 1 & 0 & -3.919274 & 0.004563 & 2.009362 \\
\hline 48 & 6 & 0 & -5.974838 & 1.395916 & -0.318060 \\
\hline 49 & 1 & 0 & -5.578888 & 1.446141 & -2.441396 \\
\hline 50 & 1 & 0 & -6.097177 & 1.149369 & 1.820930 \\
\hline 51 & 1 & 0 & -6.944976 & 1.879762 & -0.402583 \\
\hline 52 & 6 & 0 & 2.475858 & -1.133953 & 1.222158 \\
\hline 53 & 6 & 0 & 3.505768 & -1.016520 & 2.165403 \\
\hline 54 & 6 & 0 & 1.710379 & -2.308533 & 1.197808 \\
\hline 55 & 6 & 0 & 3.761704 & -2.053803 & 3.064707 \\
\hline 56 & 1 & 0 & 4.115864 & -0.118391 & 2.199709 \\
\hline 57 & 6 & 0 & 1.975393 & -3.348219 & 2.086385 \\
\hline 58 & 1 & 0 & 0.901706 & -2.414786 & 0.479262 \\
\hline 59 & 6 & 0 & 3.000705 & -3.221686 & 3.025757 \\
\hline 60 & 1 & 0 & 4.563807 & -1.948147 & 3.791264 \\
\hline
\end{tabular}




$\begin{array}{rrrrrr}61 & 1 & 0 & 1.374463 & -4.253327 & 2.050207 \\ 62 & 1 & 0 & 3.204221 & -4.029654 & 3.724097 \\ 63 & 6 & 0 & 2.916863 & -0.486656 & -1.544936 \\ 64 & 6 & 0 & 3.988753 & -1.389531 & -1.492806 \\ 65 & 6 & 0 & 2.448077 & -0.060794 & -2.796196 \\ 66 & 6 & 0 & 4.583363 & -1.846909 & -2.668993 \\ 67 & 1 & 0 & 4.358196 & -1.740956 & -0.532967 \\ 68 & 6 & 0 & 3.047862 & -0.513425 & -3.971655 \\ 69 & 1 & 0 & 1.603426 & 0.623499 & -2.844926 \\ 70 & 6 & 0 & 4.117208 & -1.407993 & -3.909556 \\ 71 & 1 & 0 & 5.412993 & -2.547554 & -2.614857 \\ 72 & 1 & 0 & 2.674902 & -0.173703 & -4.934944 \\ 73 & 1 & 0 & 4.582579 & -1.766397 & -4.824385 \\ 74 & 6 & 0 & 3.199118 & 1.560302 & 0.497493 \\ 75 & 6 & 0 & 4.361353 & 1.919307 & -0.194245 \\ 76 & 6 & 0 & 2.821532 & 2.312358 & 1.621848 \\ 77 & 6 & 0 & 5.132044 & 3.005071 & 0.230522 \\ 78 & 1 & 0 & 4.672684 & 1.352748 & -1.067172 \\ 79 & 6 & 0 & 3.599133 & 3.384434 & 2.054630 \\ 80 & 1 & 0 & 1.917456 & 2.048092 & 2.168240 \\ 81 & 6 & 0 & 4.757296 & 3.736813 & 1.355888 \\ 82 & 1 & 0 & 6.031824 & 3.271408 & -0.318760 \\ 83 & 1 & 0 & 3.303558 & 3.945265 & 2.938504 \\ 84 & 1 & 0 & 5.362998 & 4.575333 & 1.690241\end{array}$

Electron donation between fragments $(<0.001$ e for any omitted MO)

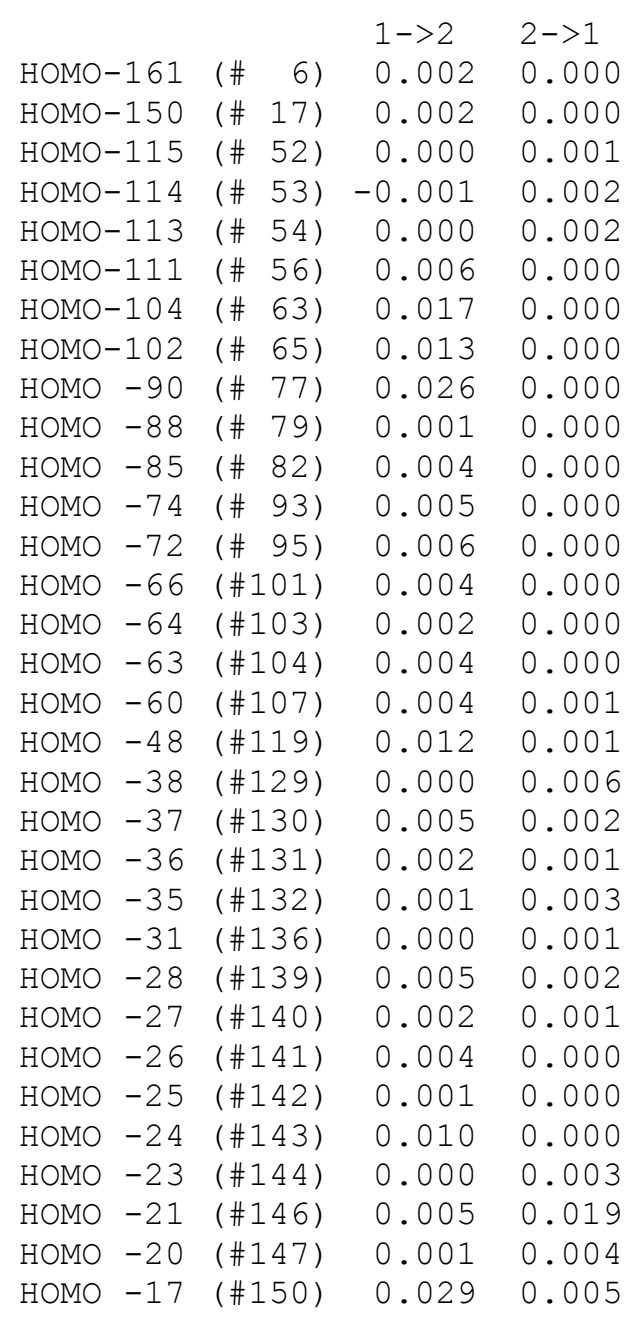




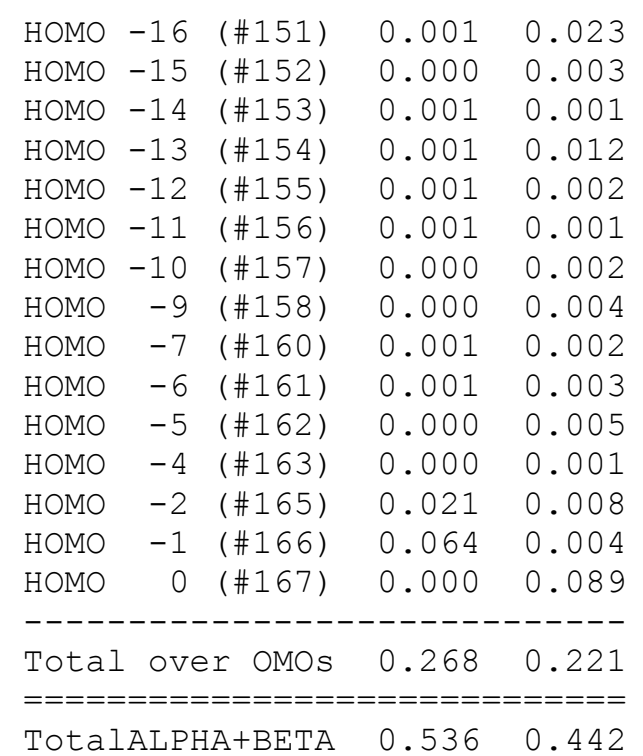

Repulsion and residue (Delta) terms between fragments

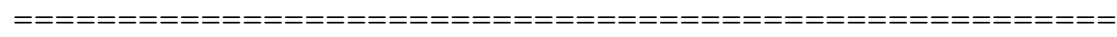

\begin{tabular}{|c|c|c|c|c|}
\hline & \multirow{2}{*}{\multicolumn{2}{|c|}{ ALPHA-SPIN }} & \multirow{2}{*}{$\begin{array}{l}\text { ORBITALS } \\
1<->?\end{array}$} & $5--$ \\
\hline & & & & Delta \\
\hline & & $\left(\begin{array}{ll}\# & 6\end{array}\right)$ & & \\
\hline & -150 & $\left(\begin{array}{l}\# \text { 17) } \\
\text { 17 }\end{array}\right.$ & & \\
\hline & -115 & $\left(\begin{array}{l}\# \quad 52\end{array}\right)$ & & \\
\hline & & (\# 53 & & \\
\hline & -113 & (\# 54 & & \\
\hline & -111 & (\# 56 & & \\
\hline & & (\# 63 & & \\
\hline & & (\# 65 & & \\
\hline & & (\# 77 & & \\
\hline & -88 & (\# 79 & & \\
\hline & -85 & (\# 82 & & \\
\hline & -74 & (\# 9 & & \\
\hline & -72 & (\# 9 & & \\
\hline & -66 & (\#10 & & \\
\hline & -64 & & & \\
\hline & -63 & & & \\
\hline & -60 & & & \\
\hline & -48 & & & \\
\hline & -38 & & & \\
\hline & -37 & & & \\
\hline & -36 & & & \\
\hline & -35 & & & \\
\hline & -31 & & & \\
\hline & -28 & & & \\
\hline & -27 & & & \\
\hline & -26 & & & \\
\hline & -25 & & & \\
\hline & -24 & & & -0 . \\
\hline & -23 & & & \\
\hline & -21 & & & -0 . \\
\hline & -20 & $(\# 14$ & & \\
\hline & -17 & & & \\
\hline & -16 & & & \\
\hline & -15 & & & \\
\hline & -14 & & & \\
\hline & -13 & & -0005 & \\
\hline
\end{tabular}




$\begin{array}{lrlll}\text { HOMO } & -12 & (\# 155) & -0.007 & 0.000 \\ \text { HOMO } & -11 & (\# 156) & -0.008 & 0.000 \\ \text { HOMO } & -10 & (\# 157) & -0.005 & 0.000 \\ \text { HOMO } & -9 & (\# 158) & -0.002 & 0.000 \\ \text { HOMO } & -7 & (\# 160) & -0.016 & 0.000 \\ \text { HOMO } & -6 & (\# 161) & -0.005 & 0.000 \\ \text { HOMO } & -5 & (\# 162) & -0.017 & 0.000 \\ \text { HOMO } & -4 & (\# 163) & -0.003 & 0.000 \\ \text { HOMO } & -2 & (\# 165) & -0.029 & 0.001 \\ \text { HOMO } & -1 & (\# 166) & -0.123 & 0.003 \\ \text { HOMO } & (\#) & (\# 167) & -0.029 & 0.005 \\ \text {------------------------ } & \\ \text { Total over OMOs } & -0.198 & 0.001\end{array}$

Optimization and CDA calculations for $\mathbf{2 d r}$.

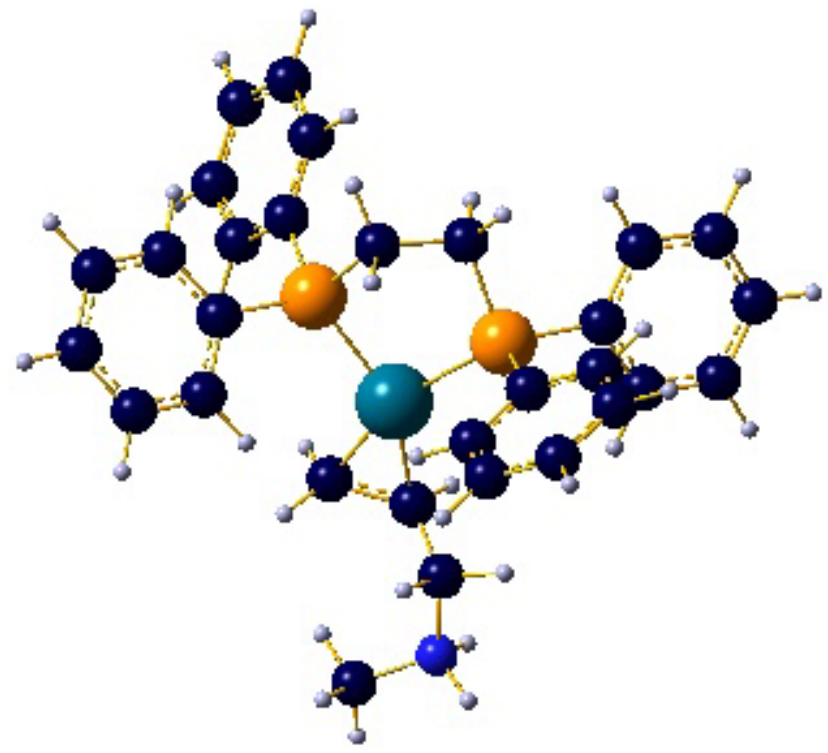

Standard orientation:

\begin{tabular}{|c|c|c|c|c|c|}
\hline \multirow{2}{*}{$\begin{array}{l}\text { Center } \\
\text { Number }\end{array}$} & \multirow{2}{*}{$\begin{array}{l}\text { Atomic } \\
\text { Number }\end{array}$} & \multirow{2}{*}{$\begin{array}{c}\text { Atomic } \\
\text { Type }\end{array}$} & \multicolumn{3}{|c|}{ Coordinates (Angstroms) } \\
\hline & & & $\mathrm{X}$ & $\mathrm{Y}$ & Z \\
\hline & & & & & \\
\hline 1 & 46 & 0 & -.010475 & .573144 & -.875417 \\
\hline 2 & 15 & 0 & -1.489267 & -.661274 & .505487 \\
\hline 3 & 6 & 0 & -.406180 & -1.916885 & 1.362868 \\
\hline 4 & 15 & 0 & 1.743291 & -.428471 & .327322 \\
\hline 5 & 6 & 0 & .923318 & -1.275097 & 1.770329 \\
\hline 6 & 1 & 0 & -.232847 & -2.736222 & .653873 \\
\hline 7 & 1 & 0 & -.916233 & -2.330526 & 2.241234 \\
\hline 8 & 1 & 0 & .752300 & -.486137 & 2.513460 \\
\hline 9 & 1 & 0 & 1.590946 & -2.005131 & 2.243780 \\
\hline 10 & 6 & 0 & .684945 & 1.834078 & -2.401214 \\
\hline 11 & 6 & 0 & -.744217 & 1.772241 & -2.479622 \\
\hline 12 & 1 & 0 & 1.280787 & 1.291679 & -3.133692 \\
\hline 13 & 6 & 0 & -1.610627 & 2.881100 & -2.017302 \\
\hline 14 & 1 & 0 & -1.201001 & 1.109400 & -3.218772 \\
\hline 15 & 1 & 0 & -2.610898 & 2.557503 & -1.714848 \\
\hline 16 & 1 & 0 & -1.165576 & 3.477212 & -1.215916 \\
\hline 17 & 1 & 0 & 1.174732 & 2.721355 & -1.996268 \\
\hline 18 & 7 & 0 & -1.898941 & 3.924530 & -3.162413 \\
\hline 19 & 1 & 0 & -2.656678 & 4.549940 & -2.869319 \\
\hline 20 & 1 & 0 & -2.254559 & 3.393407 & -3.963119 \\
\hline 21 & 6 & 0 & -.722407 & 4.730207 & -3.594409 \\
\hline 22 & 1 & 0 & .066992 & 4.042088 & -3.897275 \\
\hline
\end{tabular}




\begin{tabular}{|c|c|c|c|c|c|}
\hline 23 & 1 & 0 & -1.010006 & 5.376833 & -4.426031 \\
\hline 24 & 1 & 0 & -.386386 & 5.333078 & -2.749010 \\
\hline 25 & 6 & 0 & -2.890375 & -1.645499 & -.156293 \\
\hline 26 & 6 & 0 & -3.821977 & -.986416 & -.973511 \\
\hline 27 & 6 & 0 & -3.072717 & -3.010801 & .102210 \\
\hline 28 & 6 & 0 & -4.914150 & -1.667115 & -1.505408 \\
\hline 29 & 1 & 0 & -3.688280 & .071442 & -1.191221 \\
\hline 30 & 6 & 0 & -4.160315 & -3.697340 & -.442689 \\
\hline 31 & 1 & 0 & -2.375606 & -3.554749 & .733192 \\
\hline 32 & 6 & 0 & -5.083796 & -3.028786 & -1.244423 \\
\hline 33 & 1 & 0 & -5.631087 & -1.139225 & -2.129676 \\
\hline 34 & 1 & 0 & -4.284878 & -4.757044 & -.234361 \\
\hline 35 & 1 & 0 & -5.930355 & -3.564625 & -1.665666 \\
\hline 36 & 6 & 0 & -2.226400 & .255517 & 1.920585 \\
\hline 37 & 6 & 0 & -3.294251 & -.253140 & 2.674294 \\
\hline 38 & 6 & 0 & -1.668990 & 1.490906 & 2.279277 \\
\hline 39 & 6 & 0 & -3.786571 & .458331 & 3.768057 \\
\hline 40 & 1 & 0 & -3.750836 & -1.202317 & 2.403024 \\
\hline 41 & 6 & 0 & -2.156265 & 2.197914 & 3.379708 \\
\hline 42 & 1 & 0 & -.845337 & 1.893215 & 1.691138 \\
\hline 43 & 6 & 0 & -3.217346 & 1.682797 & 4.124665 \\
\hline 44 & 1 & 0 & -4.616948 & .055608 & 4.342771 \\
\hline 45 & 1 & 0 & -1.708341 & 3.150064 & 3.654137 \\
\hline 46 & 1 & 0 & -3.601664 & 2.233093 & 4.979743 \\
\hline 47 & 6 & 0 & 2.662975 & -1.744685 & -.562880 \\
\hline 48 & 6 & 0 & 3.369474 & -1.366928 & -1.716397 \\
\hline 49 & 6 & 0 & 2.683358 & -3.089607 & -.171759 \\
\hline 50 & 6 & 0 & 4.092215 & -2.305619 & -2.447932 \\
\hline 51 & 1 & 0 & 3.359162 & -.327147 & -2.036726 \\
\hline 52 & 6 & 0 & 3.397824 & -4.033303 & -.913738 \\
\hline 53 & 1 & 0 & 2.151052 & -3.421029 & .715462 \\
\hline 54 & 6 & 0 & 4.105015 & -3.644618 & -2.050073 \\
\hline 55 & 1 & 0 & 4.642798 & -1.993322 & -3.331833 \\
\hline 56 & 1 & 0 & 3.403048 & -5.073249 & -.596661 \\
\hline 57 & 1 & 0 & 4.662811 & -4.380008 & -2.623990 \\
\hline 58 & 6 & 0 & 3.061647 & .559924 & 1.135263 \\
\hline 59 & 6 & 0 & 4.235456 & -.028938 & 1.626953 \\
\hline 60 & 6 & 0 & 2.868765 & 1.937886 & 1.297660 \\
\hline 61 & 6 & 0 & 5.192075 & .748489 & 2.278450 \\
\hline 62 & 1 & 0 & 4.408820 & -1.093949 & 1.491893 \\
\hline 63 & 6 & 0 & 3.824129 & 2.713893 & 1.955036 \\
\hline 64 & 1 & 0 & 1.967227 & 2.400559 & .900242 \\
\hline 65 & 6 & 0 & 4.987088 & 2.119608 & 2.445757 \\
\hline 66 & 1 & 0 & 6.100259 & .283438 & 2.653685 \\
\hline 67 & 1 & 0 & 3.664930 & 3.782591 & 2.077218 \\
\hline 68 & 1 & 0 & 5.735537 & 2.723356 & 2.952674 \\
\hline
\end{tabular}

Electron donation between fragments $(<0.001$ e for any omitted MO)

\begin{tabular}{|c|c|c|c|c|c|}
\hline & & & & $1->2$ & $2->1$ \\
\hline $\mathrm{HOMO}-$ & -128 & (\# & 6) & 0.002 & 0.000 \\
\hline HOMO- & -118 & (\# & 16) & 0.002 & 0.000 \\
\hline HOMO & -92 & (\# & 42) & 0.000 & 0.001 \\
\hline HOMO & -91 & (\# & 43) & -0.001 & 0.002 \\
\hline HOMO & -90 & (\# & 44) & 0.000 & 0.002 \\
\hline HOMO & -88 & (\# & 46) & 0.003 & 0.000 \\
\hline HOMO & -83 & (\# & 51) & 0.019 & 0.000 \\
\hline HOMO & -81 & (\# & 53) & 0.012 & 0.000 \\
\hline HOMO & -72 & (\# & 62) & 0.024 & 0.000 \\
\hline HOMO & -70 & (\# & 64) & 0.002 & 0.000 \\
\hline HOMO & -64 & (\# & $70)$ & 0.002 & 0.000 \\
\hline HOMO & -60 & (\# & $74)$ & 0.004 & 0.000 \\
\hline HOMO & -57 & (\# & 77) & 0.006 & 0.000 \\
\hline HOMO & -52 & (\# & 82) & 0.004 & 0.000 \\
\hline HOMO & -49 & (\# & $85)$ & 0.001 & 0.000 \\
\hline HOMO & -48 & (\# & 86) & 0.002 & 0.000 \\
\hline HOMO & -47 & (\# & 87) & 0.004 & 0.001 \\
\hline
\end{tabular}




\begin{tabular}{|c|c|c|c|c|}
\hline HOMO & -46 & $\left(\begin{array}{ll}\# & 88\end{array}\right)$ & 0.002 & 0.000 \\
\hline HOMO & -38 & $\left(\begin{array}{ll}\# & 96\end{array}\right)$ & 0.012 & 0.001 \\
\hline HOMO & -37 & (l \# 97 $)$ & 0.002 & 0.000 \\
\hline HOMO & -31 & (\#103) & 0.000 & 0.002 \\
\hline HOMO & -29 & (\#105) & 0.004 & 0.000 \\
\hline HOMO & -23 & (\#111) & 0.003 & 0.001 \\
\hline HOMO & -22 & (\#112) & 0.002 & 0.000 \\
\hline HOMO & -21 & (\#113) & 0.001 & 0.002 \\
\hline HOMO & -20 & (\#114) & 0.001 & 0.000 \\
\hline HOMO & -19 & (\#115) & 0.011 & 0.001 \\
\hline HOMO & -17 & (\#117) & 0.002 & 0.025 \\
\hline HOMO & -14 & (\#120) & 0.000 & 0.006 \\
\hline HOMO & -13 & (\#121) & 0.036 & 0.006 \\
\hline HOMO & -12 & (\#122) & 0.000 & 0.006 \\
\hline HOMO & -11 & (\#123) & 0.001 & 0.003 \\
\hline HOMO & -10 & (\#124) & 0.001 & 0.011 \\
\hline HOMO & -8 & (\#126) & 0.001 & 0.002 \\
\hline HOMO & -7 & (\#127) & 0.001 & 0.006 \\
\hline HOMO & -6 & (\#128) & 0.006 & 0.000 \\
\hline HOMO & -4 & (\#130) & 0.001 & 0.002 \\
\hline $\mathrm{HOMO}$ & -3 & (\#131) & 0.004 & 0.002 \\
\hline HOMO & -2 & (\#132) & 0.050 & 0.000 \\
\hline $\mathrm{HOMO}$ & -1 & (\#133) & 0.015 & 0.007 \\
\hline HOMO & 0 & (\#134) & 0.003 & 0.116 \\
\hline
\end{tabular}

Repulsion and residue (Delta) terms between fragments

\begin{tabular}{|c|c|c|c|c|}
\hline--- & ALPHA & $-\mathrm{SPIN}$ & $\begin{array}{c}\text { ORBITALS } \\
1<->2\end{array}$ & $\begin{array}{l}--- \\
\text { Delta }\end{array}$ \\
\hline $\mathrm{HOMO}-$ & -128 & (\# & 0.000 & 0.000 \\
\hline HOMO- & -118 & $\left(\begin{array}{l}\# \text { 16) } \\
\text { (1) }\end{array}\right.$ & 0.000 & 0.000 \\
\hline HOMO & -92 & 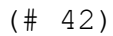 & 0.002 & 0.000 \\
\hline HOMO & -91 & 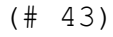 & 0.007 & 0.000 \\
\hline HOMO & -90 & (\# 44) & 0.000 & 0.000 \\
\hline HOMO & -88 & 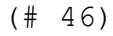 & 0.000 & 0.000 \\
\hline HOMO & -83 & (\# 51) & 0.003 & 0.000 \\
\hline HOMO & -81 & $\left(\begin{array}{l}\# \text { 53) } \\
\text { (3) }\end{array}\right.$ & 0.002 & 0.000 \\
\hline HOMO & -72 & $\left(\begin{array}{ll}\# & 62\end{array}\right)$ & 0.009 & 0.000 \\
\hline HOMO & -70 & (\# 64) & 0.002 & 0.000 \\
\hline HOMO & -64 & $\left(\begin{array}{l}\# \text { 7 } 70) \\
\text { (1) }\end{array}\right.$ & 0.012 & 0.000 \\
\hline HOMO & -60 & $\left(\begin{array}{l}\# \text { 74) } \\
\text { (3) }\end{array}\right.$ & 0.006 & 0.000 \\
\hline HOMO & -57 & $\left(\begin{array}{l}\# \text { 7 7 }\end{array}\right)$ & 0.004 & 0.000 \\
\hline HOMO & -52 & $\left(\begin{array}{l}\# \text { 82) } \\
\text { (3) }\end{array}\right.$ & 0.009 & 0.000 \\
\hline HOMO & -49 & $\left(\begin{array}{l}\# \text { 85) } \\
\text { (n) }\end{array}\right.$ & 0.005 & 0.000 \\
\hline HOMO & -48 & $\left(\begin{array}{l}\# \text { 86) } \\
\text { (n) }\end{array}\right.$ & 0.005 & 0.000 \\
\hline HOMO & -47 & $\left(\begin{array}{l}\# \text { 87) } \\
\text { (3) }\end{array}\right.$ & 0.008 & 0.000 \\
\hline HOMO & -46 & 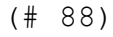 & 0.003 & 0.000 \\
\hline HOMO & -38 & $\left(\begin{array}{l}\# \text { 96) } \\
\text { (n) }\end{array}\right.$ & 0.023 & -0.001 \\
\hline HOMO & -37 & (\# 97) & 0.001 & 0.000 \\
\hline HOMO & -31 & (\#103) & -0.002 & 0.000 \\
\hline HOMO & -29 & (\#105) & 0.010 & 0.000 \\
\hline HOMO & -23 & (\#111) & 0.008 & 0.000 \\
\hline HOMO & -22 & (\#112) & 0.004 & 0.000 \\
\hline HOMO & -21 & (\#113) & 0.001 & 0.000 \\
\hline HOMO & -20 & (\#114) & 0.002 & 0.000 \\
\hline HOMO & -19 & (\#115) & 0.018 & -0.001 \\
\hline HOMO & -17 & (\#117) & -0.010 & -0.001 \\
\hline HOMO & -14 & (\#120) & -0.002 & 0.000 \\
\hline HOMO & -13 & (\#121) & -0.061 & -0.002 \\
\hline HOMO & -12 & (\#122) & -0.011 & 0.000 \\
\hline $\mathrm{HOMO}$ & -11 & (\#123) & -0.014 & 0.000 \\
\hline HOMO & -10 & (\#124) & -0.010 & 0.000 \\
\hline HOMO & -8 & $(\# 126)$ & -0.017 & 0.000 \\
\hline
\end{tabular}




$\begin{array}{lrlll}\text { HOMO } & -7 & (\# 127) & -0.002 & 0.000 \\ \text { HOMO } & -6 & (\# 128) & -0.018 & 0.000 \\ \text { HOMO } & -4 & (\# 130) & -0.012 & 0.000 \\ \text { HOMO } & -3 & (\# 131) & -0.012 & 0.000 \\ \text { HOMO } & -2 & (\# 132) & -0.085 & 0.001 \\ \text { HOMO } & -1 & (\# 133) & -0.021 & 0.001 \\ \text { HOMO } & 0 & (\# 134) & -0.031 & 0.002 \\ ----------------------- \\ \text { Total over OMOs } & -0.171 & -0.004\end{array}$

ONIOM optimization and CDA calculations 2er.

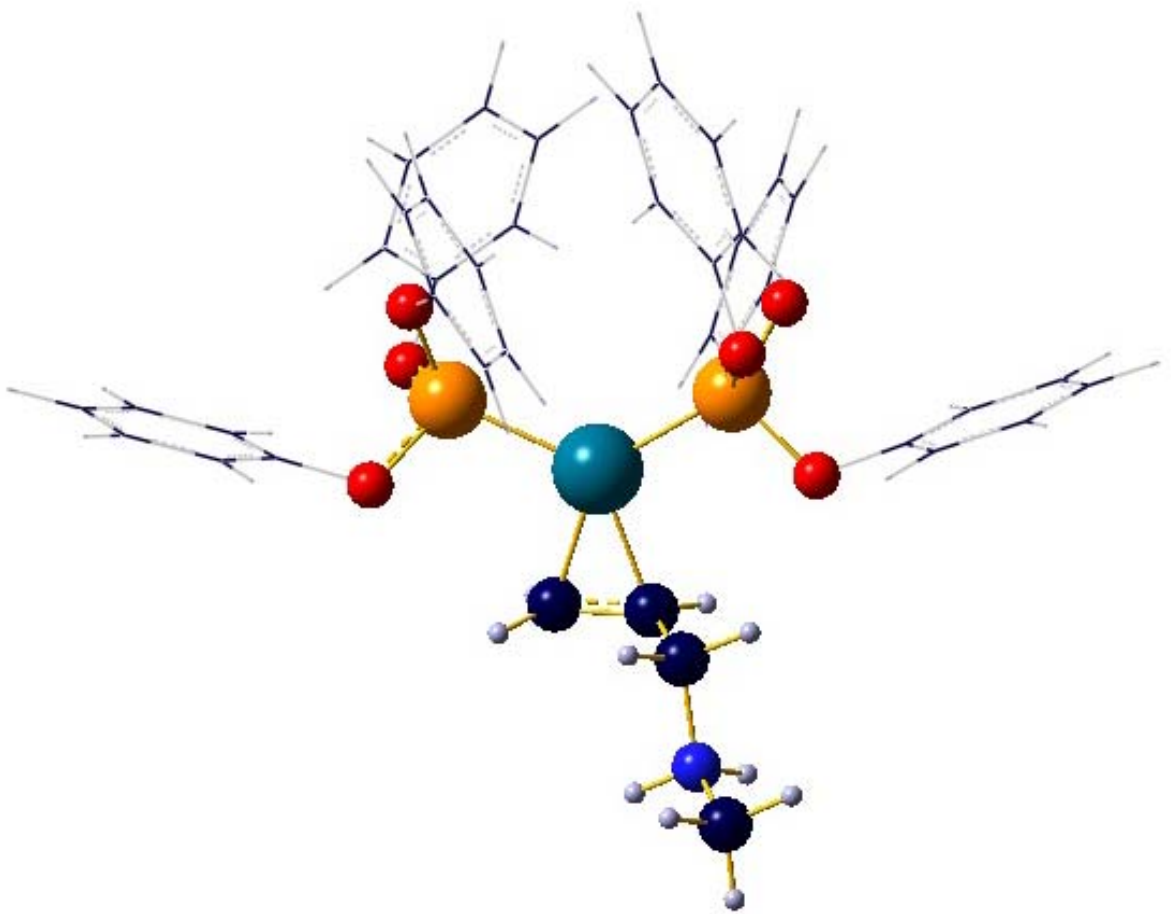

Standard orientation:

\begin{tabular}{|c|c|c|c|c|c|}
\hline \multirow{2}{*}{$\begin{array}{l}\text { Center } \\
\text { Number }\end{array}$} & \multirow{2}{*}{$\begin{array}{l}\text { Atomic } \\
\text { Number }\end{array}$} & \multirow{2}{*}{$\begin{array}{c}\text { Atomic } \\
\text { Type }\end{array}$} & \multicolumn{3}{|c|}{ Coordinates (Angstroms) } \\
\hline & & & $\mathrm{X}$ & $\mathrm{Y}$ & Z \\
\hline 1 & 6 & 10061003 & -1.561209 & 3.550629 & -1.644454 \\
\hline 2 & 6 & 10061003 & 0.573982 & 2.465144 & -2.472693 \\
\hline 3 & 6 & 10061003 & -0.834994 & 2.476198 & -2.382644 \\
\hline 4 & 1 & 10011000 & -2.539394 & 3.228593 & -1.279483 \\
\hline 5 & 1 & 10011000 & 1.064682 & 1.953342 & -3.297839 \\
\hline 6 & 1 & 10011000 & -1.426354 & 1.940714 & -3.126801 \\
\hline 7 & 1 & 10011000 & -0.977112 & 3.965049 & -0.818100 \\
\hline 8 & 1 & 10011000 & 1.179126 & 3.227268 & -1.981514 \\
\hline 9 & 7 & 10071003 & -1.833200 & 4.746886 & -2.580716 \\
\hline 10 & 1 & 10011000 & -2.375941 & 4.402274 & -3.379036 \\
\hline 11 & 1 & 10011000 & -0.931305 & 5.038849 & -2.972110 \\
\hline 12 & 6 & 10061003 & -2.530124 & 5.905306 & -1.949991 \\
\hline 13 & 1 & 10011000 & -2.683741 & 6.688976 & -2.694445 \\
\hline 14 & 1 & 10011000 & -3.489948 & 5.563279 & -1.559947 \\
\hline 15 & 1 & 10011000 & -1.911745 & 6.280202 & -1.133049 \\
\hline 16 & 46 & 10461004 & -0.029979 & 1.034968 & -0.995167 \\
\hline 17 & 15 & 10151004 & 2.133983 & 0.448568 & -0.390603 \\
\hline 18 & 15 & 10151004 & -1.954441 & 0.145027 & -0.043908 \\
\hline 19 & 8 & 10081000 & -3.221339 & 1.014190 & -0.670322 \\
\hline 20 & 8 & 10081000 & -2.406069 & -1.427223 & -0.283089 \\
\hline 21 & 8 & 10081000 & -2.366526 & 0.302796 & 1.543295 \\
\hline 22 & 8 & 10081000 & 2.577777 & -0.241730 & 1.048368 \\
\hline 23 & 8 & 10081000 & 3.030319 & 1.822438 & -0.485483 \\
\hline
\end{tabular}




\begin{tabular}{|c|c|c|c|c|c|}
\hline 24 & 8 & 10081000 & 3.012201 & -0.511891 & -1.390771 \\
\hline 25 & 6 & 10061000 & -4.478615 & 0.578006 & -0.316999 \\
\hline 26 & 6 & 10061000 & -5.126589 & -0.403903 & -1.081313 \\
\hline 27 & 6 & 10061000 & -5.153274 & 1.177734 & 0.756021 \\
\hline 28 & 6 & 10061000 & -6.418165 & -0.818338 & -0.741911 \\
\hline 29 & 1 & 10011000 & -4.627420 & -0.846365 & -1.934070 \\
\hline 30 & 6 & 10061000 & -6.444640 & 0.761404 & 1.094093 \\
\hline 31 & 1 & 10011000 & -4.672856 & 1.959331 & 1.330859 \\
\hline 32 & 6 & 10061000 & -7.075231 & -0.239198 & 0.348131 \\
\hline 33 & 1 & 10011000 & -6.911332 & -1.584280 & -1.326283 \\
\hline 34 & 1 & 10011000 & -6.957921 & 1.216989 & 1.930966 \\
\hline 35 & 1 & 10011000 & -8.075383 & -0.559756 & 0.609516 \\
\hline 36 & 6 & 10061000 & -1.954797 & -0.589191 & 2.515539 \\
\hline 37 & 6 & 10061000 & -2.551146 & -0.481385 & 3.779582 \\
\hline 38 & 6 & 10061000 & -0.954634 & -1.567169 & 2.320222 \\
\hline 39 & 6 & 10061000 & -2.178037 & -1.335662 & 4.821359 \\
\hline 40 & 1 & 10011000 & -3.310367 & 0.270590 & 3.954716 \\
\hline 41 & 6 & 10061000 & -0.588794 & -2.424459 & 3.361746 \\
\hline 42 & 1 & 10011000 & -0.438268 & -1.662152 & 1.380997 \\
\hline 43 & 6 & 10061000 & -1.199457 & -2.308978 & 4.611617 \\
\hline 44 & 1 & 10011000 & -2.647084 & -1.241335 & 5.792128 \\
\hline 45 & 1 & 10011000 & 0.181073 & -3.168615 & 3.203170 \\
\hline 46 & 1 & 10011000 & -0.908600 & -2.967870 & 5.419356 \\
\hline 47 & 6 & 10061000 & -1.875246 & -2.152787 & -1.332305 \\
\hline 48 & 6 & 10061000 & -2.036978 & -3.544638 & -1.297347 \\
\hline 49 & 6 & 10061000 & -1.218638 & -1.573798 & -2.443957 \\
\hline 50 & 6 & 10061000 & -1.533987 & -4.348954 & -2.324171 \\
\hline 51 & 1 & 10011000 & -2.548352 & -4.006800 & -0.462287 \\
\hline 52 & 6 & 10061000 & -0.711011 & -2.382999 & -3.464814 \\
\hline 53 & 1 & 10011000 & -1.101189 & -0.505669 & -2.540453 \\
\hline 54 & 6 & 10061000 & -0.865491 & -3.769066 & -3.403866 \\
\hline 55 & 1 & 10011000 & -1.657001 & -5.423233 & -2.278801 \\
\hline 56 & 1 & 10011000 & -0.200641 & -1.933552 & -4.306845 \\
\hline 57 & 1 & 10011000 & -0.470419 & -4.393283 & -4.194749 \\
\hline 58 & 6 & 10061000 & 1.979218 & 0.152022 & 2.230864 \\
\hline 59 & 6 & 10061000 & 2.336806 & -0.546625 & 3.392318 \\
\hline 60 & 6 & 10061000 & 1.057912 & 1.222486 & 2.339566 \\
\hline 61 & 6 & 10061000 & 1.784723 & -0.206629 & 4.630575 \\
\hline 62 & 1 & 10011000 & 3.043758 & -1.364533 & 3.332809 \\
\hline 63 & 6 & 10061000 & 0.509663 & 1.558310 & 3.581167 \\
\hline 64 & 1 & 10011000 & 0.753383 & 1.804081 & 1.481084 \\
\hline 65 & 6 & 10061000 & 0.869500 & 0.842891 & 4.724417 \\
\hline 66 & 1 & 10011000 & 2.063214 & -0.761389 & 5.517098 \\
\hline 67 & 1 & 10011000 & -0.200441 & 2.371726 & 3.655546 \\
\hline 68 & 1 & 10011000 & 0.437903 & 1.100885 & 5.682720 \\
\hline 69 & 6 & 10061000 & 2.845054 & -1.883234 & -1.418982 \\
\hline 70 & 6 & 10061000 & 3.816475 & -2.638488 & -2.090470 \\
\hline 71 & 6 & 10061000 & 1.745621 & -2.554173 & -0.840165 \\
\hline 72 & 6 & 10061000 & 3.713151 & -4.030888 & -2.164778 \\
\hline 73 & 1 & 10011000 & 4.660485 & -2.142180 & -2.553052 \\
\hline 74 & 6 & 10061000 & 1.651646 & -3.947135 & -0.905522 \\
\hline 75 & 1 & 10011000 & 0.953567 & -2.011329 & -0.353246 \\
\hline 76 & 6 & 10061000 & 2.633182 & -4.685241 & -1.568900 \\
\hline 77 & 1 & 10011000 & 4.472105 & -4.602437 & -2.683180 \\
\hline 78 & 1 & 10011000 & 0.811170 & -4.453772 & -0.449396 \\
\hline 79 & 1 & 10011000 & 2.554449 & -5.763183 & -1.625178 \\
\hline 80 & 6 & 10061000 & 4.348301 & 1.707802 & -0.104203 \\
\hline 81 & 6 & 10061000 & 5.331126 & 1.363905 & -1.044758 \\
\hline 82 & 6 & 10061000 & 4.727055 & 2.021118 & 1.209306 \\
\hline 83 & 6 & 10061000 & 6.672965 & 1.282260 & -0.659908 \\
\hline 84 & 1 & 10011000 & 5.054347 & 1.157586 & -2.070794 \\
\hline 85 & 6 & 10061000 & 6.069300 & 1.938476 & 1.592019 \\
\hline 86 & 1 & 10011000 & 3.980769 & 2.323426 & 1.932728 \\
\hline 87 & 6 & 10061000 & 7.041359 & 1.564673 & 0.659052 \\
\hline 88 & 1 & 10011000 & 7.427473 & 1.005283 & -1.384754 \\
\hline 89 & 1 & 10011000 & 6.356486 & 2.168877 & 2.609777 \\
\hline 90 & 1 & 10011000 & 8.080361 & 1.502578 & 0.955689 \\
\hline
\end{tabular}


Electron donation between fragments $(<0.001$ e for any omitted MO)

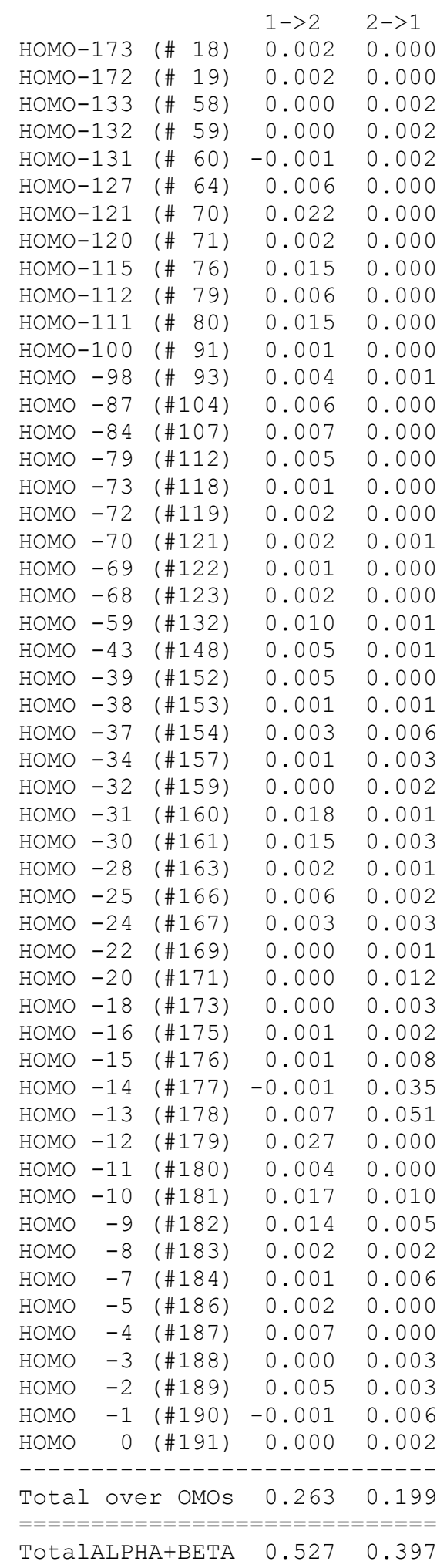

Repulsion and residue (Delta) terms between fragments

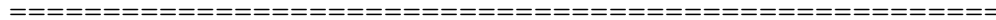

$\begin{array}{cccc}--- \text { ALPHA-SPIN } & \text { ORBITALS } & --- \\ & & 1<->2 & \text { Delta } \\ \text { HOMO-173 (\# 18) } & 0.000 & 0.000 \\ \text { HOMO-172 (\# 19) } & 0.000 & 0.000\end{array}$




\begin{tabular}{|c|c|c|c|c|}
\hline \multicolumn{2}{|c|}{$\mathrm{HOMO}-133$} & $\left(\begin{array}{l}\# \text { 58) } \\
\text { (3) }\end{array}\right.$ & 0.002 & 0.000 \\
\hline \multicolumn{2}{|c|}{ HOMO-132 } & (\# 59) & 0.000 & 0.000 \\
\hline \multicolumn{2}{|c|}{ HOMO-131 } & $\left(\begin{array}{l}\# \text { 60) } \\
\text { (1) }\end{array}\right.$ & 0.005 & .000 \\
\hline \multicolumn{2}{|c|}{ HOMO-127 } & $\left(\begin{array}{l}\# \text { 64) } \\
\text { (1) }\end{array}\right.$ & 0.000 & .000 \\
\hline \multicolumn{2}{|c|}{ HOMO-121 } & $\left(\begin{array}{ll}\# & 70\end{array}\right)$ & 0.006 & .000 \\
\hline \multicolumn{2}{|c|}{ HOMO-120 } & $\left(\begin{array}{l}\# \text { 71) } \\
\text { (1) }\end{array}\right.$ & -0.001 & .000 \\
\hline \multicolumn{2}{|c|}{ HOMO-115 } & $\left(\begin{array}{ll}\# & 76\end{array}\right)$ & 0.003 & .00 \\
\hline \multicolumn{2}{|c|}{ HOMO-112 } & $\left(\begin{array}{ll}\# & 79\end{array}\right)$ & 0.004 & 0 \\
\hline \multicolumn{2}{|c|}{ HOMO-111 } & $\left(\begin{array}{l}\# \text { 80) } \\
\text { (1) }\end{array}\right.$ & 0.004 & 0 \\
\hline \multicolumn{2}{|c|}{ HOMO-100 } & $\left(\begin{array}{l}\# \text { 91) } \\
\text { (1) }\end{array}\right.$ & 0.001 & \\
\hline HOMO & -98 & $\left(\begin{array}{ll}\# \text { 93) }\end{array}\right.$ & 0.019 & 0 \\
\hline HOMO & -87 & (\#104) & 0.010 & 0 \\
\hline HOMO & -84 & (\#107) & 0.006 & 0 \\
\hline HOMO & -79 & (\#112) & 0.020 & 0.000 \\
\hline HOMO & -73 & (\#118) & 0.002 & .000 \\
\hline HOMO & -72 & (\#119) & 0.007 & 0.000 \\
\hline HOMO & -70 & (\#121) & -0.001 & 0.000 \\
\hline HOMO & -69 & (\#122) & 0.009 & 0.000 \\
\hline HOMO & -68 & (\#123) & 0.007 & 0.000 \\
\hline HOMO & -59 & (\#132) & 0.014 & -0.001 \\
\hline HOMO & -43 & (\#148) & 0.006 & 0.000 \\
\hline HOMO & -39 & (\#152) & 0.014 & 0.000 \\
\hline HOMO & -38 & (\#153) & 0.000 & 0.000 \\
\hline HOMO & -37 & (\#154) & 0.001 & -0.001 \\
\hline HOMO & -34 & (\#157) & -0.001 & 0.000 \\
\hline HOMO & -32 & (\#159) & -0.002 & 0.000 \\
\hline HOMO & -31 & (\#160) & 0.001 & -0.001 \\
\hline HOMO & -30 & (\#161) & -0.008 & -0.001 \\
\hline HOMO & -28 & (\#163) & -0.001 & 0.00 \\
\hline HOMO & -25 & (\#166) & -0.013 & -0.00 \\
\hline HOMO & -24 & (\#167) & -0.009 & 0.00 \\
\hline HOMO & -22 & (\#169) & -0.001 & 0.00 \\
\hline HOMO & -20 & (\#171) & -0.006 & 0.00 \\
\hline HOMO & -18 & (\#173) & -0.002 & 0.00 \\
\hline HOMO & -16 & (\#175) & -0.016 & 0.00 \\
\hline HOMO & -15 & (\#176) & -0.042 & 0.00 \\
\hline HOMO & -14 & (\#177) & -0.017 & 0.002 \\
\hline HOMO & -13 & (\#178) & -0.037 & 0.003 \\
\hline HOMO & -12 & (\#179) & -0.042 & 0.000 \\
\hline HOMO & -11 & (\#180) & -0.007 & 0.000 \\
\hline HOMO & -10 & (\#181) & -0.032 & 0.001 \\
\hline HOMO & -9 & (\#182) & -0.035 & 0.000 \\
\hline HOMO & -8 & (\#183) & -0.004 & 0.000 \\
\hline HOMO & -7 & (\#184) & -0.005 & 0.000 \\
\hline HOMO & -5 & (\#186) & -0.003 & 0.000 \\
\hline HOMO & -4 & (\#187) & -0.009 & 0.000 \\
\hline HOMO & -3 & (\#188) & -0.002 & 0.000 \\
\hline HOMO & -2 & (\#189) & -0.010 & 0.00 \\
\hline HOMO & -1 & (\#190) & -0.002 & 0.00 \\
\hline $\mathrm{HOMO}$ & 0 & (\#191) & -0.003 & 0.000 \\
\hline
\end{tabular}

